\title{
Ballistic Electron Transport in Graphene Nanodevices and Billiards
}

\author{
Dissertation \\ (Cumulative Thesis) \\ for the award of the degree
}

\section{Doctor rerum naturalium}

of the Georg-August-Universität Göttingen

within the doctoral program

Physics of Biological and Complex Systems

of the Georg-August University School of Science

submitted by

George Datseris

from Athens, Greece

Göttingen 2019 


\section{Thesis advisory committee}

Prof. Dr. Theo Geisel

Department of Nonlinear Dynamics

Max Planck Institute for Dynamics and Self-Organization

Prof. Dr. Stephan Herminghaus

Department of Dynamics of Complex Fluids

Max Planck Institute for Dynamics and Self-Organization

Dr. Michael Wilczek

Turbulence, Complex Flows \& Active Matter

Max Planck Institute for Dynamics and Self-Organization

\section{Examination board}

Prof. Dr. Theo Geisel (Reviewer)

Department of Nonlinear Dynamics

Max Planck Institute for Dynamics and Self-Organization

Prof. Dr. Stephan Herminghaus (Second Reviewer)

Department of Dynamics of Complex Fluids

Max Planck Institute for Dynamics and Self-Organization

Dr. Michael Wilczek

Turbulence, Complex Flows \& Active Matter

Max Planck Institute for Dynamics and Self-Organization

Prof. Dr. Ulrich Parlitz

Biomedical Physics

Max Planck Institute for Dynamics and Self-Organization

Prof. Dr. Stefan Kehrein

Condensed Matter Theory, Physics Department

Georg-August-Universität Göttingen

Prof. Dr. Jörg Enderlein

Biophysics / Complex Systems, Physics Department

Georg-August-Universität Göttingen

Date of the oral examination: September $13^{\text {th }}, 2019$ 


\section{Contents}

$\begin{array}{lr}\text { Abstract } & 3\end{array}$

1 Introduction $\quad \mathbf{5}$

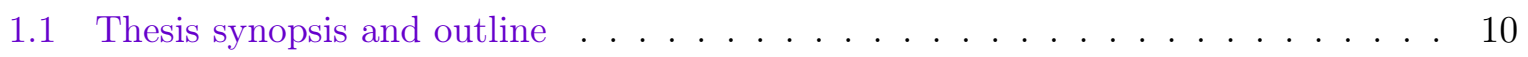

2 Fundamental Concepts $\quad 13$

2.1 Nonlinear dynamics of antidot superlattices . . . . . . . . . . . . . . . . 13

2.2 Lyapunov exponents in billiards . . . . . . . . . . . . . . . . . . . . . . . . 20

2.3 Fundamental properties of graphene . . . . . . . . . . . . . . . . . . . . 22

2.4 Why quantum? . . . . . . . . . . . . . . . . . . . . . . . . 31

2.5 Transport simulations and scattering wavefunctions . . . . . . . . . . . . . 33

2.6 Quantum mechanics and the phase space . . . . . . . . . . . . . . . . . . 34

$31^{\text {st }}$ paper: Robustness of ballistic transport in antidot superlattices 38

3.1 Hall resistance . . . . . . . . . . . . . . . . . . . . . . . . . . 48

4 Software to simulate dynamical billiards $\quad 51$

4.1 Design ................................. 51

4.2 Usage in my projects . . . . . . . . . . . . . . . . . . . . . . . 53

4.3 Unique features . . . . . . . . . . . . . . . . . . . . . 54

$5 \quad 2^{\text {nd }}$ paper: Estimating Lyapunov exponents in billiards 55

$6 \quad 3^{\text {rd }}$ paper: Phase space analysis of quantum transport in graphene $\quad 69$

7 Husimi functions and Klein tunneling in magnetic fields 81

7.1 Husimi function in magnetic fields . . . . . . . . . . . . . . . . . . . . . . . . . . 81

7.2 Application to a graphene nanodevice . . . . . . . . . . . . . . . . . . . . 84

7.3 Klein tunneling in a pn-junction with magnetic field . . . . . . . . . . . . . . . 85

8 Summary \& Outlook $\quad 89$

8.1 Summary of results . . . . . . . . . . . . . . . . . . . . . . 89

8.2 Outlook .............................. . . . 90

$\begin{array}{lr}\text { Acknowledgments } & 92\end{array}$

$\begin{array}{lr}\text { Bibliography } & 92\end{array}$

$\begin{array}{lr}\text { Curriculum Vitae } & 101\end{array}$ 


\section{Abstract}

Nanodevices are objects with broad relevance for today's society, which bases its economy, operation, and communication onto digital resources. In ballistic nanodevices, disorder has either negligible or minor impact on electron transport. These ballistic devices are then typical components in computers and digital storage units. This cumulative thesis theoretically studies ballistic electron transport in graphene-based nanodevices and billiards. The thesis is divided into two main parts: the first part uses classical dynamical systems theory, while the second part applies semiclassical approaches to quantum transport.

The first part of the thesis is motivated by recent graphene experiments, that show puzzling measurements of the resistance versus the magnetic field strength in an antidot superlattice nanodevice, a two-dimensional periodic array of repellers patterned on top of a crystalline lattice (in this case, graphene). The resistance of an antidot superlattice shows pronounced peaks when the strength of an applied perpendicular magnetic field is varied. The peaks, called commensurability peaks, occur when the cyclotron radius becomes commensurable with the geometry of the superlattice. This effect was been studied before, and it was shown that classical theory and nonlinear dynamics are best suited to understand it. What is puzzling is that existing theories cannot account for the observed peaks, when the electron mean free time (average time between disorder-induced scatterings) in the device is as small as in the recent experiments. By finding the inherent timescales of the dynamical system that represents the nanodevice, we provide the necessary theoretical background to explain the experiments and understand how it is possible to observe the effect. In the process of doing so, we also resolve a long-standing controversy on the origin of the effect and its connection with the various parts of the electron dynamics in an antidot superlattice. In addition, analytic connections are made between characteristic timescales in dynamical systems and the volume of sets in the phase space, and the impact of volume conservation of Hamiltonian mechanics is demonstrated.

These analytic connections are further taken advantage of in dynamical billiards, systems typically used to model real nanodevices. There, we show that generically in billiards the Lyapunov exponent, a number quantifying the chaotic dynamics of a system, has a leading contribution inversely proportional to the chaotic phase space volume. This finding has theoretical value in itself, but it also allows one to readily connect the Lyapunov exponent with the parameters of the billiard, which has implications for using billiards to model real physical systems.

The results of the first part of the thesis have implications in understanding transport in many models following classical dynamics, because of the very simple principles used in deriving analytic relations between transport timescales and phase space volumes. Also, our results suggest that if a relevant dynamic timescale is larger than the mean free time, then a reasonable expectation is that the dynamic feature will not be observable at all in experiments. Thus, knowing exactly which is the important timescale is crucial even before starting an experimental measurement.

The second part of this thesis is motivated by quantum processes specific to graphene, as well as questions regarding quantum-classical correspondence. Specifically, electrons in graphene 
undergo a special tunneling process called Klein tunneling. There, a particle incident on a potential barrier can penetrate the barrier with perfect transmission (100\% probability), provided that the incident particle reaches the barrier with zero angle of incidence (i.e. normal incidence). Oddly, this is true irrespectively of the characteristics of the potential. From its definition, Klein tunneling is a process that depends on the angle of incidence of the particle, a concept that has classical intuition. Furthermore, even though possible to define in a trivial plane wave eigenfunction, defining the angle of incidence in a complicated quantum transport setting is not at all trivial. To be able to define concepts such as the angle of incidence, and obtain classical intuition about them in a quantum transport numerical experiment, in this thesis we use the Husimi function, in both the absence and presence of magnetic fields.

The Husimi function is a tool that can transform a quantum wavefunction into a probability distribution over the phase space of position and momentum, which is useful for defining concepts such as the angle of incidence. The Husimi function also helps to quantitatively define more complicated concepts, like intervalley scattering, and even in devices with non-trivial geometries. We also show how, through the Husimi function, one can recover the transmission probabilities of electronic wavefunctions being transmitted through a nanodevice, by interpreting the marginal distributions of the Husimi function as weighting probabilities of a transmission formula. We then quantitatively measure the effect that pn-junctions and geometric scattering have on intervalley scattering. Surprisingly, we show that pn-junctions can intervalley-scatter only one of the two valleys of graphene. In the literature, it has been qualitatively suggested that the armchair termination of graphene is the strongest geometric intervalley scatterer. Here, we prove this to be true using quantitative measurements.

Lastly, for the first time in the literature, we extend the Husimi function for electrons moving in magnetic fields while respecting fundamental energy considerations. We further use this new tool to study Klein tunneling in magnetic fields. Our results demonstrate that Klein tunneling in magnetic fields is not well understood yet since our numeric study does not align with the existing theory on the effect. The entire second part of this thesis showcases that the Husimi function is very likely to help unravel transport phenomena in nanodevices. Thus, it could be of general interest for condensed matter theory, currently a field that does not utilize the Husimi function at all. 


\section{1 | Introduction}

The $21^{\text {st }}$ century has been dubbed by many the Information Age or Digital Age [1], due to the rapid shift from the previous industry-based economy to a society that bases its economy, operation and communication onto digital resources. It is not an exaggeration to say that in the present day it would be impossible for society to operate without digital information and computers to handle it. As time progresses, digital computation takes an ever-increasing role in society and economy. For example, the new concept of "cryptocurrencies" [2,3] is an economic model based entirely on digital assets which are created on the basis of computation. Scientific progress is also becoming increasingly more reliant on both digital information and digital computation. Scientific models that aim to understand and predict the most fundamental aspects of the world, like the weather $[4,5]$ or the quantum realm [6], require tremendous amount of both computing power as well as digital storage to operate properly.

But what enables all of this? What is the key that opened the door to this new Digital Age? By large part, it is nanodevices and our improved understanding and fabrication of them. For example, the transistor, one of the biggest discoveries of humankind [7], is a simple nanodevice that can amplify or switch on and off electric current. Understanding its underlying physical principles, how to use it in circuits to great effect, and how to make increasingly more efficient transistors was exactly what allowed the fabrication of the early computers and ushered humanity into the digital age [7].

The progress that lead to the Digital Age did not happen instantly, nor by chance. Advances in engineering were important to fabricate better, more efficient, and smaller devices. Advances in experimental physics were necessary to invent new materials as nanodevice basis, as well as to measure complex phenomena. And finally, advances in theoretical physics were necessary to understand the new materials, explain the measured complex phenomena, and demonstrate how the physical principles underlying the devices may be used in application scenarios.

\section{Nanodevices \& graphene}

Making a nanodevice is an extremely difficult task. By definition, to make a nanodevice one must be able to fabricate structures with sufficient accuracy on the order of 10 to 100 nanometers [8]. The reason that this is so hard is because the typical distance between the atoms that make up the crystalline lattice of a metal or alloy is about 0.1 to 0.3 nanometers [9]. Therefore, making a nanodevice amounts to having accurate control to e.g. deposit or remove around 100 to 1000 atoms. Although it is possible to achieve such a control, it is nearly impossible to achieve it in perfection. As a result, fabricated nanodevices contain several structural disorders, defects, impurities and other factors, which for our purposes are noise-inducing sources in the device. The electrons that are transported through the device interact with these factors, typically in a stochastic manner. More often than not, these interactions are inhibiting the propagation 
and control of electric current. Thankfully, experimental advances in fabrication techniques are constantly happening. This gives more and more control over the fabrication process and reduces the noise, resulting in "cleaner" devices.

Experimental advances also lead to the discovery of new materials. Standard materials for fabricating nanodevices and transistors were, and still are, gallium arsenide (GaAs) [10] as well as silicon [11] based semiconductors and heterostructures. In 2004 however, experimentalists were able to fabricate for the first time a new material, and in fact a new class of materials, graphene $[12,13,14,15]$. Graphene is a simple material, at least on paper: it is composed exclusively of carbon atoms and is purely two dimensional, with the carbon atoms existing in a single atomic layer, arranged in a honeycomb pattern like in Fig. 1.1. Even though a material that is only a single layer of atoms thick seems something unfamiliar with everyday life a stack of many of these layers is simply graphite (the material used in pencils). What is special about graphene, however, is not its purely two-dimensional nature, but that its structure and symmetries have brought completely new physics into condensed matter theory.

In a typical semiconductor like e.g. GaAs or silicon-based, the electrons follow what is called a "parabolic dispersion relation", which means that the energy associated with an electron scales with the square of the wavevector associated with the electron wavefunction, $E \sim \mathbf{k}^{2}$. In the classical limit where the wavevector is replaced with the momentum, this means that the electrons follow the well known classical Newtonian dynamics. In graphene the case is different. Here, the dispersion relation is not parabolic, but conic instead, $E \sim|\mathbf{k}|$. In the classical limit, this relation means that the electrons move as hyper-relativistic particles, i.e. not only do they follow relativistic equations of motion instead of Newtonian ones, but they also behave as if they have zero mass (i.e. like photons). Of course, this also applies in the quantum case, where the electrons there are labeled Dirac particles, in honor of Paul Dirac who was one of the pioneers to discuss quantum relativistic phenomena and the dispersion $E \sim|\mathbf{k}|$ in detail [16].

The basic physics of graphene lead to some remarkable properties. Graphene is a zero-gap semiconductor, meaning that it can always conduct current. It has resistivity smaller than silver at room temperature, at around $10^{-6} \Omega / \mathrm{m}$, which is the lowest known at room temperature $[15$, 17]. Its thermal conductivity exceeds that of diamond [18] while its mechanical properties establish it as one of the strongest materials ever tested [19]. These, and many more almost exotic properties concern the infinite sheet of pure graphene (for more details see [20,21] and references therein) and it is because of these properties that graphene was heralded as the substitute for silicon that will lead to new age electronics. Examples include applications in transistors, touch-screens, biomedicine [22] and energy, to name a few (see e.g. [23, 24] and references therein). However, up to the point of writing this thesis, graphene has not taken over silicon and is in fact not that widespread in real-world applications. The reason is because it is exceptionally difficult, as well as expensive, to manufacture sufficiently clean graphene and in large quantities [25]. Progress is rapid though [25], and sufficiently clean devices based on graphene are now being created by experimentalists and studied by theorists.

\section{Classical theory for ballistic nanodevices}

Clearly, the amount of disorder (and its minimization) is a crucial concept in the study of nanodevices since it can even forbid the technological application of an otherwise promising material. A qualitative description of how important is the noise level in a device rests on the concept of a ballistic device $[8,26]$. By definition, a device is called ballistic when the noise-induced scattering of the electrons is either negligible or plays a minor role in the dynamics of the device. In the ballistic scenario, the electrons inside the device move mostly according to deterministic 
Hamiltonian mechanics (either classical or quantum) and the electron motion is dictated by the device geometry as well as existing electromagnetic potentials. A quantitative way to define a device as ballistic is to look at its mean free time $\tau$. Simply put, $\tau$ quantifies on average for how much time the electrons move in a device until interactions with the noise-inducing factors significantly disrupt the electron motion. If $\tau \cdot v$ (with $v$ the average carrier velocity) is larger than the characteristic length scales of the device or of the present electromagnetic potentials, then the device is typically ballistic. Of course, even though it is a difficult task, only making a ballistic device is not enough; one also has to understand its physics, both for interpretation of experiments as well as for potential technological applications. And since ballistic realizations of complex nanodevices in e.g. graphene have started becoming a reality only recently, it is now a prime time to theoretically analyze and understand these new devices while comparing with the newly accessible experimental data.

One aspect that is highly relevant for applications, experiments, as well as theory, is understanding and controlling electronic transport, i.e. the movement of electrons (and by extension, the transport of charge i.e. electric current) in a ballistic device. To do this, many different approaches have been implemented by physicist [27, 8], for example the density functional theory [28] or tight-binding simulations [27, 29]. In this dissertation, however, the focus lies in the following two approaches: using the dynamical systems framework and phase space representation of quantum mechanics. Dynamical systems theory is an abstract mathematical formalism that can describe many different physical systems. A dynamical system is represented by a collection of variables (e.g. position and momentum of an electron, voltage and current in an electronic circuit, temperature and pressure of an air parcel, chemical concentrations, and in general any physical system) whose value changes with time. The dynamical system then is modeled by a set of coupled ordinary differential equations, or coupled discrete maps. Note that the classical Hamilton's equation of motion are also part of dynamical system theory. Dynamical systems have been successful in modeling essentially every part of the physical world, from weather [30], to fluctuation of populations [31] to the heart [32] to microbes [33] and more. Specifically within the context of nanodevices, they have been used in e.g. quantum dots [34], optical lattices [35] and antidot superlattices [36] (see also sec. 2.1.2). An example of an antidot superlattice, a nanodevice that will be discussed at length in later chapters of this dissertation, is shown in Fig. 1.1(b, c).

A subclass of classical Hamiltonian systems, that are very relevant for modeling nanodevices, are dynamical billiards [37]. In billiards the dynamic variables are always the position and momentum of a point particle (here an electron). This particle propagates freely within a domain (which often represents a nanodevice) and only upon colliding with the boundary of the domain the particle is reflected specularly, as is shown in Fig. 1.1(d), which shows the "mushroom" billiard. Billiards are relevant in theoretical studies and modeling of nanodevices and have been used extensively to model nanodevices both from a classical [38, 36, 39, 40, 41, 42, 43, 44, 45, 46] as well as quantum perspective [47, 48, 49, 50], and have also been implemented extensively specifically for graphene [51, 46, 52, 53].

Generally dynamical systems posses intrinsic characteristic timescales. These timescales arise from the inherent dynamics of the system and can have profound relevance not only for the dynamics, but also for the behavior of the real systems that the dynamical system models. For example, if we imagine the mushroom billiard of Fig. 1.1(d) to represent a nanodevice where electrons are injected from the stem bottom, and can only leave the device again from the stem bottom, then an important timescale of the system would be the average amount of time the electrons need to return to the stem bottom. One more example of an important timescale is the average travel time between antidots in an antidot superlattice (Fig. 1.1(b,c)), which is discussed in the first paper of this dissertation, in chapter 3. Finding these characteristic timescales, and 
understanding how they depend on the parameters of the nanodevice is crucial in the context of ballistic transport. An important question is how these inherent timescales compare with the mean free time $\tau$. One can imagine that if $\tau$ is shorter than the inherent timescales, the associated part of the dynamics could be inaccessible by experiments in the real system.

But besides system-specific timescales, there is one more timescale that is of general interest for dynamical systems: the Lyapunov exponent $[54,55,56,57]$. Most dynamical systems have nonlinear equations of motion [58], which quite often results in chaotic evolution of the dynamical variables (e.g. chaotic motion of electrons inside nanodevices). It is broadly defined that the dynamics of a system are chaotic if initially close trajectories separate exponentially fast as they are evolved with time [59]. Chaos also leads to analytically intractable evolution of the dynamic variables in time $[58,60,59]$. Because of this chaotic property, it is impossible to exactly predict the long term behavior of a dynamical system. This happens because there is always finite precision in determining the initial condition of the system, from which the evolution in time will start. This initial inaccuracy grows with time in chaotic systems, until the inaccuracy actually covers the entire phase space, meaning that the system could be in any of the all possible states it is allowed to be. The Lyapunov exponent establishes a timescale $t_{\lambda}$ which, in order to increase the time of an accurate prediction linearly (in multiples of $t_{\lambda}$ ), one has to increase the initial accuracy geometrically [61]. In a sense this quantifies the "predictability horizon" of a chaotic system, since predictions above some multiples of this timescale are very difficult, if not impossible.

(a)

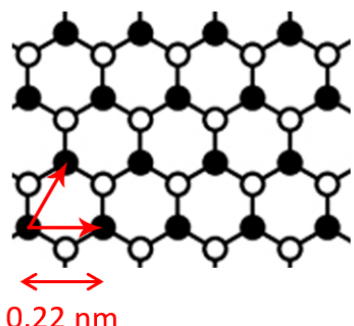

(d)

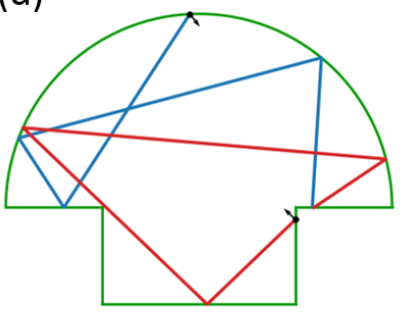

(b)

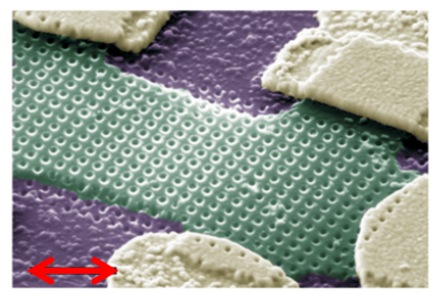

500 nm

(e)

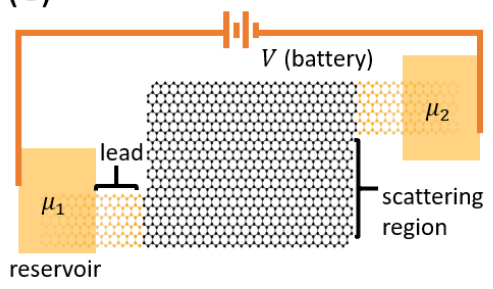

(c)

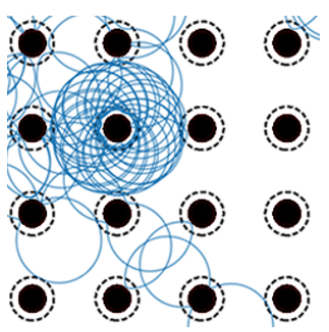

(f)
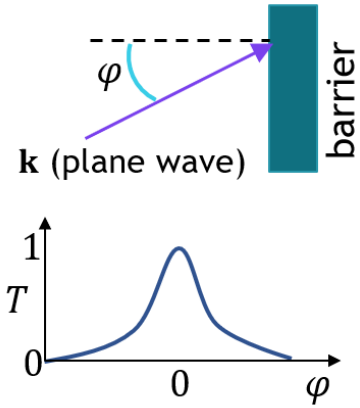

Figure 1.1: Example sketches of concepts and physical systems treated in this dissertation. (a) Graphene honeycomb lattice, with black and white dots denoting the two sublattices (see sec. 2.3). (b) Experimental realization of a graphene antidot superlattice (the green colored part), which is an artificial array of periodic repelling disks on top of a graphene lattice. Taken with permission from [62]. (c) A dynamical system that models (b): an electron is propagating chaotically in a periodic array of repellers (the electron trajectory is blue, see sec. 2.1.2 for more). (d) The mushroom billiard with two example trajectories. (e) A sketch of the Landauer-Büttiker setup, with the scattering region representing a graphene nanodevice in the middle. (f) A sketch of the Klein tunneling. 


\section{Quantum theory for ballistic nanodevices}

Notice that the dynamical systems approach, as is discussed here, is concerning classical mechanics. Some properties of nanodevices can be best understood using classical models, but usually nanodevices have to be described quantum mechanically (owing to their tiny sizes). Although there are possibilities to extend classical mechanics to include features of quantum systems, as done for example in ray-splitting billiards [63, 64, 65], sometimes one has to perform simulations of quantum systems in a direct manner. The approach that is used in various parts of this dissertation is based on the Landauer-Büttiker formalism [66, 8]. There, a nanodevice is treated as a compact unit (commonly called the scattering region), in which electron wavefunctions go in, get scattered by the device, and then go out again. A sketch illustrating this is shown in Fig. 1.1(e), while more details on the formalism and its implementation are given in sec. 2.5. What is important for this introduction is that this formalism is able to provide the electron wavefunctions inside the nanodevice.

A purely quantum phenomenon which is quite often of relevance in electron transport is quantum tunneling. Quantum particles can propagate through classically forbidden energy barriers with the propagation described by specific quantum rules [67]. These rules typically state that the probability to propagate through the classically inaccessible barrier decays exponentially with increasing the barrier width and strength [68]. This is the case for example in silicon or GaAs based nanodevices. Graphene, however, obeys different rules, owing its special physical properties stemming from the hyper-relativistic (Dirac) dynamics. A peculiar and even exotic tunneling process takes place in graphene, which is named Klein tunneling [69, 70, 71], after Oscar Klein, the first to study the effect in detail [72]. According to Klein tunneling, the probability of an electron in graphene to go through a potential wall depends strongly on the angle of incidence of the electron on the wall. Specifically, it holds that $T \sim \exp \left(-\gamma \sin (\phi)^{2}\right)$, as sketched in Fig. 1.1(f), with $\gamma$ a factor containing the parameters of the potential. Thus, the transmission is higher if the electron hits the barrier at normal incidence, while it becomes significantly smaller for oblique angles. It is crucial here to see that if $\phi=0$ (i.e. normal incidence on the barrier) then $T=1$ and thus we have perfect transmission of the electron. This is important, because this property holds irrespectively of $\gamma$, the details of the potential (i.e. width, strength, etc.). This may have profound effects in real world applications, as on the one hand it inhibits a straight-forward application of the operating principle of the transistor, while on the other it significantly reduces disorder-induced localization [71].

Quantum tunneling typically depends on the "angle of incidence" and this dependence is even more crucial for Klein tunneling. The angle of incidence may be possible to define for a trivial wavefunction as the wavevector angle [71], but it is not at all obvious to define for a complex wavefunction, as is the ones that exist inside a nanodevice like in the sketch of Fig. 1.1(e), since there there cannot be only a single angle of incidence. Furthermore, even for a simple device, although possible to define in the quantum sense (wavevector angle), the angle of incidence concept has a classical intuition. Two immediate and natural questions arise. Firstly, how can one define, and also obtain, such a concept as the angle of incidence in a complex quantum wavefunction? Secondly, how can one connect the quantum concept of Klein tunneling with the classical intuition of the phase space (position, momentum, velocity) through this angle of incidence? These specific questions may be new, because graphene is a relatively new material, but in a general sense such questions are not all that new; they fall under the concept of quantum-classical correspondence.

Quantum-classical correspondence is the train of thought that tries to connect the quantum realm with the classical world that we live and see on a daily basis. Even though quantum and classical phenomena can be wildly different, they are after all part of the same physical reality, 
and classical and quantum mechanics try to understand the same universe, just on different scales. Therefore, attempting to connect the two sides of the same coin is well justified [73]. The first discussion on the topic was by Niels Bohr [74] when he formulated the correspondence principle: quantum mechanics should reproduce classical physics in the limit of large quantum numbers (e.g. large energy levels, large orbits, etc.). Although heuristic, the principle is supported by Ehrenfest's theorem [75]. A more rigorous entry on the subject was offered by Wigner in 1932 [76]. Wigner came up with a rigorous way to transform a wavefunction into a probability distribution over momenta and positions, a construct now called the Wigner function [73], which takes as an input a wavefunction $\psi$ and returns a distribution $P$ on the phase space. Even though this seems to be the perfect way to transform quantum mechanics into classical mechanics, simply by taking $P$ as a probability distribution, there is a catch. $P$ can have negative values (owing to quantum interference) and thus is not so straightforward to interpret classically.

A similar approach to the Wigner function is provided by the Husimi function, first introduced by Kodi Husimi in 1940 [77]. The Husimi function is the Weirstrass transform of the Wigner function and thus retains all the rigorous properties with regards to derivations from quantum mechanics [78]. It has the advantage, however, that is has no negative values and thus it is much easier to interpret as a probability distribution over the classical phase space (for more see sec. 2.6). Husimi functions have been used extensively in physics, being a standard tool in quantum optics [79, 80, 78], and ocean acoustics [81]. The field that takes most advantage of Husimi functions is definitely quantum chaos [82, 83, 84, 85, 86, 87, 88, 89, 90, 91], a field of physics devoted to studying the quantum-classical correspondence in systems with complex dynamics. Given the discussion and questions raised in this subsection, one would expect Husimi functions to have been used extensively in condensed matter (where study of nanodevices is a subpart of), since they can give intuition on concepts like the angle of incidence of a quantum wavefunction. Surprisingly however, this is not at all the case. Only very recently Mason et al. introduced the Husimi functions for studying nanodevices [92, 93, 94], in an approach aiming to visualize classical paths inside the device. More in-depth analysis of classical concepts (e.g. angle of incidence and the phase space structure) and their definition given a quantum wavefunction are currently lacking in this field.

\subsection{Thesis synopsis and outline}

In this cumulative thesis I theoretically studied graphene-based nanodevices and ballistic electron transport within them, discussing the topics and answering the questions raised in the introduction section. The thesis is divided into two main parts, the first being classical theory while the second being quantum theory, with emphasis on semiclassical approaches (quantum-classical correspondence). The first part of the thesis is heavily motivated by recent graphene experiments, that show puzzling measurements of the resistance versus the magnetic field strength in an antidot superlattice (Fig. 1.1(b)) [41, 62]. Understanding and providing a theoretical background of these experiments required finding the inherent timescales of the dynamical system that represents the nanodevice (Fig. 1.1(c)). In addition, strong analytic connections were made between characteristic timescales in dynamical systems and the volume of sets in the phase space, and the impact of volume conservation of Hamiltonian mechanics was demonstrated. The second part is motivated by the questions raised in the final section of the introduction. It discusses how to obtain a (semi-)classical intuition of complicated quantum phenomena special to graphene, like Klein tunneling, using the Husimi function in both the absence and presence of magnetic fields. The significant contributions of this part of my thesis was firstly to show that the Husimi function can be very useful in condensed matter as a tool to understand nanodevices. This is because it provides an intuitive framework to understand concepts like the angle of incidence, 
but also because it allows measuring complicated concepts (like intervalley scattering, see below) straightforwardly. In addition, I extended the Husimi function for electrons moving in magnetic fields, and used it to study Klein tunneling in magnetic fields, which is not yet fully understood by the graphene community.

Chapter 2 provides the theoretical background necessary to understand the papers that make up my dissertation. The focus is specifically on the physics and methods relevant for this thesis. For example, antidot superlattices, billiards, graphene, Klein tunneling and the Husimi function are discussed in more detail and from a more theoretical perspective. Besides providing a background, this chapter also provides more context as well as more specific motivation to the questions discussed in the introduction.

Chapter 3 is the first paper that composes this dissertation. There we simulate a dynamical system representing an antidot superlattice (ADSL). The magnetoresistance of an ADSL shows characteristic peaks for magnetic field values where the cyclotron orbit is commensurable with the geometry of the device. Motivated by puzzling recent experiments with graphene ADSLs, which show the same characteristic peaks in a regime that shouldn't be possible, we analyze the timescales of the ADSL and find out which is the important inherent timescale relevant for the magnetotransport experiments. We then explain these experiments by understanding (and analytically prooving) that this characteristic timescale is reduced at the commensurable magnetic fields, simply due to the characteristic phase space volume conservation of Hamiltonian mechanics. In doing so, we also resolve a long-standing controversy on the origin of the magnetoresistance peaks in an ADSL. This chapter closes with unpublished results about the Hall effect of an ADSL, and highlights what gave us the insight to understand which is the important timescale, relevant for the experiments.

Chapter 4 presents a scientific software to simulate and analyze dynamical billiards, that I developed and published during my dissertation: DynamicalBilliards.jl. This software has been used extensively in the first two papers of this thesis, but it is also being used by other members of the scientific community. In the chapter I briefly overview the software, discuss the advantages of using it and point out some unique features that other software do not possess.

Chapter 5 is the second paper that composes this dissertation. There we analyze the Lyapunov exponent of several diverse billiards. This exponent is a timescale directly linked with the chaotic dynamics of a billiard. Motivated by the first paper (chapter 3), we link the exponent with the volume of the chaotic phase space. This not only provides a deeper, more fundamental understanding of how this chaotic timescale is linked with the properties of the phase space, but it also readily connects the timescale with the parameters of the billiard. This is because the phase space volume is very often determined analytically from the parameters of the billiard, or at least straightforwardly from numeric simulations. A result of broad interest from this work is that the Lyapunov exponent generically has a contribution that is inversely proportional to the chaotic phase space volume. This result is also backed by strong analytic arguments, assuming only the phase space volume conservation (which is a property of all Hamiltonian systems).

Chapter 6 is the third paper that composes this dissertation. In this chapter a transition is made from classical to quantum mechanics, which is motivated in more detail in sec. 2.4. The paper implements the Husimi function to study the Klein tunneling effect discussed in the introduction, as well as intervalley scattering, another quantum process that is inherent 
to graphene. In this work we showed that the Husimi function is an excellent tool fitting to be used in the field of condensed matter, as it can be used both for intuition but also for uncovering fundamental information. More specifically, we showed that using the Husimi function allows one to reproduce theoretical predictions on Klein tunneling, and to quantify Klein tunneling in scenarios intractable by theory. Furthermore, we showed how analyzing the Husimi function can provide a quantitative measure of intervalley scattering (see sec. 2.3), which in essence is scattering in momentum space due to either the device geometry or the electrostatic potential. Surprisingly, electrostatic potentials like pn-junctions have strong asymmetry in their intervalley scattering. Finally, we demonstrated that a specific termination of the graphene lattice (called armchair) is by far the strongest intervalley scatterer in graphene, a result that was often suggested theoretically in the literature but not discussed much quantitatively.

Chapter 7 is the final project of this dissertation. In this chapter we firstly extend the Husimi functions for electrons moving in magnetic fields, to properly account for energy considerations. We perform numeric simulations that validate our extension, and also show that it is possible to recover localized wavepackets (to create e.g. point particles) in magnetic fields in graphene devices through the Husimi function. Then, we use this new tool to study Klein tunneling in magnetic fields, which is an effect that has been studied little and seems to be lacking understanding. We show that the currently standing theory for Klein tunneling in magnetic fields cannot explain the numeric results of simple quantum transport simulations in pn-junctions.

Chapter 8 is the closing chapter of the dissertation. It summarizes the results of the various projects that compose my dissertation. A discussion of potential applications of my results, as well as direct continuations of some of the projects concludes the thesis. 


\section{Fundamental Concepts}

The goal of this chapter is twofold. On one hand, it introduces the fundamental concepts, underlying physical principles, governing mathematical laws and relationships between the physical systems that are treated in this dissertation. The concepts that are discussed here wellestablished, and therefore only a brief overview will be given. On the other hand, the chapter provides more context and more motivation to the specific questions and problems that are treated in this thesis as well as justification on the choices of what modeling approaches to use.

\subsection{Nonlinear dynamics of antidot superlattices}

The physical systems that motivated the present thesis are called graphene antidot superlattices (Fig. 2.1). These are in essence two dimensional nanodevices with a external modification done on top of the crystalline lattice. Before going into the details of the antidot superlattices however, it is best to review the basic relationship between current and resistance in a two dimensional electronic system in the presence of magnetic fields [8].

To experimentally measure magneto-transport (i.e. current and resistance in the presence of a magnetic field), one typically uses the Hall bar setup [8], shown in Fig. 2.1(a). A current is applied across the device, and then one can define the magneto-resistance $R_{x x}=V_{L} / I$ and the Hall-resistance $R_{x y}=R_{H}=V_{H} / I$. The classical Drude theory for conductors says that the magneto-resistance of a two dimensional electron conductor is independent of the magnetic field $B$, while the Hall-resistance is linearly proportional to it [27]

$$
R_{x x}^{(D)}(B)=\frac{m}{|e|^{2} n_{s} t_{m}}, \quad R_{x y}^{(D)}(B)=\frac{B}{|e| n_{s}} .
$$

Here $n_{s}$ denotes the electron density (for graphene given by eq. (2.3.14)), while $t_{m}$ stands for the mean free time, which for the purposes of this thesis is the average amount of time an electron moves before it interacts with noise-inducing factors (e.g. impurities or defects) and randomizes its own velocity. It was later discovered that for very strong magnetic fields the quantum Hall effect (QHE) occurs [95, 96]. There, the Hall-resistance increases in quantized steps, while the magneto-resistance follows strong oscillations versus $B$, called Shubnikov-de Haas oscillations, see [8] for more. This thesis is concerned with magnetic field values below the QHE regime.

\subsubsection{Antidot superlattices}

Additional effects arise when one modifies the two-dimensional conductor via means of a superlattice: a periodic modification, with periodicity at a scale much larger than that of the regular crystalline lattice of the material that composes the nanodevice. This modification typically acts as an electrostatic potential. One such example is a rectangular or hexagonal lattice of repulsors, called antidot superlattice (ADSL), which is shown in Fig. 2.1(b, d). Holes are "drilled" in the material periodically, typically using electron beam lithography [97], and these holes act as repulsive centers for the electrons [36]. Fig. 2.1(c) shows is an example potential landscape that is probed by the electrons. 
Such ADSL modifications were first done in the 1990 by three groups. D. Weiss and colleagues [97] used electron beam lithography while K. Esslin and P. Petroff [98] used ion bean implantation to create the antidots by "drilling" holes in the material. In these cases the antidots are absence of (or heavily damaged) material. A. Lorke et al. [99] created antidot superlattices via means of electrostatic modulation using lateral gates. In this case the antidots are formed by the electrostatic potential, instead. In all three cases the antidots are perceived by the electrons as repulsive centers either because they are physically inaccessible, or because the potential barriers are unpenetrable with quantum tunneling due to their high strength and width. In addition, Refs. [97, 98, 99] used GaAs heterostructures as the material basis and fabricated ballistic devices (i.e. with sufficiently large mean free time). Since then, antidot superlattices have been created in a wide range of materials $[100,101,102]$ including graphene $[62,41]$ and very recently topological insulators [42]. ADSLs have even been used to study composite fermions, which are particles that lead to the fractional quantum Hall effect, see e.g. [103]. Reviews on antidot superlattices in semiconductor heterojunctions and graphene can be found in [104] and [105] respectively.

Assuming that we are in the small magnetic field regime and at low temperatures, where the Drude picture should still hold, all aforementioned experimental realizations of antidots show that the Drude picture actually breaks down. Instead, interesting structures occur in both the magneto-resistance $R_{x x}$ as well as the Hall-resistance $R_{x y} . R_{x x}$ displays a series of peaks at characteristic magnetic field values, which we show in Fig. 2.2 for three different materials. At the same values $R_{x y}$ displays non-quantized steps (but only in experiments with sufficiently small mean free time [62]). The reason these ADSLs are a very interesting system is exactly because of the aforementioned peaks in the magneto-resistance.
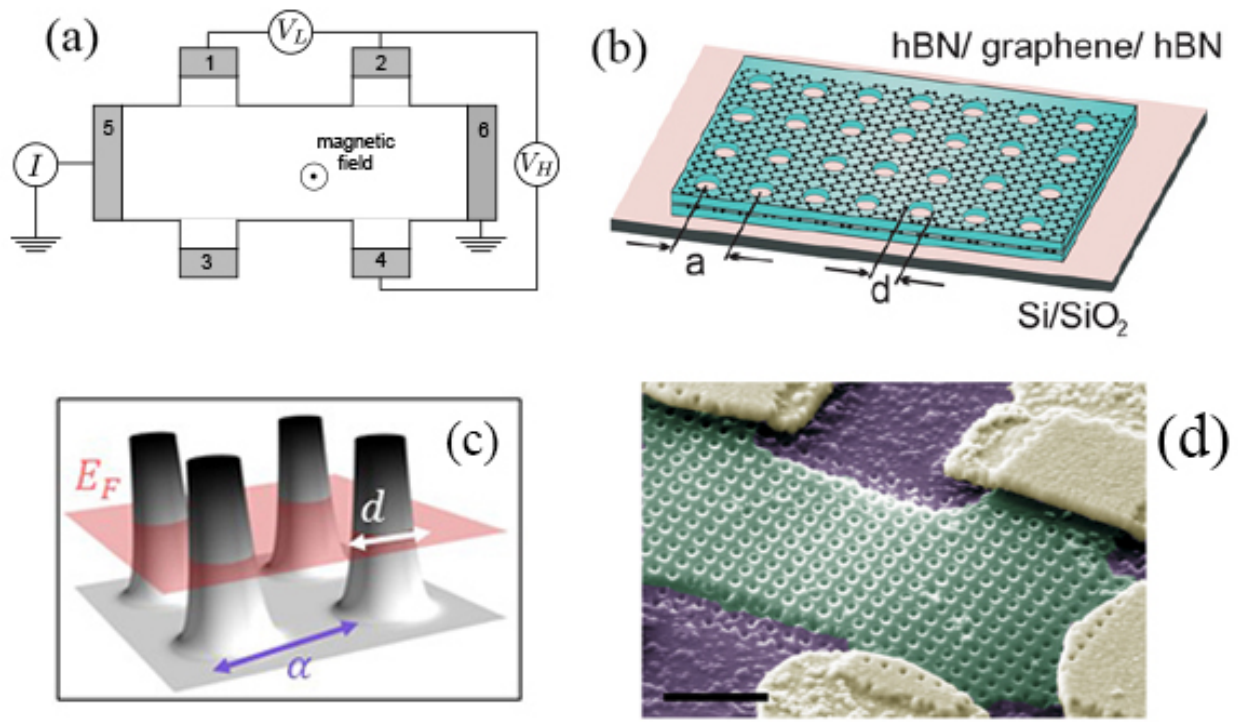

Figure 2.1: (a) Hall-bar experimental setup. (b) Sketch of an antidot array consisting of periodically arranged holes etched into a graphene nanodevice, taken from [62] (replicated with permission). (c) The corresponding potential landscape probed by the electrons in the ADSL. The electrons move at the Fermi energy, depicted by a semitransparent red plane. (d) False-color scanning electron micrograph of a real graphene antidot superlattice sample. Here, the heterostructure is shaded in green, the Cr/Au contacts yellow, and the Si/SiO2 substrate violet. Scale bar length $500 \mathrm{~nm}$. Reproduced with permission from [62]. 


\subsubsection{Nonlinear dynamics}

Because the length scales in antidot superlattices involved are on the order of 10 to 100 nanometers, one would expect that quantum mechanics plays a big role in the observed physics. Yet, ADSLs are devices that display a large range of classical effects [104], even though their length scales are in a regime where quantum mechanics is typically important [8] (this is also possible because in the ballistic experiments of $[97,98,99]$ the Fermi wavelength was comparable with the characteristic lengths of the nanodevice). After the first fabrication of ADSLs it was immediately realized that the magnetic field values where the $R_{x x}$ peaks occur are very specific. In fact, these peaks occur when the classical cyclotron radii can (approximately) enclose an integer number of antidots without collision (the cyclotron radius is inversely proportional to the magnetic field $r \sim 1 / B)$. Such cyclotron examples are shown in the insets of Fig. 2.2(a, b) but also in Fig. 2.3. Because of this condition, the magneto-resistance peaks are referred to as Commensurability Peaks (CPs). Another interesting effect is the "quenched Hall effect" that will be discussed after presenting the first paper of chapter 3, in sec. 3.1. Notice that the concept of the cyclotron radius (i.e. the radius of the circular motion an electron performs in the presence of a constant magnetic field) is an entirely classical concept.

Many research groups were able to reproduce the CPs using classical mechanics [36, 106, 107, 108, 104, 109]. Most attempts to explain the origin of the CPs were based on the nonlinear dynamics of the electrons in the lattice and the nonlinear resonances with respect to the commensurable orbits. However, the exact mechanism for the origin of the CPs was controversial in the literature because different groups were claiming different kind of resonances to be important (for more details please see the in-depth discussion happening in the first paper, chapter 3).

At this point it is best to define and explain the term "nonlinear resonance". The dynamics of the electrons in an ADSL with a perpendicular magnetic field can be modeled within the single electron picture by a simple dynamical system introduced by Fleischmann, Ketzemerick and Geisel [36]

$$
\begin{aligned}
\dot{x} & =v_{x} \\
\dot{y} & =v_{y} \\
\dot{v}_{x} & =2 \sqrt{2} B v_{y}-\partial U / \partial x \\
\dot{v}_{x} & =-2 \sqrt{2} B v_{x}-\partial U / \partial y \\
U(x, y) & =U_{0}[\cos (2 \pi x) \cos (2 \pi y)]^{\beta}
\end{aligned}
$$

where $x, y$ is the position of the electron (the antidots are located at $x, y=0 \bmod 1), v_{x}, v_{y}$ the velocity, $B$ the magnetic field and $U$ is the potential landscape created by the superlattice.

(a)

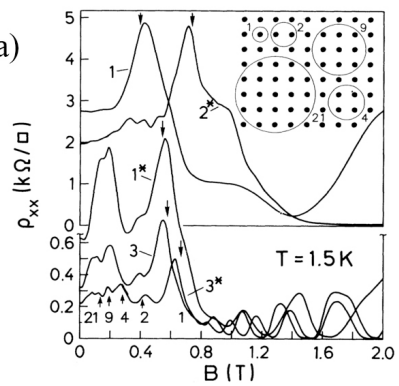

(b)

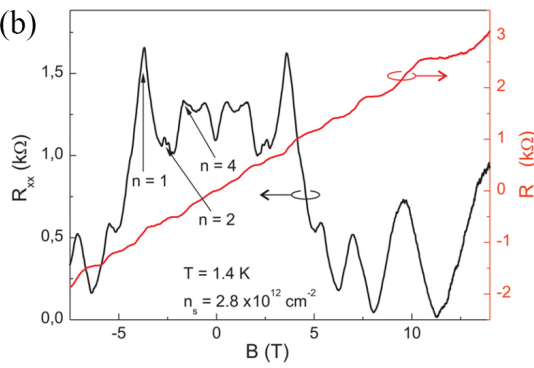

(c)

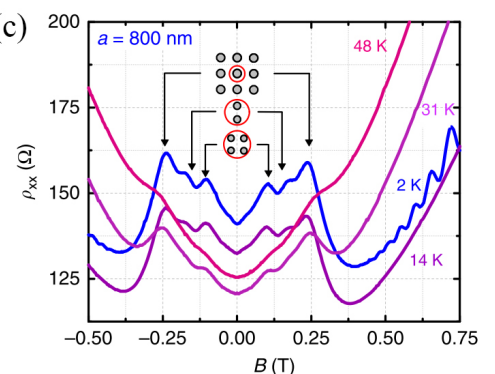

Figure 2.2: Commensurability peaks (CPs) as a universal feature of antidot superlattices (ADSLs). The subplots show magneto-resistances of ADSLs patterned into different materials. (a) 2DEG of semiconductor heterostructures (from [97]), (b) graphene (from [62]), (c) topological insulator (from [42]). All figures reproduced with permission. 
I will call this dynamical system the FKG model in the following. For the current discussion the parameters $U_{0}, \beta$ are of low importance for the dynamics. This means that in principle the system has a single parameter, the magnetic field $B$, which has dramatic impact on the resulting dynamics. The system is also Hamiltonian since it is just a representation of Hamilton's equations of motion for $H=(\mathbf{p}-\mathbf{A})^{2}+U$ (with $\mathbf{A}$ the vector potential). Besides the FKG model, one can also use the periodic Sinai billiard (PSB) [110, 97, 111], Fig. 2.4, as a model for the ADSL which is equivalent with the FKG model with $\beta \rightarrow \infty, U_{0}=1 /$ const. $^{\beta}$. This results in a simplified model, which cannot reproduce the experimental measurements with as high accuracy as the FKG model. Nevertheless, it still reproduces the fundamental properties of the magnetoresistance and thus is fitted to examine in detail fundamental concepts (see chapter 3).

The orbits shown in Fig. 2.3 are the result of straight-forward numeric integration of (a modified version of) the FKG model. Once we have the timeseries of the model's variables, it is possible to calculate the resistances using the Kubo formalism [112]. Following [36] the result is

$$
\begin{aligned}
\sigma_{i j} & \sim \int_{0}^{\infty} e^{-t / \tau_{i}}\left\langle v_{i}(t) v_{j}(0)\right\rangle_{E_{F}} d t, \\
R_{i j} & =\frac{\sigma_{i j}}{\sigma_{x x}^{2}+\sigma_{x y}^{2}}
\end{aligned}
$$

with the conductivities $\sigma_{i j}(i j=x x$ or $x y)$ and the magneto- and Hall-resistivity $R_{x x}$ and $R_{x y}$, respectively (modulo some geometry prefactor). $C_{i j}(t) \equiv\left\langle v_{i}(t) v_{j}(0)\right\rangle_{E_{F}}$ is the velocity correlation function (VCF), averaged over the available phase space at the Fermi energy. Here $\tau_{i}$ represents the impurity scattering time, also called the mean free time.

The solutions to the FKG model are typically chaotic. However, the model also has periodic solutions in the phase space for specific magnetic field values [36]. These periodic orbits are of two kinds, both shown in Fig. 2.3. The first kind are the collision-less electron orbits that were already discussed. The second are the "skipping" electron orbits, that hop periodically across the

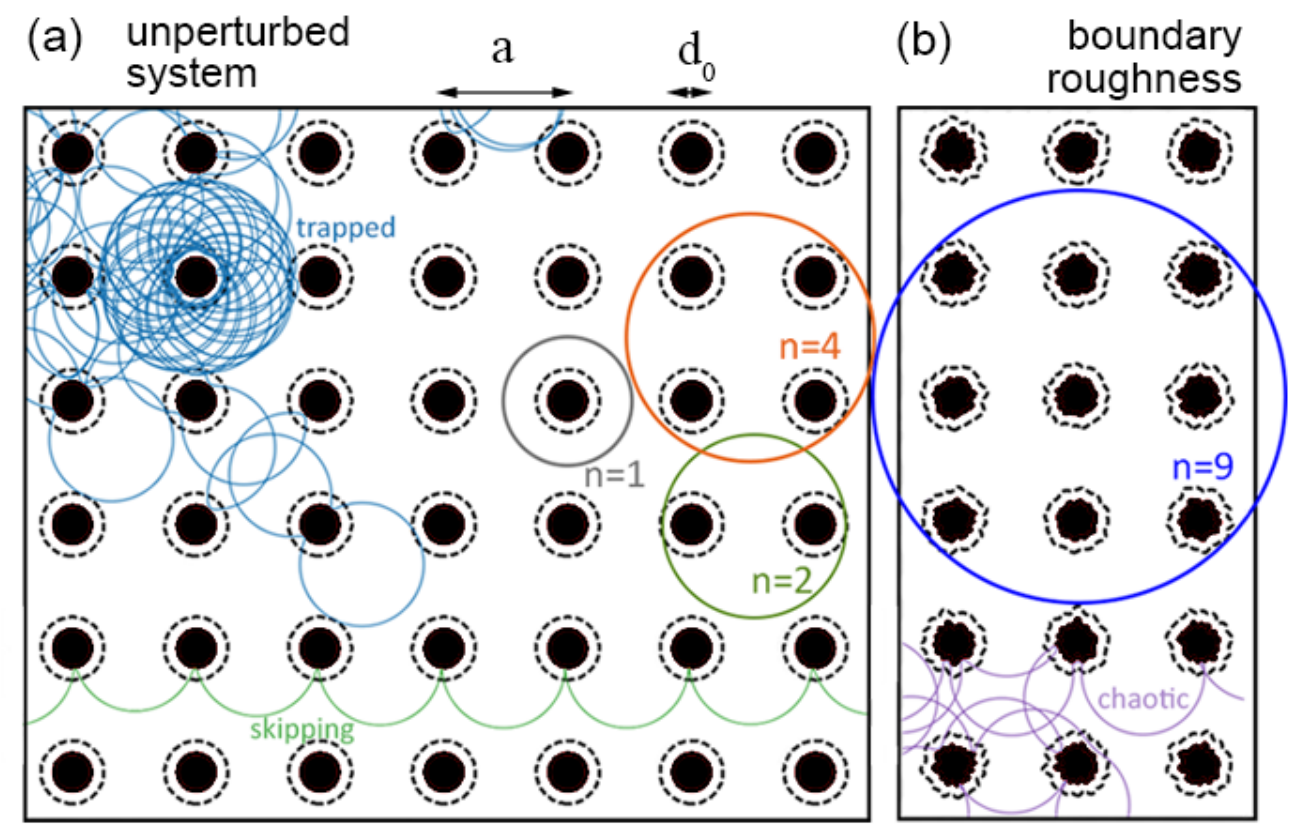

Figure 2.3: Replication of Fig. 2 of the first manuscript of my thesis, ch. 3. The Figure shows the antidot superlattice (black filled disks), the two types of periodic motion (collision-less electron orbits at different commensurable magnetic fields $n=1$ to 9 as well as skipping orbits) and typical chaotic motion (both just "chaotic" as well as trapped). The boundary roughness (right part of the figure) is not discussed in this introduction. 
different antidots. Notice that the collision-less electron orbits, also called pinned orbits in the literature [36] (in the PSB the skipping orbits are unstable). These periodic orbits are composing the nonlinear resonances of the system. In a nonlinear dynamical system (that ADSLs are), the chaotic electrons can "resonate" with these nonlinear resonances and follow their dynamics for sufficiently large time spans until returning again to the "chaotic" dynamics (for an exact description of how this resonance can occur, please see [36] or one of the textbooks on nonlinear dynamics [60]). These interplay of the chaotic electrons with the nonlinear resonances are what the different research groups used to explain the origin of the CPs, but there was a controversy since different groups were focusing on different resonances. Regardless, the fundamental claim was that the CPs are a result of the nonlinear resonances in the dynamics of the ADSL. At this point one has to realize that for this claim to make sense, the mean free time of the electrons inside the real device has to be several times larger than the resonance period, which was indeed the case for the original ADSL systems fabricated in semiconductor heterostructures [97].

\subsubsection{Graphene antidot superlattices}

Two recent experimental papers were able to fabricate ballistic nanodevices of ADSL based on graphene $[62,41]$ that had sufficiently large mean free time that allowed one to resolve magnetoresistance features like the CPs in Hall-bar measurements. This is of interest, because electron motion in graphene has different basic transport properties than in standard semiconductors. Specifically (and more details on this are in sec. 2.3) the carrier motion follows hyper-relativistic dynamics instead of classical, i.e. the kinetic energy of the particles has the form $K \sim|\mathbf{p}|$ instead of the common case of $K \sim \mathbf{p}^{2}$ (here $\mathbf{p}$ is the electron momentum). In my M.Sc. thesis [113] I extended the FKG model to accommodate the hyper-relativistic dynamics, and showed that the fundamental aspects of the model remained intact: the resonances, the commensurability peaks and other properties only changed slightly in numerical value but not qualitatively.

The bigger challenge that these two new experimental papers brought though was not the relativistic dynamics, but the fact that the mean free time in these devices was extremely small. If $t_{a}$ is the average time an electron needs to move from one antidot to the next one, the experiments had a mean free time of at most $\tau_{i}=3 t_{a}$. Why is this a challenge? The smallest period of the aforementioned resonances is $\pi t_{a}$ (for details see ch. 3). If one wants to use the resonances to explain the origin of the CPs, the mean free path should be much larger than the period of the resonance. Yet, in both the new experiments in graphene ADSLs as well as preliminary simulations done in my M.Sc. thesis, it was clear that CPs can exist when the mean free time is $3 t_{a}$. This simply means that the old theories cannot explain the origin of the CPs, at least not at the fundamental level. "What is the origin of the CPs" is exactly what the first paper of this thesis answers.

\subsubsection{Origin of commensurability peaks}

Finding the origin of the commensurability peaks (CPs) was the first project of my dissertation. The full details of this are described in chapter 3, using the published paper as well as unpublished results that give some insight into the process that lead to answering the question. I believe it is worthy to provide here a conceptual summary and sketch the core ideas that lead to the answer, because it further motivates the remaining thesis. Basically, the CPs exist due to volume conservation.

Three ingredients are necessary to understand the origin of the CPs. The first ingredient is the volume-preserving nature of the phase space dynamics for an ADSL. Within the framework of classical mechanics, the phase space of (for example) the FKG model is mixed: chaotic motion coexists with regular motion for different initial conditions, at least for the commensurable magnetic field values. For other magnetic field values the phase space is entirely chaotic. To understand this better please see Fig. 2.4. There one can clearly see that for some values of $B$ 
it is impossible to find periodic orbits. For some other values of $B$ though (the commensurable values), both chaotic and regular orbits exist in the phase space.

Besides the fact that the phase space is mixed, the FKG model (and also the periodic Sinai billiard, PSB) conserve phase space volumes, because they are Hamiltonian systems [60]. In addition, these systems have the interesting property that their total phase space volume is independent of the magnetic field $B$. This has the implication that both regular and chaotic trajectories compete for the same phase space volume. Therefore, if the amount of regular trajectories is increased from zero (non-commensurable magnetic field) to a nonzero value (at a commensurable magnetic field) the total volume the chaotic orbits can occupy will necessarily decrease.

The second ingredient of this sketch of the origin of the CPs is how the resistance of the ADSL is connected with the electron motion in the FKG or PSB models. What turns out to be the case is that it is possible to connect the resistance $R_{x x}$ with the mean collision time $\kappa$ of the electrons in the ADSL (skipping many details which are enclosed in chapter 3 ). $\kappa$ simply notes the average amount of time an electron needs to travel from one collision with one antidot to the next collision with the next antidot. Keep in mind that $\kappa$ and $\tau_{i}$ (the mean time between impurity scatterings) are independent and model completely different physical processes.

To be more specific, the magnetoresistance has a contribution inversely proportional to the mean collision time $\kappa$. A valley in the curve $\kappa$ vs. $B$ corresponds to a peak in $R_{x x}$ vs. $B$. In Fig. 4 of the manuscript of ch. 3 it is clearly shown that $\kappa$ has valleys at the commensurable magnetic fields, where the magnetoresistance has peaks. To conceptualy understand why this should be the case, one does not need the details of the paper. On the most basic level, resistance is how difficult it is for the electrons to move through a device. It makes sense that, at least on average, the more frequently the electrons collide inside the device (i.e. the lower the $\kappa$ ) the higher the resistance of the device should be. And to aid this conceptual argument, we can also

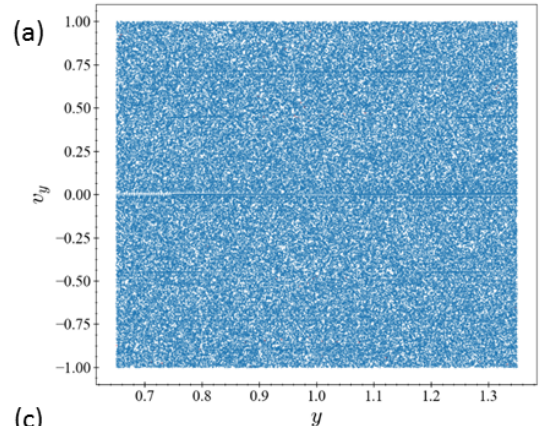

(b)
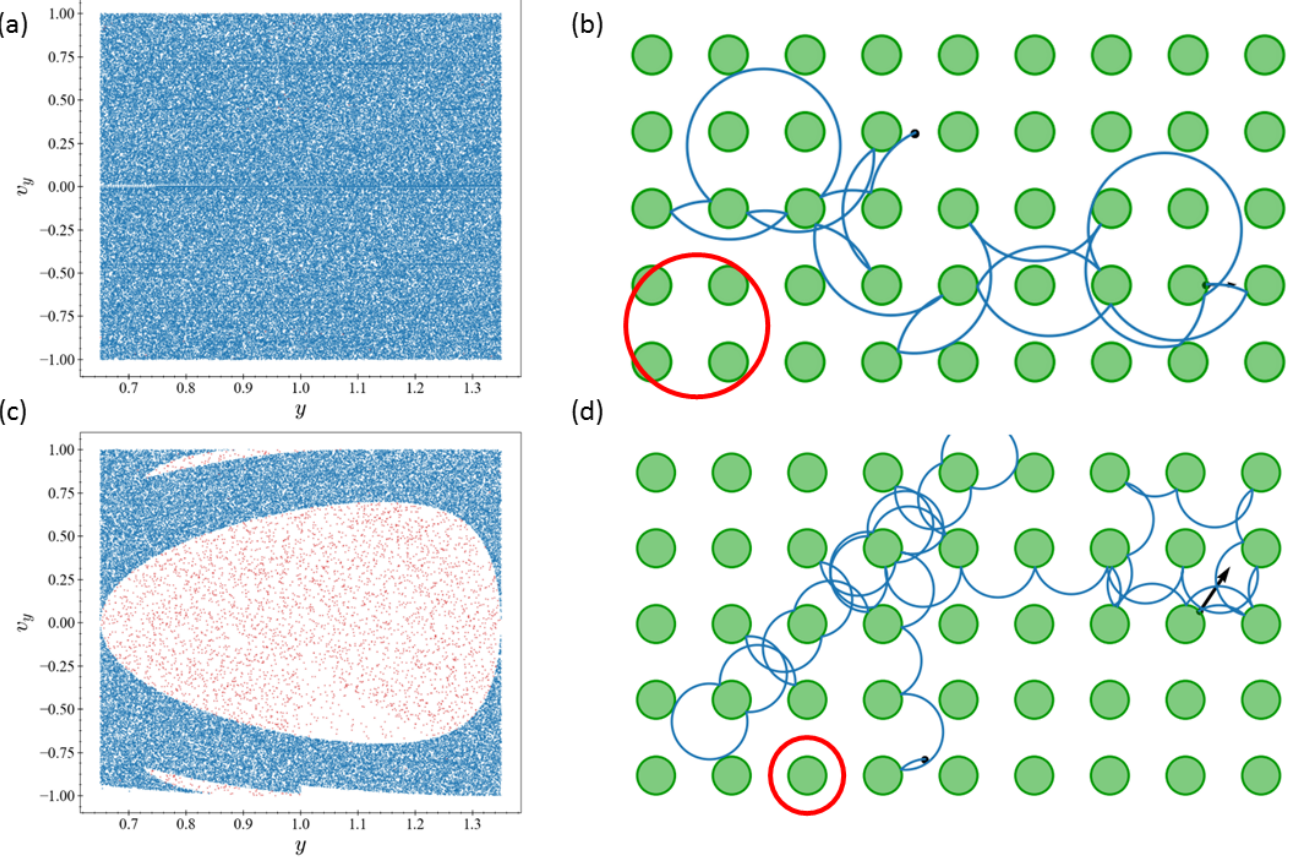

(d)

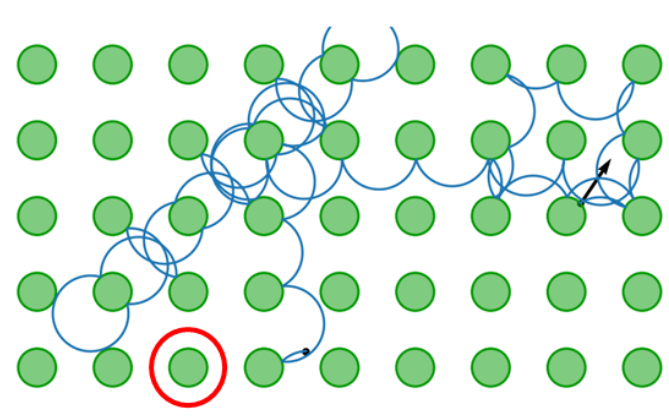

Figure 2.4: Mixed nature of the phase space of the FKG model or the periodic Sinai billiard (in the Figure the billiard is shown, similar results are obtained for the FKG model). (a) Poincaré surface of section (PSOS) and (b) movement in the ADSL for $B=0.4$ (non-cummensurable value). $(c, d)$ same for $B=1.0$ (commensurable value). Chaotic orbits are colored blue in the PSOS while periodic orbits are colored red. Notice that in $(b, d)$ a red circle indicates the cyclotron motion. In (b) it is impossible to fit such a circle and obtain periodic motion. 
invoke the basic Drude formalism of eq. (2.1.1) that generically states that magnetoresistances are generically inversely proportional with mean free times.

By now it is established that the reason $R_{x x}$ has the CPs is because the mean collision time in the ADSL $\kappa$ becomes smaller at the commensurable magnetic fields, which is also the magnetic field values where periodic orbits exist. But the question now is, why should $\kappa$ be reduced when regular orbits exist? The chaotic orbits (which contribute to electron transport and whose average time between collisions is $\kappa$ ) do not necessarily interact with the periodic orbits. The third and final ingredient in understanding the true origin of the CPs is Kac's lemma $[114,115,116,117]$, which is a universal property of measure preserving maps. Kac's lemma is extremely simple and astonishingly general. It states that for any measure preserving map $f: M \rightarrow M$, which preserves a measure $\mu$, and any set $S \subset M$ it holds

$$
\left\langle n_{S}\right\rangle=\frac{\mu\left(M_{a c c}\right)}{\mu(S)} .
$$

$M_{a c c}$ is the accessible part of $M$ for trajectories starting in the set $S$ while $\left\langle n_{S}\right\rangle$ is the average amount of iterations needed for a trajectory to return back to $S$ (starting to count immediately after exiting the set). Notice that it is guaranteed that the trajectories will at some point return to $S$ due to Poincaré's recurrence theorem [60]. A sketch that illustrates Kac's lemma is shown in Fig. 2.5. Notice that Kac's lemma is a direct result of measure preservation and requires no additional assumptions about the system (see [116] for a proof).

Why is Kac's lemma relevant for the magnetoresistance of an ADSL? Let $g_{c}(B)$ denote the portion of the phase space of the ADSL that is chaotic. This portion is at most 1 at the non-commensurable magnetic fields, and is reduced from 1 when periodic orbits exist (at the commensurable magnetic fields). What I was able to prove is that

$$
\kappa(B)=g_{c}(B) \kappa(0)
$$

i.e. that the mean collision time of the chaotic orbits in the ADSL is directly proportional to the chaotic phase space portion. This portion $g_{c}$ can also be interpreted as the probability that a random initial condition will be chaotic. The proof of eq. (2.1.10) is fully analytic, based on Kac's lemma, and is presented in the first paper in chapter 3. In essence this means that the mere existence of the periodic orbits affects the chaotic orbits indirectly, by reducing their average collision time simply by occupying some part of the phase space. Thus, again indirectly,
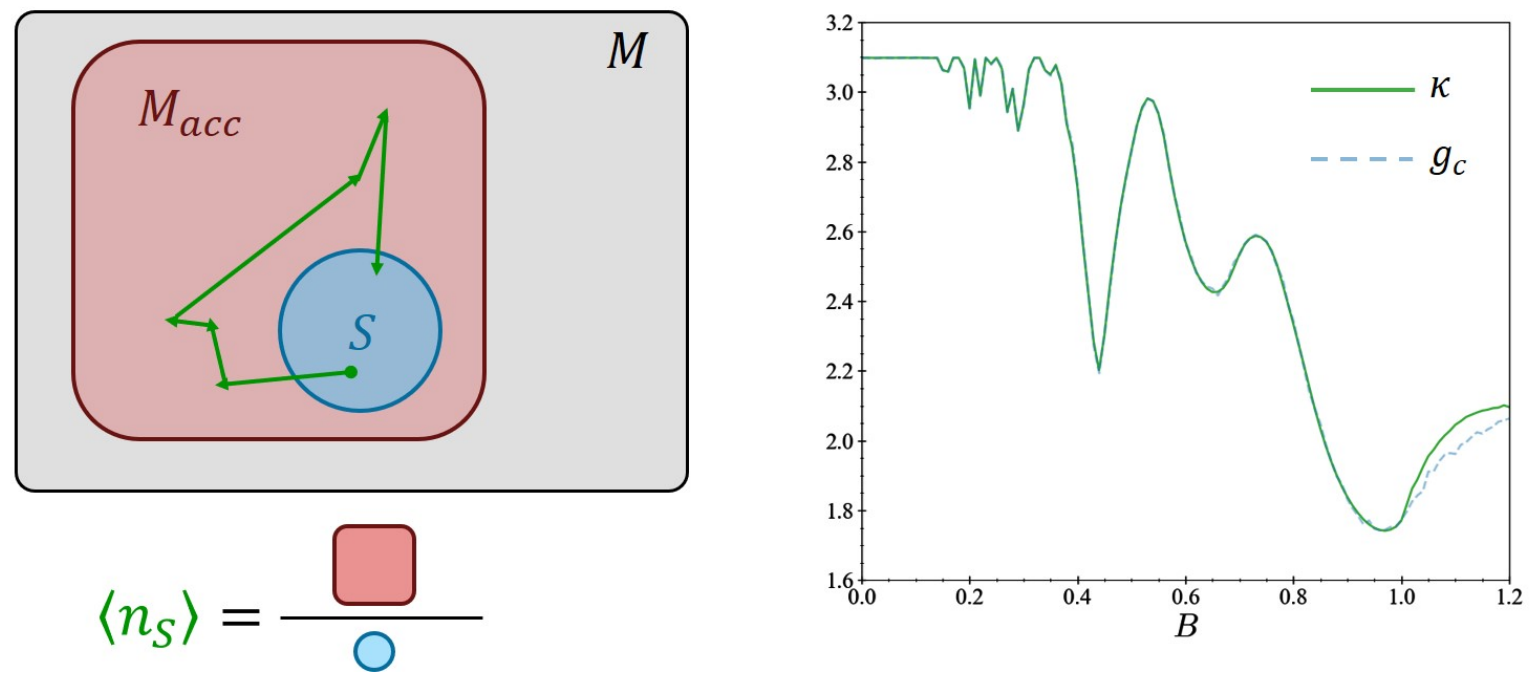

Figure 2.5: Left: A sketch of Kac's lemma. Right: Numeric computation of the mean collision time $\kappa$ in the periodic Sinai billiard and portion of the chaotic phase space $g_{c}$ (scaled to same maximum), versus the magnetic field. 
the presence of these periodic orbits, an entirely classical concept, leads to the commensurability peaks of the magnetoresistance.

\subsection{Lyapunov exponents in billiards}

\subsubsection{Motivation}

While explaining the origin of CPs in ADSLs, we uncovered a fundamental connection with a characteristic transport timescale in a dynamical system (specifically the PSB) and the way the phase space is affected by an external parameter (here the magnetic field): $\kappa(B)=g_{c}(B) \kappa(0)$. The ingredients that led to this relation were all derived purely from volume conservation. It seems valid that such relation could be generalized and phase space volumes could be connected with more than a transport timescale and in more systems than only the periodic Sinai billiard.

For several reasons, an excellent family of dynamical systems to test this hypothesis is billiards. Firstly, they are measure preserving and thus satisfy the necessary criterion for Kac's lemma. Secondly, billiards are conceptually simple to understand yet most of them display a plethora of interesting dynamics [118]. Thirdly, it is straight forward to connect distances in the phase space, with paths in the real billiard space, since the phase space variables of the billiard system are the position and the propagation angle. This provides an intuitive framework for one to conceptualize what mean return times mean (which is what Kac's lemma provides). Fourthly, billiards have been used extensively in many areas of physics, like ergodic theory [110, 119, 120], quantum chaos [47, 48], optical microresonators and laserss [49, 50] and room acoustics [121]) and also in condensed matter to model transport properties of electronic nanostructures such as quantum dots and antidot superlattices [122, 36, 97, 40, 41, 42, 43, 44, 45, 46] and even more specifically for graphene nanodevices $[51,46,52,53]$. This means that any general results we can find about billiards could have potential implications for various areas of physics and thus have a broad impact. Lastly, the software DynamicalBilliards.jl [123] (chapter 4) allowed us to examine any billiard of any shape with ease.

Thus, in the second paper of this dissertation (chapter 5), we studied billiards which have mixed phase space, so that phase space volumes can be tuned by an external parameter. The first billiard we looked at was the aforementioned periodic Sinai billiard [122, 110], the second was the mushroom billiard [124] and the third one was the inverse stadium billiard [125]. Fig. 2.6 shows the billiards as well as how their volume changes with a change in a parameter. Part of the standing hypothesis of this section is that phase space volumes should be connected with "more than a transport timescale". To put this vague concept into concrete terms, we decided to study the Lyapunov exponent of these billiards.

\subsubsection{Chaos and Lyapunov exponents}

The Lyapunov exponents are a set of numbers that characterize stability properties of the phase space, as well as quantify the chaotic motion, introduced first by A. Lyapunov [54]. A dynamical system has as many exponents as its dynamical variables, and thus a billiard has four. However, because billiards are Hamiltonian systems, one can show that $\lambda_{1}=-\lambda_{4}, \lambda_{2}=\lambda_{3}=0$. Thus one only needs to discuss $\lambda \equiv \lambda_{1}$, the maximum Lyapunov exponent. The way $\lambda$ quantifies chaotic motion is based on how quickly nearby trajectories separate (or converge) from each other as the trajectories evolve in the phase space $[126,58]$.

To intuitively grasp the concept of the Lyapunov exponent, imagine the following scenario, demonstrated in the sketch of Fig. 2.7: a ray of particles, all with the same velocity but a tiny difference in their initial position, are propagated at the same time in the periodic Sinai billiard. The initial maximum distance between the particles is $\delta=\delta_{0}$. As time progresses and the particles move according to the dynamics of the billiard, their distance $\delta$ changes. Because the Sinai billiard is indeed chaotic [110], their distance increases, as can be seen by the fact 
that the ray of particles expands. At some later point in time $t$ the distance has value $\delta>\delta_{0}$. The Lyapunov exponent quantifies how fast $\delta$ increases, with the formula $\delta=\delta_{0} \exp (\lambda t)$. In a non-chaotic system, the trajectories get closer with each other. In such a case we would have $\lambda \leq 0$, but chaotic dynamics always have $\lambda>0$. Typically, the Lyapunov exponent is calculated based on the evolution of perturbations in the phase space. A simple formula for this is [58]

$$
\lambda_{\boldsymbol{\Gamma}(0), \delta \boldsymbol{\Gamma}(0)}=\lim _{t \rightarrow \infty} \frac{1}{t} \log \frac{|\delta \boldsymbol{\Gamma}(t)|}{|\delta \boldsymbol{\Gamma}(0)|}
$$

with $\boldsymbol{\Gamma}$ being the phase space vector (for billiards a vector of the position and velocity of the particle) and $\delta \boldsymbol{\Gamma}$ a perturbation vector, evolving according to the evolution equations in tangent space, $\delta \dot{\boldsymbol{\Gamma}}=J(\boldsymbol{\Gamma}(\mathbf{t})) \cdot \delta \boldsymbol{\Gamma}$ where $J$ is the Jacobian matrix of the equations of motion.

\subsubsection{The Lyapunov exponent as a timescale}

For chaotic motion, $\lambda$ can be understood as the the inverse timescale that nearby trajectories need to separate and become uncorrelated. Thus, the larger $\lambda$, the more strongly chaotic is a system. An important fact is that for chaotic systems (or at least for the chaotic part of the phase space of a dynamical system), it holds that the value of $\lambda$ is an intrinsic property of the dynamics, which does not depend on the initial condition $\boldsymbol{\Gamma}(0)$.

This interpretation of $\lambda$ already motivates why we would choose $\lambda$ to test the hypothesis that phase space volumes could be generically connected with timescales beyond the specific case of the periodic Sinai billiard. The exponent is naturally representing with a chaotic timescale of the system. It seems valid therefore to assume (based on the findings of chapter 3 of course) that this timescale that the exponent represents can be connected with phase space volumes via Kac's lemma.

And indeed, in the second paper of my dissertation, we are able to show that in billiards, $\lambda$ has a leading contribution inversely proportional to the volume of the chaotic part of the phase space. For more details on this finding, I point to chapter 5.
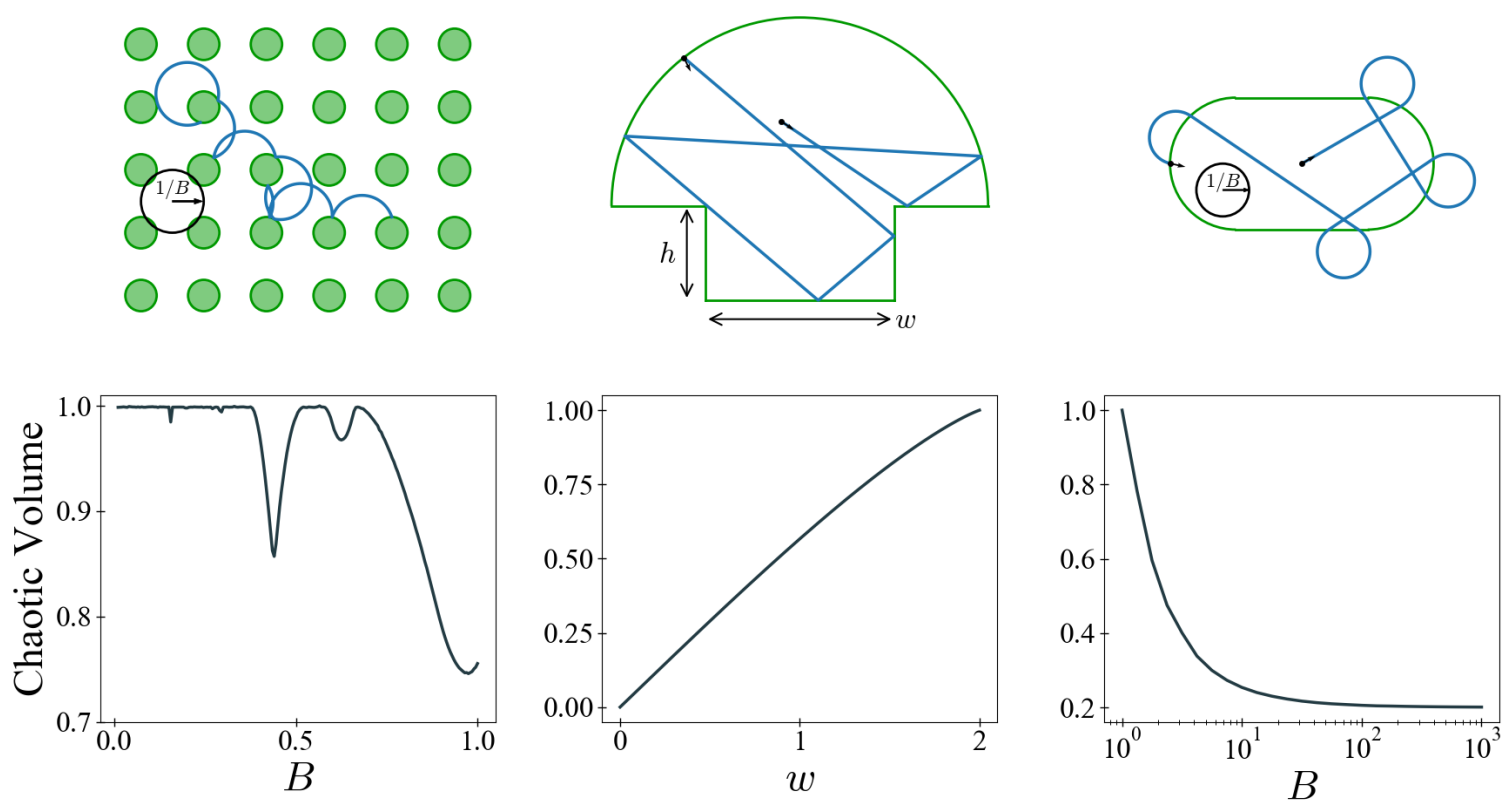

Figure 2.6: The periodic Sinai billiard (with magnetic field), the mushroom billiard and the inverse stadium billiard. Below each billiard their chaotic phase space volume is plotted (normalized to maximum value for simplicity). 


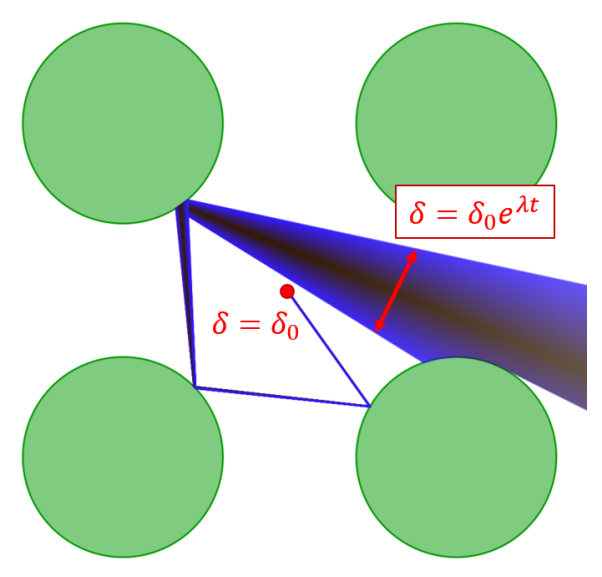

Figure 2.7: A sketch demonstrating the concept of exponential divergence of trajectories, as well as the Lyapunov exponent $\lambda$.

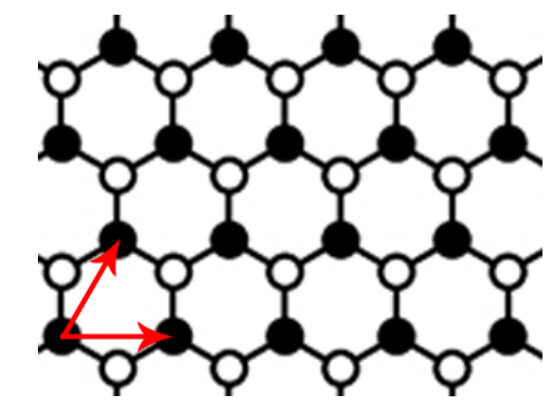

Figure 2.8: Graphene (honeycomb) lattice, where the typical distance between two dots is around 0.1 nanometers. The two colors of the dots denote the two different sublattices. The red arrows note the Bravais vectors.

\subsection{Fundamental properties of graphene}

This section briefly reviews some fundamental properties of graphene that are necessary to obtain a basic understand of the material. These properties are relevant for my thesis in a general sense, but also specifically for chapters 3,6 and 7 that directly treat graphene nanodevices. This overview of this section based on the three review articles by Allain and Fuchs [69], Castro Neto et al. [20] and Goerbig [127]. Since all of the concepts that I will present here are well-established, I will not go into any detail but point to one of the three review papers when appropriate. In addition, to make it easier to connect graphene with the papers included in this dissertation, at the end of each subsection I point out how the knowledge of the subsection is taken advantage of in the papers.

\subsubsection{Energy dispersion of graphene}

Graphene is a two dimensional crystal made exclusively out of carbon atoms arranged in a periodic honeycomb pattern, as seen in Fig. 2.8. This special structure of graphene gives rise to many physical properties that have not been explored before its creation, for the first time in 2004 [13]. A review of the electronic properties of graphene is given in [20], while a review of the transport properties is given in [128].

The honeycomb lattice, in which graphene crystallizes, is not a Bravais lattice but instead it is a triangular Bravais lattice with a two atom basis, or equivalently as two triangular sublattices $A$ and $B$ (white and black dots in Fig. 2.8). The average distance between each carbon atom is $a \approx 0.142 \mathrm{~nm}$. Each triangular sublattice is composed by the two fundamental vectors (Fig. 2.8)

$$
\mathbf{a}_{1}=a \sqrt{3}(1,0), \mathbf{a}_{2}=a \sqrt{3}\left(\frac{1}{2}, \frac{\sqrt{3}}{2}\right) .
$$

Notice that the Bravais lattice constant is not the carbon-carbon distance but $a_{0}=a \sqrt{3} \approx 0.224$ 
nm. The fundamental wavevectors of the reciprocal lattice are

$$
\mathbf{a}_{1}^{*}=\frac{4 \pi}{3 a}\left(\frac{\sqrt{3}}{2},-\frac{1}{2}\right), \mathbf{a}_{2}^{*}=\frac{4 \pi}{3 a}(0,1) .
$$

The reciprocal lattice is also a honeycomb lattice of constant $a_{0}^{*}=4 \pi / 3 \sqrt{3}$. The first Brillouin Zone (BZ) is also a hexagon but rotated $90^{\circ}$ with respect to the direct lattice, as shown in Fig. 2.9. There are two inequivalent corners noted by the $\mathbf{K}^{+}$and $\mathbf{K}^{-}$points (meaning that it is not possible to translate from $\mathbf{K}^{+}$to $\mathbf{K}^{-}$using linear combinations of the reciprocal fundamental vectors)

$$
\mathbf{K}^{+}, \mathbf{K}^{-}=\left( \pm \frac{4 \pi}{3 \sqrt{3} a}, 0\right) \text { or }\left(\mp \frac{2 \pi}{3 \sqrt{3} a}, \frac{2 \pi}{3 a}\right) \text { or }\left(\mp \frac{2 \pi}{3 \sqrt{3} a},-\frac{2 \pi}{3 a}\right) .
$$

All equivalent corners of the first BZ are connected by a transformation by the fundamental vectors of the reciprocal lattice.

The energy dispersion of a material is an equation that gives the energy of an electron wave with respect to its wavevector. This relation is of utmost importance for any material, in the context of condensed matter physics, since all fundamental properties of the material (e.g. electron density) are derived from the dispersion relation. The energy dispersion is typically computed within the tight-binding framework [27]. We will not go through the necessary calculations of the framework that lead to the dispersion relation. Instead I point the interested reader to an excellent introduction by Goerbig [127]. The resulting expression is [127, 20, 128]

$$
\epsilon_{\lambda}(\mathbf{k})=\lambda t \sqrt{3+f(\mathbf{k})}-t^{\prime} f(\mathbf{k})
$$

with

$$
\begin{aligned}
f(\mathbf{k}) & :=2 \sum_{i=1}^{3} \cos \left(\mathbf{k} \cdot \mathbf{a}_{i}\right) \\
& =2 \cos \left(\sqrt{3} k_{x} a\right)+4 \cos \left(\frac{\sqrt{3}}{2} k_{x} a\right) \cos \left(\frac{3}{2} k_{y} a\right)
\end{aligned}
$$

and $t$ is the nearest neighbor hopping constant and $t^{\prime}$ is the effective next-nearest neighbor hopping constant. $\lambda= \pm 1$ is the band index (with +1 for electrons and -1 for holes, see [127]).
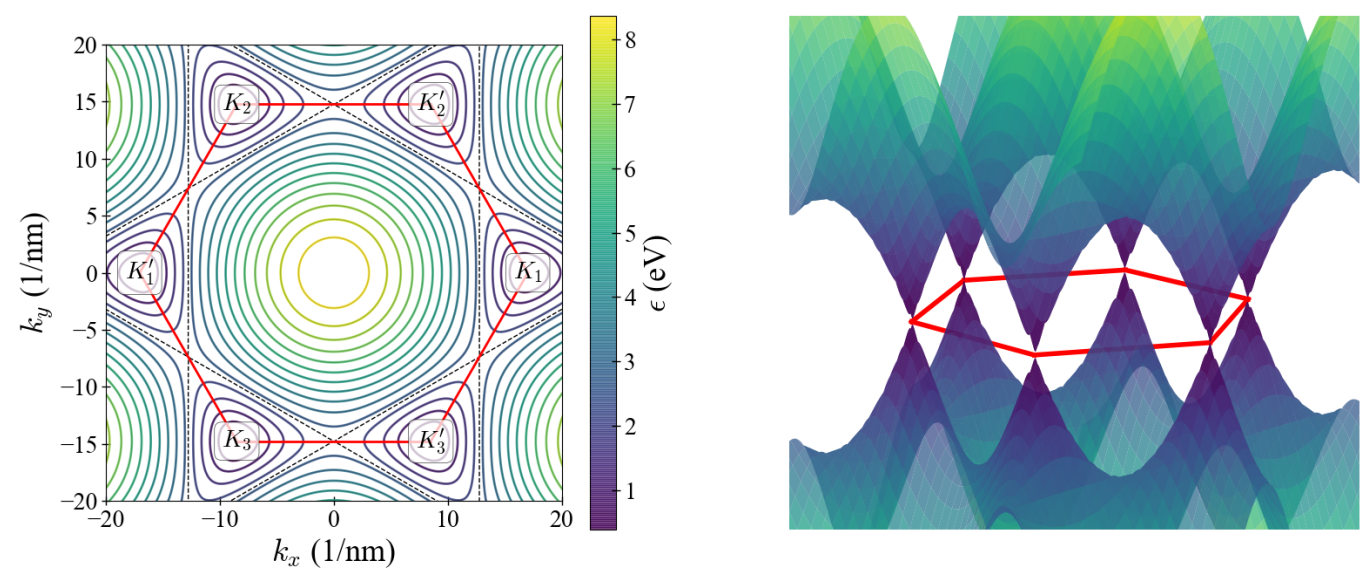

Figure 2.9: Brillouin Zone (BZ) of graphene (area within the red hexagon) and dispersion relation (left: colored contours, right: three-dimensional surface plot), using $t=2.8, t^{\prime}=0 \mathrm{eV}$. The corners of the $B Z$ are noted (the inequivalent corners are $K, K^{\prime}$ with any subscript). The dispersion relation becomes perfect cones when approaching $\epsilon=0$, which is why these points are called "valleys", but here the plotting distorts the structure a bit. 
Fitting (2.3.4) to data from self-consistent density functional theory studies, one finds $t \simeq 2.7 \sim$ $2.8 \mathrm{eV}$ and $t^{\prime}=0.1 t$ [20]. Eq. (2.3.4), which is plotted in Fig. 2.9, is relevant for the third paper of my thesis (chapter 6). This is because there we examined the entirety of the BZ and looked at nearly all energy values. We used the divergence of eq. (2.3.4), to obtain a group velocity value for any wavevector, which is necessary in order to understand the transport direction that electrons move in. The expression one obtains for the group velocity then is

$$
\begin{aligned}
& v_{g, x}=\frac{\sqrt{3} a}{\sqrt{f(k)+3}}\left(-\lambda t+2 t^{\prime} \sqrt{f(k)+3}\right)\left(\sin \left(\frac{\sqrt{3} a}{2} k_{x}\right) \cos \left(\frac{3 a}{2} k_{y}\right)+\sin \left(\sqrt{3} k_{x} a\right)\right) \\
& v_{g, y}=+\frac{3 a \cos \left(\frac{\sqrt{3} a}{2} k_{x}\right)}{\sqrt{f(k)+3}}\left(-\lambda t+2 t^{\prime} \sqrt{f(k)+3}\right) \sin \left(\frac{3 a}{2} k_{y}\right) .
\end{aligned}
$$

The result of eq. (2.3.4) considers only the $p_{z}$ orbitals of each individual carbon atom [127]. Since each carbon atom contributes one electron and each electron may occupy spin up or spin down states, the hole band is completely filled and the electron band is completely empty (at zero temperature). The Fermi level is therefore located at the points where the two bands touch each other, meaning that the Fermi surface (for undoped graphene) consist of just the two (inequivalent) points $\mathbf{K}, \mathbf{K}^{\prime}$ of the Brillouin zone. Notice that for $t^{\prime}=0$, the energy bands are symmetric for the electrons and holes, in the sense that there is only a sign inversion for the dispersion expression.

\subsubsection{Dirac particles in graphene}

For realistic dopings occurring in most experiments, the excitations (chemical potential level) are considered to be at energies very close to the neutrality point (the point where the electron and hole bands meet, for pure graphene this is $\epsilon=0),|\epsilon| \ll t[128]$. Due to the form of the dispersion relation these excitations exist on cones which have the corners of the BZ as centers (see Fig. 2.9), also called Dirac points. It is useful then to conduct a first order approximation around these points, in order to get an effective continuum description. This will simplify the dynamics at small energies and give us a good intuition about how the carriers (electrons/holes) behave.

We first introduce the concept of the valley pseudo-spin and denote it by $\xi= \pm 1 . \xi=1$ indicates the $\mathbf{K}^{+}$valley and $\xi=-1$ the $\mathbf{K}^{-}$valley. We call these points "valleys" because the dispersion surface around them looks like a cone. Let

$$
\mathbf{q}=\mathbf{k}-\mathbf{K}^{\xi}
$$

Then $\mathbf{q}$ is the wavevector relative to the Dirac point so that $|\mathbf{q}| \ll\left|\mathbf{K}^{\xi}\right| \approx 1 / a$. For detailed calculations of the expansion see [127]. The resulting low-energy effective Hamiltonian turns out to be

$$
\hat{H}_{\xi}(\mathbf{q})=\hbar v_{F}\left(\xi q_{x} \hat{\sigma}_{x}+q_{y} \hat{\sigma}_{y}\right)=\hbar v_{F}\left(\begin{array}{cc}
0 & \xi q_{x}-i q_{y} \\
\xi q_{x}+i q_{y} & 0
\end{array}\right)
$$

which satisfies the effective Schrödinger equation $\hat{H}_{\xi} \psi=\epsilon \psi$. We have defined the Fermi velocity as

$$
v_{F}=\frac{3 t a}{2 \hbar} \approx 10^{6} \mathrm{~m} / \mathrm{s} \approx \frac{c}{300}
$$

( $c$ is the speed of light) and $\hat{\sigma}_{x}, \hat{\sigma}_{y}$ denote the two dimensional Pauli matrices

$$
\hat{\sigma}_{x}=\left(\begin{array}{cc}
0 & 1 \\
1 & 0
\end{array}\right), \quad \hat{\sigma}_{y}=\left(\begin{array}{cc}
0 & -i \\
i & 0
\end{array}\right) .
$$


The energy dispersion of this Hamiltonian is easily found to be

$$
\epsilon_{\lambda}(\mathbf{q} ; \xi)=\lambda \hbar v_{F}|\mathbf{q}| \quad \text { with } \quad \lambda= \pm 1
$$

which is independent of $\xi$ meaning that we have a two-fold valley degeneracy. Also, it is obvious that the velocity of the low energy quasi-particles does not increase linearly with $k$ as is commonly the case with many other $2 \mathrm{D}$ electron systems. Here the velocity is a constant, given by the Fermi velocity

$$
\mathbf{v}_{g}=\frac{1}{\hbar} \nabla_{\mathbf{q}} \epsilon=\lambda v_{F} \frac{\mathbf{q}}{|\mathbf{q}|},
$$

hinting that the carriers behave as photon-like particles. This also shows that the valence band $(\lambda=-1)$ has group velocity opposite to its momentum.

\section{Massless Relativistic Quasiparticles of 2+1 Dimensionality}

The Hamiltonian of the form $\hat{H} \sim \hat{\boldsymbol{\sigma}} \cdot \mathbf{q}$ is called the Dirac Hamiltonian and describes massless relativistic fermions in two-dimensional space. Because the Hamiltonian is already a matrix, the wavefunction is a spinor. In the original case studied by Dirac the "spin" corresponds to the real electron spin. In the case of graphene the spin is called "sub-lattice pseudospin" because it instead serves as an index of being in sublattice $A$ or $B$. This sub-lattice pseudospin is a conserved quantity for free particles. The Dirac Hamiltonian is valid in graphene as long as the energies considered are well below the hopping amplitude $|\epsilon| \ll t$ (typically up to $\pm 0.2 \mathrm{eV}$ ).

However, there are two such Dirac cones where the quasi-particles behave as massless relativistic fermions. Although the system is two dimensional in space, the inclusion of both cones in the effective Dirac Hamiltonian makes $\hat{H}$ a $4 \times 4$ matrix and the wavefunction a 4 -component spinor. This is the same case with the 3D Dirac Hamiltonian for "true" Dirac fermions, due to the two projections of spin and two values of the charge degree of freedom [129]. The small energy quasi-particles of graphene are therefore described by what is called " $2+1$ "-dimensional quantum electrodynamics [130].

This low-order approximation of graphene is used in the third paper in several points. Firstly, it allows for analytical treatment of Klein Tunneling (see sec. 2.3.6). Secondly, it defines the concepts of intra- and inter-valley scattering. If an electron wave with momentum in one valley undergoes a (momentum-changing) process that results in the electron being in the other valley after scattering, then we have intervalley scattering. Otherwise the processes is intravalley scattering.

\subsubsection{Single Valley Approximation}

In general in nanodevices, non-constant space depending electrostatic potentials exist $V=V(\mathbf{r})$ (and maybe constant in parts). It is important then to consider under what approximations, does the single Dirac cone Hamiltonian represent the graphene system accurately. Let us call $w$ the characteristic length scale over which the potential varies. In order for inter-valley scattering to be avoided, the potential must be smooth on the lattice scale i.e. $w \gg a_{0} \approx 0.2 \mathrm{~nm}$. This is an important requirement because the distance between the valleys in reciprocal space is $\left|\mathbf{K}-\mathbf{K}^{\prime}\right| \approx 1 / a_{0}$ and the Fourier transform of the aforementioned potential $\tilde{V}(\mathbf{q})$ will be nonzero for $q \leq 1 / w \ll 1 / a_{0}[127]$.

For such potentials the valleys are decoupled and electrons/holes in graphene can be described by a single valley 2D massless Dirac Hamiltonian $\hat{H}=\hat{H}_{k i n}+\hat{V}(x, y)$ [131]. In addition, since the potential varies slowly over the distance between two neighboring atoms ( $A$ and $B$ sublattice) the potential matrix $\hat{V}$ can be taken to be diagonal in the sublattice space, $\hat{V}(x, y)=V(x, y) \hat{I}$. We will refer to these approximations as the single valley approximation. Importantly, the sublattice pseudospin is a conserved quantity within this approximation, since the potential in this case (by definition) cannot scatter in sublattice space [69]. 
The single valley approximation is used throughout the first paper in chapter 3 . There we approximated the electron kinetic energy using the simplest possible dispersion form, $\epsilon=v_{F} \hbar|\mathbf{k}|$.

\subsubsection{Cyclotron radius \& electron density}

Due to the connection between momentum and energy for the first order approximation of graphene, the cyclotron radius scales differently with energy than what would be the case of a standard semiconductor, since

$$
R_{c}:=\frac{p}{e B}=\frac{E}{v_{F} e B} \Rightarrow R[\mathrm{~nm}]=10^{3} \frac{E[\mathrm{eV}]}{B[\mathrm{~T}]} .
$$

The electron density, immediately obtained from the dispersion relation, is also very different for graphene. To compute the density one first needs the density of states, which for graphene is linear within the Dirac approximation [128]

$$
\rho(\epsilon)=\frac{g_{v} g_{s}}{2 \pi} \frac{|\epsilon|}{\left(\hbar v_{F}\right)^{2}} .
$$

Here $g_{s}=2$ is the spin degeneracy for the case of free electrons and $g_{v}=2$ is the valley degeneracy, assuming the single cone approximation. The electron density $n_{s}$ is simply the integral of $\rho$ up to the Fermi energy (assuming very low temperatures)

$$
n_{s}=\int_{0}^{E_{F}} \rho(\epsilon) d \epsilon=\frac{1}{\pi} \frac{E_{F}^{2}}{\left(\hbar v_{F}\right)^{2}} .
$$

From this one can obtain the Fermi wavevector from the electron density, and vice versa, using $k_{F}=\sqrt{\pi n_{s}}$.

Both $R_{c}$ and $n_{s}$ are crucial values for the first manuscript of chapter 3 . The experimental measurements only provide an estimate for $n_{s}$ which we then transformed into the Fermi wavevector. We then used this wavevector to deduce the correct cyclotron radius to be used in both the continuous system and the billiard system considered in chapter 3 .

\subsubsection{Beyond the Dirac Regime}

Even though the continuum description can be based just on the first order, the second order approximation provides a lot of insight and leads to new effects and thus it is worth studying. The energy dispersion calculated up to second order is given by [20, 127]

$$
\epsilon_{\lambda}(\mathbf{q} ; \xi)=3 t^{\prime}+\lambda \hbar v_{F}|\mathbf{q}|-|\mathbf{q}|^{2}\left[\frac{9 t^{\prime} a^{2}}{4}+(\lambda \cdot \xi) \frac{3 t a^{2}}{8} \cos \left(3 \varphi_{\mathbf{q}}\right)\right]
$$

with

$$
\varphi_{\mathbf{q}}=\arctan \left(q_{y} / q_{x}\right)
$$

Using the definition $v_{F}=\frac{3 t a}{2 \hbar}$, one can reduces the above to

$$
\epsilon_{\lambda}(\mathbf{q} ; \xi)=3 t^{\prime}+\lambda \hbar v_{F}|\mathbf{q}|+\left(\hbar v_{F}|\mathbf{q}|\right)^{2} \frac{1}{t}\left[\left(\frac{t^{\prime}}{t}\right)-\frac{\lambda \xi}{6} \cos \left(3 \phi_{\mathbf{q}}\right)\right] .
$$

Second order corrections transform the Fermi surfaces from circles to smoothened triangles as the energy increases, adding an anisotropic group velocity. The reason for this is the tripling of the period, due to the term $\cos (3 \phi)$, which reflects the symmetry of the underlying lattice and is also referred to as trigonal warping [127]. This term is in addition coupled with the valley pseudo-spin which means that the energy dispersion is anisotropic in $\mathbf{q}$ with a different orientation for the different valleys. The second order approximation is accurate up to energies of $2 t / 3 \approx 2 \mathrm{eV}[127]$. 
Group Velocity We have seen the group velocity for the first order approximation in eq. (2.3.12). For the second order dispersion, the group velocity formally defined as $\mathbf{v}_{g}:=\nabla_{\mathbf{q}} E / \hbar$ can be obtained from eq. (2.3.15). The result is

$$
\begin{aligned}
& v_{x}=-\frac{\hbar \lambda v_{F}^{2} \xi}{3 t} q_{x} \cos (3 \phi)+\frac{2 \hbar}{t^{2}} q_{x} t^{\prime} v_{F}^{2}-\frac{\hbar \lambda v_{F}^{2} \xi}{2 t} q_{y} \sin (3 \phi)+\lambda v_{F} \cos (\phi) \\
& v_{y}=\frac{\hbar \lambda v_{F}^{2} \xi}{2 t} q_{x} \sin (3 \phi)-\frac{\hbar \lambda v_{F}^{2} \xi}{3 t} q_{y} \cos (3 \phi)+\frac{2 \hbar}{t^{2}} q_{y} t^{\prime} v_{F}^{2}+\lambda v_{F} \sin (\phi) .
\end{aligned}
$$

Based on the above equations, we can map each pair $\left(q_{x}, q_{y}\right)$ to an energy-propagation angle pair $(E, \theta)$ (where $\theta=\arctan \left(\frac{v_{y}}{v_{x}}\right)$ ) and vice versa. Inversion for each valley is possible, however it has to be done numerically as there is no way to invert $\theta$ for $\phi$ analytically.

This second order approximation shown here is used in the third manuscript (chapter 6), specifically in section II.D where we inquire about the nature of Klein tunneling in the presence of trigonal warping.

\subsubsection{Klein Tunneling}

Particles satisfying the Dirac equation have some very peculiar properties when one considers typical tunneling scenarios, like e.g. incidence on a potential barrier. What was first discovered by Oscar Klein in 1929 [72] is that under certain conditions Dirac particles can penetrate a potential barrier with perfect transmission, under some conditions. What was astonishing is that this transmission remains perfect even in the case of infinitely wide and high barriers. This section is a brief overview of the phenomenon as it is applied in the case of graphene, by following the pedagogical introduction by Allain and Fuchs [69]. Keep in mind that the eigenstates of the Dirac Hamiltonian $H=\lambda \hbar v_{F} \sigma \cdot \mathbf{k}$ are given (in position representation) by [69, 129]

$$
\psi_{\lambda}^{\xi}(\mathbf{r})=\frac{e^{i \mathbf{q} \cdot \mathbf{r}}}{\sqrt{2}}\left(\begin{array}{c}
1 \\
\lambda e^{+i \xi \phi_{\mathbf{q}}}
\end{array}\right)
$$

with $\phi_{\mathbf{q}}=\arctan \left(q_{y} / q_{x}\right)$. Notice also that the wavenumber $\mathbf{q}$ is measured with the respect to the Dirac points $\mathbf{K}^{\xi}$. Depending on the context, $\phi$ is frequently called the "angle of incidence" because in this Dirac approximation the group velocity direction coincides with the wavevector direction. The entirety of this section assumes the single valley approximation 2.3.3.

A tunneling event in the context of the single valley approximation works as follows. An electron (present in the electron band) is incident on a potential step, like in Fig. 2.10(a). After the step, the electron is transmitted (with some probability $T$ ) into the hole band. Transmission with non-zero probability in this case is always possible, because there always exist states in the hole band that match the required energy, momentum and sublattice pseudospin due to the symmetry of the Dirac dispersion (see sketch). Notice that matching the pseudospin is necessary because by definition in this context the sublattice pseudospin is a conserved quantity.

It turns out that for Dirac particles having angle of incidence $\phi=0$, reflection from the potential barrier is only possible by reversing the sublattice pseudospin (see [69] for a proof). This is impossible however, since by definition this potential step cannot change the sublattice pseudospin, which is a conserved quantity. Therefore, the only possible case for $\phi=0$ is that no reflection happens whatsoever, i.e. perfect transmission occurs $T(\phi=0)=1$. This must happen regardless of the details of the potential, assuming that we remain in the single valley approximation. The manifestation of Klein tunneling in graphene comes from this fact, which analytically expresses itself in the transmission probability formula $T(\phi)$, which always satisfies the property $T(0)=1$. 


\section{Klein Tunneling in Steps}

Sec. 2.3.3 defined what properties the characteristic length scale of a potential must satisfy in order for inter-valley scattering to be averted, i.e. $w \gg a_{0}$. In addition, the potential can be smooth $\left(w \gg 1 / k_{F}\right)$ or $\operatorname{sharp}\left(w \ll 1 / k_{F}\right)$ on the Fermi wavelength scale $k_{F}=\left|E_{k i n}\right| / \hbar v_{F}$. If it is sharp, we can assume that it is piecewise constant (step or square barrier). If however this condition does not apply, one has to use smooth formulas for the potential function, which are presented in the next section.

An electron is in the presence of a potential $V(x)$ such that: $V(x<0)=-V_{0} / 2$ and $V(x>0)=V_{0} / 2$. Let $\phi_{i}$ be the incidence angle of the wave coming from the left and with energy $E$ so that $E-V_{0} / 2$ is negative after the step (so the electron moves into the valence band after transmission). Let $\phi_{r}$ and $\phi_{t}$ be the angles of reflection and transmission. Due to the conservation of energy and $y$ momentum, we have that

$$
\hbar v_{F} q_{y, j}=\lambda E_{\text {kin }, j} \sin \phi_{j}=\text { const. } \quad \forall j
$$

with $j=i, r, t$. In the case of transmission $\lambda$ and $E_{k i n}$ change sign. The above directly gives $\phi_{t}$ through

$$
\left(E+\frac{V_{0}}{2}\right) \sin \phi_{i}=-\left(E-\frac{V_{0}}{2}\right) \sin \phi_{t}
$$

To find the transmission probability one has to match the wavefunctions at $x=0$ (see [69]), with result

$$
T\left(\phi_{i}\right)=-\frac{\cos \phi_{i} \cos \phi_{t}}{\sin ^{2}\left(\frac{\phi_{i}+\phi_{t}}{2}\right)} .
$$

For $E=0$ one has the simplification of $T=\cos \left(\phi_{i}\right)^{2}=1-\left(q_{y} / q_{F}\right)^{2} . T$ is defined only up to the so-called critical angle, and vanishes otherwise. This angle is defined by

$$
\phi_{c}=\arcsin \left(\frac{V_{0} / 2-E}{V_{0} / 2+E}\right)
$$

but only makes sense if $E>0$. When this condition is satisfied, any transmission for $\phi_{i}>\phi_{c}$ is 0 (the transmitted wavefunction is evanescent) and total internal reflection takes place. What stands out is that $T(\phi=0)=1$ independently of the characteristics of the potential. This is a manifestation of Klein tunneling, and it is related with the absence of backscattering (discussed in detail in [69]).
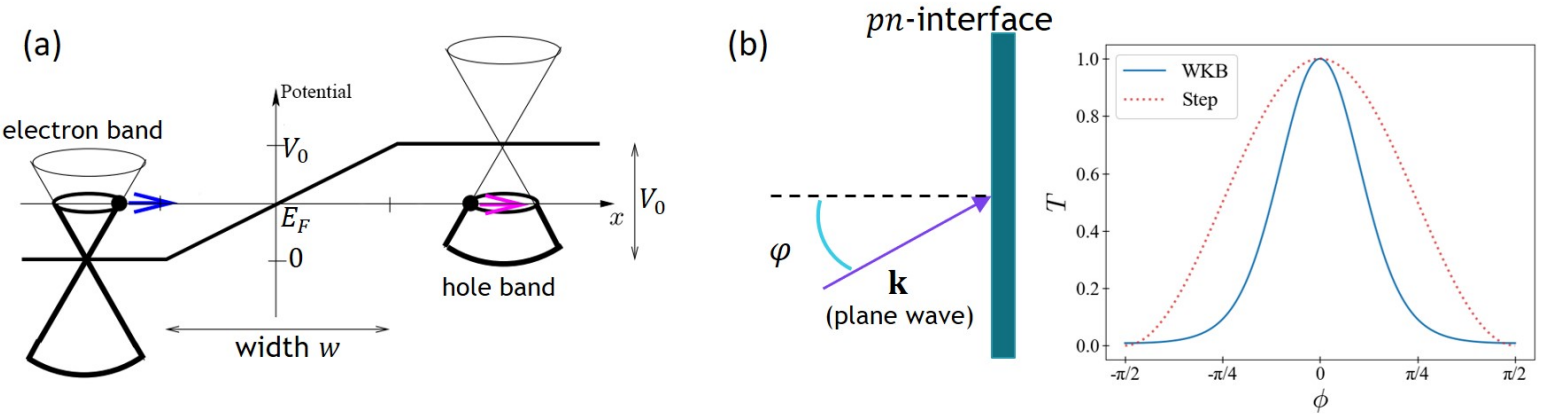

Figure 2.10: (a) An electron incoming from the left is incident on a potential step (black line). It is then transmitted into the hole band via reversing its band index and momentum, but conserving its sublattice pseudospin (the pseudospin in this simple sketch is simply either positive or negative slope of the cone). (b) Klein tunneling: the transmission probability decays exponentially with the angle of incidence. Step refers to eq. (2.3.21) while WKB to eq. (2.3.26). 


\section{Klein Tunneling in Smooth Junctions}

We will now consider the case of tunneling through a smooth junction, $\lambda_{F}=1 / k_{F} \ll w$. This was first done by Cheianov and Fal'ko in 2006 [70], using the WKB approximation, and further summarized in [69]. Let's assume that the smooth junction has a total width of $w$ and total potential difference of $V_{0}$. It can be approximated by either a linear

$$
V_{l}(x)= \begin{cases}V_{0} \frac{x}{w} & \text { for }|x|<w / 2 \\ \operatorname{sign}(x) \frac{V_{0}}{2} & \text { else }\end{cases}
$$

or hyperbolic function

$$
V_{h}(x)=\tanh \left(\frac{x}{w / 2}\right) .
$$

We assume an electron approaching from the left and also assume Fermi energy $E=E_{F}=0$. Of course, this means that the kinetic energy is

$$
E_{k}=\hbar v_{F} \sqrt{k_{x, 0}^{2}+k_{y}^{2}}=v_{F} \sqrt{p_{x, 0}^{2}+p_{y}^{2}}=E-V(-\infty)=V_{0} / 2
$$

with Fermi wavenumber $k_{F}=V_{0} /\left(2 \hbar v_{F}\right)$. Let the incidence angle be $\phi, p_{x, 0}=\hbar k_{F} \cos \phi$. After the junction, the electron is expected to emerge in the far-right side of the junction with $y$ momentum conserved $p_{y}^{\prime}=p_{y}=\hbar k_{F} \sin \phi$ and $x$-momentum reversed $p_{x}^{\prime}=-p_{x, 0}$, with some probability $T(\phi)$, so that the pseudospin is conserved. In [70] the authors are able to find this probability as

$$
T(\phi)=e^{-\pi k_{F} \frac{w}{2} \sin ^{2} \phi} .
$$

The main result of [70] is that the above formula is exact for any smooth junction with "width" $w$ (and symmetric around 0 energy), as long as the incidence angles are not too close to $\pi / 2$, where the transmission in fact vanishes. Once again we see that $T(\phi=0)=1$ independent of the potential parameters and incoming energy, which again reflects Klein tunneling.

The smooth case has differences from the step case; in the former a collimation effect takes place. The junction focuses the electronic flow by allowing the transmission of only the trajectories that are close to normal incidence. This is true for angles $|\phi| \leqslant 1 / \sqrt{\pi k_{F} w}$. The non-symmetric case of $E_{k} \neq V_{0} / 2$ has been considered in [132]

$$
T(\phi) \approx \exp \left(-\pi \frac{2 k_{1}^{2}}{k_{1}+k_{2}} \frac{w}{2} \sin ^{2} \phi\right),
$$

with $k_{1}, k_{2}$ the Fermi wavevectors in the left and right zones $\left(k=E_{k i n} / \hbar v_{F}\right)$. Once again, in all cases shown here, we see Klein tunneling: $T(0)=1$ independently of the parameters of the potential barrier meaning always perfect electron transmission at normal incidence.

\section{Impact on transport}

The potential impact of Klein tunneling on transport was first discussed by Katsnelson, Geim and Novoselov in [71]. A first consequence regards disorder-induced localization. In a typical parabolic dispersion semiconductor and at small excitation levels, disorder will create random potential barriers that will confine carriers in arbitrary configurations [71]. In graphene however the effect changes, since all barriers will be at some point penetrated, since the random electron velocities will come close to angle of incidence $\phi=0$ for an arbitrary barrier. Another consequence regards making transistors out of graphene. Since it is not simple to turn on a potential barrier and stop current flow due to Klein tunneling, the working principle of the transistor does not straight-forwardly apply to graphene and different approaches are necessary. For a more in-depth review of transport in graphene please see [128]. 
This entire section on Klein tunneling is of crucial importance for the third paper in chapter 6. The paper analyzes Klein tunneling in various contexts: a simple pn-junction injected with Dirac eigenstates, a complicated wavefunction resulting via boundary-induced scattering and finally in energies beyond the Dirac regime.

\subsubsection{Lead Modes}

For current to flow through a nanodevice, it has to be connected to leads (wires) which, in a loose sense, bring electron waves in and out of the device. These leads are typically assumed to be of the same material as the nanodevice, here graphene, and are therefore represented as systems with finite width and (semi-)infinite length. Also called graphene nanoribbons (GNR), they have been studied analytically in the literature $[133,134,135]$. This section reviews some core results that are fundamentally relevant for the third paper, chapter 6 , which considers nanodevices connected to such GNRs.

As was discussed in sec. 2.3.1, the energy dispersion is necessary to deduce various crucial electronic properties of a material. Here, since the GNR are periodically extending only in one spatial dimension, the energy dispersion is a function of only a single wavenumber (the one of the longitudinal direction). The system then is composed of a unit cell which is repeated indefinitely along the period direction. In Fig. 2.11 I show a "zigzag" graphene nanoribbon (ZGNR), which will be the model system for the leads, along with its unit cell. The zigzag edge (top of Fig. 2.11) has the interesting property that the atoms of each side belong to the same sublattice.

Using Kwant [29] (see sec. 2.4), a software for quantum transport within the tight-binding framework, one can compute the energy bands of the ZGNR. The results of numeric calculations based on the unit cells defined in Fig. 2.11 are presented in Fig. 2.12. It should be noted that there are multiple bands in the dispersion; this is because the unit cells of the GNRs are composed of multiple carbon atoms. The number of bands equals the number of atoms in the unit cell. Besides "bands" the different dispersion curves are also called "modes" (because they correspond to transverse oscillation modes of the wavefunction).

Due to the property of the terminating edges, the ZGNR retain the two inequivalent valleys of the normal two dimensional graphene case, as can be seen in Fig. 2.12. The Dirac valleys now live in $k_{x} a_{0}= \pm 2 \pi / 3$ (the bands are symmetric over translations by $\pi$ ). The ZGNR are always metallic, due to the existence of bands for any energy level. Let us now review from [134] the functional form of the wavefunctions in a ZGNR, given the single valley approximation.

The wavefunction in the continuum description of a ZGNR is a spinor of the form

$$
\mathcal{W}(\mathbf{r})=\exp \left(q_{x}\right)\left(\begin{array}{c}
\Phi_{A}(y) \\
\Phi_{B}(y)
\end{array}\right)
$$

with $\Phi$ being the transverse wavefunction in each sublattice, $q_{x}$ the longitudinal wavenumber as measured from the Dirac point. Here I only focus on one of the two Dirac valleys, but I will

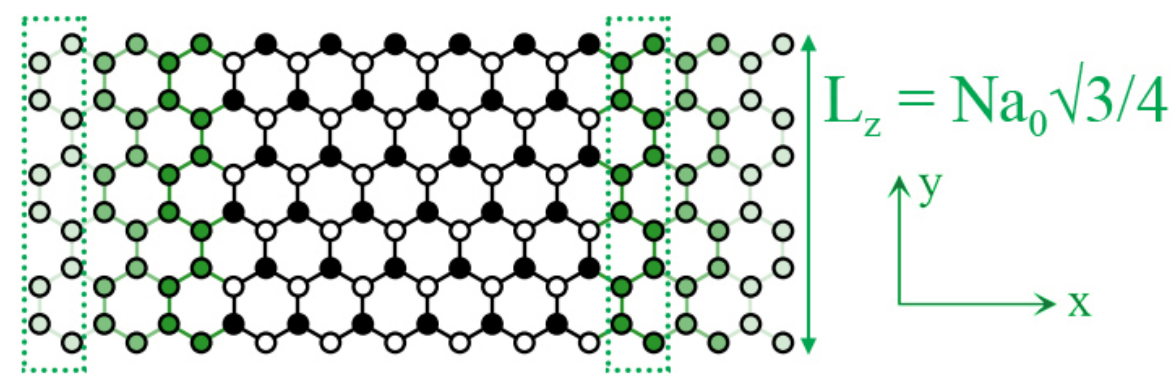

Figure 2.11: Different terminations of a graphene nanoribbon. Top, green: zigziag, bottom, blue: armchair. The dotted rectangles indicate the unit cells. Next to each nanoribbon I show the dependence of the width $L$ with respect to the number of atoms in the cell $N$. Figure produced using Kwant [29]. 
not discuss further any differences between the two cases, because all subsequent properties are transformed from one valley to the other via a reflection $x \rightarrow-x$.

In [134] the authors are able to analytically find the form of $\Phi$, which is

$$
\begin{aligned}
\Phi_{A} & =\sin \left(k_{n} y\right) \\
\Phi_{B} & =\frac{i}{\epsilon}\left[-k_{n} \cos \left(k_{n} y\right)+q_{x} \sin \left(k_{n} y\right)\right] .
\end{aligned}
$$

with $k_{n}$ being the transverse (and discrete) wavenumber and $\epsilon$ the energy of the mode, $\epsilon=$ $\pm \sqrt{q_{x}^{2}+k_{n}^{2}}$ (the \pm here denotes the electron or hole regime). The values of $k_{n}$ can be obtained from $q_{x}$ by solving the nonlinear equation

$$
q_{x}=\frac{k_{n}}{\tan \left(k_{n} L\right)} .
$$

These solutions correspond to confined modes, i.e. standing waves. Due to symmetry, for each solution $+k_{n}$ there is also a solution $-k_{n}$. Besides confined modes there are also other solutions, but not of interest for this thesis, see [134]. Using (2.3.31) the second equation for $\Phi_{B}$ can be simplified greatly to

$$
\Phi_{B}=\mp i \sin \left(k_{n}(L-y)\right) .
$$

These formulas show that the transverse modes, for small energies and wide enough nanoribbons, can be approximated as simple sine modes. This is also shown in the third paper of my thesis, where I use this simple fact to confirm the validity of the Husimi function (which very clearly displays the symmetry of $\pm k_{n}$, as seen in e.g. sec. IIA of the third paper).

\subsection{Why quantum?}

Sec. 2.1.4 (and generally the first two papers of this dissertation, chapters 3 and 5) explains magnetotransport phenomena in graphene-based nanostructures, namely antidot superlattices, using classical mechanics, without any special care for quantum-exclusive phenomena like tunneling. This approach seems to work very well for the devices of Refs. [41, 62], which both use electron beam lithography to create the antidot superlattice. In this scenario the antidots are absolutely inaccessible repellers with respect to the electron motion, which is qualitatively the same case as in GaAs-based antidots, described in sec. 2.1.1. In the same section another technique for fabricating antidots superlattices was mentioned, namely by electrostatic modulations. In the GaAs-based systems, this once again results in inaccessible repellers.
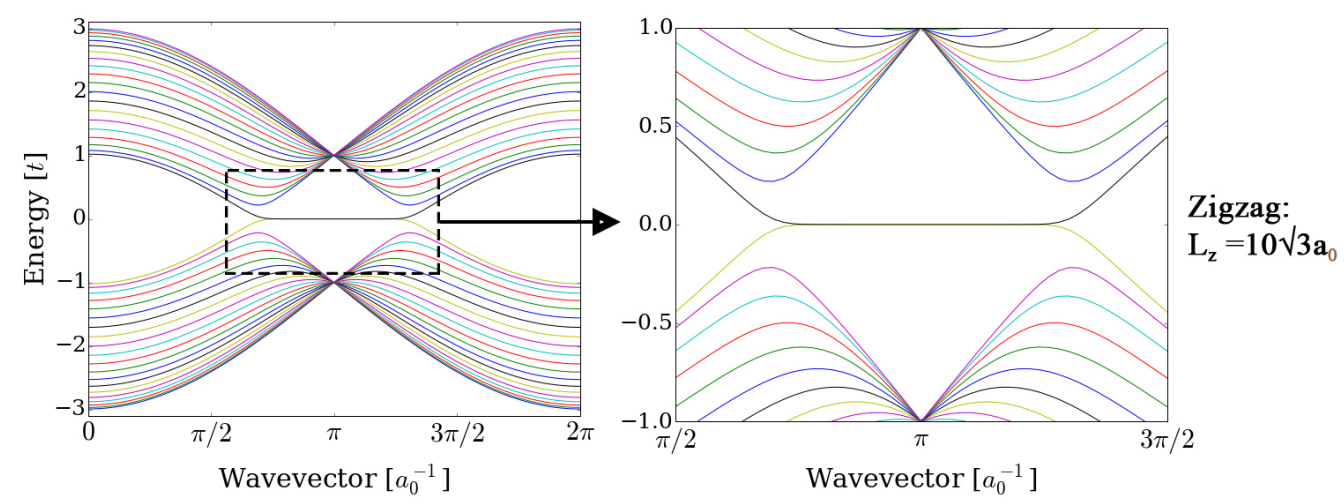

Figure 2.12: Energy bands for different graphene nanoribbons, in units of the hopping parameter t, as they are calculated through tight-binding models. For zigzag the lattice constant $a_{0}$ is the same as graphene's. For the armchair the lattice constant is further multiplied with $\sqrt{3}$. Figure produced using Kwant [29]. 
But one has to wonder, would the same be true for graphene? If an antidot superlattice was made in graphene using electrostatic modulations, then the situation would not the same as with the original GaAs-based devices. Here, the antidots are no longer absolutely inaccessible repellers due to Klein tunneling. If an electron is incident normally on the antidot potential (assuming the potential satisfies the requirements mentioned in sec. 2.3.3), then Klein tunneling dictates that the electron should pass through the antidot, a case that was not possible so far. Fortunately, this scenario has been already realized in experiments. The experimental group from Regensburg that performed the original graphene antidot superlattice experiments has made the same system using electrostatic modulation, as shown in Fig. 2.13(a, b). More details on this device can be found in [136], chapter 9. These new experiments did not display the commensurability peaks with the same clarity as the ones mentioned in sec. 2.1 and chapter 3 . This could be perceived as indication that Klein tunneling should be taken into account when modeling these new devices.

The simplest approach to do this is to continue using classical mechanics (e.g. billiards) but to incorporate the tunneling process into the classical system. This is done using the approach of ray-splitting billiards [63, 64,65]. Here, the standard billiard system is modified so that the particle may penetrate an obstacle upon collision, instead of always being reflected. The penetration event happens with a given probability $T$. In our case, this probability is simply the probability that Klein tunneling occurs (see e.g. eq. (2.3.26)). A ray-splitting periodic Sinai billiard is presented in Fig. 2.13(d). Unfortunately, preliminary results showed that the situation is not that simple and the magnetoresistance only changes slightly when one incorporates Klein tunneling into a billiard, as seen in Fig. 2.13(c).

At the point in time of doing these ray-splitting simulations, the literature on transport in graphene simply had too little content that connected quantum phenomena with semiclassics, which seems to be necessary for devices like e.g. antidot superlattices, which display profound

(a)

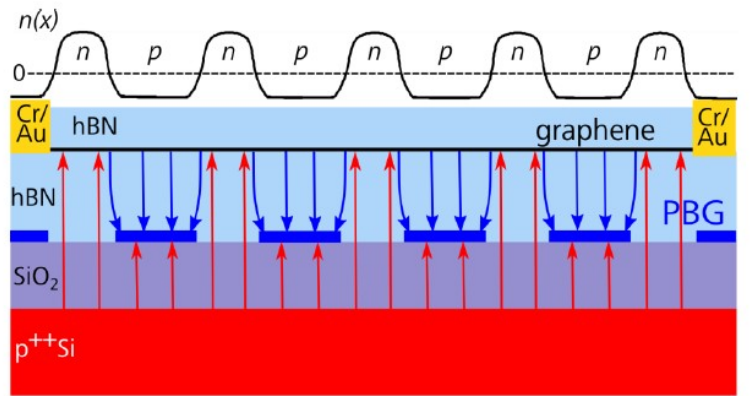

(b)

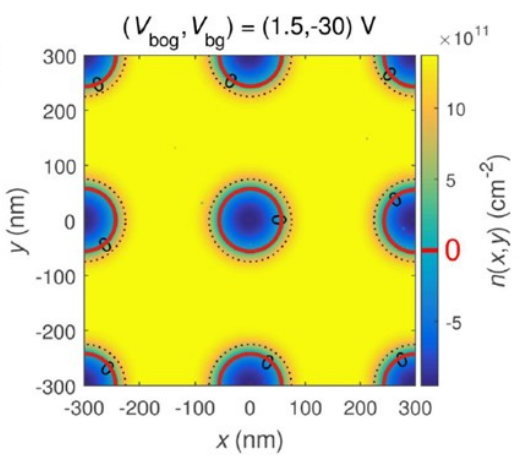

(c)

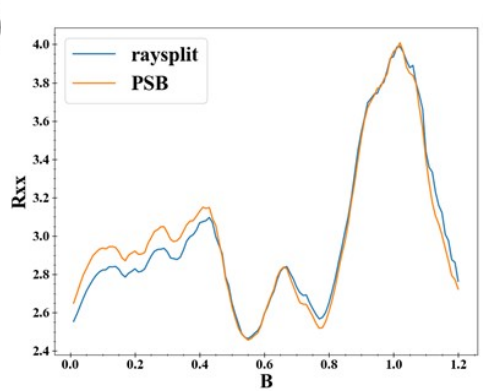

(d)

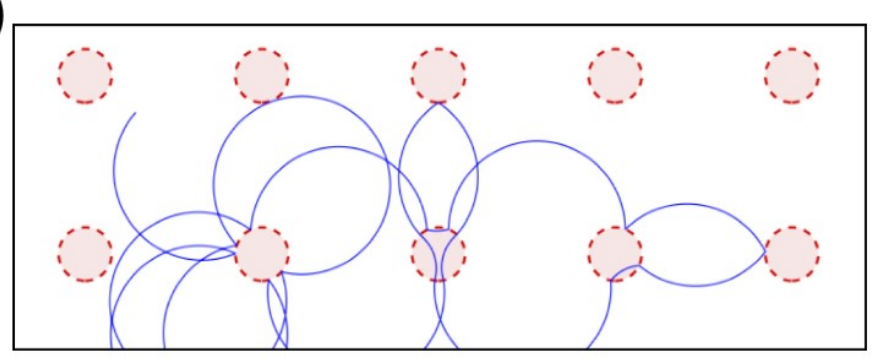

Figure 2.13: ( $a, b)$ New graphene antidot superlattice device using electrostatic modulation. (a) Device setup (with $n(x)$ the electron density variation) and $(b)$ electron density variation throughout the device. The electrostatic potential is directly proportional to the electron density $n(x, y)$. (c) comparison of the magnetoresistance simulations from the periodic Sinai billiard (with "raysplit") and without ("PSB") raysplitting. The motion of the particle in the raysplitting case is shown in (d). (a,b) are kindly shared by Andreas Sandner. 
classical phenomena. To improve this, in the third paper of this dissertation in chapter 6 we studied exemplary nanodevices from a semiclassical perspective, connecting quantum phenomena (e.g. Klein tunneling) with the phase space, providing semiclassical intuition. We did this by first simulating quantum systems, as described in sec. 2.5, and then analyzing them using the Husimi function (defined in sec. 2.6), which is a tool that allows one to obtain semiclassical information from a quantum wavefunction. Note that as this new project was underway, we believe that we have understood why the new experimental devices did not display commensurability peaks with clarity; the real mean free path in the devices was probably smaller than the mean collision time $\kappa$, which according to our analysis, would make the commensurability peaks unobservable in experiments. For this reason in the rest of the thesis there will be no further mention of this new device.

\subsection{Transport simulations and scattering wavefunctions}

This section describes the basics of performing quantum transport simulations using the LandauerBüttiker formalism [66]. This is a well-established approach which is described in more detail in [8] and only summarized here (and also sketched in Fig. 2.14). In the Landauer-Büttiker approach one studies transport through a device, which is referred to as the "scattering region". In this thesis the device is represented as a tight-binding system (i.e. discrete lattice with connections between the lattice sites). This device is connected via semi-infinite leads to "reservoirs" of a specified chemical potential (Fermi energy). The leads in the case of my thesis are graphene nanoribbons (GNR), discussed in detail in sec. 2.3.7. A GRN may have several modes (i.e. wavefunctions) that it can accommodate at a given Fermi energy. These modes are then the electronic wavefunctions that each lead can bring into the scattering region.

While in the device, the wavefunction gets scattered, not only due to the device's geometry, but also due to the presence of scalar or vector potentials. This results in the scattering

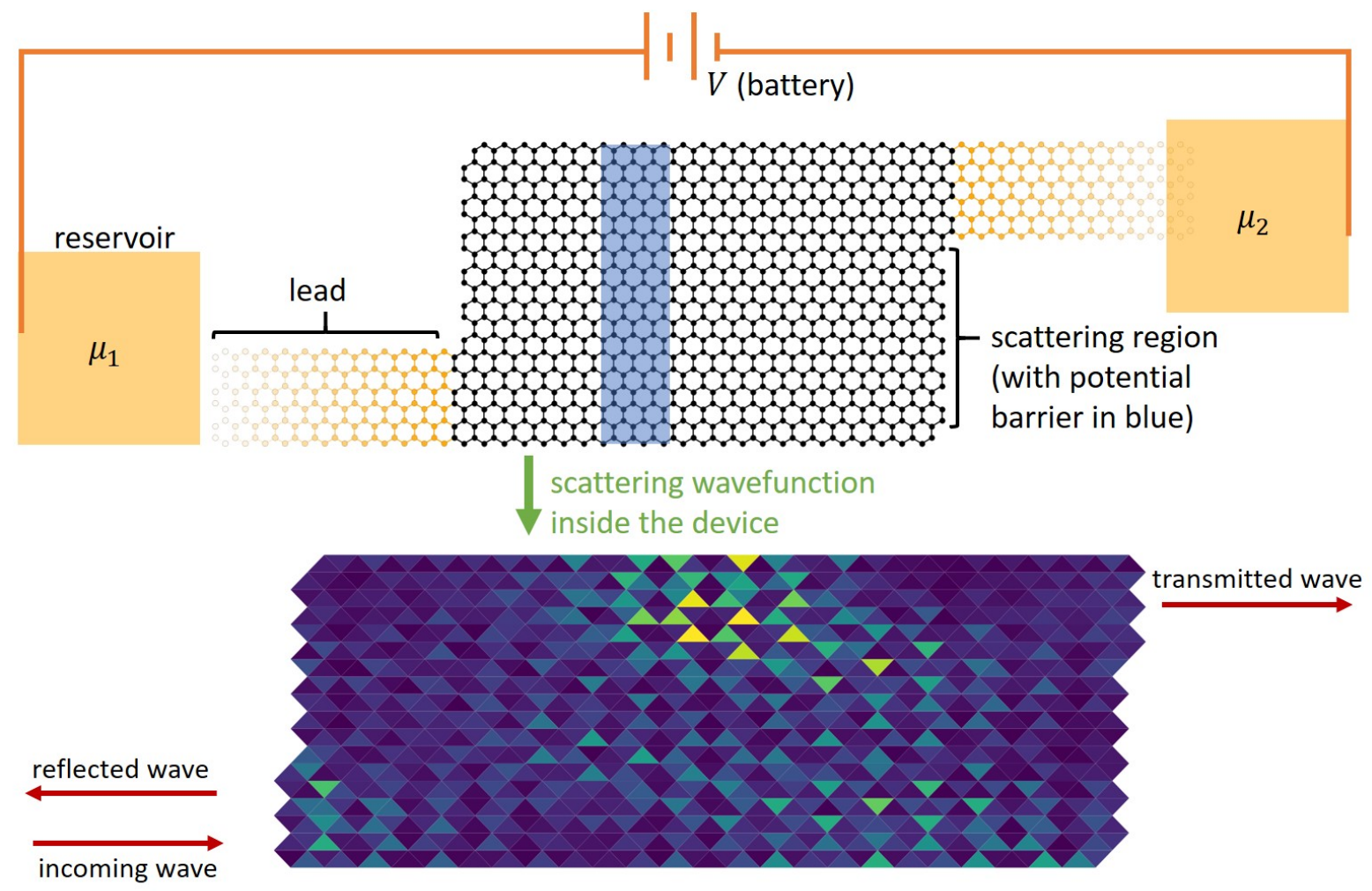

Figure 2.14: A sketch explaining the Landauer-Büttiker formalism. 
wavefunction $\psi_{m}$, each one corresponding to an incoming mode $m$. In my thesis I performed all these quantum simulations with the existing excellent software Kwant [29]. The recursive Green's function method is used to obtain the scattering wavefunctions, and more details can be found in Refs. [8, 29].

After scattering occurs the wavefunction exits the device either through the same lead it entered (reflection) or through another lead (transmission). In the case of only two leads being connected to the device (which is what I studied), the numbers $R_{m}, T_{m}$ quantify the reflection and transmission probability of an incoming electron wave with mode number $m$ (obviously $R_{m}+$ $\left.T_{m}=1 \forall m\right)$. Within this framework the conductance $G$ of the device is directly proportional to the sum of the transmission probabilities, $G \sim \sum_{m} T_{m}[8]$.

Klein tunneling, which is discussed in sec. 2.3.6, crucially depends on the so-called "angle of incidence" $\phi$. Now, as one can imagine, and also by looking at the sketch of Fig. 2.14, the scattering wavefunctions are complicated objects. They are not easily described by a plane wave with a simple angle of incidence $\phi$. So the question is, how can one recover some kind of angle of incidence information from the complicated scattering wavefunctions? To resolve this problem one has to transform the wavefunction to a distribution over wavevectors and positions, so that one can have an estimate of which wavevectors (and thus angles of incidence) are relevant at which positions inside the nanodevice. I describe how to achieve such a feat in the following section.

\subsection{Quantum mechanics and the phase space}

Soon after the first formulation of quantum mechanics, there have been efforts to connect wavefunctions and distributions on the phase space (the joint space of momentum and position) [73]. Such a connection is important for the concept of quantum-classical correspondence, which was discussed in the introduction (chapter 1). All these efforts aim to formally define a so-called quasi-probability distribution on the phase space of classical conjugate variables $x$ and $p$. Here the wording "quasi-probability" is crucial. A true probability distribution cannot be defined for the quantum world, due to the uncertainty principle. Any attempt to define such will always necessarily drop support of Kolmogorov's third axiom of probability, because any phase space point is indistinguishable from any neighboring point within area of $2 \pi \hbar$.

Weirdly enough, the first attempt to define such a quasi-probability distribution, dropped even the first axiom of Kolmogorov, namely that the distribution should be non-negative definite. The Wigner function was this first attempt, introduced by Wigner in 1932 [76], which can give negative probability values. This property though is not unphysical; it is connected with the fact that probability waves can interfere in quantum mechanics. Regardless, the phase space formulation of quantum mechanics is to this day a widely used and powerful semiclassical technique. For excellent reviews, we refer the reader to [73, 79, 137].

\subsubsection{The Husimi $Q$ Distribution Function}

The Husimi quasi-probability distribution function is a well known [137] phase space representation of quantum mechanics. It can be directly connected with the Wigner function, in ways that we show in sec. 2.6.2, and was first introduced by Kodi Husimi in 1940 [77]. To avoid redundancy, in the following we will refer to this quasi-probability distribution function simply as "Husimi function". The advantage of the Husimi function is that it does not have negative values and thus can be interpreted as a probability distribution. This (I believe) is the reason that it has been used extensively in numerous fields such as quantum optics [79, 80, 78], ocean acoustics [81] and quantum chaos [82, 83, 84, 85, 86, 87, 88, 89, 90, 91]. Adaptation in condensed matter physics is still lagging behind other fields, as only recently the Husimi function has been used to study nanodevices [92, 93, 94]. 
One way to define the Husimi function is as an expectation value of the density operator. Given a wavefunction ket $|\psi\rangle$ the operator is defined as $\hat{\rho}:=|\psi\rangle\langle\psi|$. The reason this operator is labeled as "density", comes from the fact that e.g. the position distribution function is the expectation value of the operator in position representation

$$
\langle x|\hat{\rho}| x\rangle=\left\langle x|\psi|\langle\psi \mid x\rangle=|\langle x \mid \psi\rangle|^{2}=\rho(x) .\right.
$$

The Husimi function is defined as the expectation value of $\hat{\rho}$ in a basis of coherent states $\langle\mathcal{W}|\hat{\rho}| \mathcal{W}\rangle$. Equivalently, it can be considered a projection of a wavefunction to a coherent state $\left|\mathcal{W}\left(\mathbf{r}_{0}, \mathbf{k}_{0}, \sigma\right)\right\rangle$.

Formally, these states are eigenstates of annihilation operators. Here, we will not care about such properties, and simply state that coherent states are Gaussian wavepackets: Gaussian envelopes in space with origin $\mathbf{r}_{0}$, which multiply a plane wave with wavevector $\mathbf{k}_{0}$. The key property of coherent states is that they minimize the uncertainty relation between position and momentum.

In position representation and in the absence of magnetic fields, we define the wavepackets as

$$
\mathcal{W}\left(\mathbf{r}_{0}, \mathbf{k}_{0} ; \sigma\right):=\left\langle\mathbf{r} \mid \mathcal{W}\left(\mathbf{r}_{0}, \mathbf{k}_{0} ; \sigma\right)\right\rangle=N_{\sigma}^{D / 2} \exp \left(-\frac{\left(\mathbf{r}-\mathbf{r}_{0}\right)^{2}}{4 \sigma^{2}}+i \mathbf{k}_{0} \cdot \mathbf{r}\right)
$$

with $N_{\sigma}=(\sigma \sqrt{2 \pi})^{-1}$ being the normalization in factor in the continuous case. Formally, the projection of a wavefunction $\psi$ is then defined as [137]

$$
\left\langle\psi \mid \mathcal{W}\left(\mathbf{r}_{0}, \mathbf{k}_{0} ; \sigma\right)\right\rangle=\int \psi^{*}(\mathbf{r}) \times\left\langle\mathbf{r} \mid \mathcal{W}\left(\mathbf{r}_{0}, \mathbf{k}_{0} ; \sigma\right)\right\rangle d \mathbf{r}
$$

where the integral stands for integration across each of the $D$ spatial dimensions and over all the available space. That is also the reason for having $D / 2$ in the exponent of (2.6.1), so that $\langle\mathcal{W} \mid \mathcal{W}\rangle=1$. For our case $D=2$ and thus $\mathbf{r}=(x, y)$. In (2.6.1) we have also assumed equal uncertainties in both spatial directions, $\Delta x=\Delta y=\sigma$.

For a tight-binding system the projection has to be modified to accommodate the discrete nature of the lattice. This is done by turning the integral into a sum over all the lattice sites

$$
\left\langle\psi \mid \mathcal{W}\left(\mathbf{r}_{0}, \mathbf{k}_{0}, \sigma\right)\right\rangle=\sum_{i} \psi^{*}\left(\mathbf{r}_{i}\right) \times e^{-\frac{\left(\mathbf{r}_{i}-\mathbf{r}_{0}\right)^{2}}{4 \sigma^{2}}} e^{i \mathbf{k}_{0} \cdot \mathbf{r}_{i}}
$$

with $\psi\left(\mathbf{r}_{i}\right) \equiv \psi_{i}$ being the wavefunction at lattice site $i$ with position $\mathbf{r}_{i}$. The normalization factors here depend on the lattice and are thus skipped.

The Husimi distribution function is then defined as [137]

$$
Q[\psi]\left(\mathbf{r}_{0}, \mathbf{k}_{0} ; \sigma\right)=\frac{1}{\pi}\left|\left\langle\psi \mid \mathcal{W}\left(\mathbf{r}_{0}, \mathbf{k}_{0}, \sigma\right)\right\rangle\right|^{2} .
$$

In the field of quantum optics, the Husimi function is referred to as $Q$-function [79], which is why in the following we will use the same symbol to represent it. In the following subsections we state some formal properties of the Husimi function, in order to complement the paper of ch. 6 , which only has a brief introduction.

\subsubsection{Connection with the Wigner function}

The definition of the Wigner function $W$ is [73]

$$
\begin{aligned}
W(x, p) & =\frac{1}{2 \pi \hbar} \int_{-\infty}^{+\infty} \exp \left(-\frac{i}{\hbar} p \lambda\right)\left\langle x+\frac{\lambda}{2}|\hat{\rho}| x-\frac{\lambda}{2}\right\rangle d \lambda \\
& =\frac{1}{2 \pi \hbar} \int_{-\infty}^{+\infty} \exp \left(-\frac{i}{\hbar} 2 p y\right) \psi^{*}(x-y) \psi(x+y) d y
\end{aligned}
$$


(for simplicity we will consider one dimension in this section). $Q$ can be connected with $W$ in many (equivalent) ways, one of which is a Weirstrass transform of $W$. This transform is simply a "smoothing" of a function $f$, obtained by averaging the values of $f$ with a Gaussian kernel,

$$
\mathcal{T}_{x}[f](x)=\frac{1}{2 \sigma \sqrt{\pi}} \int_{-\infty}^{+\infty} f(x-y) e^{-\frac{y^{2}}{4 \sigma^{2}}} d y .
$$

In eq. (2.6.6) we are defining the generalized transform which is parameterized by $\sigma$, the "smoothing amount". Then $Q$ is the double Weirstrass transform of $W$

$$
\begin{aligned}
Q(x, p) & =\mathcal{T}_{x} \mathcal{T}_{p}[W](x, p) \\
& \propto \int_{-\infty}^{+\infty} \int_{-\infty}^{+\infty} \exp \left(-\frac{1}{4 \sigma^{2}}\left(x-x^{\prime}\right)^{2}-\frac{\sigma^{2}}{\hbar^{2}}\left(p-p^{\prime}\right)^{2}\right) W\left(x^{\prime}, p^{\prime}\right) d x^{\prime} d p^{\prime}
\end{aligned}
$$

\subsubsection{Connection with the flux operator}

Mason, Borunda and Heller have also used the Husimi function recently in condensed matter systems [92, 93, 94]. The extended the Husimi function, defining the Husimi processed map, which is a tool that allows one to visualize classical paths in nanodevices. Besides this, they also showed how this extension generalized the probability flux operator and allowed it to be used in cases where traditionally the probability flux is everywhere zero to uncover more information about the system.

\subsubsection{Plane Wave example}

The simplest case of the Husimi function of a plane wave is easy to calculate analytically and can provide intuition for the Husimi function. To do this we need to obtain the coherent projection of $P(\mathbf{r}, \mathbf{k})=\exp (i \mathbf{k} \cdot \mathbf{r})$, which equals

$$
\left\langle P \mid \mathcal{W}\left(\mathbf{r}_{0}, \mathbf{k}_{0}, \sigma\right)\right\rangle=4 \pi \sigma^{2} e^{-k_{x}^{\prime 2} \sigma^{2}} e^{-k_{y}^{\prime 2} \sigma^{2}} e^{i k_{x}^{\prime} x_{0}} e^{i k_{y}^{\prime} y_{0}}
$$

using the known integral

$$
\int_{-\infty}^{+\infty} e^{-\frac{\left(x-x_{0}\right)^{2}}{4 \sigma^{2}}} e^{i k x} d x=2 \sigma \sqrt{\pi} e^{-k^{2} \sigma^{2}} e^{i k x_{0}}
$$

This then results in the following Husimi function

$$
Q[P]\left(\mathbf{r}_{0}, \mathbf{k}_{0} ; \sigma\right) \propto\left|\left\langle P(\mathbf{r}, \mathbf{k}) \mid \mathcal{W}\left(\mathbf{r}_{0}, \mathbf{k}_{0}, \sigma\right)\right\rangle\right|^{2} \propto e^{-2 k_{x}^{\prime 2} \sigma^{2}} e^{-2 k_{y}^{\prime 2} \sigma^{2}}
$$

with $k^{\prime}=k-k_{0}$. As expected the Husimi distribution does not depend on $\mathbf{r}_{0}$, since a plane wave is spatially extended. In addition, it depends only on the difference between the measurement wavevector and the plane wave direction which is also something intuitively obvious.

\subsubsection{Zig-Zag Lead Mode example}

Lead modes are crucial components of quantum transport and have been summarized in sec. 2.3.7. For the simplest case of a zigzag nanoribbon in the absence of magnetic fields, the wavefunctions in the continuum description can be expressed as $\Phi=\sin \left(k_{n} y+\alpha\right) e^{i k_{x} x}$ for either sublattice (see eq. 2.3.30). Thus we only need to calculate $\int_{0}^{L} \sin \left(k_{n} y+\alpha\right) e^{-\frac{\left(y-y_{0}\right)^{2}}{4 \sigma^{2}}} e^{i k_{0, y} y} d y$ since we know from the previous calculation that the integral in the $x$ direction is simply $2 \sqrt{\pi} \sigma e^{-k_{x}^{\prime 2} \sigma^{2}} e^{i k_{x}^{\prime} x_{0}}$. Considering the fact that $\sin (a)=(\exp (i a)-\exp (-i a)) / 2 i$, the calculation is straight-forward. 
For the case of very large systems where the integration limits can become $(0, \infty)$ (and also setting $\alpha=0$ for simplicity) we obtain

$$
\begin{aligned}
\int_{0}^{\infty} \sin \left(k_{n} y\right) \mathcal{W}\left(\mathbf{r}_{0}, \mathbf{k}_{0}, \sigma\right) d y & =\frac{1}{2} \frac{1}{2} \int_{-\infty}^{\infty}\left(e^{i k_{n} y}-e^{-i k_{n} y}\right) \exp \left(\frac{\left(y-y_{0}\right)^{2}}{4 \sigma}+i k_{0, y} y\right) d y \\
& =\frac{2 \sigma \sqrt{\pi}}{4}\left(e^{-\left(k_{0, y}+k_{n}\right)^{2} \sigma^{2}}-e^{-\left(k_{0, y}-k_{n}\right)^{2} \sigma^{2}}\right) e^{i \beta}
\end{aligned}
$$

where we have grouped in $e^{i \beta}$ all purely imaginary exponentials that will not appear in the Husimi function due to the presence of the absolute value.

The above result is straight-forward to interpret; just like in the case of the plane wave, there is no dependence on $x_{0}, y_{0}$, since we considered an infinite system. For finite size there would be some small dependence on $y_{0}$ : the Husimi function will be smaller when $y_{0}$ is near the lead edges. The most crucial part is that, even though for the translationally invariant direction (here $x$ ) we recover the result of the plane wave, the case is different for the transverse direction (y). Both wavenumbers $k_{n},-k_{n}$ will gave same result in the Husimi distribution. This means that examining the projection at a test wavevector angle $\theta_{0}$ and $-\theta_{0}$ will give identical results. 


\section{3 | $1^{\text {st }}$ paper: Robustness of ballis- tic transport in antidot superlat- tices}

This paper is published in New Journal of Physics with license CC BY 3.0 as: G. Datseris, T. Geisel and R. Fleischmann, Robustness of ballistic transport in antidot superlattices, New Journal of Physics 21043051 (2019), DOI: 10.1088/1367-2630/ab19cc.

Author contributions All authors conceptualized the project. GD wrote the computer code, did the simulations, data analysis, and the collision time proof. RF created the equations of the stochastic model. GD prepared the figures and the first version of the manuscript. All authors discussed the results as well as reviewed and edited the manuscript. 


\section{New Journal of Physics}

The open access journal at the forefront of physics
OP Institute of Physics
Published in partnership with: Deutsche Physikalische

Gesellschaft and the Institute of Physics

PAPER

CrossMark

\section{Robustness of ballistic transport in antidot superlattices}

OPEN ACCESS

RECEIVED

1 February 2019

REVISED

31 March 2019

ACCEPTED FOR PUBLICATION

16 April 2019

PUBLISHED

30 April 2019

Original content from thi work may be used under the terms of the Creative

Commons Attribution 3.0 licence.

Any further distribution of this work must maintain

attribution to the author(s) and the title of the work, journal citation and DOI.

\author{
George Datseris (iD , Theo Geisel and Ragnar Fleischmann (iD \\ Max Planck Institute for Dynamics and Self-Organization, Germany \\ E-mail: george.datseris@ds.mpg.de
}

Keywords: antidots, Kac's lemma, nonlinear resonance, graphene, measure theory, energy conservation

\begin{abstract}
The magneto-resistance of antidot lattices shows pronounced peaks, which became a hallmark of ballistic electron transport. While most studies agree that they reflect the interplay of regular and chaotic motion in the quasi-classical dynamics, the exact mechanism has been surprisingly controversial. Inspired by recent experiments on graphene antidot lattices showing that the effect survives strong impurity scattering, we give a new explanation of the peaks linked to a fundamental relation between collision times and accessible phase space volumes, accounting for their robustness. Due to the fundamental nature of the mechanism described it will be relevant in many mesoscopic transport phenomena.
\end{abstract}

Antidot superlattices are nanostructured artificial crystals of repellers in high mobility two-dimensional electron gases (2DEGs). They were first fabricated by ion-beam implantation [1] or by etching periodic arrays of holes with periods of a few hundred nanometers into the 2DEG of AlGaAs/GaAs heterostructures [2] and by modulation of the 2DEG by nanostructured lateral metal gates [3]. Since then antidots were realized in many different materials [4-6], and recently also in graphene [7-9] and topological insulators [10]. They are a prime example of devices showing features of ballistic transport: when the typical length scales of a device become smaller than the mean free path of the electrons, transport is no longer dominated by diffusion due to impurity, phonon or electron-electron scattering. Instead, transport is mainly affected by external forces or the potential of the device superstructure.

The most prominent of a number of ballistic transport effects observed in antidot lattices is their low temperature magneto-resistance at small magnetic fields which typically shows a series of pronounced peaks. It was realized [2] that they occur at magnetic field values where collision-less circular cyclotron orbits encircling a certain numbers of antidots can exist in the superlattice (see figures 1 and 2) and they are therefore known as commensurability peaks (CPs). At even lower temperatures, when the coherence length of the electrons grows, quantization of periodic orbits manifests itself in additional resistivity oscillations [11-13]. CPs in graphene have also been reproduced recently in tight-binding simulations of small antidot systems [14]. In the following we will consider the incoherent ballistic transport, which can be analyzed in terms of quasi-classical dynamics [15]. Such quasi-classical resistivity peaks have even been observed at high magnetic fields in the fractional quantum hall regime, where compound quasi-particles, so called composite fermions, experience a reduced effective magnetic field $[16,17]$.

While most studies agree that the CPs reflect the interplay of regular and chaotic quasi-classical dynamics, their origin has remained controversial [15, 18-21]. Recent experiments by Sandner et al [7] on antidot lattices in monolayer graphene can give us new insight, as they reveal the existence of CPs despite strong impurity scattering. In the present letter we analyze the quasi-classical electron dynamics in graphene antidot lattices to interpret these magnetoresistance experiments and provide a new explanation for the mechanisms giving rise to CPs in general.

The first mechanism proposed to explain CPs was based on the assumption that cyclotron orbits encircling antidots get pinned by the antidots and do not contribute to conduction [2]. It was shown theoretically that nonlinear resonances in soft wall models can give rise to such a pinning mechanism [15]. Furthermore it was demonstrated that the resistivity contributions of the chaotic (i.e. non pinned) trajectories alone can already 


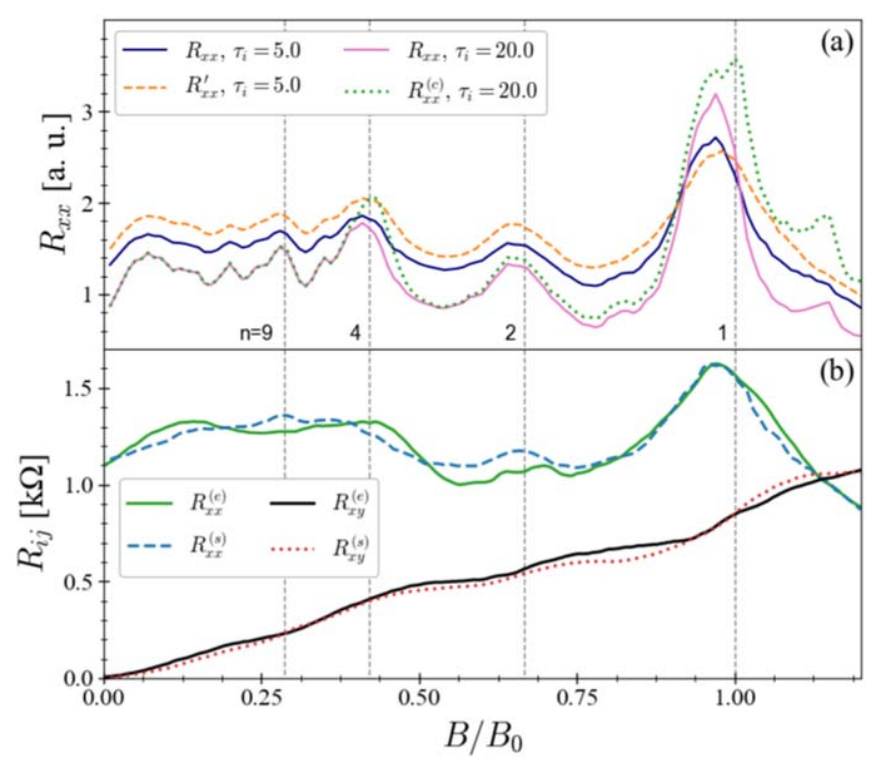

Figure 1. (a): Magnetoresistivity simulations of antidot superlattices (ADSLs) demonstrating commensurability peaks $(c=0.1$, $\left.d_{0}=0.3, \tau_{i}=5,20\right) . R_{x x}^{\prime}$ denotes simulations with boundary roughness with parameter $\varepsilon=0.1$ (see figure 2(b) for mathematical details). $R_{x x}^{(c)}$ is the resistivity of the chaotic part of the phase space. Vertical dashed lines indicate the commensurable fields $B_{n}($ see figure 2). (b) Comparison of recent experiments of magneto- and Hall resistance $R^{(e)}$ by Sandner et al [7] with simulations $R^{(s)}$ using parameters $c=0.2, d_{0}=0.3, \tau_{i}=2.5$ and $B_{0}=3.7 \mathrm{~T}$ (the prefactor was determined by a least squares fit).

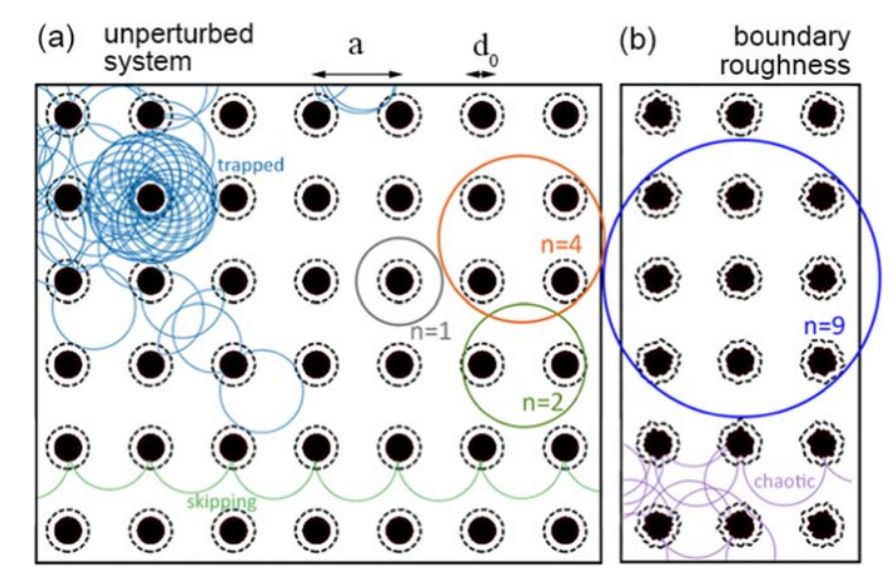

Figure 2. Contours of the potential $U$ (filled: $U \geqslant 1$, dashed: $U=0$ ). Antidots with boundary roughness are shown in (b), with parameter $\varepsilon=0.1$. Examples of chaotic, skipping, as well as trapped orbits at $B=1.0$ and pinned orbits enclosing $n=1,2,4$ and 9 antidots at the commensurable fields $B_{n} \in\{1,0.66,0.42,0.285\}$ are also shown.

produce CPs and that pinning is not the dominant effect [15]. How the chaotic dynamics leads to CPs, however, was strongly disputed (see e.g. [15, 18-21]). In any case one would assume that the mean impurity scattering time $\tau_{i}$ due to residual disorder in the substrate must be sufficiently long in order to observe features of chaotic ballistic dynamics and nonlinear resonances in transport measurements. More specifically it should fulfill $\omega \tau_{i} \gg 2 \pi$, where $\omega$ is the typical frequency of the nonlinear resonances (which in the antidot system is close to the cyclotron frequency $\omega_{c}$ ). In the graphene experiments by Sandner et al [7] and Yagi et al [8], however, the scattering time is quite short and thus $\omega \tau_{i}$ only barely reaches $2 \pi$ (see below for numeric values).

Why do the experiments still exhibit CPs nevertheless? In this article we will show that the resistance peaks survive the impact of these short impurity scattering times due to a deep rooted connection of different dynamical properties. The nonlinear resonances, by their mere existence and simply because they are taking away a part of the chaotic phase space volume, are reducing the fastest chaotic timescale, i.e. the mean time between (successive) collisions with antidots. A fact that we will proof using Kac's lemma. In contrast to the time scales of the nonlinear resonances, the mean collision time in the experiment is shorter or at least comparable to the impurity scattering time, and we argue that its reduction is what is observed in experiment as a resistance peak. The discovered mechanism is very general and will therefore be applicable to a wide range of billiard-like 
mesoscopic systems: generically the intrinsic scattering time of the chaotic orbits is linked to nonlinear resonances simply by their phase space volume.

The paper is structured as follows. In section 1 we first introduce the model we use to describe transport in graphene antidot lattices. Our model uses smooth antidot potentials and includes bulk and boundary disorder. We show that it is capable of quantitatively reproducing the experimental findings very well. In section 2 we analyze the numerical simulations and find that the resistance peaks correspond to minima in the fastest dynamical time scale, the mean collision time. To conclude our analysis and to study this fast timescales in detail, we switch to a billiard model with hard walled antidots. The billiard does not as closely reproduce the experimental findings but allows us to exhibit the underlying dynamical principles much clearer in a well defined and partially analytically treatable manner. We end our discussion by showing in section 2.1 that even in a purely stochastic model of uncorrelated collisions with diffusely scattering antidots the minima in the collision time lead to resistivity peaks. This clearly demonstrates the robustness of the effect.

\section{Model}

We study the electron transport in the single particle picture using (quasi-)classical Hamiltonian dynamics [13]. We therefore model the carriers by a quasi-classical micro-canonical ensemble at the Fermi energy $E_{\mathrm{F}}$. The Hamiltonian is $\mathcal{H}=\mathcal{K}\left(p_{x}, p_{y}\right)+\mathcal{U}(x, y)$, where $\mathcal{K}=v_{\mathrm{F}} \sqrt{p_{x}^{2}+p_{y}^{2}}$ follows from the Dirac approximation of the dynamics of electrons in graphene with Fermi velocity $v_{\mathrm{F}}$ [22]. Our main conclusions apply equally well to the more common quadratic dispersion relation $\mathcal{K} \propto \mathbf{p}^{2}$, as will become apparent later. The magnetic field perpendicular to the graphene layer is introduced by minimal coupling, $\mathbf{p} \rightarrow \mathbf{p}-q \mathbf{A}$, choosing the symmetric gauge $\mathbf{A}=-\frac{1}{2} \mathbf{r} \times \mathbf{B}=\frac{1}{2}(-y B, x B, 0)$. We model the antidot super-lattice by a square array of localized, isotropic potential peaks of the form

$$
U_{a}=\left(\frac{E_{\mathrm{F}}}{c^{4}}\right)\left[\frac{d_{0}}{2}+c-r_{a}\right]^{4}
$$

if $r_{a}=\sqrt{\left(x-x_{a}\right)^{2}+\left(y-y_{a}\right)^{2}}<\frac{d_{0}}{2}+c$ and 0 otherwise. $\left(x_{a}, y_{a}\right)=(n a, m a)$ with $n, m \in \mathbb{N}$ is the center of the (nearest) antidot and $a$ is the super-lattice constant. $d_{0}$ is the antidot diameter (in the experiment by Sandner et al [7] reported values are $d_{0} \approx 25-30 \mathrm{~nm}$ with antidot spacing $a \approx 100 \mathrm{~nm}$ ). The parameter $c$ defines a cut-off distance of the potential and it is also the parameter that controls the steepness of the antidots. The full potential is then the (infinite) sum of the above localized potentials over all antidot centers $\mathcal{U}=\sum_{n, m} U_{a}$. A contour plot of $\mathcal{U}$ is given in figure 2(a). We want to stress that even though of course the diameter and smoothness of the antidots are important parameters, different functional forms (e.g. $\mathcal{U} \propto[\cos (2 \pi x / a) \cos (2 \pi y / a)]^{\beta}$, like in [15]) for the potential have very little impact on the resistivities, and no bearing at all on our main conclusions.

We can add boundary roughness (which aims to model the antidot fabrication defects) to the above potential by making the antidot diameter depend pseudo-randomly on the position. Specifically, let $\tilde{x}=x-x_{a}$, $\tilde{y}=y-y_{a}$ and define $\phi=\arctan (\tilde{y} / \tilde{x})$. We can then write

$$
d(\phi)=d_{0}+d_{0} \varepsilon \sum_{\zeta=1}^{M} \nu\left(x_{a}, y_{a}, \zeta\right) \times \sin (\zeta \phi)
$$

with $\nu$ being random numbers uniformly distributed in $[-0.5,0.5)$ which are different for each combination of $x_{a}, y_{a}, \zeta$, so that on average $\langle d(\phi)\rangle=d_{0}$. M is the total number of sine modes used (measure of the edge complexity) and $\varepsilon$ measures the relative boundary roughness ${ }^{1}$. A realization is shown in figure 2 (b). (Note that this boundary roughness is distinct from the impurity scattering, which we also included in our model, see equation (8).)

Introducing dimensionless variables $x_{i} \rightarrow x_{i} / a$ (where $a$ is the antidot lattice spacing), $v_{i} \rightarrow v_{i} / v_{\mathrm{F}}$ and scaling the energy by the Fermi energy $E_{\mathrm{F}}=\hbar v_{\mathrm{F}} \sqrt{\pi n_{e}}[22](\approx 0.1-1 \mathrm{eV}$ in [7]), the Hamiltonian of our model becomes

$$
\mathcal{H}=\sqrt{\left(p_{x}+y B\right)^{2}+\left(p_{y}-x B\right)^{2}}+\mathcal{U}(x, y) .
$$

In the following we study the dynamics on the manifold $H=1$ (i.e. the Fermi energy). We scale the magnetic field by its value at the principal $(n=1)$ commensurability $B_{0}=2 \hbar \sqrt{\pi n_{e}} /(e a)$, corresponding to a cyclotron diameter equal to the antidot lattice constant $a$. All times are given in units of $t_{0}=a / v_{\mathrm{F}}$ and the cyclotron frequency is $\omega_{c}=2 B$.

Equation (3) leads to the equations of motion $\dot{\mathbf{x}}=\partial \mathcal{H} / \partial \mathbf{p}, \dot{\mathbf{p}}=-\partial \mathcal{H} / \partial \mathbf{x}$. We then obtain equations for velocities instead of momenta

\footnotetext{
${ }^{1}$ In the numerical computations we used an array of $100 \times M$ random numbers repeated periodically and used $M=16$ for all simulations shown in the manuscript.
} 


$$
\begin{gathered}
\dot{x}=v_{x}, \\
\dot{y}=v_{y}, \\
\dot{v}_{x}=v_{y}\left(\frac{v_{x} \frac{\partial \mathcal{U}}{\partial y}-v_{y} \frac{\partial \mathcal{U}}{\partial x}+2 B}{1-\mathcal{U}}\right), \\
\dot{v}_{y}=-v_{x}\left(\frac{v_{x} \frac{\partial \mathcal{U}}{\partial y}-v_{y} \frac{\partial \mathcal{U}}{\partial x}+2 B}{1-\mathcal{U}}\right) .
\end{gathered}
$$

Equations (4)-(7) describe (classical) hyper-relativistic particles in magnetic field $B$ and potential $\mathcal{U}$. The velocity timeseries are obtained by numerical integration of the equations of motion using standard Runge-Kutta schemes.

After obtaining the velocity time series we then calculate the conductivities and resistivities using the Kubo formalism [23]. Following [15] we write

$$
\begin{gathered}
\sigma_{i j} \sim \int_{0}^{\infty} \mathrm{e}^{-t / \tau_{i}}\left\langle v_{i}(t) v_{j}(0)\right\rangle_{E_{\mathrm{F}}} \mathrm{d} t, \\
R_{i j}=\frac{\sigma_{i j}}{\sigma_{x x}^{2}+\sigma_{x y}^{2}}
\end{gathered}
$$

with the conductivities $\sigma_{i j}\left(i j=x x\right.$ or $x y$ ) and the magneto- and Hall-resistivity $R_{x x}$ and $R_{x y}$, respectively (modulo some geometry prefactor). $C_{i j}(t) \equiv\left\langle v_{i}(t) v_{j}(0)\right\rangle_{E_{\mathrm{F}}}$ is the velocity correlation function (VCF), averaged over the available phase space at the Fermi energy. Impurity scattering is introduced in equation (8) by assuming that the electron velocities are decorrelated by random scattering events which follow a Poisson distribution with mean time $\tau_{i}$ [15]. In the experimental paper [7], the authors, using a simple model, estimated $\tau_{i} \approx 3.5$, however the best fit to our more elaborated numerical model yields $2.5 \pm 0.25$ (see below). We will therefore assume the latter to be the correct value. As $\omega_{c} \tau_{i}<2 \pi$, we cannot assume that the regular orbits are actually pinned (with $\sigma_{i j}^{(r)}=0$ as in [15]), since they get scattered before they close. We therefore treated them within the Kubo formalism. They lead to Drude like contributions. By assuming $v_{x}=\cos (2 B t)$ and $v_{y}=\sin (2 B t)$ (i.e. regular cyclotron motion) the VCFs for the regular phase space are $C_{x x}^{(r)}(t)=\cos (2 B t) / 2$ and $C_{x y}^{(r)}(t)=\sin (2 B t) / 2$.

\section{Analysis}

In the following discussion it is advantageous to divide the VCFs into contributions of the different regions of the mixed phase space. The chaotic part of the phase space has portion $g_{c}$ and its correlations $C_{i j}^{(c)}$ are decaying. On the other hand, the regular phase space with portion $g_{r}$ (where $g_{c}+g_{r}=1$ ) has non-decaying correlations $C_{i j}^{(r)}$. We thus write $C_{i j}=\left(1-g_{c}\right) C_{i j}^{(r)}+g_{c} C_{i j}^{(c)}$, and subsequently

$$
\sigma_{i j}=\left(1-g_{c}\right) \sigma_{i j}^{(r)}+g_{c} \sigma_{i j}^{(c)} \text {. }
$$

Note that the regular islands of pinned orbits (see figure 2) correspond to almost circular (quasi-)periodic orbits, which in a smooth antidot potential are stable against the application of an external electric field [15]. Examples of these pinned orbits at the first four commensurabilities are shown in figure 2. Islands can also correspond to various forms of skipping [18] orbits, an example is also shown in the figure. It has been argued that skipping orbits are essential for the creation of resistance peaks [18-21]. Boundary roughness, which probably is present in the experiments of [7], however, almost completely eliminates their existence without affecting the pinned orbits much.

Figure 1(a) shows example magnetoresistivity curves for the unperturbed antidot lattice for various values of the impurity scattering time $\tau_{i}$. An excellent fit to the experiment in magneto- and Hall resistivity is achieved for a small scattering time $\tau_{i}=2.5$, as shown in figure 1(b). Notice that the 4-peak is observable in both experiments and simulations, with characteristic ballistic time scale $T_{R} \approx \pi / 0.44 \approx 7.1$ (in our units). This means that this ballistic feature is observable even when the impurity time is almost three times shorter than the associated ballistic time scale.

The magnetoresistivity of an antidot lattice with boundary roughness $\left(R_{x x}^{\prime}\right)$, shown in figure 1 (a) exhibits surprisingly little differences from the unperturbed lattice. This suggests that the skipping orbits have little impact on the CPs. Furthermore, the resistivity curve $R_{x x}^{(c)}$ of only the chaotic part of phase space, already fully reveals all CPs. It thus suffices to understand how the chaotic correlations change with the magnetic field to produce CPs. 

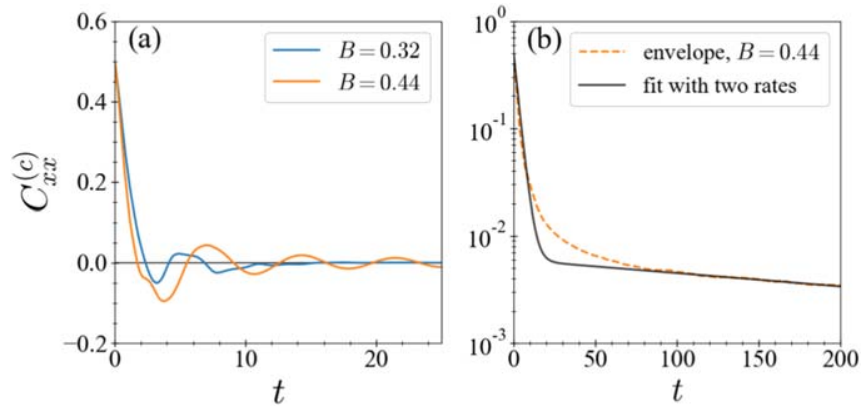

Figure 3. (a): Velocity correlation function of chaotic orbits for $B=0.32,0.44$ and $c=0.1, d_{0}=0.3$. (b): Envelope of $C_{x x}^{(c)}$ and a plot of $p \exp \left(-t / \tau_{\text {fast }}\right)+(1-p) \exp \left(-t / \tau_{\text {slow }}\right)$ with $p=0.985, \tau_{\text {fast }}=3.0, \tau_{\text {slow }}=350.0$. While for non-commensurate magnetic fields $(B=0.32)$ there is a fast exponential decay, for commensurate magnetic fields $(B=0.44)$ there is an additional slow decay as seen in the envelope.

Chaotic orbits get trapped near regular islands [15, 24-28], i.e. they follow the quasiperiodic motion of the regular orbits for long times, as illustrated in figure 2(a). This leads to long-time tails in the correlations of the chaotic orbits. It was therefore argued that they would give rise to valleys rather than peaks in the magnetoresistivity [20]. We will show, however, that this effect is overcompensated by an initially accelerated correlation decay as a direct consequence of phase space volume conservation.

To do so, let us first inspect examples of the chaotic VCFs $C_{i j}^{(c)}(t)$ in figure 3. In the absence of regular islands in phase space $(B=0.32)$ we observe a single, fast correlation decay. In the presence of islands $(B=0.44)$, we see long time tails in the correlations, originating from trapping. From figure 3(b), showing the envelope of the autocorrelation, however, it becomes clear, that the decay is an overlap of a fast and a slow component. We may think of it in the form

$$
C_{x x}^{(c)}(t) \approx g_{\text {col }} C_{\text {fast }}(t)+g_{\text {trap }} C_{\text {slow }}(t)
$$

(and similarly for the cross-correlation $C_{x y}$ ) where $g_{\text {trap }}$ is an appropriate measure of the trapping regions in the chaotic sea, and $g_{\text {col }}=g_{c}-g_{\text {trap }}$ is its complement, i.e. the phase space region with strong chaotic scattering at the antidots ('collisions'). The black line of figure 3(b) illustrates this division by assuming exponential correlation decays with a fast and a slow time constant, $\tau_{\text {fast }}=3$ and $\tau_{\text {slow }}=350$, with $g_{\text {trap }}=1.5 \%$. At this point, the detailed values are of no concern. What we see is that $\tau_{\text {slow }}$ is orders of magnitude larger than $\tau_{i}$. The slow decay rate is therefore almost zero in comparison and the portion of trapped chaotic orbits is just a (small) correction to the portion of pinned orbits. The main contribution to the CPs stems from the change in the fast decay, which reflects the dynamics of the highly chaotic part of the phase space, a finding which is also reported by recent quantum simulations on graphene antidots by Power et al [14]. This is also in accordance with the magnetic focusing mechanism between successive collisions with an antidot and its neighbors that has been argued to increase the diffusivity of chaotic orbits in [29]. The fast correlation decay can be studied best in a billiard model with infinitely steep antidot walls, like in the Sinai billiard [30], which allows for an analytical treatment and should be a good approximation for these highly chaotic trajectories.

In the remainder of this letter we will therefore study the periodic Sinai billiard(PSB), also known as periodic Lorentz gas, which is probably the most prominent example of a low-dimensional ergodic system [2, 30, 31]. The PSB is the infinite steepness limit of the antidot super-lattice: the particles perform true free flight and are reflected specularly when colliding with the disks (i.e. the antidots). We simulate the PSB using an open source software we developed [32]. We denote the collision times in the PSB by $t_{\kappa}$ and the mean collision time by $\kappa$, having in mind that the time scale of the fast correlation decay $\tau_{\text {fast }}$ in the original model is approximated by $\kappa$.

Figure 4(a) shows $\kappa$ as a function of the magnetic field. Remarkably, it exhibits pronounced valleys at the magnetic field values of the CPs in $R_{x x}$ as shown in figure 4(b) for the billiard model. In the whole $B$ range $\kappa$ is comparable with $\tau_{i}$ and even smaller at the commensurable fields. The valleys in $\kappa(B)$ are the origin of the CPs. It is intuitive that more frequent collisions lead to reduced conductivity and thus increased $R_{x x}$, and we will further confirm this argument below using a simplified stochastic model. But first we show that the structure of $\kappa(B)$ has a deep connection with the mixed nature of the phase space (i.e. the coexistence of regular and chaotic phase space regions): figure 4(a) indeed shows that it is directly proportional to the portion of the chaotic part of phase space $g_{c}(B)$.

We can understand this striking fact using Kac's lemma [33-36], which is a direct consequence of phase space volume conservation in Hamiltonian systems. We obtain a map of the flow $\Phi^{t}$ by discretizing in time, $f:=\Phi^{\Delta t_{\varepsilon}}$, with $\Delta t_{\varepsilon} \sim \varepsilon$. Kac's lemma states that the mean number of iterations $\left\langle N_{\mathcal{S}}\right\rangle$ needed to return to a compact subset $\mathcal{S}$ of the phase space $\mathcal{M}$ is given by 


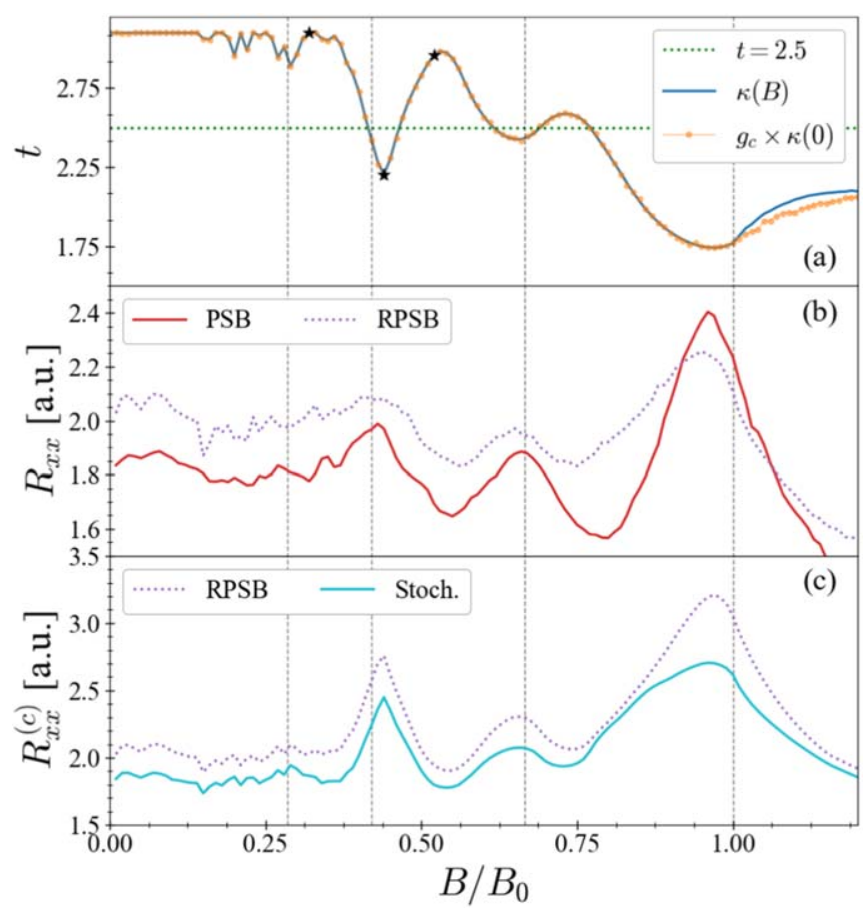

Figure 4. (a): Average collision time $\kappa$ in the periodic Sinai billiard (PSB) versus $B$. It coincides with the portion of chaotic orbits $g$ times $\kappa(0)$. (b) The resistivity curve of the random PSB (RPSB) does not differ much from that of the PSB. (c) The RPSB resistivity curve is approximated sufficiently well by the stochastic model (labeled 'Stoc.'). All curves are calculated with $\tau_{i}=2.5, d_{0}=0.3$.

$$
\left\langle N_{\mathcal{S}}\right\rangle(B)=\frac{\mu\left(\mathcal{M}_{\mathrm{acc}} ; B\right)}{\mu(S ; B)},
$$

where $\mu(\cdot)$ is the phase space measure and $\mathcal{M}_{\text {acc }}$ the part of the phase space accessible to orbits starting in $\mathcal{S}$. Let $\mathcal{T}_{\mathcal{S}}(B)=\Delta t_{\varepsilon} \times\left\langle N_{\mathcal{S}}\right\rangle(B)$ denote physical time instead of map iterations. Let $\mathcal{W}$ be a circle of radius $d_{0} / 2+\varepsilon$ concentric to the antidot and define $\mathcal{S}_{\varepsilon} \subset \mathcal{M}$ such that

$$
\mathcal{S}_{\varepsilon}=\{\vec{x}, \vec{v}: \vec{x} \in \mathcal{W} \text { and } \vec{v} \cdot \vec{\eta}(\vec{x})<0\},
$$

where $\vec{\eta}(\vec{x})$ is the vector normal to $\mathcal{W}$. The mean collision time $\kappa$ of the PBS is exactly $\mathcal{T}_{\mathcal{S}}$ in the limit $\varepsilon \rightarrow 0$.

To find $\mu(\mathcal{S} ; B)$ we first realize that it does not depend on the magnetic field, $\mu(\mathcal{S} ; B)=\mu(\mathcal{S} ; 0)=\mu(\mathcal{S})$. This is due to the infinitesimal width of $\mathcal{S}$, over which motion can always be approximated by a straight line for all finite magnetic fields values (i.e. equaling the magnetic field free case). Then, using (12) at $B=0$, we have

$$
\mathcal{T}_{\mathcal{S}}(0)=\left(\frac{\Delta t_{\varepsilon}}{\mu\left(\mathcal{S}_{\varepsilon}\right)}\right) \mu\left(\mathcal{M}_{\mathrm{acc}} ; 0\right)=\left(\frac{\Delta t_{\varepsilon}}{\mu\left(\mathcal{S}_{\varepsilon}\right)}\right),
$$

because the PSB without magnetic field is fully ergodic and thus $\mu\left(\mathcal{M}_{\text {acc }} ; 0\right)=\mu(\mathcal{M})=1$. By substitution we get $\mathcal{T}_{\mathcal{S}}(B)=\mu\left(\mathcal{M}_{\text {acc }} ; B\right) \times \mathcal{T}_{\mathcal{S}}(0)$. For small enough magnetic fields all chaotic orbits (and up to measure 0 only those) collide with the antidots. In the limit $\varepsilon \rightarrow 0$ both $\Delta t_{\varepsilon}$ and $\mu\left(\mathcal{S}_{\varepsilon}\right)$ go to 0 linearly with $\varepsilon$, therefore their ratio converges, i.e. $\mathcal{T}_{\mathcal{S}} \rightarrow \kappa$. Since by definition $\mu\left(\mathcal{M}_{\text {acc }}\right)=g_{c}$, we find that the mean collision time is given by the fraction of chaotic orbits as a function of the magnetic field $B$, times the mean collision time at $B=0$

$$
\kappa(B)=g_{c}(B) \times \kappa(0) .
$$

From this derivation it becomes also clear that the collision times are not sensitive to boundary roughness. This is intuitive, since the dynamics is already highly chaotic. To confirm this we also simulated a 'rough' Sinai billiard where the particle gets reflected in a random angle at collision with the antidot (RPSB), corresponding to strong boundary roughness. The changes in the magnetoresistivity are only small indeed as shown in figure 4(b).

\subsection{Stochastic model}

For completeness we now conclude our argumentation that the CPs arise due to the collision times by linking the collision times to the resistivities in a simplified purely stochastic analytic model for the motion in the billiard with boundary roughness (RPSB). We approximate the dynamics by a renewal process of stochastic scattering events (the distribution of the scattering times reflects the geometry of the antidot lattice) and free cyclotron motion in between scattering events. Each scattering event with a rough boundary leads to a random change in 
the velocity angle

$$
\alpha=\pi+2 \psi,
$$

where $\psi$ is the random angle formed by the normal of the boundary segment and the velocity vector of the trajectory undergoing the scattering event. Under the assumption that all spatial orientations of the boundary segments are equally probable the probability density of hitting a segment with angle $\psi$ is

$$
h(\psi)=\frac{1}{2} \cos \psi, \text { with } \psi \in[-\pi / 2, \pi / 2] .
$$

Notice that this stochastic model is not the same as the RPSB; in the latter there is a strong correlation between the reflected angle and the subsequent collision time. This correlation does not exist in the stochastic model, as well as any knowledge of phase space volumes.

The VCFs are

$$
\begin{aligned}
& S_{x x}=\left\langle v_{y}(t) v_{x}(0)\right\rangle=v_{0}^{2}\langle\cos (\varphi(t)) \cos (\varphi(0))\rangle, \\
& S_{y x}=\left\langle v_{y}(t) v_{x}(0)\right\rangle=v_{0}^{2}\langle\sin (\varphi(t)) \cos (\varphi(0))\rangle,
\end{aligned}
$$

where $v_{0}$ is the constant absolute value of the velocity $\left(v_{0}=1 \mathrm{in}\right.$ our units) and the ensemble average $\langle\cdot\rangle$ reduces to an average over the initial angle $\varphi_{0}$ of the velocities and the collision events. In the free propagation in-between events the velocity angle changes by $\Delta \varphi(t)=\omega \Delta t$ with $\omega=2 B$ the cyclotron frequency. The contribution to the correlation functions of all trajectories that have scattered (exactly) $m$ times up to time $t$ is given by

$$
\begin{aligned}
& S_{x x}(t \mid m)=P_{m}(t ; B) \int_{-\pi}^{\pi} \mathrm{d} \varphi_{0} \int_{-\pi / 2}^{\pi / 2} \mathrm{~d} \psi_{1} \ldots \int_{-\pi / 2}^{\pi / 2} \mathrm{~d} \psi_{m} \frac{\cos \varphi_{0}}{2 \pi} \cos \left(\omega t+\varphi_{0}+m \pi+2 \sum_{i=1}^{m} \psi_{i}\right) \frac{\prod_{i=1}^{m} \cos \psi_{i}}{2^{m}} \\
& S_{y x}(t \mid m)=P_{m}(t ; B) \int_{-\pi}^{\pi} \mathrm{d} \varphi_{0} \int_{-\pi / 2}^{\pi / 2} \mathrm{~d} \psi_{1} \ldots \int_{-\pi / 2}^{\pi / 2} \mathrm{~d} \psi_{m} \frac{\cos \varphi_{0}}{2 \pi} \sin \left(\omega t+\varphi_{0}+m \pi+2 \sum_{i=1}^{m} \psi_{i}\right) \frac{\prod_{i=1}^{m} \cos \psi_{i}}{2^{m}}
\end{aligned}
$$

where $P_{m}(t ; B)$ is the probability that a trajectory has scattered exactly $m$ times up to time $t$. The $\psi_{i}$ integrals can be easily carried out successively. Using the notation $\phi_{m}=\omega t+\varphi_{0}+m \pi+2 \sum_{i=1}^{m} \psi_{i}$, we write

$$
\begin{aligned}
\cos \left(\phi_{m}\right) & =\cos \left(\phi_{m-1}+2 \psi_{m}+\pi\right) \\
& =-\cos \left(\phi_{m-1}\right) \cos \left(2 \psi_{m}\right)+\sin \left(\phi_{m-1}\right) \sin \left(2 \psi_{m}\right)
\end{aligned}
$$

and similarly

$$
\sin \left(\phi_{m}\right)=-\cos \left(\phi_{m-1}\right) \sin \left(2 \psi_{m}\right)-\sin \left(\phi_{m-1}\right) \cos \left(2 \psi_{m}\right)
$$

With

$$
\begin{aligned}
& \frac{1}{2} \int_{-\pi / 2}^{\pi / 2} \cos (x) \cos (2 x) \mathrm{d} x=\frac{1}{3} \\
& \frac{1}{2} \int_{-\pi / 2}^{\pi / 2} \cos (x) \sin (2 x) \mathrm{d} x=0
\end{aligned}
$$

and

$$
\frac{1}{2 \pi} \int_{-\pi}^{\pi} \cos \left(\varphi_{0}\right) \cos \left(\omega t+\varphi_{0}\right) \mathrm{d} \varphi_{0}=\frac{1}{2} \cos (\omega t)
$$

we find $C_{x x}(t \mid m)=\frac{1}{2}\left(-\frac{1}{3}\right)^{m} \cos (\omega t)$ and $C_{y x}(t \mid m)=\frac{1}{2}\left(-\frac{1}{3}\right)^{m} \sin (\omega t)$. This finally allows us to write the correlation functions as the sum of these contributions:

$$
\begin{aligned}
& S_{x x}(t)=\frac{1}{2} \sum_{m=0}^{\infty}\left(-\frac{1}{3}\right)^{m} P_{m}(t ; B) \cos (\omega t), \\
& S_{y x}(t)=\frac{1}{2} \sum_{m=0}^{\infty}\left(-\frac{1}{3}\right)^{m} P_{m}(t ; B) \sin (\omega t) .
\end{aligned}
$$

The probability density function (pdf) of the collision time $p(t)$ is a complicated function that is very sensitive to changes in the magnetic field, as shown in figure 5. Therefore it is hard to find a useful analytical model for the probability $P_{m}(t ; B)$. Only in the limit of large $m$ they are well approximated by Gaussians (see e.g. [37]). We are, however, mainly interested in short times and thus small $m$. Therefore we choose to calculate the $P_{m}(t ; B)$ numerically using the following method: let $q_{m}(t)$ denote the pdf for the $m$ th scattering event $(m \geqslant 1)$ occuring at time $t$. It can be calculated through recursive convolution with the pdf $p(t)$ 


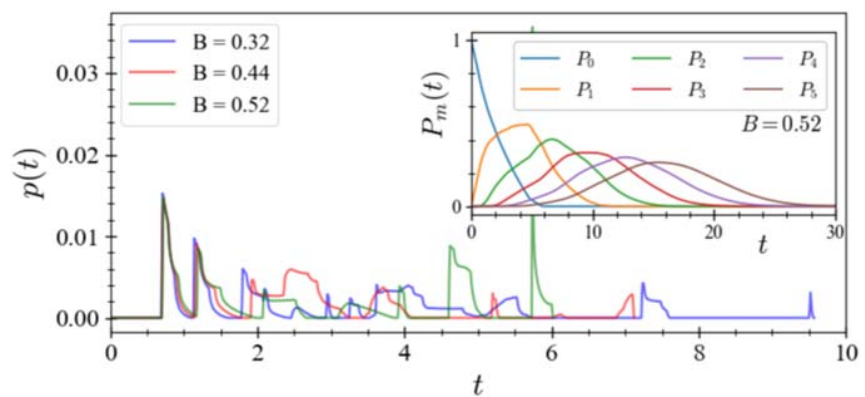

Figure 5. Probability density function of the collision times $p\left(t_{\kappa}\right)$ in the rough periodic Sinai billiard for the magnetic fields values marked by black stars in figure 4 of the manuscript, i.e. $B \in\{0.32,0.44,0.52\}$. The inset shows numerically computed probabilities $P_{m}(t ; B)$ for $B=0.52$.

$$
q_{m}(t)=\int_{0}^{t} \mathrm{~d} z p(z) q_{m-1}(t-z)
$$

with $q_{1}(t) \equiv p(t)$. Then $P_{m}(t)$ can be found from $q_{m}$ using the survival function $W(t)=\int_{t}^{\infty} p\left(t^{\prime}\right) \mathrm{d} t^{\prime}$

$$
P_{m}(t)=\int_{0}^{t} \mathrm{~d} z W(z) q_{m}(t-z)
$$

for $m>0$ and $P_{0}(t) \equiv W(t)$. Examples of $P_{m}(t)$ are shown in the inset of figure 5 .

Our stochastic model reproduces the contribution of the chaotic orbits to the CPs in the RPBS in very good approximation, as shown in figure 4(c). This confirms our claim that the CPs are due merely to the distribution of collision times in the antidot lattice.

\section{Conclusions}

In conclusion, we have explained recent magnetotransport experiments on graphene antidot lattices using appropriate quasiclassical electron dynamics. (And we note that our approach can also be applied to the analysis of the Hall-resistivity which shows a quenched or even negative Hall-effect at very small magnetic fields.) We found that ballistic transport features like the CPs are visible in the resistivities even though the mean free time due to impurity scattering is so short that it is comparable to the fastest timescales of the chaotic dynamics. We showed that this striking robustness of the commensurability features can be understood by the fact that the fast chaotic time scale, the collision time, is reduced by the mere existence of stable islands in a mixed phase space, which reduce the chaotic phase space volume (Kac's Lemma). By this we solved a decades old riddle on the influence of nonlinear resonances on magnetotransport in antidot superlattices. Finally, due to the fundamental nature of the mechanism linking the time scales, which only depends on the basic properties of chaotic Hamiltonian systems with mixed phase space, it will be generally applicable to a wide range of mesoscopic systems.

\section{Acknowledgments}

We thank Andreas Sandner and Jonathan Eroms very much for granting us access to their experimental data and fruitful discussions. We thank Stephan Eule for helpful discussions.

\section{ORCID iDs}

George Datseris (D) https://orcid.org/0000-0002-6427-2385

Ragnar Fleischmann (iD https://orcid.org/0000-0001-6843-8146

\section{References}

[1] Ensslin K and Petroff P M 1990 Phys. Rev. B 4112307

[2] Weiss D, Roukes M L, Menschig A, Grambow P, von Klitzing K and Weimann G 1991 Phys. Rev. Lett. 662790

[3] Lorke A, Kotthaus J P and Ploog K 1991 Phys. Rev. B 443447

[4] Xiao Z L et al 2002 Appl. Phys. Lett. 812869

[5] Wördenweber R, Dymashevski P and Misko V R 2004 Phys. Rev. B 69184504

[6] Neusser S, Botters B and Grundler D 2008 Phys. Rev. B 78054406 
[7] Sandner A, Preis T, Schell C, Giudici P, Watanabe K, Taniguchi T, Weiss D and Eroms J 2015 Nano Lett. 158402

[8] Yagi R, Sakakibara R, Ebisuoka R, Onishi J, Watanabe K, Taniguchi T and Iye Y 2015 Phys. Rev. B 92195406

[9] Oka T, Tajima S, Ebisuoka R, Hirahara T, Watanabe K, Taniguchi T and Yagi R 2019 Phys. Rev. B 99035440

[10] Maier H, Ziegler J, Fischer R, Kozlov D, Kvon Z D, Mikhailov N, Dvoretsky S A and Weiss D 2017 Nat. Commun. 81

[11] Weiss D, Richter K, Menschig A, Bergmann R, Schweizer H, von Klitzing K and Weimann G 1993 Phys. Rev. Lett. 704118

[12] Silberbauer H and Rossler U 1994 Phys. Rev. B 5011911

[13] Richter K 1995 Europhys. Lett. 297

[14] Power S R, Thomsen M R, Jauho A P and Pedersen T G 2017 Phys. Rev. B 96075425

[15] Fleischmann R, Geisel T and Ketzmerick R 1992 Phys. Rev. Lett. 681367

[16] Kang W, Stormer H L, Pfeiffer L N, Baldwin K W and West K W 1995 Physica B 211396

[17] Fleischmann R, Geisel T, Holzknecht C and Ketzmerick R 1996 Europhys. Lett. 36167

[18] Baskin É, Gusev G, Kvon Z, Pogosov A and Entin M 1992 JETP Lett. 55678

[19] Schuster R, Ernst G, Ensslin K, Entin M, Holland M, Böhm G and Klein W 1994 Phys. Rev. B 508090

[20] Fließer M, Schmidt G J O and Spohn H 1996 Phys. Rev. E 535690

[21] Ishizaka S and Ando T 1997 Phys. Rev. B 5516331

[22] Castro Neto A H, Guinea F, Peres N M R, Novoselov K S and Geim A K 2009 Rev. Mod. Phys. 81109

[23] Kubo R 1957 J. Phys. Soc. Japan 12570

[24] Meiss J D and Ott E 1985 Phys. Rev. Lett. 552741

[25] Geisel T, Zacherl A and Radons G 1987 Phys. Rev. Lett. 592503

[26] Geisel T, Zacherl A and Radons G 1988 Z. Phys. B 71117

[27] Tabor M 1989 Chaos and Integrability in Nonlinear Dynamics (New York: Wiley) p 384

[28] Arnold V I 1989 Mathematical Methods of Classical Mechanics, Graduate Texts in Mathematics vol 60 (New York: Springer) (https://doi. org/10.1007/978-1-4757-2063-1)

[29] Ando T, Uryu S and Ishizaka S 1999 Jpn. J. Appl. Phys. 38308

[30] Sinai Y G 1970 Russ. Math. Surv. 25137

[31] Fliesser M, Schmidt G J O and Spohn H 1996 Phys. Rev. E 535690

[32] Datseris G 2017 J. Open Source Softw. 2458

[33] Kac M 1947 Bull. Am. Math. Soc. 20376

[34] MacKay R S 1994 J. Nonlinear Sci. 4329

[35] Meiss J D 1997 Chaos 7139

[36] Altmann E G and Tel T 2008 Phys. Rev. Lett. 100174101

[37] Mitov K V and Omey E 2014 Renewal Processes (Cham: Springer International Publishing) (https://doi.org/10.1007/978-3-03480191-1) 


\subsection{Hall resistance}

In sec. 2.1 we mentioned the quantum Hall effect, where the Hall resistance $\left(R_{H}\right)$ increases in quantized steps. For weak magnetic fields (below the QHE regime), the $R_{H}$ of an ADSL device behaves similarly: it increases in non-quantized steps whenever the magnetoresistance displays peaks (which are linked to the existence of stable orbits) [104, 138]. In this section I want to focus on the behavior of $R_{H}$ for very small magnetic fields (in the units discussed in the first paper somewhere between 0 and 0.1). This is relevant because, as will be shown below, looking at $R_{H}$ gave me the necessary insight to discover the importance of the mean collision time in the first paper.

\subsubsection{Negative Hall effect}

For small $B$ it is known (both via experiments [104] as well as simulations [138]) that $R_{H}$ becomes negative or stays very small (compared with the predicted Drude slope), depending on the mean free time. This is shown in Fig. 3.1. This effect came to be known as negative or "quenched" Hall effect and was examined in detail in [138]. The effect stems from the velocity cross-correlation $C_{x y}$, seen for example in Fig. 3.2, whose integral uniquely determines the sign of $R_{H}$. $C_{x y}$ becomes negative after some initial decay and it was shown in [138] that the negative part of $C_{x y}$ goes to 0 as a power-law, which gives a strong negative contribution to the integral.

This power-law was connected with long channeling orbits, which perform Lévy flights. Typical electron motion for the continuous system and small magnetic fields is shown in Fig. 3.2(c). There we can see that the electron trajectories are very chaotic, but are frequently interrupted by very long, almost completely straight segments, which are called "channels". The length of these channels follows a power-law distribution, a defining characteristic of Lévy flights, but I will not show these results in this thesis for the sake of conciseness. For more details on the subject please see [138].

\subsubsection{Collisions and negative cross-correlation}

Even though in [138] it became clear why the cross correlation has a negative power law decay, what I wanted to understand is why it becomes negative in the first place. The new experiments did not show any sufficient quenching of $R_{H}$, due to the very strong impurity scattering, and yet

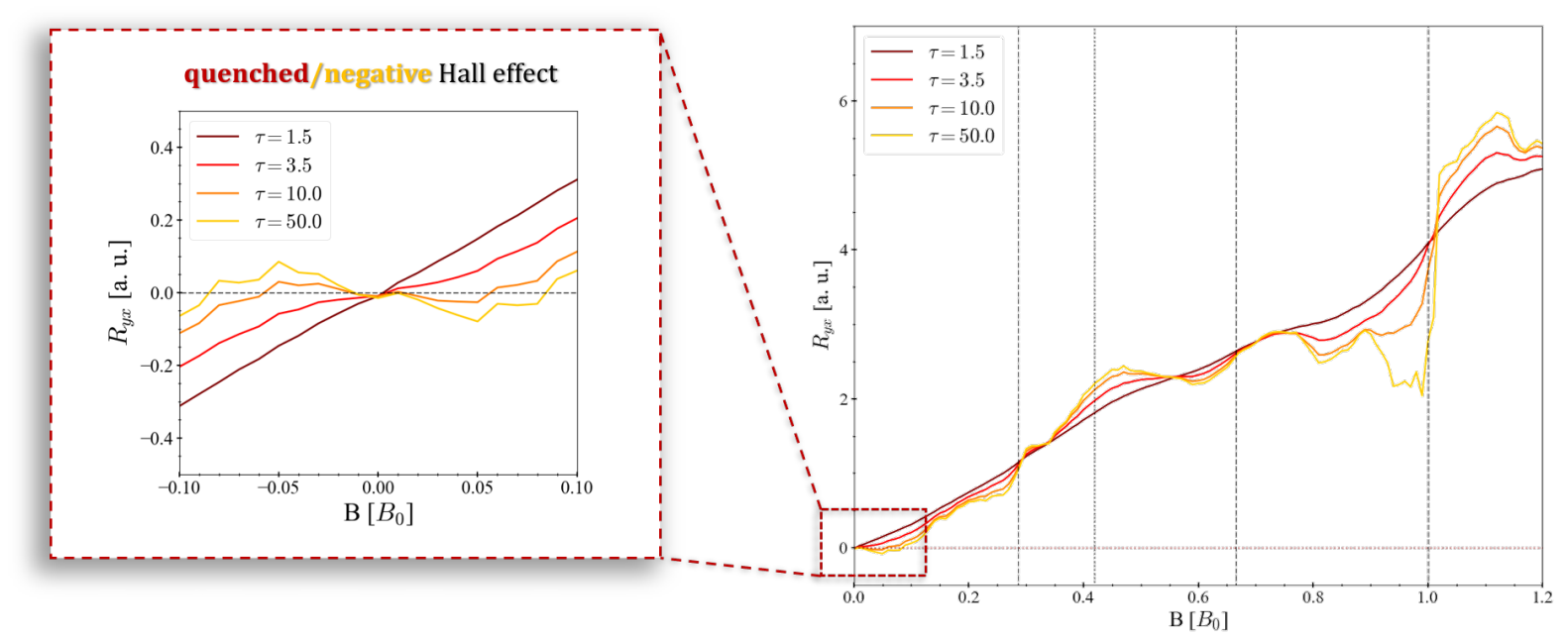

Figure 3.1: Hall resistance $R_{H} \equiv R_{y x}$ of the continuous ADSL system of the first paper, for parameters $c=0.1$ (antidot steepness) and $d=0.3$ (antidot radius) and various impurity scattering times. The zoom in displays the quenched or negative Hall effect, which depends on the strength of the impurity scattering. 
our simulations showed that $C_{x y}$ becomes strongly negative (but the power-law tail is suppressed, like in Fig. 3.2(b)).

For the value of $R_{H}$, the cross-correlation $C_{y x}$ has much more impact than the autocorrelation $C_{x x}$, which decays almost exponentially in this magnetic field regime. What one has to understand is whether there is something special about the exact point in time that $C_{y x}$ becomes 0 . As the magnetic field here is very weak, this point cannot have any connection with oscillations in $C_{y x}$ due to the magnetic field, following the form $\sim \sin (2 B t)$, which exist at larger $B$ values.

When one thinks about the dynamics of the electron velocities in the antidot lattice, one thing can become evident. Colliding with an antidot will, on average, reverse the sign of the product $v_{x} v_{y}$ (the particle's velocities). To understand this let us re-write the velocity correlations in the collision coordinate system. Since the correlation functions are a result averaged over time, and due to the fact that an ADSL possesses the symmetries of the square, it holds

$$
\begin{aligned}
& C_{x x}=\left\langle v_{x}(t) v_{x}(0)\right\rangle=\frac{1}{2}\langle\mathbf{v}(t) \cdot \mathbf{v}(0)\rangle \\
& C_{x y}=\left\langle v_{x}(t) v_{y}(0)\right\rangle=\frac{1}{2}\left\langle[\mathbf{v}(t) \times \mathbf{v}(0)]_{z}\right\rangle
\end{aligned}
$$

(notice that $v_{z}=0$ ). In a collision event the longitudinal component of the velocity changes sign, while the transverse does not. Thus the longitudinal-transverse product $\left(C_{x y}\right)$ changes sign, but the longitudinal-longitudinal $\left(C_{x x}\right)$ does not.

In addition, it is possible to find strong numeric evidence that $C_{x y}$ should change sign on the collision with antidots. Fig. $3.2(\mathrm{~d})$ plots the time $t_{c}$ that $C_{x y}$ crosses zero for various parameters of the continuous ADSL model. It is clear that this time is relatively constant for small magnetic fields, which intuitively is what one would expect. The time between collisions should be impacted very little by a small magnetic field, as the particle motion is relatively the same. In addition, this time is larger for $c=0.1$ then when $c=0.2$. This also falls in line with the idea, since smaller $c$ means smaller effective radius for the antidot (more details in the first paper preceding this section).

(a)

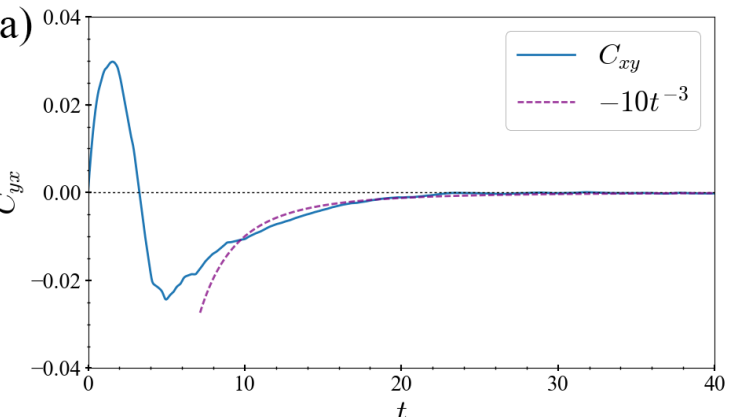

(b)

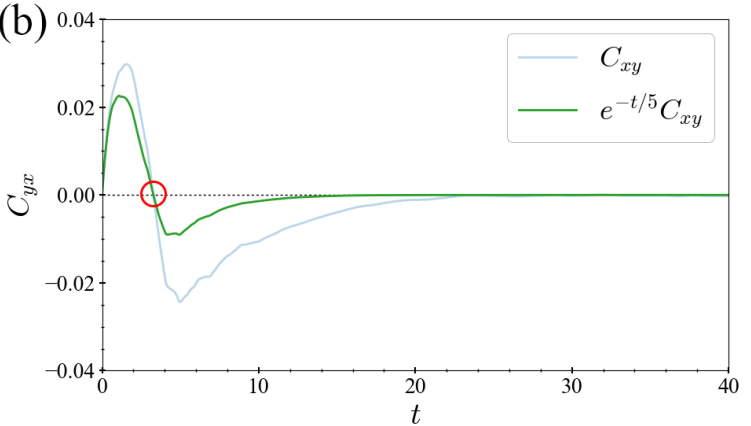

(c)

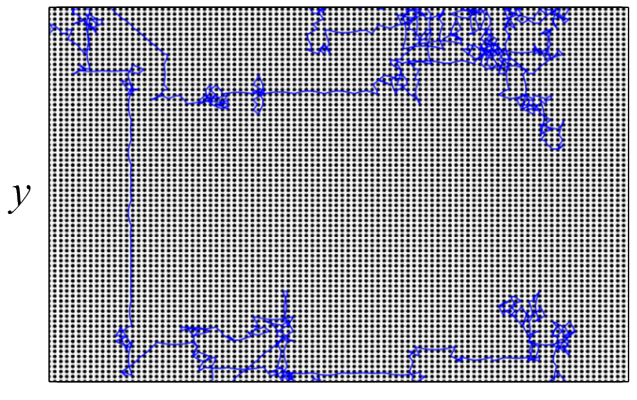

$\mathbf{X}$

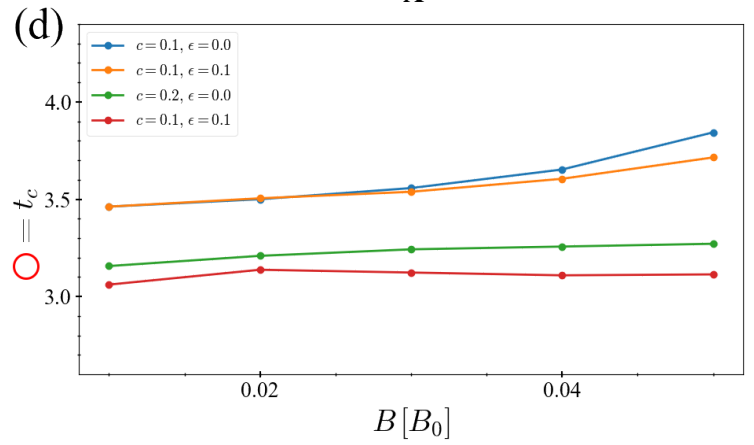

Figure 3.2: ( $a, b)$ Velocity cross-correlation for small magnetic field $B=0.02$. It is shown that a power law fits the negative tail, but this power law disappears for strong impurity scattering. (c) Channeling orbits that lead to the negative power law tail in $C_{x y}$. (d) First zero of the cross-correlation $t_{c}$. 
What was the the decisive evidence however was the numeric value of this time $t_{c}$. We see that it is around 3 to 3.5 depending on the steepness. What is known in the literature is the exact, analytic value for the mean collision time in the periodic Sinai billiard [139]. The expression is $\left(4-\pi d^{2}\right) / 4 d$ which, for $d=0.3$, gives a result of 3.1. This established that it is the collision with the antidots that leads to the cross-correlation becoming negative initially, and later made us appreciate and understand the impact the mean collision time has on the magnetoresistance as well. 


\section{Software to simulate dynamical billiards}

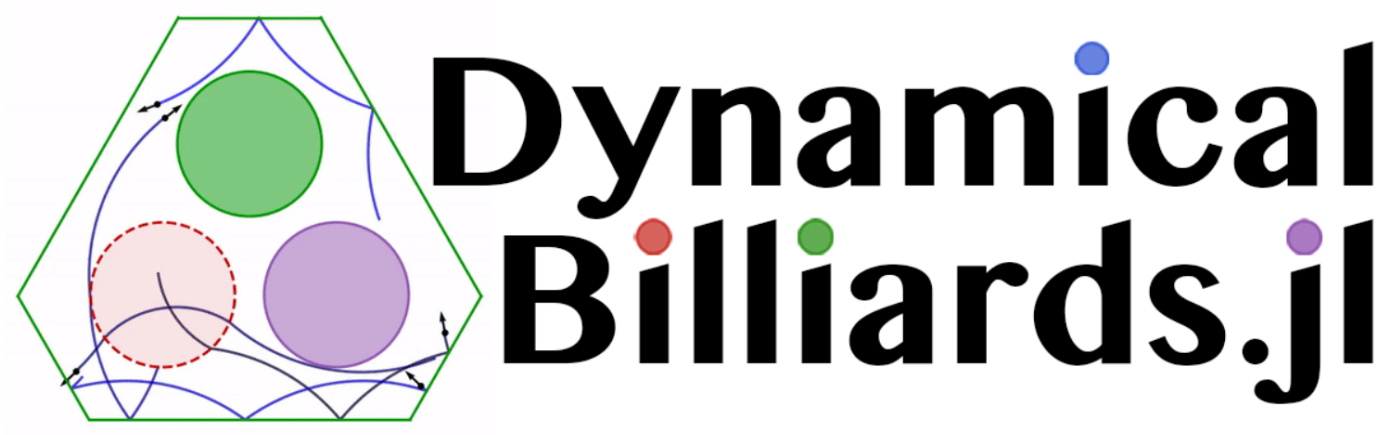

Figure 4.1: Logo of DynamicalBilliards.jl.

While analyzing antidot superlattices (ADSL) I needed to simulate dynamical billiards in two dimensions. To briefly recapitulate, billiards are simple dynamical systems in which a point particle is propagating inside a domain (either closed or periodic), performing "free flight" (movement in the absence of scalar potentials). For the analyses I required a software that could calculate the collision times exactly, included magnetic fields, was extendable (to allow implementing the Lyapunov exponent calculation), was written in a high-level language (for interactivity), and was able to plot billiards. Such software did not exist when I started my project, and so I decided to create it myself. The result is the software DynamicalSystems.jl [123], written in the Julia programming language [140]. In addition, I made it simple to use, free and open source, well documented and with the highest performance possible. Making the software publicly available was an excellent choice, as it allowed it to be used and even be extended by others.

A detailed documentation (circa 100 pages) is available online under this page: https:// juliadynamics.github.io/DynamicalBilliards.jl/dev/. This documentation is guaranteed to be up to date, since it is created from within the software itself, and thus is updated with every change to the source code. Because of the extent of this documentation, this chapter will be very brief. It is worth noting that besides me and my coworkers using this software extensively in two publications, DynamicalBilliards.jl has already been in use by the scientific community, since it has been used, for example, in a recent publication by Tapias, Sanders and Altmann [141] to study statistically extreme trajectories.

\subsection{Design}

DynamicalBilliards.jl is propagating particles in billiards following a completely modular approach. In general, the workflow of DynamicalBilliards.jl follows these simple steps: (1) create a billiard, (2) create particles inside that billiard (3) get the output you want by using 
Notice of course that in the actual simulations of the second manuscript in ch. 5, we did not use random initial conditions. For example for the MPSB we chose initial conditions such that there were no pinned particles (since by definition their Lyapunov exponent is zero). This does not come at the cost of writing much more code though. For example, modifying the above example to only use non-pinned particles as initial conditions can be done as:

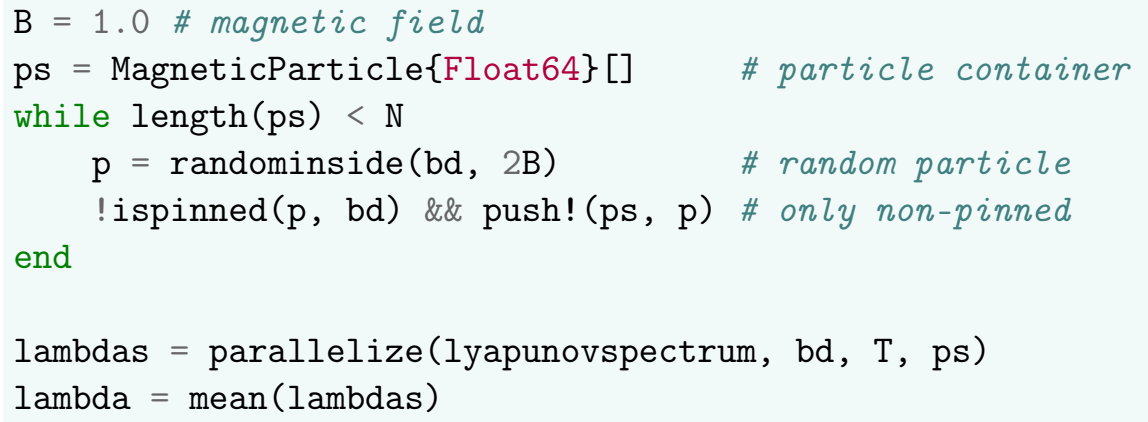

\subsection{7}

\subsection{Unique features}

Another (free) software to simulate billiard systems is Bill2D [142], albeit its scope is a bit different from DynamicalBilliards.jl since it targets interacting electrons. Regardless, out of scanning the available source code and other implementations on the web to simulate billiards, to the best of my knowledge DynamicalBilliards.jl has some unique features that I could not find anywhere. I will list them here but will not discuss them further, since all of them have several pages dedicated to them on the official documentation.

- Extensive documentation available online, with several examples, tutorials, figures, animations \& runnable code snippets, everything hyper-linked with each other.

- Guaranteed up-to-date documentation, because it is generated from within the software and recompiled with any change in the source code.

- Native, fully featured support for plotting and animating the motion of any particle in any billiard.

- An interface to interactively switch from real 3D space (the flow) to boundary 2D space (the map) and also from boundary space back to real space.

- Ray-splitting: particles may propagate an obstacle given arbitrary transmission and refraction laws.

- Lyapunov exponent computation based on the algorithm by Dellago et al. [143].

- Lyapunov exponent computation in the presence of magnetic fields.

- Analytic computation of collision times in magnetic fields.

- Support for creating random initial conditions in an arbitrary billiard. 


\section{$5 \quad 2^{\text {nd }}$ paper: Estimating Lyapunov exponents in billiards}

This paper is published in Chaos with license CC BY 3.0 as G. Datseris, L. Hupe and R. Fleischmann, Estimating Lyapunov exponents in billiards, Chaos 29, 093115 (2019)

DOI: $10.1063 / 1.5099446$.

Author contributions GD and RF conceptualized the project. LH extended the Dellago formalism to magnetic fields. GD proved eq.(1) and LH and GD proved the mean return time to the stem. GD and LH wrote the computer code, did the simulations and data analysis. All authors contributed in creating the final version of the toy model. GD prepared the figures (except figure 8, made by LH) and the first version of the manuscript. All authors discussed the results as well as reviewed and edited the manuscript. 


\title{
Estimating Lyapunov exponents in billiards
}

\author{
Cite as: Chaos 29, 093115 (201 9); doi: 10.1 063/1.5099446 \\ Submitted: 1 OApril 2019 . Accepted: 23 August 2019 . \\ Published Online: 13 September 2019

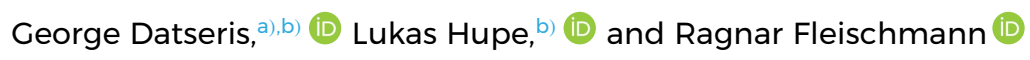 \\ AFFILIATIONS \\ Max Planck Institute for Dynamics and Self-Organization, Am Fassberg 17, 37077 Göttingen, Germany and Faculty of Physics, \\ Georg-August-Universität Göttingen, 37077 Göttingen, Germany \\ a) george.datseris@ds.mpg.de \\ ${ }^{b}$ Contributions: G. Datseris and L. Hupe contributed equally to this work.
}

\begin{abstract}
Dynamical billiards are paradigmatic examples of chaotic Hamiltonian dynamical systems with widespread applications in physics. We study how well their Lyapunov exponent, characterizing the chaotic dynamics, and its dependence on external parameters can be estimated from phase space volume arguments, with emphasis on billiards with mixed regular and chaotic phase spaces. We show that in the very diverse billiards considered here, the leading contribution to the Lyapunov exponent is inversely proportional to the chaotic phase space volume and subsequently discuss the generality of this relationship. We also extend the well established formalism by Dellago, Posch, and Hoover to calculate the Lyapunov exponents of billiards to include external magnetic fields and provide a software on its implementation.

(C) 2019 Author(s). All article content, except where otherwise noted, is licensed under a Creative Commons Attribution (CC BY) license (http://creativecommons.org/licenses/by/4.0/). https://doi.org/10.1063/1.5099446
\end{abstract}

From the foundations of statistical physics to transport properties of electronic devices, in many areas of physics "billiard models" are an important tool for understanding complex dynamics. In a billiard model, a point particle is moving freely (and frictionless) on a flat (or constantly curved) surface until it hits the boundary of the billiard where it is specularly reflected. Chaotic dynamics in the billiard are characterized by a positive "Lyapunov exponent," measuring how initially close trajectories separate exponentially fast. Obtaining its value so far usually requires detailed numerical simulations of the chaotic dynamics. In our paper, we assess how well the Lyapunov exponent can be estimated from quite general considerations. Specifically, we study how parameter changes that vary the phase space structure of the billiard get reflected in the Lyapunov exponent. For example, the application of an external magnetic field can force some trajectories in the billiard on closed cyclotron orbits. We show how the mere existence of such orbits varies the Lyapunov exponent of the chaotic dynamics through the phase space volume they occupy. The knowledge of this connection will be helpful to understand physical mechanisms in many systems like the magnetotransport in graphene nanostructures.

\section{INTRODUCTION}

Dynamical billiards are a well-studied class of dynamical systems, having applications in many different fields of physics. Besides playing a prominent role in ergodic theory, ${ }^{1-3}$ billiards are important example systems for understanding quantum chaos, ${ }^{4,5}$ with practical applications, e.g., in modeling optical microresonators for lasers ${ }^{6,7}$ and room acoustics. ${ }^{8}$ Billiard models have also been particularly successful in helping to understand transport properties of electronic nanostructures such as quantum dots and antidot superlattices. ${ }^{9-18}$

A billiard consists of a finite (or periodic) domain in which a point particle performs free flight with a unit velocity. Upon collision with the boundary of the domain, the particle (typically) is specularly reflected. In Fig. 1, we are showing the two example billiards we will be considering in this paper: the mushroom billiard $(\mathrm{MB})^{19}$ and the periodic Sinai billiard ${ }^{1}$ without (PSB) and with magnetic field (MPSB).

An essential characterization of the chaotic dynamics of a billiard is of course provided by its Lyapunov exponents. (In this article, we will study the Lyapunov exponents of the "billiard flow" in the physical, continuous time, in contrast to those of the "billiard map" in a discrete time that counts the number of collisions with the boundary.) For a two dimensional billiard, the Lyapunov exponents are four numbers $\lambda_{1-4}$ that measure how "chaotic" the billiard is, in terms of the average exponential rates of expansion (and contraction) of the phase space along certain characteristic directions. Due to the Hamiltonian nature of the dynamics, the Lyapunov exponents fulfil $\lambda_{1}=-\lambda_{4}, \lambda_{2}=\lambda_{3}=0$. Therefore, in the remainder of the text, we will be only considering the largest exponent $\lambda \equiv \lambda_{1}$. The fundamental mathematical properties of the Lyapunov exponents 

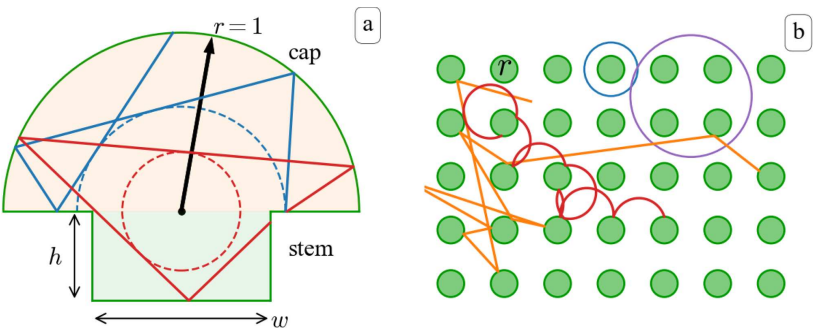

FIG. 1. (a) A regular (blue) and chaotic (red) orbit in the mushroom billiard (MB), whose cap radius is a constant set to 1 and the stem has width $w$ and height $h$. The cap and the stem are separated with different background colors (orange, green). (b) Chaotic orbits without (orange) and with (red) magnetic field and regular orbits (blue, purple) in the magnetic periodic Sinai billiard (MPSB) with disc radius $r$.

in billiards, including rigorous proofs of their existence, have been studied in the literature and can, e.g., be found in Refs. 20-22 and references therein.

Quantitative studies of the Lyapunov exponent in actual physical billiards are surprisingly rare, however. A computational framework for calculating $\lambda$ in billiard systems was formulated by Dellago, Posch, and Hoover in Refs. 23 and 24 (to which we refer to as the "DPH framework" in the following, and which we will extend to the dynamics in magnetic fields). Alternative approaches are presented, e.g., in Refs. 25 and 26. In the literature, Lyapunov exponents have been computed for the PSB on square ${ }^{27}$ and hexagonal ${ }^{23,27}$ lattices, as well as for the stadium billiard, ${ }^{23,28}$ which is related to the mushroom billiard. Furthermore, there are results for the magnetic elliptical billiard ${ }^{29}$ and the inverse magnetic stadium. ${ }^{30}$

All these quantitative calculations rely on detailed numerical simulations of the complex billiard dynamics. In this paper, however, we want to follow a different approach exploring approximate expressions for the parameter dependence of the Lyapunov exponents in some paradigmatic cases, especially of billiards with the "mixed" phase space, where regions of regular and chaotic dynamics coexist. Our work is motivated by a recent study that has shown that magnetoresistance measurements in graphene and semiconductor nanostructures directly reflect the parameter dependence of the chaotic phase space volume. ${ }^{9}$ This is due to the fact that characteristic transport times in the chaotic sea are fundamentally linked to the respective volume of the chaotic phase space in the corresponding billiards. In particular, for the magnetic periodic Sinai billiard (MPSB) in Fig. 1(b), it was analytically shown that the mean collision time $\kappa(B)$ between successive collisions with the discs (of radius $r$ ) as a function of an applied external magnetic field $B$ is equal to the varying chaotic phase space portion $g_{c}(B)$ times the value of $\kappa$ at a zero magnetic field, ${ }^{9}$ i.e.,

$$
\kappa(B)=g_{c}(B) \times \kappa(0)=g_{c}(B) \times \frac{1-\pi r^{2}}{2 r} .
$$

[The value of $\kappa(0)$ is obtained from Eq. (3).] For the convenience of the reader, we replicate the proof of Eq. (1) from Ref. 9, which uses Kac's lemma, ${ }^{31-33}$ in Appendix D.

The Lyapunov exponent in billiards is also linked to mean collision times as the following back-of-the-envelope calculation motivates. Let us study the perturbation growth, i.e., the exponential growth of the phase space distance $|\delta \boldsymbol{\Gamma}(t)|$ of two initially infinitesimally close by trajectories. The origin of the exponential perturbation growth and thus of chaos in billiards is collisions with curved boundaries. ${ }^{34}$ Assuming an average perturbation growth increase of $C$ between collisions with curved boundaries and an average time $\kappa$ between such collisions, one would expect a perturbation growth of $|\delta \boldsymbol{\Gamma}(t)| \approx C^{t / \kappa}|\delta \boldsymbol{\Gamma}(0)|$. This means for the Lyapunov exponent of the ergodic component of phase space [see the definition in Eq. (4)], we expect

$$
\lambda \approx \frac{\log (C)}{\kappa} .
$$

In general, billiards have noncurved boundaries as well as curved ones. The mean collision time $\tau$ between two consecutive collisions with any parts of the billiard boundary is known analytically for "any" billiard and is given by

$$
\tau=\frac{\pi|Q|}{|\partial Q|}
$$

where $|Q|$ is the area of the billiard and $|\partial Q|$ is the total length of its boundary. ${ }^{20}$ Since Eq. (3) is averaged over the entire boundary of the billiard, it includes contributions from both chaotic and regular components (if any). Also, notice that a formula similar to Eq. (3) exists for billiards of any dimension, see Ref. 20 .

The starting point of our work is the observation that in billiards the mean collision time between curved boundaries $\kappa$ (a more precise definition will be given in Sec. II C) is fundamentally linked to the chaotic phase space volume $V_{\mathrm{CH}}$ by Kac's lemma. ${ }^{31-33}$ Therefore, the Lyapunov exponent is also linked to the chaotic phase space volume, and the aim of this paper is to explore how far considerations like Eq. (1) allow us to estimate the parameter dependence of the Lyapunov exponent in billiard systems. To this end, we will analyze the contributions to the approximate expression (2) and compare it with detailed numerical simulations. In Sec. II, we provide the basic framework we will use for computing $\lambda$, as well as apply the aforementioned back-of-the-envelope calculation to realistic perturbation growth. Following in Sec. III, we present our results about the periodic Sinai billiard and the mushroom billiard. We conclude by discussing the generality of our approach, while presenting one additional billiard with mixed phase space, the inverse stadium billiard, which has been studied by Vörös et al. ${ }^{30}$

\section{LYAPUNOV EXPONENTS IN BILLIARDS}

In this section, we first give a brief overview of the DPH framework $^{23,24}$ for numerically computing $\lambda$, reciting the equations that will be relevant for our study. We will then extend the framework to motion in an external magnetic field. In the following, we will assume that the point particle in the billiard has unit mass, and momentum and velocity are the same.

The (maximum) Lyapunov exponent is defined based on the evolution of the four-dimensional perturbation vector $\delta \boldsymbol{\Gamma}=(\delta \mathbf{q}, \delta \mathbf{p})^{T}$ as

$$
\lambda_{\boldsymbol{\Gamma}(0), \delta \boldsymbol{\Gamma}(0)}=\lim _{t \rightarrow \infty} \frac{1}{t} \log \frac{|\delta \boldsymbol{\Gamma}(t)|}{|\delta \boldsymbol{\Gamma}(0)|},
$$


with $\delta \boldsymbol{\Gamma}$ evolving according to the evolution equations in tangent space, $\delta \dot{\boldsymbol{\Gamma}}=J(\boldsymbol{\Gamma}(\mathbf{t})) \cdot \delta \boldsymbol{\Gamma}$, where $J$ is the Jacobian matrix of the equations of motion. For almost all $\boldsymbol{\Gamma}(0)$ inside an ergodic component of phase space, the value of $\lambda$ does not depend on the initial condition.

\section{A. Without magnetic field}

The time evolution in the tangent space for a particle moving in a straight line is

$$
\delta \boldsymbol{\Gamma}(t)=\left(\begin{array}{c|c}
\mathbb{I}_{2 \times 2} & t \cdot \mathbb{I}_{2 \times 2} \\
0_{2 \times 2} & \mathbb{I}_{2 \times 2}
\end{array}\right) \delta \boldsymbol{\Gamma}(0) .
$$

At discrete time points, Eq. (5) is interrupted by collisions with the boundary and the perturbation vector changes discontinuously. The perturbation vector just after the collision (we will use' to label quantities right after the collision) is derived from the one just before the collision as $^{24}$

$$
\delta \boldsymbol{\Gamma}^{\prime}=\left(\begin{array}{l}
\delta \mathbf{q}-2(\delta \mathbf{q} \cdot \mathbf{n}) \mathbf{n} \\
\delta \mathbf{p}-2(\delta \mathbf{p} \cdot \mathbf{n}) \mathbf{n}-2 \gamma_{r} \frac{(\delta \mathbf{q} \cdot \mathbf{e})}{\cos \phi} \mathbf{e}^{\prime}
\end{array}\right)
$$

for a collision with a boundary segment of curvature $\gamma_{r}$. The two types of boundaries we will encounter in this work are straight walls and the boundaries of circular discs. For a straight wall section $\gamma_{r}=0$ and for a disc of radius $r$, we have $\gamma_{r}= \pm \frac{1}{r}$, with-for collisions happening from the inside of the disc (as in the $\mathrm{MB}$ ) and + otherwise (as in the PSB). Here, $\mathbf{n}$ denotes the normal vector of the boundary segment at the collision point $\mathbf{q}$, and $\phi$ is the angle of incidence (measured with respect to the normal to the surface). The vectors $\mathbf{e}$ and $\mathbf{e}^{\prime}$ are unit vectors orthogonal to the incident and reflected momenta $\mathbf{p}$ and $\mathbf{p}^{\prime}$, respectively (for more details, see Ref. 24).

Notice that a collision with a straight wall does not change the norm of $\delta \boldsymbol{\Gamma}$ because both the coordinate and the velocity components are reflected specularly.

\section{B. With magnetic field}

We now extend the DPH formalism for a particle experiencing a magnetic field perpendicular to the billiard plane. In this section, we present only the final expressions. The full calculations are presented in Appendix A.

The magnetic field is uniform, with value $B$ (positive means a counterclockwise rotation). The free evolution of the perturbation vector $\delta \boldsymbol{\Gamma}(t)$ in the presence of a perpendicular magnetic field is given by

$$
\begin{aligned}
\delta \boldsymbol{\Gamma}(t) & =\mathbb{B} \cdot \delta \boldsymbol{\Gamma}\left(t_{0}\right), \\
\mathbb{B} & =\left(\begin{array}{c|cc}
\mathbb{I}_{2 \times 2} & \begin{array}{c}
\rho \sin (\omega t) \\
-\rho(\cos (\omega t)-1)
\end{array} & \rho(\cos (\omega t)-1) \\
\hline 0_{2 \times 2} & \begin{array}{l}
\cos (\omega t) \\
\sin (\omega t)
\end{array} & -\sin (\omega t) \\
\hline & \cos (\omega t)
\end{array}\right),
\end{aligned}
$$

with the cyclotron frequency $\omega=2 B$ and the cyclotron radius $\rho=1 / \omega$. As already mentioned in the introduction, in the billiard, the particle always moves with unit velocity by convention. The expressions that give the discontinuous change of the perturbation vector at a collision with a wall or disc are

$$
\begin{aligned}
\delta \boldsymbol{\Gamma}^{\prime} & =\left(\begin{array}{c}
\delta \mathbf{q}-2(\delta \mathbf{q} \cdot \mathbf{n}) \mathbf{n} \\
\delta \mathbf{p}-2(\delta \mathbf{p} \cdot \mathbf{n}) \mathbf{n}-2 \gamma_{r} \frac{\langle\delta \mathbf{q}, \mathbf{e}\rangle}{\cos \phi} \mathbf{e}^{\prime}
\end{array}\right)-\omega \frac{(\delta \mathbf{q} \cdot \mathbf{n})}{(\mathbf{p} \cdot \mathbf{n})}\left(\begin{array}{c}
0 \\
\mathbb{S} \cdot \mathbf{p}
\end{array}\right), \\
\mathbb{S} & =2\left(\begin{array}{cc}
-2 n_{1} n_{2} & n_{1}^{2}-n_{2}^{2} \\
n_{1}^{2}-n_{2}^{2} & 2 n_{1} n_{2}
\end{array}\right), \quad \mathbf{n}=\left(\begin{array}{l}
n_{1} \\
n_{2}
\end{array}\right),
\end{aligned}
$$

where $\gamma_{r}$ again is the curvature of the wall segment ( 0 for a straight wall, $\pm \frac{1}{r}$ for a disc).

\section{The "toy model"}

Before finding an approximate expression for the value of $\lambda$ in our model systems, it is worthwhile to get an impression of how the norm of the perturbation vector evolves with time. In Fig. 2, we show typical plots for the three different billiards. We computed the perturbation growth using the DPH framework, sampling the perturbation vector immediately before and after every collision to resolve the instantaneous jumps. As the DPH evolution is linear, in the actual numerical simulations, we renormalized the perturbation vector after sampling to prevent numerical errors due to the rapid perturbation growth.

Let us first examine Figs. 2(a) and 2(b). We see that the norm of the perturbation vector changes in two ways. Let the $j$ th collision with a disc happen at time $t_{j}=\sum_{i=0}^{j-1} \Delta t_{i}=t_{j-1}+\Delta t_{j-1}$. There a discontinuous change of the perturbation vector norm happens, so that $\left|\delta \boldsymbol{\Gamma}_{j}^{\prime}\right|=a_{j}\left|\delta \boldsymbol{\Gamma}_{j}\right|$ (in general, $a_{j}$ is a function of $\delta \boldsymbol{\Gamma}_{j}$ ). The collision event is followed by a time-interval $\Delta t_{j}$, in which the perturbation norm changes continuously because there are no collisions with curved boundaries. Just before the next collision with a disc the perturbation norm takes the value $\left|\delta \boldsymbol{\Gamma}_{j+1}\right|$. In the following, we will refer to these repeated segments of the growth curve as "elementary growth segments," starting with one collision event with a disc and ending just before the next one. In general, it is the segment of the perturbation growth curve between successive dispersing or defocussing collisions which are the origin of chaos in billiards. ${ }^{34}$ An elementary growth segment thus reflects the perturbation growth during an "effective free path" as defined by Bunimovich. ${ }^{3}$

A crucial simplification we do in deriving an approximate expression for the Lyapunov exponent will be to express the perturbation growth in the time-interval $\Delta t_{j}$ as a function $z\left(\Delta t_{j}\right)$ of the interval length. The actual precise value $\left|\delta \boldsymbol{\Gamma}_{j+1} / \delta \boldsymbol{\Gamma}_{j}^{\prime}\right|$ is not a simple scalar function of the time interval since, for example, in the case of the PSB we can obtain from Eq. (5)

$$
\left|\delta \boldsymbol{\Gamma}_{j+1}(\Delta t)\right|=\sqrt{\left(\delta \mathbf{q}_{j}^{\prime}+\delta \mathbf{p}_{j}^{\prime} \Delta t\right)^{2}+\left(\delta \mathbf{p}_{j}^{\prime}\right)^{2}}
$$

[due to the linearity of the equations of motion of the tangent space we can assume a norm of $\left|\delta \boldsymbol{\Gamma}_{j}^{\prime}\right|=1$ in Eq. (9)]. Equation (9) depends on the initial orientations of both the momentum and position deviation vectors and thus is not a function of just $\Delta t$.

We will show, however, by analyzing numerical data, that a reasonable approximation can be obtained by assuming that such a function $z(\Delta t)$ exists. Notice that this assumption is only regarding the existence of $z$. The functional form and its complexity can be completely arbitrary (and in fact in the following we have three different versions of $z$ for the different billiards). For each elementary 

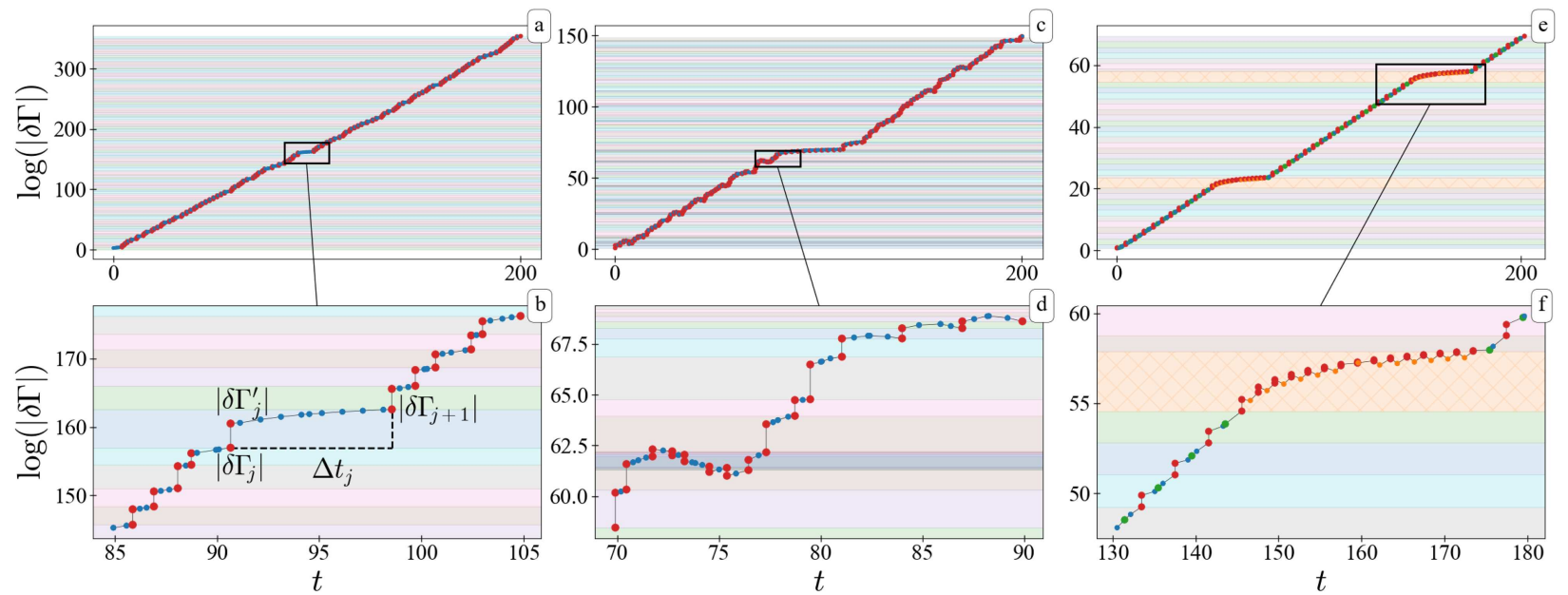

FIG. 2. Typical time evolution of the logarithm of the norm of the perturbation vector $|\delta \boldsymbol{\Gamma}(t)|$ for the periodic Sinai billiard without (a) and with (c) magnetic field $(B=1)$ and for the mushroom billiard (e) (without magnetic field). Zoom-ins are below each panel. For (a)-(d), red markers mean collision with the disc, while blue markers mean "collision" with the periodic walls (not a true collision, but a way of recording the value of $|\delta \Gamma|)$. For (e)-(f), red is collision with cap head, orange with cap walls, blue with stem sides, and green with stem bottom. The colored background stripes denote the "elementary growth segments" discussed in Sec. II C (random colors are used) with hatched orange color used for the laminar episodes (see Sec. II C).

growth segment, we thus write

$$
\left|\delta \boldsymbol{\Gamma}_{j+1}\right|=z\left(\Delta t_{j}\right) \times a_{j} \times\left|\delta \boldsymbol{\Gamma}_{j}\right|=z\left(\Delta t_{j}\right) \times\left|\delta \boldsymbol{\Gamma}_{j}^{\prime}\right| .
$$

We then recursively apply Eq. (10) to get

$$
\begin{aligned}
\left|\delta \boldsymbol{\Gamma}_{n}\right| & =\prod_{i=0}^{n-1} a_{i} z\left(\Delta t_{i}\right)|\delta \boldsymbol{\Gamma}(0)| \quad \Rightarrow \\
\log \left(\left|\delta \boldsymbol{\Gamma}_{n}\right|\right) & =\sum_{i=0}^{n-1} \log \left(a_{i}\right)+\log \left(z\left(\Delta t_{i}\right)\right)
\end{aligned}
$$

(using $|\delta \boldsymbol{\Gamma}(0)|=1$ ) and with $T_{n}=\sum_{i=0}^{n-1} \Delta t_{i}$ we use Eq. (4) to write $\lambda=\lim _{n \rightarrow \infty} \log \left(\left|\delta \boldsymbol{\Gamma}_{n}\right|\right) / T_{n}$. The quotient of the infinite sums is the same as the ratio of the average over all unit cells (denoted by $\langle\cdot\rangle$ ), i.e.,

$$
\begin{aligned}
\lambda & \approx \frac{1}{\kappa}(\langle\log (a)\rangle+\langle\log (z(\Delta t))\rangle), \\
\kappa & \equiv\langle\Delta t\rangle .
\end{aligned}
$$

Averaging over the unit cells implicitly assumes the ergodicity. Notice also that in some billiards, there could be several ergodic chaotic components that are separated from each other. In such cases, the above process has to be applied to each component separately, since each component has its own exponent $\lambda$. For the billiards considered here, we have found that furthermore $\langle\log (z(\Delta t))\rangle$ $=\log (z(\langle\Delta t\rangle))=\log (z(\kappa))$ is good approximation that we will use. This approximation is valid when the standard deviation of $\Delta t$ is small (compared to its mean).

In the remainder of the text, we will call Eq. (12) the "toy model." It is the more detailed version of the back-of-the-envelope calculation given in the introduction. In Sec. III we will apply this toy model to specific billiards and see how well it approximates the Lyapunov exponent and its parameter dependence.

\section{Software}

All numerical computations presented in this paper were performed with an open source software to simulate billiards, "DynamicalBilliards.jl." ${ }^{55}$ In this paper, we extend the DPH framework to magnetic fields. We also implemented this extension in the software (which previously only included the nonmagnetic case). All code we used for this paper, including all code to replicate the figures we show here, is publicly available on GitHub: https://github.com/Datseris/arXiv' 1904.05108.

\section{RESULTS}

\section{A. Periodic Sinai billiard}

We start our analysis with the PSB because it is the simplest case and we are able to give a fully analytical expression for the Lyapunov exponent in the simplified toy model. We note that in the absence of a magnetic field, the PSB is ergodic and its phase space is not mixed. Nevertheless, it will serve as a pedagogic example of how the toy model approximates the Lyapunov exponent.

For the PSB, $\tau=\kappa$ and the value of $\tau$ is known from Eq. (3)

$$
\kappa_{\mathrm{PSB}}=\frac{1-\pi r^{2}}{2 r} \text {. }
$$

An approximation for $z(\Delta t)$ is easily found as well from Eq. (9), namely,

$$
z_{\mathrm{PSB}}(t) \approx \sqrt{1+t^{2}}
$$



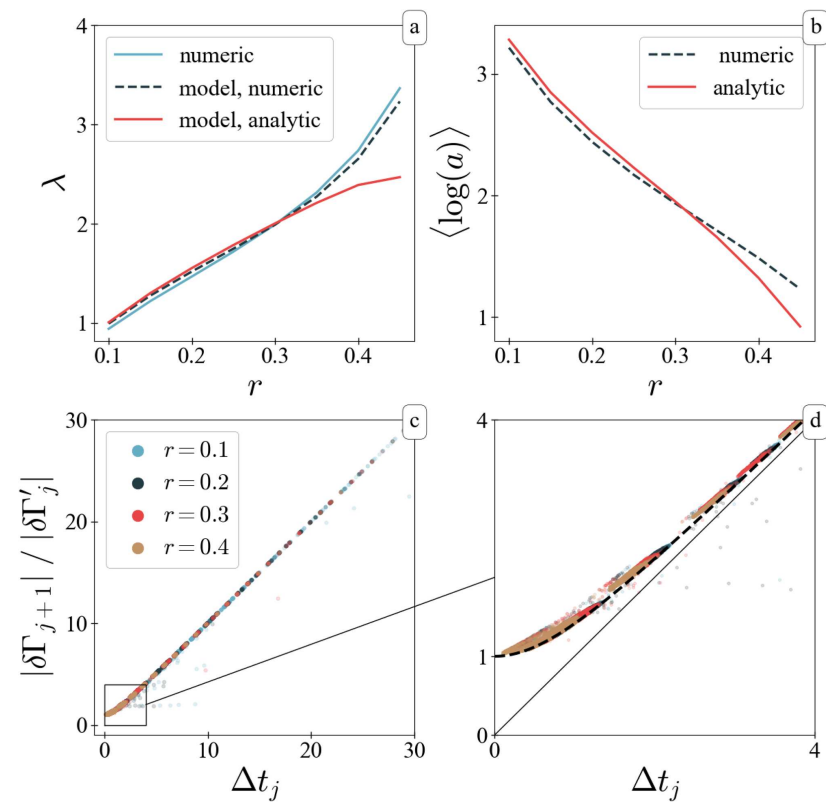

FIG. 3. (a) Lyapunov exponent of the periodic Sinai billiard for different radii, compared with the toy model. The dashed curve obtains $\langle\log (a)\rangle$ by numeric average, while the red curve uses Eq. (19). The blue curve is using the DPH framework. (b) Average value of $\langle\log (a)\rangle$ used in panel (a). (c) Perturbation norm increases during the free flight part. In the zoom in (d), we also plot the curve $\sqrt{1+\Delta t^{2}}$ as a dashed line.

which uses the assumption that after a collision with a disc, the momentum contribution to the perturbation vector is much larger than the position contribution, i.e., $\left|\delta \mathbf{p}^{\prime}\right| \gg\left|\delta \mathbf{q}^{\prime}\right|$. Numerical calculations show that this approximation is valid for small enough radii (see Fig. 3). The instantaneous change factor $\langle\log (a)\rangle$ is rather large for all but very large disc radii. Also, as seen in panel (c) and its inset (d), Eq. (15) almost perfectly approximates the perturbation norm increase during the free flight part.

We still need an approximate expression for $a$, the instantaneous change factor, which we can derive from Eq. (6). Since the norm of the position deviation does not change at the collision, we focus on the momentum deviation $\delta \mathbf{p}^{\prime}=\delta \mathbf{p}^{(r)}-\frac{2}{r} \frac{\delta \mathbf{q} \cdot \mathbf{e}}{\cos (\phi)} \mathbf{e}^{\prime}$ with $\delta \mathbf{p}^{(r)}=\delta \mathbf{p}-$ $2(\delta \mathbf{p} \cdot \mathbf{n}) \mathbf{n}$ (if not explicitly written otherwise all quantities in this paragraph have a collision-time index $j$, which we suppress to make the symbols simpler). We define $A=\frac{2}{r} \frac{\delta q \cdot \mathbf{e}}{\cos (\phi)}$ and carrying out the calculations leads to

$$
\left|\delta \boldsymbol{\Gamma}^{\prime}\right| /|\delta \boldsymbol{\Gamma}|=a=\sqrt{1+A^{2}-2 A\left(\mathbf{e}^{\prime} \cdot \delta \mathbf{p}\right)}
$$

again using the assumption that $\left|\delta \boldsymbol{\Gamma}_{j}\right|=1$ (and recall that $\left|\delta \mathbf{p}^{(r)}\right|$ $\left.=|\delta \mathbf{p}|,\left|\delta \mathbf{q}^{\prime}\right|=|\delta \mathbf{q}|\right)$.

We now need to average $\langle\log (a)\rangle$. We start our approximation by replacing the inner product $(\delta \mathbf{q} \cdot \mathbf{e})$ by an averaged quantity $b(r)$ (we show below how $b$ depends on $r$ ). It is expected that perturbations will grow and orient themselves perpendicular to the particle's direction of motion. Since $\mathbf{e}$ is a unit vector perpendicular to the particle's momentum and thus parallel to $\delta \mathbf{q}$, this means that $\langle|(\delta \mathbf{q} \cdot \mathbf{e})| /|\delta \boldsymbol{\Gamma}|\rangle=\langle|\delta \mathbf{q}| /|\delta \boldsymbol{\Gamma}|\rangle=b(r)$.

Which portion of $|\delta \boldsymbol{\Gamma}|$ is contained in $|\delta \mathbf{q}|$ is answered based on how perturbations evolve during the free flight part. Starting from the $j$ th collision the perturbations evolve for time $\Delta t_{j}$. Using Eq. (9) (and assuming that the cross terms $\delta \mathbf{p} \cdot \delta \mathbf{q}$ will drop out in the averaging), we obtain

$$
\frac{\left|\delta \mathbf{q}_{j+1}\right|}{\left|\delta \boldsymbol{\Gamma}_{j+1}\right|}=\frac{\sqrt{\frac{\left|\delta \mathbf{q}_{j}^{\prime}\right|^{2}}{\left|\delta \mathbf{p}_{j}^{\prime}\right|^{2}}+\Delta t_{j}^{2}}}{\sqrt{\frac{\left|\delta \mathbf{q}_{\mathbf{j}}^{\prime}\right|^{2}}{\left|\delta \mathbf{p}_{j}^{\prime}\right|^{2}}+1+\Delta t_{j}^{2}}} .
$$

To analytically resolve Eq. (17), we use the same assumption as in Eq. (15), $\left|\delta \mathbf{p}^{\prime}\right| \gg\left|\delta \mathbf{q}^{\prime}\right|$. We then average, replacing $\Delta t$ by $\kappa$, which leads to

$$
b(r)=\sqrt{\frac{\kappa_{\mathrm{PSB}}^{2}}{1+\kappa_{\mathrm{PSB}}^{2}} .}
$$

We discard the term $2 A\left(\mathbf{e}^{\prime} \cdot \delta \mathbf{p}\right)$ in Eq. (16) again assuming that the inner product averages to 0 . Now, the only variable left to average over is $\phi$, the angle with respect to the normal vector. This is distributed in $[-\pi / 2, \pi / 2]$ with probability distribution of $\cos (\phi) / 2$. Therefore,

$$
\begin{aligned}
\langle\log (a)\rangle & =\int_{-\frac{\pi}{2}}^{\frac{\pi}{2}} \log \left(\sqrt{1+\left(\frac{2 b}{r \cos (\phi)}\right)^{2}}\right) \frac{\cos (\phi)}{2} d \phi \\
& =\frac{\operatorname{csch}^{-1}\left(\frac{2 b}{r}\right) \sqrt{4 b^{2}+r^{2}}}{r}+\log \left(\frac{b}{r}\right),
\end{aligned}
$$

with $\operatorname{csch}^{-1}$ being the inverse hyperbolic cosecant.

We then put Eqs. (14), (15), (18), and (19) into the toy model of Eq. (12) and obtain an analytic approximation for the Lyapunov exponent

$$
\begin{aligned}
\lambda_{\mathrm{PSB}}(r)= & \frac{2 r}{1-\pi r^{2}}\left(\frac{\operatorname{csch}^{-1}\left(\frac{2 b(r)}{r}\right) \sqrt{4 b(r)^{2}+r^{2}}}{r}\right. \\
& \left.+\log \left(\frac{b(r)}{r}\right)+\log \left(\sqrt{1+\left(\frac{1-\pi r^{2}}{2 r}\right)^{2}}\right)\right) .
\end{aligned}
$$

The result is shown in Fig. 3(a), compared with the numerical value of $\lambda$ using the DPH framework as well as with the result of computing the term $\langle\log (a)\rangle$ in the toy model numerically from the evolution of the perturbation vector norm. All three curves are in excellent agreement for small and intermediate $r$, only for large $r$ does Eq. (20) slightly deviate from the numerical values because the approximation for $b(r)$ in Eq. (18) and thus for $\langle\log (a)\rangle$ becomes less accurate.

\section{B. Magnetic periodic Sinai billiard}

We now want to apply the same process to the MPSB, which, however, has a mixed phase space: there exist collisionless orbits like those seen in Fig. 1(b) that constitute the regular part of phase space (other unstable periodic orbits of zero measure are not relevant here). We are of course only considering the Lyapunov exponent of the 
chaotic part of the phase space, which means that we initialize particles only in the chaotic phase space region. The mean collision time $\kappa_{\mathrm{MPSB}}$ between successive collisions with discs is also only defined for the chaotic phase space part (as the regular trajectories do not collide with the discs).

The free flight evolution in the MPSB is fundamentally different from the PSB. Not only are the functional forms different but in addition due to magnetic focusing, it is possible (and in fact quite frequent) for the perturbation norm to "decrease" during the evolution, as can be seen in Figs. 4(e) and 4(f). In addition, as visible in Fig. 2(d), it is also possible for the norm to decrease during the instantaneous change as well.

This more complex behavior is of course hidden in the more complicated formulas of our extension to the DPH framework for magnetic fields. For example, explicitly writing out Eq. (7) gives

$$
\begin{aligned}
|\delta \boldsymbol{\Gamma}(t)|= & {\left[\left(\delta p_{x}^{2}+\delta p_{y}^{2}\right)\right.} \\
& +\left(\delta q_{x}+\delta p_{y} \frac{\cos (\omega t)-1}{\omega}+\delta p_{x} \frac{\sin (\omega t)}{\omega}\right)^{2} \\
& \left.+\left(\delta q_{y}-\delta p_{x} \frac{\cos (\omega t)-1}{\omega}+\delta p_{y} \frac{\sin (\omega t)}{\omega}\right)^{2}\right]^{1 / 2}
\end{aligned}
$$

(where again we assumed $|\delta \boldsymbol{\Gamma}(0)|=1$ ). The consequences of Eq. (21) can be seen in Figs. 4(d) and 4(e). Using a univariate scalar function $z(\Delta t)$ to approximate these distributions appears to be a bold move, but in the end it will turn out to give good approximation. To obtain $z(\Delta t)$, we simplify Eq. (21) to

$$
z_{\mathrm{MPSB}}(t)=\sqrt{1+\left(\frac{1-\cos (\omega t)}{\omega}\right)^{2}+\left(\frac{\sin (\omega t)}{\omega}\right)^{2}},
$$

which is also plotted in Figs. 4(d) and 4(e).

In the next step, we compute $\langle\log (a)\rangle$ numerically and use its value in the toy model along with $z_{\mathrm{MPSB}}(\kappa(B))$. We remind that the value of $\kappa$, the mean collision time between discs in MPSB, is not known analytically but it is connected with the chaotic phase space portion through Eq. (1). The results of the toy model are presented in Fig. 4.

Besides the fact that our toy model approximates $\lambda$ very accurately, Fig. 4 shows the impact of phase space restriction on $\lambda$. In our toy model, the value of $\lambda$ is composed of five contributions, the first being the denominator $\kappa$. The value of $\langle\log (a)\rangle$ itself has two contributions, one again stemming from $\kappa$ (as shown in Sec. III A) and the other from $B$. The function $z_{\text {MPSB }}$ also has two contributions, one from $B$ and one from $\kappa$. Therefore, three out of five contributions to $\lambda$ are inherently linked to the restriction of the chaotic phase space by regular orbits.

\section{Mushroom billiard}

Because the volume fractions of the regular and the chaotic phase space regions are not known analytically in the MPSB, we have turned to a billiard that also has a mixed phase space but allows us to calculate these fractions, and, as we will show, the relevant average time scales analytically: the mushroom billiard (MB). The regular orbits in the $\mathrm{MB}$ are orbits forever staying in the cap, evolving exactly
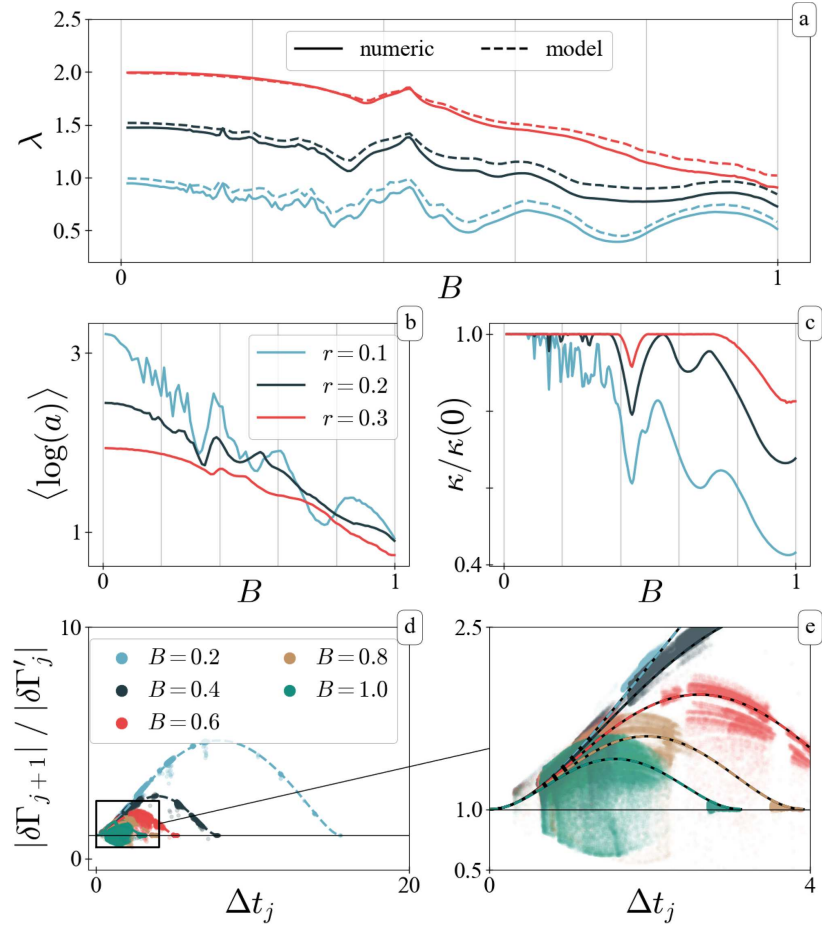

FIG. 4. (a) Lyapunov exponent of the magnetic periodic Sinai billiard (MPSB) for different radii vs magnetic field, compared with the toy model. The solid curves are the numeric result $\lambda$, the dashed curves are the toy model using the numeric average of $\langle\log (a)\rangle$. (b) Numeric average of $\langle\log (a)\rangle$ vs the magnetic field. (c) Chaotic phase space portion $\left[g_{c}=\kappa / \kappa(0)\right]$ of the MPSB [(a)-(c) share legend]. (d) and (e) Perturbation norm change during the free flight in the MPSB (calculated with the DPH framework). Plotted with dashed lines are Eq. (22) (data for $r=0.2$ ).

like they would as if they were in a circular billiard. ${ }^{19}$ The tangential circle to these orbits has a radius $\geq w / 2$, as shown in Fig. 1(a). The rest of the orbits, which do not satisfy this criterion, eventually enter the stem and are chaotic. The tangential circle argument was used in Ref. 5 to obtain an analytic expression for the regular phase space volume $V_{\mathrm{REG}}$ of the $\mathrm{MB}$ as a function of the billiard parameters,

$$
\begin{aligned}
V_{\mathrm{REG}} & =2 \pi\left(\arccos \left(\frac{w}{2}\right)-\frac{w}{2}\left(1-\frac{w^{2}}{4}\right)\right), \\
V_{\mathrm{TOT}} & =2 \pi(h w+\pi / 2), \\
V_{\mathrm{CH}} & =V_{\mathrm{TOT}}-V_{\mathrm{REG}},
\end{aligned}
$$

where $V_{\text {TOT }}$ and $V_{\mathrm{CH}}$ are the total and chaotic phase space volume (all lengths are scaled to the cap radius $r$ which is fixed to $r=1$ ). The parameter dependence of $V_{\mathrm{CH}}$ is illustrated in Figs. 5(c) and 5(d). Interestingly, $V_{\mathrm{CH}}$ does not vanish for small $h$, although it is obvious that there are no chaotic orbits for $h=0$. This discontinuity is due to the fact that the volume of chaotic phase space in the cap is independent of stem height for nonzero $h$, but drops to zero for $h=0$. 

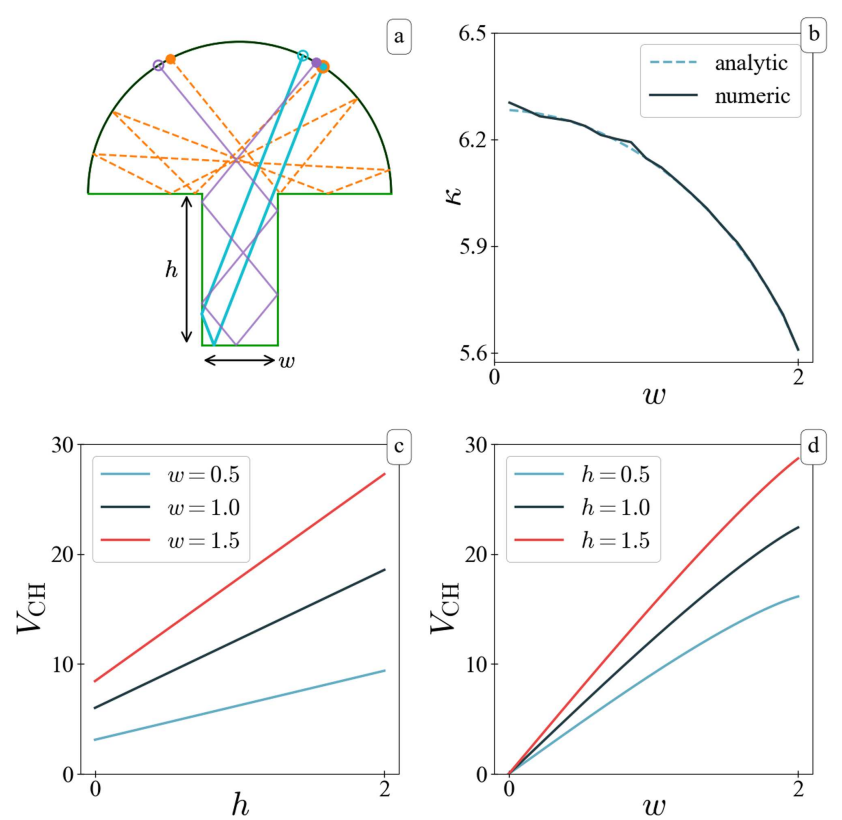

FIG. 5. (a) A laminar episode (orange, dashed) and two chaotic episodes (blue purple) in the mushroom billiard. Start and end of each episode are denoted with closed and open circles and the blue episode starts directly after the orange. The cap head is plotted in dark color to differentiate. (b) Mean return time to the stem bottom, which is equivalent with the average elementary growth segment time, Eqs. (27) and (28). (c) and (d) Volume of chaotic phase space for the mushroom billiard.

In Figs. 6(c) and 6(d), we present a scatterplot of various possible increases of the perturbation norm during the unit cells. We found that there are clearly distinct contributions to the increase, each seemingly approximated as a linear function of $\Delta t$. By analyzing the dynamics in more detail, it turns out that the different contributions of Fig. 6(c) and 6(d) come from the trapping of the chaotic orbits in the regular phase space. In the coordinate space, this means that the particles get trapped in the cap and mimic the motion of the regular phase space there until eventually escaping after some time. This effect is often called "intermittency" and is known to occur in mushroom billiards. ${ }^{36,37}$ Intermittent behavior in the MB can happen in the stem as well, where orbits stay trapped bouncing between the stem walls.

We, therefore, have to separate the elementary growth segment into two different "episodes:" the chaotic episode $c$ and the laminar episode $\ell$, where the particle is trapped in the cap. Notice that the second intermittent behavior, trapping in the stem, does not lead to a new type of dynamics, but is just prolonged chaotic episodes (similarly to a large free flight in the PSB). We show the two episodes in Fig. 5(a). Numeric calculations shown in Figs. 6(e) and 6(f) show that each episode has a different average time, $\tau_{c}, \tau_{\ell}$, respectively.

During the chaotic episodes, the picture is very similar to the PSB. A collision with the cap head gives an instantaneous increase to $|\delta \boldsymbol{\Gamma}|$, followed by an approximately linear increase until the next collision with the cap head. Here, the linear increase approximation is valid because for the chaotic episodes $\Delta t \gtrsim 2 h+2-w$. After colliding with the cap head, the particle may return to the stem immediately which initializes another chaotic episode. Occasionally, after ending a chaotic episode, the particle will get trapped in the cap [see Fig. 5(a), orange], starting a laminar episode. Even though there are successive collisions with the cap head in this episode, the perturbations do not increase exponentially. The successive instantaneous increases are very quickly becoming insignificant [see Figs. 2(e) and $2(\mathrm{f})$ ] due to the fact that cap collisions have an initially focusing effect which only becomes defocusing if the consecutive free motion is long enough, which is not the case in the laminar episodes. Therefore, the overall perturbation growth inside the cap trapping episodes is "linear."

Let $n_{c}$ and $n_{\ell}$ be the counts of chaotic and laminar episodes up to time $T$. Notice that $n_{\ell}$ is strictly less than $n_{c}$ since a chaotic episode always follows a laminar episode, but the inverse is only occasionally true. In the limit $T \rightarrow \infty$, we define $f_{\ell}=n_{\ell} /\left(n_{\ell}+n_{c}\right)$ to be the frequency of the laminar episodes. We then write the function $z$ as

$$
z_{\mathrm{MB}}(\Delta t)=\left(o_{c}+s_{c} \Delta t_{c}\right)+f_{\ell}\left(o_{\ell}+s_{\ell} \Delta t_{\ell}\right)
$$

with $o_{i}$ being the offset and $s_{i}$ being the slope of the linear approximation [we obtain these values with least squares fit to Fig. 6(c) and 6(d)]. For the chaotic episodes $s, o$ are constant vs $h, w$ while for the laminar episodes $o$ depends strongly on $w$. Also, for the chaotic episodes, $o$ has a negative value (of around -0.8 ) which is expected due to the focusing effect. We once again compute $\langle\log (z(\Delta t))\rangle$ simply by replacing $\Delta t$ by its average values $\tau_{c}, \tau_{\ell}$ in Eq. (26).

The instantaneous change factor $a$ is the same between the laminar and chaotic episodes so we do not need to separate it. Notice that for the laminar episode we only consider the first jump as the instantaneous increase. Subsequent jumps that decrease rapidly are encoded in the linear growth approximation. After computing $\langle\log (a)\rangle$ numerically, we still need a value for $\kappa_{\mathrm{MB}}$, the elementary growth segment average time, to apply our toy model $\lambda=\left(\langle\log (a)\rangle+\log \left(z_{\mathrm{MB}}\left(\tau_{c}, \tau_{\ell}\right)\right) / \kappa_{\mathrm{MB}}\right.$. Numerically, we can estimate

$$
\kappa_{\mathrm{MB}}=\tau_{c}+f_{\ell} \tau_{\ell}
$$

However, we can estimate $\kappa_{\mathrm{MB}}$ analytically as well, using Kac's lemma. ${ }^{31-33}$ The key to this is understanding that the mean elementary growth segment time is equivalent with the mean return time to the stem bottom, since all phases in the end of the day have to go there, since all phases are part of the chaotic phase space. given by

We present the full proof in Appendix B. The final expression is

$$
\kappa_{\mathrm{MB}}(h, w)=\frac{V_{\mathrm{CH}}(h, w)}{2 w} .
$$

We compare the analytic formula with the numeric result in Fig. 5(b) and find the expected perfect agreement, since Eq. (28) is exact. In Appendix $\mathrm{C}$, we also present an analytic approximation for $\tau_{c}$. Since we know $\kappa_{\mathrm{MB}}$ and $\tau_{c}$ analytically, we also know the product $f_{\ell} \tau_{\ell}$ (but we do not have an expression for $f_{\ell}$ or $\tau_{\ell}$ individually).

We can now use our toy model to compare with $\lambda$, which we do in Figs. 6(a) and 6(b). Again we find good agreement between the toy model and the numerical simulation using the DPH framework. The 

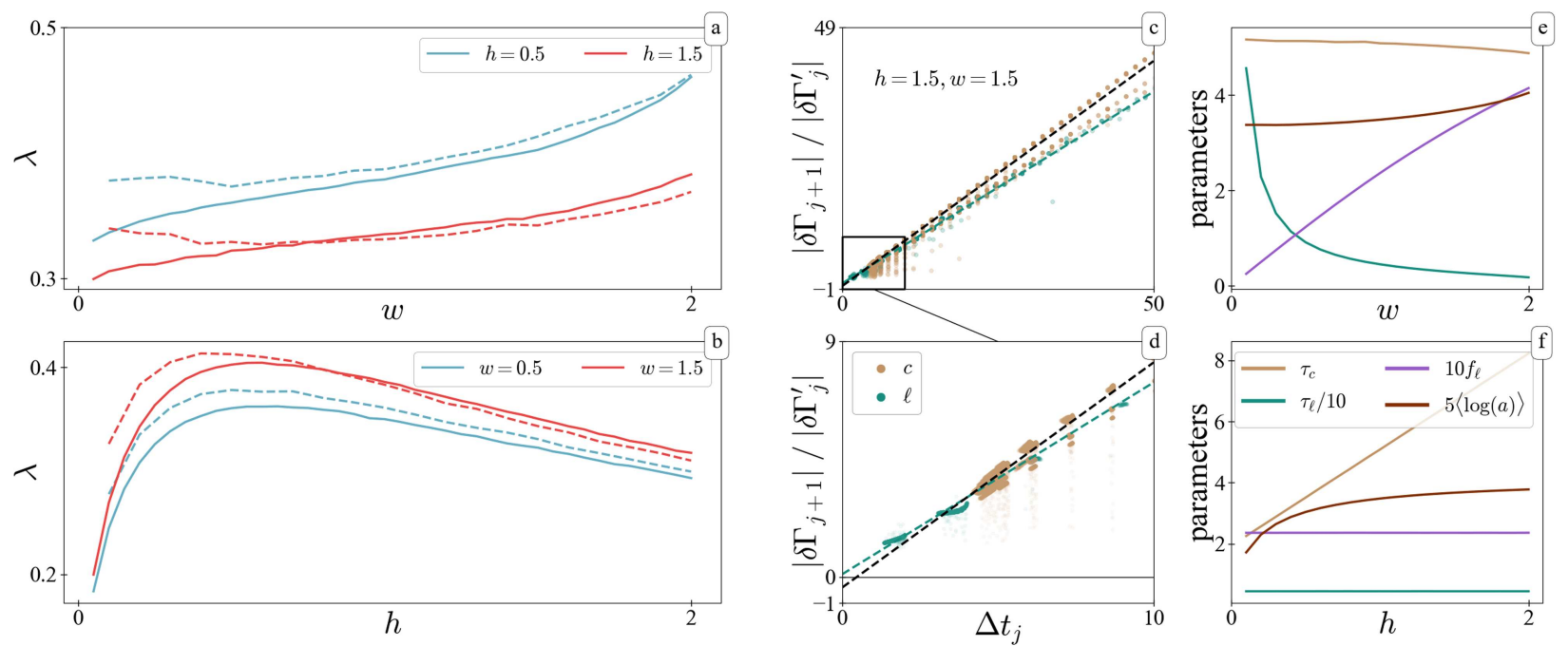

FIG. 6. (a) and (b) Lyapunov exponents in the mushroom billiard (MB) vs the width $w$ or height $h$ of the stem. Solid lines are numeric results using the DPH framework, and dashed lines are using the toy model. (c) and (d) Perturbation norm increases during the chaotic $c$ and laminar $\ell$ episodes. (e) and (f) Parameters of the toy model vs $w$ or $h$ (for constant $h=1$ and $w=1$, respectively); legend is shared.

model mildly diverges for very small $w$, probably because the mean laminar time $\tau_{\ell}$ diverges as seen in Fig. 6(e).

As was the case in the MPSB, the average elementary growth segment time $\kappa$ is inversely proportional to $\lambda$ and directly proportional to the chaotic phase space volume $V_{\mathrm{CH}}$. This shows that phase space restrictions have an immediate impact in the value of the Lyapunov exponent even for billiards with intermittent dynamics. Notice that in the MB both $V_{\mathrm{CH}}$ and $\lambda$ increase as $w$ increases. This is simply due to the dependence of $V_{\mathrm{CH}}$ on $w$, as well as the direct dependence of $\kappa_{\mathrm{MB}}$ on $1 / w$ [this, for example, was not the case in Eq. (1) for the MPSB].

\section{DISCUSSION}

To summarize, we have examined the value of the Lyapunov exponent $\lambda$ in chaotic billiards. We were able to create a conceptually simple model that approximates $\lambda$ very well. The model is based on how perturbations evolve in billiards "on average" and helps to understand how each part of the dynamics contributes to the perturbation increase. The simple model is written as Eq. (12), which is

$$
\lambda=\frac{1}{\kappa}(\langle\log (a)\rangle+\langle\log (z(\Delta t))\rangle),
$$

where $a$ the instantaneous change of $|\delta \boldsymbol{\Gamma}|$ at a collision with a curved boundary and $z(t)$ the continuous change of $|\delta \Gamma|$ in between collisions with curved boundaries. $\kappa$ is the average elementary growth segment time equal to the mean collision time between curved boundaries. The approximations that lead to the toy model were the following. First, we assumed that the chaotic phase space is ergodic and time averages can be replaced by phase space averages and that for $\Delta t, \log (a), \log (z(\Delta t))$, their averages are finite and greater than 0 . We then made the simplifying assumption that the norm of the perturbation vector increases continuously in between successive chaos-inducing collisions (i.e., in each elementary growth segment) as a "univariate" function of the time interval $z(\Delta t)$.

We used Eq. (12) to find an analytic expression for $\lambda$ in the periodic Sinai billiard (PSB). We have also shown that Eq. (12) can be used to analyze the Lyapunov exponent in the magnetic periodic Sinai billiard (MPSB), and by approximating the numerical curves identified the main contributions. We could follow the same approach for the mushroom billiard (MB), even though the process is complicated in this case by intermittent dynamics. In both billiards with mixed phase space, we connected the chaotic phase space volume with $\lambda$ through $\kappa$ and showed that $\lambda$ has a leading contribution given by the inverse of the chaotic phase space volume (for the MPSB, we used the chaotic "portion" instead of volume, because the total phase space volume does not depend on $B$ ).

To strengthen our point that a prominent contribution to the parameter dependence of the Lyapunov exponent in billiard is given by the inverse chaotic phase space volume, we present one final billiard, called the inverse stadium billiard (ISB) shown in Fig. 7(a), originally studied by Vörös et al. in Ref. 30. In this billiard, a particle is propagating inside the stadium on straight lines, but after passing the boundary of the stadium, it is subjected to a constant magnetic field, which brings the particle back inside the stadium, as depicted in Fig. 7(a). In the limit of infinite magnetic field, the ISB recovers the fully chaotic Bunimovich stadium, for finite magnetic fields it has a mixed phase space. Here, we do not want to analyze the ISB in any detail but only point out that also in this billiard the parameter dependence of its Lyapunov exponent is closely following the inverse of the chaotic phase space volume as shown in Figs. 7(b) and 7(c).

We want stress how different the mechanisms are that lead to chaos in the three different billiards. In the MPSB, it is dispersing as well as the magnetic field. In MB, it is defocusing, and, in the ISB, it is 


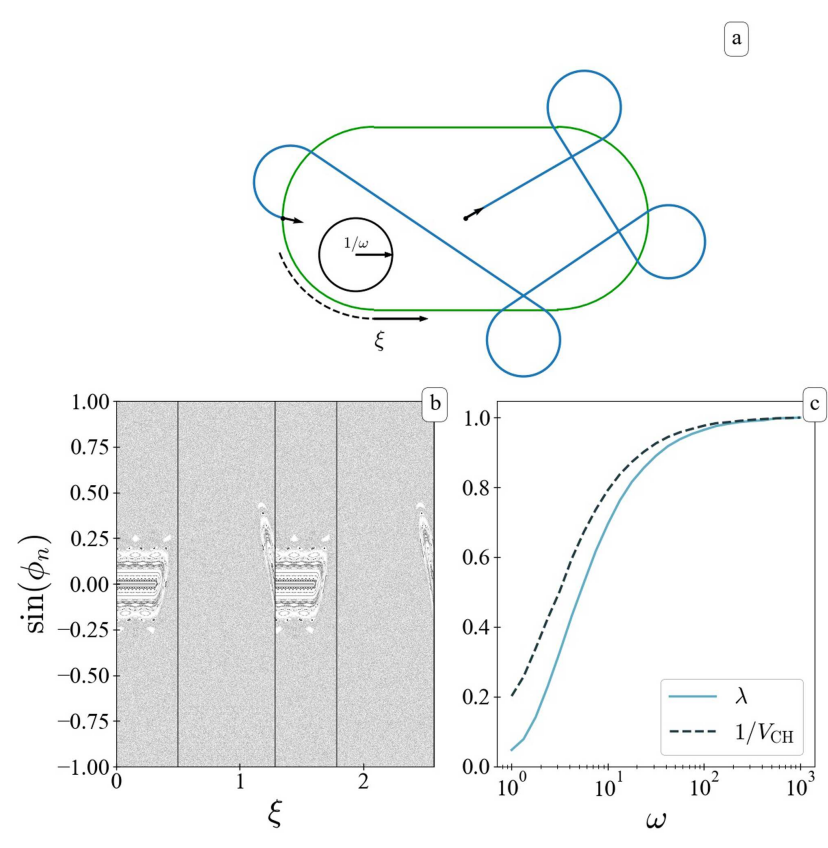

FIG. 7. The inverse stadium billiard (ISB). (a) An example orbit in the ISB (stadium width and length are 0.5). Outside the stadium, the particle undergoes circular motion with radius $1 / \omega$. (b) The boundary map (see Appendix B) of the ISB, computed for $\omega=10$. In the middle, one can see stability islands, which seem to have a fractal boundary. (c) Lyapunov exponent $\lambda$ and chaotic volume $V_{t}$ extCH vs $\omega$, both normalized to their maximums for comparison. $\lambda$ is obtained with a modified version of the $\mathrm{DPH}$ framework using tangent space evolution matrices derived by Vörös et al. in Ref. 30. $V_{C H}$ is the volume of the billiard flow of the chaotic orbits and is calculated by weighting the area of the ergodic region (obtained numerically) on the boundary map with its mean time to next collision. We only consider orbits that do intersect the billiard boundary of the ISB.

even more involved. Yet, in all three cases, we find what is suggested by our toy model: the Lyapunov exponent has a leading contribution that is inverse to the chaotic phase space volume.

Because generally chaos in billiards arises via dispersing and defocusing collisions with curved boundary segments, ${ }^{34}$ the Lyapunov exponent is necessarily inversely linked with the mean return time to these boundary segments. Furthermore, Kac's lemma dictates that the mean return time of the chaotic trajectories to these boundaries is directly proportional to the chaotic phase space volume. For this reason, we hypothesize that, for most chaotic billiards with mixed phase space, the Lyapunov exponent has a leading contribution inverse to the chaotic phase space volume.

This should straightforwardly carry over to higher dimensions as well, since Kac's lemma, the DPH framework, as well as our toy model, does not depend in any way on the dimensionality of the billiard. So far, we did not find significant differences between billiards with sharply divided phase space (like the MB and the MPSB) and a fractal phase space structure (like the ISB). To conclude whether there are fundamental differences between sharply-divided and fractal phase spaces, one will have to do more research. What we want to point out is that for fractal phase spaces, it is much harder to estimate the volume of the chaotic set.

\section{APPENDIX A: EVOLUTION OF PERTURBATION VECTOR IN A MAGNETIC FIELD}

In their paper, Dellago, Posch, and Hoover give two main equations to compute the evolution of perturbations in the tangent space along a piecewise smooth flow defined by the autonomous ODE system,

$$
\dot{\Gamma}=\mathbf{F}(\boldsymbol{\Gamma}) .
$$

During smooth propagation, the perturbation vector $\delta \boldsymbol{\Gamma}$ along the trajectory $\boldsymbol{\Gamma}$ evolves according to

$$
\delta \dot{\boldsymbol{\Gamma}}=\left.\frac{\partial \mathbf{F}}{\partial \boldsymbol{\Gamma}}\right|_{\Gamma} \cdot \delta \boldsymbol{\Gamma} .
$$

If smooth propagation is interrupted at discrete times $t_{j}(\boldsymbol{\Gamma})$ by a discontinuous jump, represented here by a differentiable $\operatorname{map} \mathbf{M}_{j}(\boldsymbol{\Gamma})$, the perturbation vector after the jump is given by

$$
\delta \boldsymbol{\Gamma}^{\prime}=\left.\frac{\partial \mathbf{M}_{j}}{\partial \boldsymbol{\Gamma}}\right|_{\boldsymbol{\Gamma}} \cdot \delta \boldsymbol{\Gamma}_{i}+\left(\left.\frac{\partial \mathbf{M}_{j}}{\partial \boldsymbol{\Gamma}}\right|_{\boldsymbol{\Gamma}} \cdot \mathbf{F}\left(\boldsymbol{\Gamma}_{i}\right)-\mathbf{F}(\mathbf{M}(\boldsymbol{\Gamma}))\right) \delta \tau_{c},
$$

where $\delta \tau_{c}=t_{j}(\boldsymbol{\Gamma}+\delta \boldsymbol{\Gamma})-t_{j}(\boldsymbol{\Gamma})$. For the case of elastic reflection with an obstacle, $\mathbf{M}_{j}$ can be written as

$$
\boldsymbol{\Gamma}^{\prime}=\mathbf{M}_{j}(\boldsymbol{\Gamma})=\left(\begin{array}{c|c}
\mathbb{I}_{2 \times 2} & 0_{2 \times 2} \\
\hline 0_{2 \times 2} & \mathbb{I}_{2 \times 2}-2(\mathbf{n} \otimes \mathbf{n})
\end{array}\right) \cdot \boldsymbol{\Gamma} .
$$

Here, $\mathbf{n}$ is the normal vector of the obstacle at the collision point, $\mathbb{I}_{2 \times 2}$ and $0_{2 \times 2}$ are the $2 \times 2$ identity and zero matrices, respectively, and $(\mathbf{a} \otimes \mathbf{b})_{j k}=a_{j} b_{k}$ is a second-order tensor.

\section{Propagation}

In magnetic billiards, particles propagate in circular arcs. To get the simplest possible set of equations of motion describing this mode of propagation, it is useful to introduce a phase angle $\theta=\arctan \left(p_{y} / p_{x}\right)$. For uniform circular motion, this phase angle grows linearly in time as the system rotates with constant angular velocity $\omega$. This can be exploited to determine $\dot{\mathbf{p}}$ using the chain rule,

$$
\dot{\mathbf{p}}=\frac{\mathrm{d} \mathbf{p}}{\mathrm{d} t}=\frac{\mathrm{d} \theta}{\mathrm{d} t} \cdot \frac{\mathrm{d} \mathbf{p}}{\mathrm{d} \theta}=\omega \cdot \frac{\mathrm{d} \mathbf{p}}{\mathrm{d} \theta} .
$$

Using $\|\mathbf{p}\|=1$, one can easily calculate the explicit relation between $\mathbf{p}$ and $\theta$,

$$
\begin{aligned}
& p_{x}=\cos (\theta) \\
& p_{y}=\sin (\theta)
\end{aligned} \Rightarrow \begin{aligned}
& \frac{\mathrm{d} p_{x}}{\mathrm{~d} \theta}=-p_{y}, \\
& \frac{\mathrm{d} p_{y}}{\mathrm{~d} \theta}=p_{x},
\end{aligned}
$$

and combine the results of Eqs. (A5) and (A6) to receive the equations of motion

$$
\mathbf{F}(\boldsymbol{\Gamma})=\left(\begin{array}{c}
\mathbf{p} \\
\omega \cdot \mathbf{R p}
\end{array}\right) \quad \text { where } \mathbf{R}=\left(\begin{array}{cc}
0 & -1 \\
1 & 0
\end{array}\right) .
$$

Using Eq. (A2), we can now state the equations of evolution for a perturbation vector $\delta \boldsymbol{\Gamma}$ using the Jacobian $\mathbf{J}$ of $\mathbf{F}$,

$$
\delta \dot{\Gamma}=\mathbf{J} \cdot \delta \boldsymbol{\Gamma}=\left(\begin{array}{c|c}
0_{2 \times 2} & \mathbb{I}_{2 \times 2} \\
\hline 0_{2 \times 2} & -\omega \cdot \mathbf{R}
\end{array}\right) \delta \boldsymbol{\Gamma} .
$$


Using an exponential ansatz, we can compute the general solution of this system and receive a final result of

$$
\begin{aligned}
\delta \boldsymbol{\Gamma}(t) & =\mathbb{B} \cdot \delta \boldsymbol{\Gamma}\left(t_{0}\right), \\
\mathbb{B} & =\left(\begin{array}{l|cc}
\mathbb{I}_{2 \times 2} & \rho \sin (\omega t) & \rho(\cos (\omega t)-1) \\
\hline 0_{2 \times 2} & -\rho(\cos (\omega t)-1) & \rho \sin (\omega t) \\
\cos (\omega t) & -\sin (\omega t) \\
\sin (\omega t) & \cos (\omega t)
\end{array}\right),
\end{aligned}
$$

where $\rho=1 / \omega$ is the cyclotron radius.

\section{Collisions}

The derivation of the collision map for $\delta \boldsymbol{\Gamma}$ is largely analogous to the process used by DPH to derive their result for nonmagnetic billiards. The equations of motion are assumed as stated in Eq. (A7). For Eq. (A3), we require the Jacobian matrix of $\mathbf{M}_{j}$, which is

$$
\frac{\partial \mathbf{M}_{j}}{\partial \boldsymbol{\Gamma}}=\left(\begin{array}{cc}
\mathbb{I}_{2 \times 2} & 0 \\
\mathbf{A} & \mathbf{B}
\end{array}\right),
$$

where

$$
\begin{aligned}
& \mathbf{A}=2\left((\mathbf{n} \otimes \mathbf{p})+\langle\mathbf{p}, \mathbf{n}\rangle \Delta \mathbb{I}_{2 \times 2}\right) \frac{\partial \mathbf{n}}{\partial \mathbf{q}}, \\
& \mathbf{B}=\mathbb{I}_{2 \times 2}-2(\mathbf{n} \otimes \mathbf{n}) .
\end{aligned}
$$

By inserting Eqs. (A7), (A4), and (A10) into (A3), we find

$$
\begin{aligned}
\delta \boldsymbol{\Gamma}^{\prime} & =\left(\begin{array}{cc}
\mathbb{I}_{2 \times 2} & 0 \\
\mathbf{A} & \mathbf{B}
\end{array}\right) \delta \boldsymbol{\Gamma}+\left[\left(\begin{array}{cc}
\mathbb{I}_{2 \times 2} & 0 \\
\mathbf{A} & \mathbf{B}
\end{array}\right)\right. \\
& \left.\cdot\left(\begin{array}{c}
\mathbf{p} \\
\omega \cdot \mathbf{R} \mathbf{p}
\end{array}\right)-\left(\begin{array}{c}
\mathbf{p}-2(\mathbf{n} \otimes \mathbf{n}) \mathbf{p} \\
\omega \cdot \mathbf{R} \mathbf{B} \mathbf{p}
\end{array}\right)\right] \delta \tau_{c} .
\end{aligned}
$$

It is now helpful to continue calculations for $\delta \mathbf{q}^{\prime}$ and $\delta \mathbf{p}^{\prime}$ separately. For the position component $\delta \mathbf{q}^{\prime}$, equation (A11) can be written as

$$
\begin{aligned}
\delta \mathbf{q}^{\prime} & =\delta \mathbf{q}+[\mathbf{p}-\mathbf{p}+2(\mathbf{n} \otimes \mathbf{n}) \mathbf{p}] \delta \tau_{c} \\
& =\delta \mathbf{q}+2 \delta \tau_{c}(\mathbf{n} \otimes \mathbf{n}) \mathbf{p} .
\end{aligned}
$$

For the momentum component $\delta \mathbf{p}^{\prime}$, we get

$$
\delta \mathbf{p}^{\prime}=\mathbf{A} \delta \mathbf{q}+\mathbf{B} \delta \mathbf{p}+\delta \tau_{c}[\mathbf{p} \mathbf{A}+\omega \mathbb{S}]
$$

where

$$
\mathbb{S}:=\mathbf{B R}-\mathbf{R B}=2\left(\begin{array}{cc}
-2 n_{1} n_{2} & n_{1}^{2}-n_{2}^{2} \\
n_{1}^{2}-n_{2}^{2} & 2 n_{1} n_{2}
\end{array}\right) .
$$

This can be simplified by using the fact that $(\mathbf{b} \otimes \mathbf{a}) \mathbf{c}=\langle\mathbf{c}, \mathbf{a}\rangle \mathbf{b}$ and introducing the quantity $\delta \mathbf{q}_{c}=\delta \mathbf{q}+\delta \tau_{c} \mathbf{p}$, which represents the real space difference vector between the collision points of satellite and reference trajectories,

$$
\begin{aligned}
\delta \mathbf{p}^{\prime}= & \delta \mathbf{p}-2\langle\delta \mathbf{p}, \mathbf{n}\rangle \mathbf{n}-2 \frac{\partial \mathbf{n}}{\partial \mathbf{q}}\left(\left\langle\mathbf{p}, \delta \mathbf{q}_{c}\right\rangle \mathbf{n}\right. \\
& \left.+\langle\mathbf{p}, \mathbf{n}\rangle \delta \mathbf{q}_{c}\right)+\delta \tau_{c} \omega \mathbb{S} \mathbf{p} .
\end{aligned}
$$

Using geometric considerations outlined by $\mathrm{DPH}$, we can now rewrite the penultimate term to get

$$
\delta \mathbf{p}^{\prime}=\delta \mathbf{p}-2\langle\delta \mathbf{p}, \mathbf{n}\rangle \mathbf{n}-2 \gamma_{R} \frac{\langle\delta \mathbf{q}, \mathbf{e}\rangle}{\cos \phi} \mathbf{e}^{\prime}+\delta \tau_{c} \omega \mathbb{S} \mathbf{p},
$$
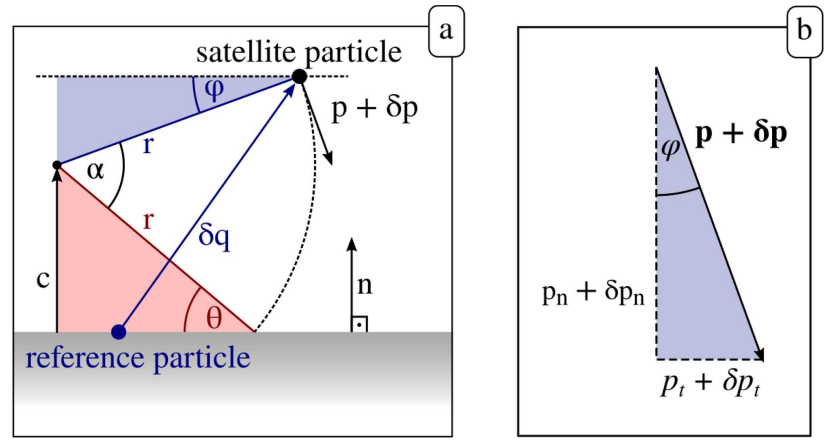

FIG. 8. (a) Geometric derivation of $\delta \tau_{c}$. As all spatial perturbations are small, it is sufficient to approximate the obstacle as a straight line. In this example, the satellite particle collides after the reference particle. (b) Decomposition of momentum into normal and tangential components.

where $\phi$ is the angle of incidence, $\gamma_{R}$ is the local curvature of the obstacle, and $\mathbf{e}$ and $\mathbf{e}^{\prime}$ are unit vectors orthogonal to $\mathbf{p}$ and $\mathbf{p}^{\prime}$, respectively.

\section{Collision delay time}

The quantity $\delta \tau_{c}$ in Eqs. (A3) and (A11) can be interpreted as the time delay in between the collisions of the reference trajectory $\boldsymbol{\Gamma}$ and its satellite $\boldsymbol{\Gamma}+\delta \boldsymbol{\Gamma}$.

It can be computed by determining the signed distance from the satellite to its collision point measured along its trajectory at the time $t_{j}(\boldsymbol{\Gamma})$ of the collision of the reference particle. As we are considering the linearized dynamics of the perturbation and Eq. (A3) is valid only to the first order of $\delta \boldsymbol{\Gamma}$, we will ignore all higher orders of $\delta \boldsymbol{\Gamma}$ in the subsequent calculations.

Furthermore, we will denote vector components in the normal direction of the obstacle by a subscript $n$, i.e., $a_{n}=\langle\mathbf{a}, \mathbf{n}\rangle$. Similarly, the tangent component of a vector will be denoted by a subscript $t$.

Geometrically, one can immediately derive the following two relations from the two triangles highlighted in Fig. 8(a):

$$
\begin{gathered}
\rho \sin \phi=\langle\delta \mathbf{q}, \mathbf{n}\rangle-\langle\mathbf{c}, \mathbf{n}\rangle \quad \text { (blue triangle) } \\
\rho \sin \theta=\langle\mathbf{c}, \mathbf{n}\rangle \quad \text { (red triangle), }
\end{gathered}
$$

where $\mathbf{c}$ is a vector between the obstacle and the cyclotron centre, as shown in Fig. 8(a). Eliminating the factor $\langle\mathbf{c}, \mathbf{n}\rangle$ yields

$$
\sin \theta=\frac{\langle\delta \mathbf{q}, \mathbf{n}\rangle}{\rho}-\sin \phi .
$$

By construction, $\theta$ cannot exceed $\frac{\pi}{2}$ in the absolute value. Therefore, we can safely apply the arcsine function to Eq. (A18), receiving an expression for $\theta$,

$$
\theta=\arcsin \left(\frac{\langle\delta \mathbf{q}, \mathbf{n}\rangle}{r}-\sin \phi\right)
$$

We can now use this to compute the angle $\alpha$ corresponding to the arc the particle has to travel during $\delta \tau_{c}$. 
Using Fig. 8(a), we can determine an expression for $\alpha$

$$
\begin{aligned}
& \alpha=\phi+\theta \\
& \stackrel{\mathrm{Eq} \cdot(\mathrm{A} 19)}{=} \phi+\arcsin \left(\frac{\langle\delta \mathbf{q}, \mathbf{n}\rangle}{r}-\sin \phi\right) .
\end{aligned}
$$

This can be further simplified by expressing $\phi$ in terms of the satellite particle's momentum [compare Fig. 8(b)],

$$
\begin{aligned}
\alpha= & \underbrace{\arcsin \left(\frac{p_{t}+\delta p_{t}}{\|\mathbf{p}+\delta \mathbf{p}\|}\right)}_{K} \\
& +\underbrace{\arcsin \left(\frac{\langle\delta \mathbf{q}, \mathbf{n}\rangle}{r}-\frac{p_{t}+\delta p_{t}}{\|\mathbf{p}+\delta \mathbf{p}\|}\right)}_{L} .
\end{aligned}
$$

We can now linearize Eq. (A21) to receive our final result for $\alpha$. As the individual terms are somewhat complicated, but very similar, we will treat them separately. The first-order Taylor expansion of leftmost term is

$$
K \approx \arcsin \left(\frac{p_{t}}{\|\mathbf{p}\|}\right)+\zeta\left(\delta \mathbf{p}_{\mathbf{t}}\right)
$$

where $\zeta$ is of the form $a \cdot \delta p_{t}+b \cdot \delta p_{n}$ with $a, b$ given by the respective partial derivatives. Expanding the rightmost term in (A21) yields the similar result of

$$
L \approx \arcsin \left(-\frac{p_{t}}{\|\mathbf{p}\|}\right)-\zeta\left(\delta \mathbf{p}_{\mathbf{t}}\right)+\frac{1}{\sqrt{1-p_{t}^{2}}} \cdot \frac{\left\langle\delta \mathbf{q}_{\mathbf{i}}, \mathbf{n}\right\rangle}{r} .
$$

This simplifies the final result for $\alpha$ significantly. Using the antisymmetry of the arcsine function, we can see that most of (A22) and (A23) cancel out, leaving only

$$
\alpha=\frac{1}{\sqrt{1-p_{t}^{2}}} \cdot \frac{\langle\delta \mathbf{q}, \mathbf{n}\rangle}{r} .
$$

Finally, we have to multiply Eq. (A24) with the cyclotron radius $r$, then divide the resulting arclength by $\|p\|$ to get $\delta \tau_{c}$.

As $\|\mathbf{p}\|=1$ per convention, we can substitute $\sqrt{1-p_{t}^{2}}=\left|p_{n}\right|$. However, we know that $p_{t}<0$ as the reference particle must have been moving toward the obstacle to collide with it. Therefore, we can further simplify our result to obtain

$$
\delta \tau_{c}=-\frac{\langle\delta \mathbf{q}, \mathbf{n}\rangle}{\langle\mathbf{p}, \mathbf{n}\rangle} .
$$

This is the same result as for linear propagation in Ref. 24 [Eq. (18)], since higher orders of $\delta \boldsymbol{\Gamma}$ were neglected.

Combining Eqs. (A12), (A15), and (A25), we receive the final result

$$
\begin{aligned}
\delta \boldsymbol{\Gamma}^{\prime}= & \left(\begin{array}{c}
\delta \mathbf{q}-2(\delta \mathbf{q} \cdot \mathbf{n}) \mathbf{n} \\
\delta \mathbf{p}-2(\delta \mathbf{p} \cdot \mathbf{n}) \mathbf{n}-2 \gamma_{r} \frac{\langle\delta \mathbf{q}, \mathbf{e}\rangle}{\cos \phi} \mathbf{e}
\end{array}\right) \\
& -\omega \frac{(\delta \mathbf{q} \cdot \mathbf{n})}{(\mathbf{p} \cdot \mathbf{n})}\left(\begin{array}{c}
0 \\
\mathbb{S} \cdot \mathbf{p}
\end{array}\right) .
\end{aligned}
$$

\section{APPENDIX B: MEAN RETURN TIME TO STEM}

In this section, we will derive an analytic expression for the mean return time to the stem bottom in a mushroom billiard. Using Kac's lemma, ${ }^{31-33}$ which states that for volume-preserving maps, the mean number of iterations $n_{S}$ required to return to a compact subset $S$ of phase space is given by

$$
n_{S}=\frac{\mu(A)}{\mu(S)},
$$

where $\mu(\cdot)$ is the volume of a set and $A$ is the subset of phase space accessible to orbits originating in $S$.

To transform the billiard flow into a map, we discretize time in small steps $\Delta t$, implicitly considering the limit of $\Delta t \rightarrow 0$. We now choose the set $S$ of momenta and positions defined by

$$
S=\left\{\mathbf{q} \in Y, p_{y}>0\right\},
$$

where $Y$ is a box of width $w$ and height $\epsilon$ at the bottom of the stem. One should be careful about the choice of $S$. The simplistic approach of choosing the cap semicircle as the returning set (since the elementary growth segments are delimited by collisions with curved boundaries) will not yield the correct result. That is because the mean return time to the cap semicircle inherently includes contributions from both the periodic orbits of the MB and the laminar episodes of the elementary growth segments, which we have already shown to correspond to "free flight"-like motion.

The phase space volume of $S$ is $\mu(S)=\pi w \epsilon$ in the limit of $\Delta t \rightarrow 0$. As the chaotic phase space component of mushroom billiards is ergodic, ${ }^{19}$ we know that the measure of the subset $A$ of phase space accessible from $S$ is given by the volume of chaotic phase space $V_{\mathrm{CH}}=V_{\mathrm{TOT}}-V_{\mathrm{REG}}$.

Applying Kac's lemma to get the mean iterations to return to the stem and multiplying by $\Delta t$, we get a result for the mean return time $\kappa_{S}(h, w, r)$ to the set $S$,

$$
\kappa_{S}(h, w)=\frac{V_{\mathrm{CH}}(h, w)}{\pi \epsilon w} \Delta t .
$$

To eliminate $\Delta t$, we divide by $\kappa_{S}$ for $w=2$ to get

$$
\kappa_{S}(h, w)=\frac{V_{\mathrm{CH}}(h, w)}{V_{\mathrm{TOT}}(h, 2)} \frac{2}{w} \kappa_{S}(h, 2) .
$$

As this equation no longer depends on $\epsilon$, we can now take the limit $\epsilon \rightarrow 0$. The reason to use $w=2$ here is because the MB becomes the stadium billiard for $w=2$ (and thus is fully chaotic with no regular components with measure $>0$ ).

We can find $\kappa_{S}(h, 2)$ because of the ergodicity of the MB for $w=2$. Specifically, it holds that $\kappa_{S}(h, 2)=n_{S}(h) \times \tau$ with $n_{S}$ being the mean amount of iterations to return to the stem and $\tau$ being the mean collision time in the MB given by Eq. (3). We consider the boundary map of the billiard (Birkhoff coordinates), $\left(\xi, \sin \phi_{n}\right)$, with $\xi$ being the coordinate along the boundary (i.e., the arc-length) and $\phi_{n}$ being the angle of incidence with respect to the normal vector at $\xi$. This coordinate system is a discrete mapping and Kac's lemma applies directly. Therefore, the mean iterations to return to the stem 
bottom are

$$
n_{S}=\frac{2|\partial Q|}{2 \cdot 2},
$$

where $|\partial Q|$ is the perimeter the boundary (the explicit factor of 2 represents the contribution of $\sin \phi_{n}$ ). Using Eq. (3), we find

$$
\tau=\frac{\pi \cdot(2 h+\pi / 2)}{|\partial Q|} .
$$

Combining Eqs. (B5) and (B6), we receive an expression for the mean stem return time in the fully ergodic case

$$
\kappa_{S}(h, 2)=\frac{\pi}{2}(2 h+\pi / 2) .
$$

This expression can be simplified by substituting the total phase space volume as defined in Eq. (25), yielding

$$
\kappa_{S}(h, 2)=\frac{V_{\text {TОT }}(h, 2)}{4} .
$$

Inserting this result into Eq. (B4), we receive

$$
\kappa_{\mathrm{MB}}(h, w)=\frac{V_{\mathrm{CH}}(h, w)}{2 w} .
$$

\section{APPENDIX C: MEAN DURATION OF CHAOTIC EPISODES}

A chaotic episode in the MB as defined above consists of the particle travelling from the cap head directly into the stem and back up to the cap head, without any other collisions inside the cap. To determine the mean duration $\tau_{c}$ of these episodes, we can geometrically determine the average lengths of the trajectories, exploiting that all trajectories are uniquely defined by the angle of incidence $\alpha$ and the distance $\delta$ from the cap center at which they enter the stem.

To simplify the calculation, it is useful to split the trajectory into the mean length of cap transit $\langle c\rangle$ and the mean length of stem transit $\langle s\rangle$, where $\tau_{c}=2\langle c\rangle+2\langle s\rangle$.

The stem transit length can be easily computed using simple trigonometry and depends only on the angle $\alpha$

$$
s=\frac{h}{\cos (\alpha)} .
$$

As the directions of particle momenta are equidistributed, we know that $\alpha$ must be cosine-distributed. We can now integrate over $\alpha$ to obtain the mean $s$, finding

$$
\langle s\rangle=\int \frac{h}{\cos \alpha} \frac{1}{2} \cos (\alpha) d \alpha=\pi h .
$$

Determining the cap transit length is more difficult as it depends on both $\alpha$ and $\delta$. Using the law of sines, we can derive

$$
c=\frac{r}{\cos (\alpha)} \cos \left(\operatorname{asin}\left(\frac{\delta}{r} \cos \alpha\right)-\alpha\right) .
$$

To get an average result, this expression has to be integrated both over $\delta$ and $\alpha$, again using the fact that $\alpha$ is cosine-distributed. Unfortunately, we were unable to solve the integrals analytically. Therefore, we decided to approximate $\int c d \delta$ by a polynomial before performing the second integration, yielding

$$
\langle c\rangle \approx 1-\frac{w^{2}}{36}-\frac{w^{4}}{1200}-\cdots .
$$

\section{APPENDIX D: PROOF OF EQ. (1)}

We are interested in the flow of the PSB but to apply Kac's lemma, we need a discrete system. Thus, we obtain a map of the flow $\Phi^{t}$ by discretizing in time (similarly with Appendix B), $f:=\Phi^{\Delta t_{\varepsilon}}$, with $\Delta t_{\varepsilon} \sim \varepsilon$. To prove Eq. (1), we will apply Kac's lemma to a set $\mathcal{S}$.

Let $\mathcal{T}_{\mathcal{S}}(B)=\Delta t_{\varepsilon} \times n_{\mathcal{S}}(B)$ (where $B$ is the magnetic field) denote physical recurrence time instead of map iterations. Let $\mathcal{W}$ be a circle of radius $r+\varepsilon$ concentric to the disc of the PSB and define the phase space subset $\mathcal{S}_{\varepsilon}$ such that

$$
\mathcal{S}_{\varepsilon}=\{\mathbf{x}, \mathbf{v}: \mathbf{x} \in \mathcal{W} \text { and } \mathbf{v} \cdot \eta(\mathbf{x})<0\},
$$

where $\eta(\mathbf{x})$ is the vector normal to $\mathcal{W}$. The mean collision time $\kappa$ of the PBS is exactly $\mathcal{T}_{\mathcal{S}}$ in the limit $\varepsilon \rightarrow 0$.

To find $\mu(\mathcal{S} ; B)$, the measure of set $\mathcal{S}$, we first realize that it does not depend on the magnetic field, $\mu(\mathcal{S} ; B)=\mu(\mathcal{S} ; 0)=\mu(\mathcal{S})$. This is due to the infinitesimal width of $\mathcal{S}$, over which motion can always be approximated by a straight line for all finite magnetic fields values (i.e., equalling the magnetic field free case). Then, using (B1) at $B=0$, we have

$$
\mathcal{T}_{\mathcal{S}}(B=0)=\left(\frac{\Delta t_{\varepsilon}}{\mu\left(\mathcal{S}_{\varepsilon}\right)}\right) \mu(A ; B=0)=\left(\frac{\Delta t_{\varepsilon}}{\mu\left(\mathcal{S}_{\varepsilon}\right)}\right),
$$

because the PSB without magnetic field is fully ergodic and thus $\mu(A ; B=0)=\mu(M)=1$ (here $M$ denotes the entire phase space whose measure we set for simplicity to 1$)$. By substitution, we get $\mathcal{T}_{\mathcal{S}}(B)=\mu(A ; B) \times \mathcal{T}_{\mathcal{S}}(0)$. For small enough magnetic fields, all chaotic orbits (and up to measure 0 only those) collide with the discs. In the limit $\varepsilon \rightarrow 0$, both $\Delta t_{\varepsilon}$ and $\mu\left(\mathcal{S}_{\varepsilon}\right)$ go to 0 linearly with $\varepsilon$; therefore, their ratio converges, i.e., $\mathcal{T}_{\mathcal{S}} \rightarrow \kappa$. Since by definition $\mu(A)=g_{c}$, the portion of chaotic orbits in the PSB (because we set the entire volume to have measure 1), we find that the mean collision time is given by the fraction of chaotic orbits as a function of the magnetic field $B$, times the mean collision time at $B=0$

$$
\kappa(B)=g_{c}(B) \times \kappa(0) .
$$

\section{REFERENCES}

${ }^{1}$ Y. G. Sinai, Russ. Math. Surv. 25, 137 (1970).

${ }^{2}$ L. A. Bunimovich, Funct. Anal. Appl. 8, 254 (1974).

${ }^{3}$ L. A. Bunimovich, Commun. Math. Phys. 65, 295 (1979).

${ }^{4}$ H.-J. Stöckmann and J. Stein, Phys. Rev. Lett. 64, 2215 (1990).

${ }^{5}$ A. H. Barnett and T. Betcke, Chaos 17, 043125 (2007).

${ }^{6}$ A. D. Stone, Nature 465, 696 (2010).

${ }^{7}$ C. Gmachl, F. Capasso, E. E. Narimanov, J. U. Nöckel, A. D. Stone, J. Faist, D. L. Sivco, and A. Y. Cho, Science 280, 1556 (1998).

${ }^{8}$ S. Koyanagi, T. Nakano, and T. Kawabe, J. Acoust. Soc. Am. 124, 719 (2008)

${ }^{9}$ G. Datseris, T. Geisel, and R. Fleischmann, New J. Phys. 21, 043051 (2019).

${ }^{10}$ R. Fleischmann, T. Geisel, and R. Ketzmerick, Phys. Rev. Lett. 68, 1367 (1992).

${ }^{11}$ D. Weiss, M. L. Roukes, A. Menschig, P. Grambow, K. von Klitzing, and G. Weimann, Phys. Rev. Lett. 66, 2790 (1991). 
${ }^{12}$ N. I. Chernov, G. L. Eyink, J. L. Lebowitz, and Y. G. Sinai, Commun. Math. Phys. 154, 569 (1993).

${ }^{13}$ R. Yagi, R. Sakakibara, R. Ebisuoka, J. Onishi, K. Watanabe, T. Taniguchi, and Y. Iye, Phys. Rev. B. 92, 195406 (2015).

${ }^{14}$ H. Maier, J. Ziegler, R. Fischer, D. Kozlov, Z. D. Kvon, N. Mikhailov, S. A. Dvoretsky, and D. Weiss, Nat. Commun. 8, 1 (2017); e-print arXiv:1708.07766.

${ }^{15}$ S. Chen, Z. Han, M. M. Elahi, K. M. M. Habib, L. Wang, B. Wen, Y. Gao, T. Taniguchi, K. Watanabe, J. Hone, A. W. Ghosh, and C. R. Dean, Science 353, 1522 (2016). arXiv:1602.08182.

${ }^{16}$ Electron Transport in Quantum Dots, edited by J. P. Bird (Springer, New York, 2003).

${ }^{17}$ C. Beenakker, in Quantum Dots: A Doorway to Nanoscale Physics (Springer, Berlin, Heidelberg, 2005), pp. 131-174.

${ }^{18}$ L. A. Ponomarenko, F. Schedin, M. I. Katsnelson, R. Yang, E. W. Hill, K. S. Novoselov, and A. K. Geim, Science 320, 356 (2008).

${ }^{19}$ L. A. Bunimovich, Chaos 11, 802 (2001)

${ }^{20} \mathrm{~N}$. Chernov, J. Stat. Phys. 88, 1 (1997).

${ }^{21} \mathrm{~N}$. Chernov and L. S. Young, in Hard Ball Systems and the Lorentz Gas, Encyclopedia of Mathematical Sciences (Springer, Berlin, Heidelberg, 2000), pp. 89-120.
${ }^{22}$ N. Chernov and R. Markarian, Chaotic Billiards, mathematic ed. (American Mathematical Society, 2006), p. 328.

${ }^{23}$ C. Dellago and H. A. Posch, Phys. Rev. E 52, 2401 (1995).

${ }^{24}$ C. Dellago, H. A. Posch, and W. G. Hoover, Phys. Rev. E 53, 1485 (1996).

${ }^{25}$ N. I. Chernov, Funct. Anal. Appl. 25, 204 (1991).

${ }^{26}$ P. L. Garrido, J. Stat. Phys. 88, 807 (1997).

${ }^{27}$ P. Gaspard and F. Baras, Phys. Rev. E 51, 5332 (1995).

${ }^{28} \mathrm{H}$. A. Posch and R. Hirschl, in Hard Ball Systems and the Lorentz Gas, Encyclopedia of Mathematical Sciences (Springer, Berlin, Heidelberg, 2000), pp. 279-314.

${ }^{29}$ O. Meplan, F. Brut, and C. Gignoux, J. Phys. Math. Gen. 26, 237 (1993).

${ }^{30}$ Z. Vörös, T. Tasnádi, J. Cserti, and P. Pollner, Phys. Rev. E 67, 065202(R) (2003)

${ }^{31}$ M. Kac, Bull. Am. Math. Soc. 53, 1002 (1947).

${ }^{32}$ R. S. MacKay, J. Nonlinear Sci. 4, 329 (1994).

${ }^{33}$ J. D. Meiss, Chaos 7, 139 (1997).

${ }^{34}$ L. A. Bunimovich, Nonlinearity 31, R78 (2018).

${ }^{35}$ G. Datseris, J. Open Source Softw. 2, 458 (2017).

${ }^{36}$ E. G. Altmann, A. E. Motter, and H. Kantz, Chaos 15, 033105 (2005).

${ }^{37}$ E. G. Altmann, A. E. Motter, and H. Kantz, Phys. Rev. E 73, 026207 (2006). 


\section{$63^{\text {rd }}$ paper: Phase space analysis of quantum transport in graphene}

At the date of submission of this thesis this paper is submitted for peer-review. Its preprint is stated as G. Datseris and R. Fleischmann, Phase space analysis of quantum transport in graphene, arXiv:1905.06637.

Author contributions GD and RF conceptualized the project. GD wrote the computer code for the Husimi function (quantum transport simulations were performed with Kwant [29]), did the simulations and data analysis. GD and RF defined measures for integrated transmission and intervalley scattering. GD prepared the figures and a first version of the manuscript. Both authors discussed the results and reviewed and edited the manuscript. 


\title{
Phase space analysis of quantum transport in graphene
}

\author{
George Datseris* and Ragnar Fleischmann \\ Max Planck Institute for Dynamics and Self-Organization and \\ Institute for the Dynamics of Complex Systems, Georg-August-Universität Göttingen
}

(Dated: Friday $17^{\text {th }}$ May, 2019)

\begin{abstract}
Prominent among the many fascinating properties of graphene are its surprising electronic transport characteristics which are commonly studied theoretically and numerically within the LandauerBüttiker formalism. Here a device is characterized by its scattering properties to and from reservoirs connected by perfect semi-infinite leads, and transport quantities are derived from the scattering matrix. In many respects, however, the device becomes a "black box" as one only analyses what goes in and out. Here we use the Husimi function as a complementary tool for understanding transport in graphene nanodevices. It is a phase space representation of the scattering wavefunctions that allows to link the scattering matrix to a more semiclassical and intuitive description and gain additional insight in to the transport process. In this article we demonstrate the benefits of the Husimi approach by analysing Klein tunneling and intervalley scattering in two simple graphene nanostructures.
\end{abstract}

\section{INTRODUCTION}

Graphene is a fascinating material for studying quantum transport, due to the abundance of new physics it brought into light quite quickly after its discovery. The possibility to measure the quantum Hall effect at room temperature $^{1}$, weak (anti-)localization effects connected with the existence of two inequivalent valleys ${ }^{2}$, the Klein tunnel effect and its potential impact on technological applications $^{3}$ or the so called universal minimum ballistic conductivity ${ }^{4}$ are just a few examples of its intriguing electronic properties (for a review of electronic transport in graphene we point to ${ }^{5}$. Most of the exciting new physics of graphene stems from the fact that the carriers for small doping follow hyper-relativistic (Dirac) dynamics.

Most quantum transport simulations of graphene nanostructures are based on the Landauer-Büttiker formalism. Its main ingredient is the scattering matrix of the device which can be calculated using techniques like the (non-equilibrium) recursive Green's functions method in tight binding models. The scattering matrix approach gives a wealth of information on transport through the device, even though in the end in some respects the device appears to be a black box. Most transport quantities, for example conductivity, thermal conductivity and even tunneling of individual modes are straightforward to obtain. For the case of zigzag graphene nanoribbons (which we present in detail in sec. IB) it is even possible to extract information about intervalley scattering (for a definition please see sec. II B), since all modes are valley polarized.

A weakness of the scattering matrix approach only becomes apparent when one wants to connect the quantitative results it produces to the physical intuition and if one wants to understand the role played by the different

* george.datseris@ds.mpg.de components of a complex (not easy to compartmentalize) device. In that case one wants to analyse the scattering wavefunctions inside the device, and how they populate position and momentum space. For example, besides the valley polarization of incoming and outgoing waves, it can be reasonable to wonder about the valley occupation inside complicated devices, and specifically in the different parts that constitute the devices.

In order to complement the scattering matrix information, and to get an intuitive connection with the semiclassical picture, here we will use the Husimi function $Q$ which transforms a wavefunction into a phase space (quasi-)distribution.

Husimi functions, that in many respects give a more convenient phase space representation of quantum states than the Wigner function, have been introduced to quantum mechanics a long time ago ${ }^{6}$. They have been used in various areas of physics. For example in such different fields as quantum optics, where they are a standard tool $^{7,8}$, and ocean acoustics ${ }^{9}$. Their most prominent role Husimi functions probably play in the field of quantum chaos which tries to unravel the properties of complex quantum systems. They have for example been used to understand the structure of the eigenfunctions in paradigmatic chaotic systems like quantum maps and billiards ${ }^{10-14}$, transport in quantum ratchets ${ }^{15}$, the dynamics of Bose-Einstein-Condensates in double well potentials $^{16}$ and the properties of optical microdisc lasers ${ }^{17-19}$. While Husimi functions have e.g. also been used to study electronic transport in disordered systems ${ }^{20}$, in general solid state physics does not yet take much advantage of this very useful tool.

Recently, in tight-binding models of nanodevices Mason et al. ${ }^{21-23}$ have introduced a processed Husimi map allowing to recover and visualize classical paths in coordinate space. In particular they used the Husmimi map to study graphene billiard systems ${ }^{21}$. We will show below that the direct application of the Husimi function as a distribution in position and momentum can as well be a powerful tool to understand transport in graphene 
nanodevices, because, for example, it offers readily accessible information on the actual angle of incidence at barriers within the device, or it reveals where in momentum space the scattering wavefunction gets localized at different spatial positions in the nanodevice.

In this article we will study Klein tunneling and intervalley scattering in tight-binding models of simple, exemplary graphene nanodevices. We use the recursive Green's function method to obtain the scattering matrices and the scattering wavefunctions in the devices, using the software Kwant $^{30}$. We then analyze the wavefunctions using the Husimi projection and show, by comparing to the scattering matrix results, that the combination of Husimi functions and semiclassical considerations allows us to interpret and understand the observed transport phenomena. The observations we report include among others the mode dependence of intervalley scattering at a pn-junction, the quantification of intervalley scattering at a tilted graphene edge and the evolution of Klein tunneling at a pn-junction at high Fermienergies, when the dispersion relation deviates from the Dirac approximation.

\section{MODEL}

\section{A. The Husimi function}

Let $\left|\mathcal{W}\left(\mathbf{r}_{0}, \mathbf{k}_{0}, \sigma\right)\right\rangle$ denote a Gaussian wavepacket. In position representation and in the absence of magnetic fields this is simply ${ }^{24}$

$$
\mathcal{W}\left(\mathbf{r}, \mathbf{r}_{0}, \mathbf{k}_{0} ; \sigma\right)=N_{\sigma}^{D / 2} \exp \left(-\frac{\delta \mathbf{r}^{2}}{4 \sigma^{2}}+i \mathbf{k}_{0} \cdot \delta \mathbf{r}\right)
$$

(with $\delta \mathbf{r}=\mathbf{r}-\mathbf{r}_{0}$ and $D$ spatial dimensions) which is a Gaussian envelope in space with origin $\mathbf{r}_{0}$ multiplying a plane wave with wavevector $\mathbf{k}_{0} \cdot N_{\sigma}=(\sigma \sqrt{2 \pi})^{-1}$ is the normalization factor in the case of continuous space, so that $\langle W \mid W\rangle=1$ and that $\Delta x=\Delta y=\sigma$. The key property of these wavepackets is that they minimize the uncertainty relation between position and momentum. Here $\sigma$ is the spatial uncertainty and thus is a parameter that controls the trade-off between the uncertainty in position $(\sigma)$ or momentum space $(1 /(2 \sigma))$.

The Husimi function is defined as the magnitude of a projection of a wavefunction onto $|\mathcal{W}\rangle^{6,24-26}$

$$
Q\left(\mathbf{r}_{0}, \mathbf{k}_{0} ; \sigma\right)=\frac{1}{\pi}\left|\left\langle\psi \mid \mathcal{W}\left(\mathbf{r}_{0}, \mathbf{k}_{0} ; \sigma\right)\right\rangle\right|^{2}
$$

where for continuous space systems we have

$$
\left\langle\psi \mid \mathcal{W}\left(\mathbf{r}_{0}, \mathbf{k}_{0} ; \sigma\right)\right\rangle=\int \psi^{*}(\mathbf{r}) \times \mathcal{W}\left(\mathbf{r}, \mathbf{r}_{0}, \mathbf{k}_{0} ; \sigma\right) d \mathbf{r}
$$

where the integration extends over the full spatial domain of the device (in our case in two dimensions). For a tight binding system the projection is turned into a sum due to the discrete nature of the lattice

$$
\left\langle\psi \mid \mathcal{W}\left(\mathbf{r}_{0}, \mathbf{k}_{0}, \sigma\right)\right\rangle=\sum_{j} \psi^{*}\left(\mathbf{r}_{j}\right) \times e^{-\frac{\delta \mathbf{r}_{j}^{2}}{4 \sigma^{2}}} e^{i \mathbf{k}_{0} \cdot \delta \mathbf{r}_{j}}
$$

with $\delta \mathbf{r}_{j}=\mathbf{r}_{j}-\mathbf{r}_{0}, \psi\left(\mathbf{r}_{j}\right) \equiv \psi_{j}$ being the wavefunction at lattice site $j$ with position $\mathbf{r}_{j}{ }^{27}$. The normalization factors here depend on the lattice. We use $Q$ in its original form as defined by eq. (2) and we do not process it further in any way .

$Q$ is the Weierstrass transform of the Wigner function and thus is a rigorous method for transforming a wavefunction into a phase space distribution. It is versatile tool for understanding complex quantum and other wave dynamics ${ }^{9-26}$.

\section{B. Graphene devices}

We study transport in the tight binding models of the two graphene-based devices shown in Fig. 1. Device A is the conceptually simplest device in which one can study Klein tunneling in a realistic scenario (i.e. a finite nanodevice): a graphene nanoribbon (GNR) of constant width with a $\mathrm{p}-\mathrm{n}$ junction in its middle. In device $\mathrm{A}$ the boundary conditions are chosen such that it forms a "zigzag" nanoribbon. These have been studied by Bray and Fertig in detail within the Dirac approximation ${ }^{28}$ and many of their properties are known analytically (for small Fermi energies). Analytical descriptions in this case are possible because of the many symmetries that are present. Device B however breaks both, the conservation of $k_{y}$ as well as the reflection symmetry along the $x$ axis. Note also that for $\omega=\pi / 6$ the "scattering edge" in device B (highlighted in green in Fig. 1c) exactly is an armchair boundary. In both devices we create $p-n$ junctions via a linear increment of the potential energy from the $\mathrm{n}$ region with $-V_{0} / 2$ to the $\mathrm{p}$ region with $+V_{0} / 2$ over a range $w$ (see Fig. 1). The kinetic energy $E$ of the incoming electrons is connected to the Fermi energy $E_{F}$ by $E=E_{F}+V_{0} / 2$.

Zigzag GNRs have a dispersion relation shown in Fig. 1a and discussed in detail in ${ }^{28,29}$. For a given Fermi energy $M$ bands of the dispersion intersect the energy level at positive slope, thus having positive group velocity. This results in $M$ incoming ( $M$ is always odd and scales linearly with the width of the GNR). We order the modes by decreasing $k_{x}$, as shown in Fig. 1a. Importantly, for small energies the two (inequivalent) Dirac valleys $K, K^{\prime}$ are well separated in momentum space, which leads to the incoming modes being "valley-polarized". This means that modes 1 to $\lfloor M / 2\rfloor$ (where $\lfloor\cdot\rfloor$ denotes the integer part) come from valley $K^{\prime}$, while modes $\lfloor M / 2\rfloor+1$ to $M$ come from valley $K$. We also stress that $K$ has one additional incoming mode, see Fig. 1a.

All of our quantum transport simulations are tight binding calculations performed with the software 

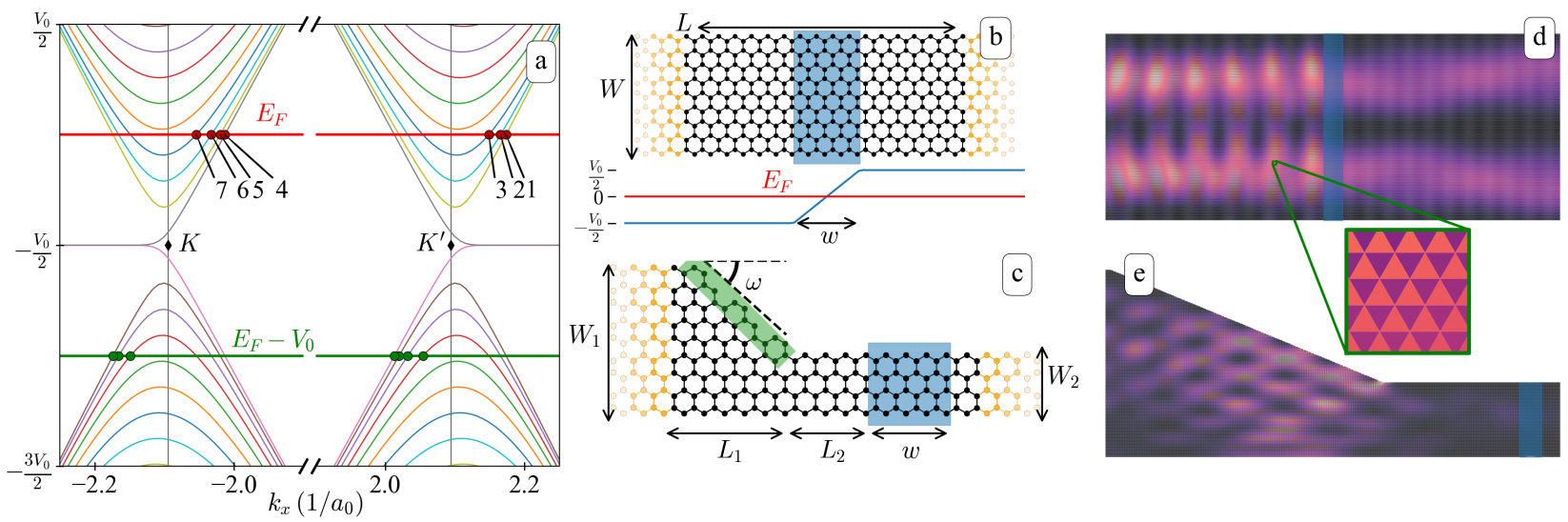

FIG. 1. Graphene-based nanodevices. (a) Dispersion relation of a zigzag graphene nanoribbon, which is separated into two inequivalent Dirac valleys for small energies. Sketched are the levels of the incoming (red) and outgoing (green) energy as well as the incoming and outgoing modes (intersections with the bands). As our transport setup is from the left to the right lead, only modes with positive group velocity (slope at the intersection) are valid. Incoming mode numbers are also shown. (b) Sketch of device A, a simple graphene nanoribbon with a p-n junction (blue). Below the device we sketch the potential profile of the p-n junction. (c) Sketch of device B. With green we highlight the scattering edge. Leads are colored orange in both sketches. (d, e) A scattering wavefunction amplitude inside simulated devices A and B respectively. The inset is showing how we plot the wavefunctions: for each sublattice we use a different marker (up or down triangles).

$\mathrm{Kwant}^{30}$. The devices are finite scattering regions that are coupled to semi-infinite leads (which are also GNR). The modes (eigenfunctions) of the leads enter the device and are subsequently scattered, giving rise to the scattering matrix $S$ and the scattering wavefunctions for each mode $\psi_{m}{ }^{31}$. From $S$ one can easily compute the transmission from one lead to another (i.e. conductance) as well as other useful quantities ${ }^{32}$. As we consider transport always from the left to the right lead, we define $\mathcal{T}$ to be the transmission matrix with $N$ rows and $M$ columns, where $M$ and $N$ are the total number of modes in the left and right lead respectively. The element $\mathcal{T}_{n m}$ is the transmission amplitude from the $m$-th (incoming) mode of the left lead to the $n$-th (outgoing) mode of the right lead. Similarly, we define $\mathcal{R}$ to be the $M \times M$ reflection matrix. In this paper we will be particularly interested in the total transmission probability $T_{m}$ of each individual incoming mode given by

$$
T_{m}=\sum_{i=1}^{N}\left|\mathcal{T}_{i m}\right|^{2}
$$

\section{Calculating the Husimi function}

For each scattering wavefunction $\psi_{m}$ we compute the Husimi function $Q$. It is of course defined on the whole phase space of the device. Not only for numerical reasons, however, but also to expose the important information, it is reasonable to calculate and analyse $Q$ only on certain well chosen subregions of phase space, and especially to reduce the dimensionality of the data we need to understand. We therefore need to specify the positions $\mathbf{r}_{0}$ and wavevectors $\mathbf{k}_{0}$ where we want to determine the distribution. For the positions we choose transverse cuts at appropriate $x_{0}$ coordinates e.g. just before and after the p-n junction in device A, i.e. we keep $x_{0}$ fixed and vary $y_{0}$. (In practice we use slices of width $3 \sigma$ around the cuts to evaluate Eq. (4).) We will thus obtain a distribution of incoming and outgoing wavevectors that "pass through" these cuts as a function of the transverse coordinate $y$.

In the following we want to reduce the dimensionality of $\mathrm{Q}$ further by exploiting energy conservation. Before we do this we need do some general consideration. The dispersion relation of an infinite graphene sheet is well known $^{33}$ (see $^{34}$ for a derivation)

$$
\begin{aligned}
\epsilon_{\lambda}(\mathbf{k}) & =\lambda t \sqrt{3+f(\mathbf{k})}-t^{\prime} f(\mathbf{k}) \\
f(\mathbf{k}) & =2 \cos \left(\sqrt{3} k_{x} a\right)+4 \cos \left(\frac{\sqrt{3}}{2} k_{x} a\right) \cos \left(\frac{3}{2} k_{y} a\right)
\end{aligned}
$$

where $a \approx 0.142 \mathrm{~nm}$ is the carbon-carbon distance (the Bravais lattice constant is $\left.a_{0}=\sqrt{3} a\right), \lambda= \pm 1$ is the band index, $t \approx 2.8 \mathrm{eV}$ is the nearest neighbor hopping and $t^{\prime}$ is the next-nearest neighbor hopping, which we consider 0 here for simplicity. We plot eq. (6) in Fig. 2a.

In nanoribbons the situations is of course different though, as the dispersion is one-dimensional (not known analytically) with multiple positive energy bands depending on the width $W$ of the ribbon (sec. IB). For each mode the incoming longitudinal wavevector $k_{x}$ (inside the lead) is known (numerically) but $k_{y}$ is not. For small energies one can use the theory of Brey \& Fertig ${ }^{28}$ which states that the transverse wavefunctions (accessible from Kwant $^{30}$ ) are pure sine modes in each sublattice. Fitting a sine wave therefore also yields the incoming $k_{y}$. Al- 

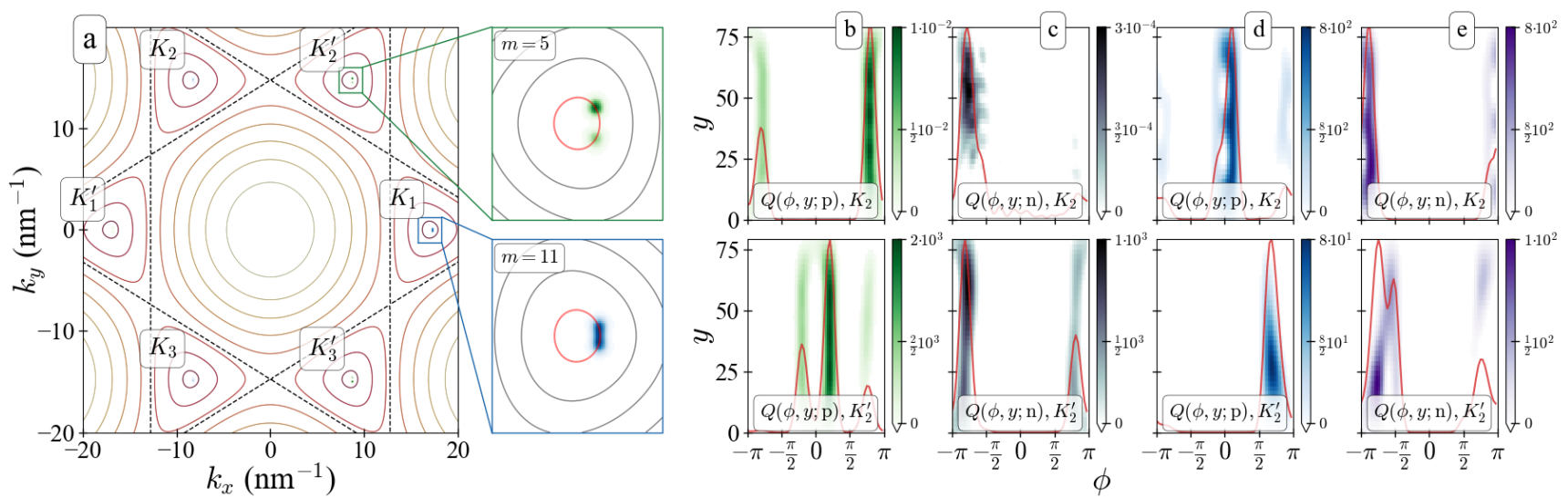

FIG. 2. Husimi functions in device A. (a) Dispersion relation of graphene (red-yellow color, dashed black line for $E=t=2.8 \mathrm{eV}$ ) and Husimi functions for 2 incoming modes (blue and green respectively) over the entire Brillouin zone, for $\mathbf{r}_{0}=\left(\frac{L}{2}, \frac{W}{2}\right), E_{F}=$ $0.2 \mathrm{eV}, V_{0}=0 \mathrm{eV}, W=80 \mathrm{~nm}$ leading to $M=17$ incoming modes. With $K_{i}^{\left({ }^{\prime}\right)}$ we label the six valleys. In the insets, the bright-red colored contour is noting the incoming energy of the simulation, here $0.2 \mathrm{eV}$. (b) Husimi distribution functions over wavevector angles $\phi$ and positions $y$ in device $\mathrm{A}, 3 \sigma \approx 24 \mathrm{~nm}$ before the p-n junction (i.e. incoming \& reflected) for valleys $K_{2}, K_{2}^{\prime}$, see sec. I C. Here $V_{0}=0.4, E_{F}=0.0 \mathrm{eV}$ (i.e. same incoming energy as panel (a)). (c) Same as (b) but $Q$ is measured $3 \sigma \approx 24 \mathrm{~nm}$ after the p-n junction (i.e. transmitted). For (b, c) the mode number is $m=5$. (d, e) Same as (b, c) but for mode number $m=11$. Over all $Q(\phi, y)$ we plot the marginal distribution $Q(\phi ; \xi)$ eq. (7) (in arbitrary units) with red color.

though this approach fails at high energies (see sec. IID), it gives a very accurate representation of the incoming wavevectors for each mode at low energies. Because device A has constant width and the potential is independent of $y$, the incoming wavevector angle is conserved up until the p-n junction, since $k_{y}$ is conserved throughout. We will use this fact in sec. II A to confirm how robust and accurate the application of the Husimi function is.

Our aim will be to use the $2 \mathrm{D}$ dispersion relation Eq. (6) to reduce the dimensionality and translate the two dimensional wave vectors into a single propagation angle. To show that this is possible with good accuracy, we first compute $Q$ over the entire Brillouin zone (BZ) at a fixed spatial position $\mathbf{r}_{\mathbf{0}}$ in the center of device $\mathrm{A}$ (i.e. at $\mathbf{r}_{0}=\left(\frac{L}{2}, \frac{W}{2}\right)$ ) in the case $V_{0}=0$ (i.e. without potential step). Fig. 2a shows $Q\left(k_{x}, k_{y}\right)$ for two different modes. We see that $Q$ localizes perfectly on top of the two-dimensional energy contour at $E_{F}$, even though the system width is only $80 \mathrm{~nm}$. This is also true for higher energies when the the Dirac approximation fails as we will show in sec. II D. $Q$ also distinguishes the inequivalent valleys excellently, since the blue and green modes of Fig. 2 are localized in different valleys (as mentioned in sec. IB we know exactly which is the incoming valley for zigzag GNR).

The above observations allows now us to reduce the dimensionality of $Q$ by using the $2 \mathrm{D}$ dispersion. What we do in the following is populate the incoming energy contour with wavevectors using equally spaced angles and measure $Q$ for these wavevectors. For energies below $t$ (dashed contour line in Fig. 2) this is done for all six valleys and the angle is measured with respect to the Dirac points, i.e. in each valley the energy contour is populated by wavevectors with equally spaced angles. Then instead of simply $Q(\ldots)$ we have $Q(\ldots ; \xi)$, where $\xi$ counts the valleys $\left(\xi \in\{1,2,3\}\right.$ means $K, \xi \in\{4,5,6\}$ means $\left.K^{\prime}\right)$. For energies above $t$ the angle is measured with respect to the center of the $\mathrm{BZ}$ and there is no $\xi$ index. This reduces our distribution $Q\left(k_{x}, k_{y}, y ; x\right)$ ( $x$ is constant) from depending on both $k_{x}, k_{y}$ to be only a function of the wavevector angle $\phi$, i.e. $Q(\phi, y ; x)$. The parameter $\sigma$ we will choose such that the wavevector uncertainty satisfies $\Delta k / k=0.2$, where $k$ is the (average) magnitude of the wavevector with respect to the center from which we measure angle. As we show in appendix A this relative wavenumber uncertainty corresponds to the angle uncertainty, i.e. $\Delta k / k=\Delta \phi$. The chosen value yields typical values of $\sigma \approx 8 \mathrm{~nm}$ for small energies, while for higher energies $\sigma$ can be smaller than $4 \mathrm{~nm}$. In the following and for device A the notation $x=\mathrm{n}$ will denote a cut in the $\mathrm{n}$ region of the device, $3 \sigma$ before the $\mathrm{p}-\mathrm{n}$ junction, while $x=\mathrm{p}$ denote a cut $3 \sigma$ after the junction. For device $\mathrm{B}$ the slice location is given explicitly (in the rest of the text we measure space in $\mathrm{nm}$ and energy in $\mathrm{eV}$ ).

\section{APPLICATIONS OF THE HUSIMI FUNCTION}

\section{A. Accuracy of the Husimi function}

We first want to test the usage of $Q$ in a well studied situation where much can be inferred analytically: Klein tunneling in device A at small energies ${ }^{28}$ ( $\mathrm{see}^{35}$ for a review on Klein tunneling in graphene). Fig. 2(b-e) shows $Q(\phi, y)$ in device A for $W=80, L=12 \sigma \approx 96, E_{F}=$ 
$0, V_{0}=0.4$. The top panels show $Q$ for valley $K_{2}$, the bottom for $K_{2}^{\prime}$. We show $Q$ both before (incoming \& reflected) and after (outgoing) the p-n junction for two modes. What we have seen is that for device A before the junction, $Q$ in valley $K_{2}\left(^{\prime}\right)$ is the mirror reflection of $Q$ in valley $K_{3}\left({ }^{\prime}\right)$ while in valley $K_{1}\left({ }^{\prime}\right)$ we find an almost exact superposition of the $Q \mathrm{~s}$ in $K_{1}\left({ }^{\prime}\right)$ and $K_{2}\left(^{\prime}\right)$.

Fig. 2 shows that for all modes the incoming $Q$ nicely localizes at a single angle. We also show in red the marginal distributions

$$
\begin{aligned}
Q(\phi ; \xi) & =\int_{0}^{W} Q(\phi, y ; x, \xi) d y \\
Q(\phi) & =\sum_{\xi} Q(\phi ; x, \xi)
\end{aligned}
$$

Because in this setup the incoming $Q$ is very highly localized, we do not need the entire distribution and can simply choose the maximum location of $Q(\phi), \Phi$, to represent the "incoming angle" for each mode

$$
\Phi=\operatorname{argmax}[Q(\phi ; x=\mathrm{p})], \text { for } \phi \in\left[0, \frac{\pi}{2}\right)
$$

(we use $Q$ of valley $K_{2}\left({ }^{\prime}\right)$ exclusively for this, and we also know which of the two valleys is the incoming one).

We compare $\Phi$ with $\nu$, the angle obtained by sinefitting the lead modes, in Fig. 3a. We see that only for the lowest modes of each cone $\Phi$ does not have a perfect agreement with $\nu$. We now want to use $\Phi$ to compare the results of the tight binding calculations with theoretical result for the Klein tunneling at a p-n junction, utilizing the impact angle obtained from the Husimi function. Depending on the width of the $\mathrm{p}-\mathrm{n}$ junction, there are two theoretical predictions

$$
\begin{aligned}
T_{\text {Step }}\left(\phi_{\text {in }}\right) & =-\frac{\cos \left(\phi_{\text {in }}\right) \cos \left(\phi_{\text {out }}\right)}{\sin ^{2}\left(\frac{1}{2}\left(\phi_{\text {in }}+\phi_{\text {out }}\right)\right)} \\
T_{\mathrm{WKB}}\left(\phi_{\text {in }}\right) & =\exp \left(-\pi \frac{2 k_{1}^{2}}{k_{1}+k_{2}} \frac{w}{2} \sin ^{2} \phi_{\text {in }}\right),
\end{aligned}
$$

where $\phi_{\text {in }}\left(\phi_{\text {out }}\right)$ is the wavevector angle of the incoming and transmitted wave, respectively, and $k_{j}=\mid E_{F} \pm$ $V_{0} / 2 \mid /\left(\hbar v_{F}\right)$ the corresponding wavenumber. $T_{\text {Step }}$ is the result of wave function matching at a sharp interface whereas $T_{\mathrm{WKB}}$ is a semiclassical result obtained in the WKB approximation ${ }^{35,36}$. In Fig. 3c we plot the theoretical curves and the values of $T_{m}$ versus $\Phi$ for each mode, for two different $\mathrm{p}-\mathrm{n}$ junction widths $w$, and find very good agreement. This does not only hold for the case of a symmetric p-n junction, i.e. $E_{F}=0$, but also for higher and lower Fermi energies, as shown in Fig. 3d for $w=10 \mathrm{~nm}$ (for other parameter values we also find excellent agreement).

We have seen, that we can use $Q$ to find the parameter $\phi_{\text {in }}$ from numerical simulations needed to compare them to the theoretical predictions. Now we want to show that we can obtain the transmission probabilities from the Husimi function using the theoretical predictions. Using the marginal distribution of eq. (7) we can compute the transmission of a mode as the average

$$
\langle T\rangle=\frac{\int_{-\frac{\pi}{2}}^{\frac{\pi}{2}} T(\phi) Q(\phi ; x=\mathrm{p}) d \phi}{\int_{-\frac{\pi}{2}}^{\frac{\pi}{2}} Q(\phi ; x=\mathrm{p}) d \phi}
$$

where $T$ represents one of the analytical formulas of eqs. (9) and (10). In Fig. 3b we compare this value with $T_{m}$ and again we find a near perfect match (also for many more parameters than the ones shown). Equation (11) will also give a good estimate of the transmission value in cases where the distribution is not strongly localized at a single angle, allowing us to use the integrated transmission in more complicated cases like those in sec. II C.

\section{B. Intervalley Scattering}

We now turn to study intervalley scattering, which describes the scattering of a wavefunction from one valley to another (inequivalent) one, e.g. from $K$ to $K^{\prime}$. We discussed in sec. IB that for zigzag GNRs and low energies every incoming mode is valley-polarized ${ }^{28}$. Intervalley scattering has found considerable interest in the literature, and was first discussed in the context of weak localization $^{2,37-39}$. Later work focused on valley filters and valley "spintronics", see ${ }^{40-42}$ and references therein. The discussions in the literature so far have been qualitative and mostly theoretical.

The Husimi function is an excellent tool to study intervalley scattering, because it directly provides information in momentum space at different positions in the device. In fact, Mason et al. have used a processed Husimi projection technique in Ref. ${ }^{22}$ to study intervalley scattering in graphene billiards. Here we will use a simpler approach directly using the Husimi function. As one can already see from Fig. 2b-e, the "incoming $Q$ " (i.e. $Q(y, \phi)$ with $\phi \in[-\pi / 2, \pi / 2)$ ) has most weight in one valley (the "incoming valley") $V_{i}$, while the other (the "complementary") valley $V_{c}$ contains either just noise or only the reflected wave (compare the scales of the colorbars). In panels (c, e) it is evident there exist modes that undergo intervalley scattering, as for panel (e) the outgoing valley $K^{\prime}$ has significantly more weight than what it had in the incoming case of panel $(d)$.

We want to define two intuitive measures for intervalley scattering. We first define the following weights (the sums are over all equivalent valleys)

$$
\begin{aligned}
\alpha & =\sum_{\xi \in V_{i}} \int_{0}^{W} \int_{-\frac{\pi}{2}}^{\frac{\pi}{2}} Q(\phi, y ; x=\mathrm{n}, \xi) d \phi d y \\
\beta & =\sum_{\xi \in V_{i}} \int_{0}^{W} \int_{-\pi}^{\pi} Q(\phi, y ; x=\mathrm{p}, \xi) d \phi d y \\
\gamma & =\sum_{\xi \in V_{c}} \int_{0}^{W} \int_{-\pi}^{\pi} Q(\phi, y ; x=\mathrm{p}, \xi) d \phi d y .
\end{aligned}
$$



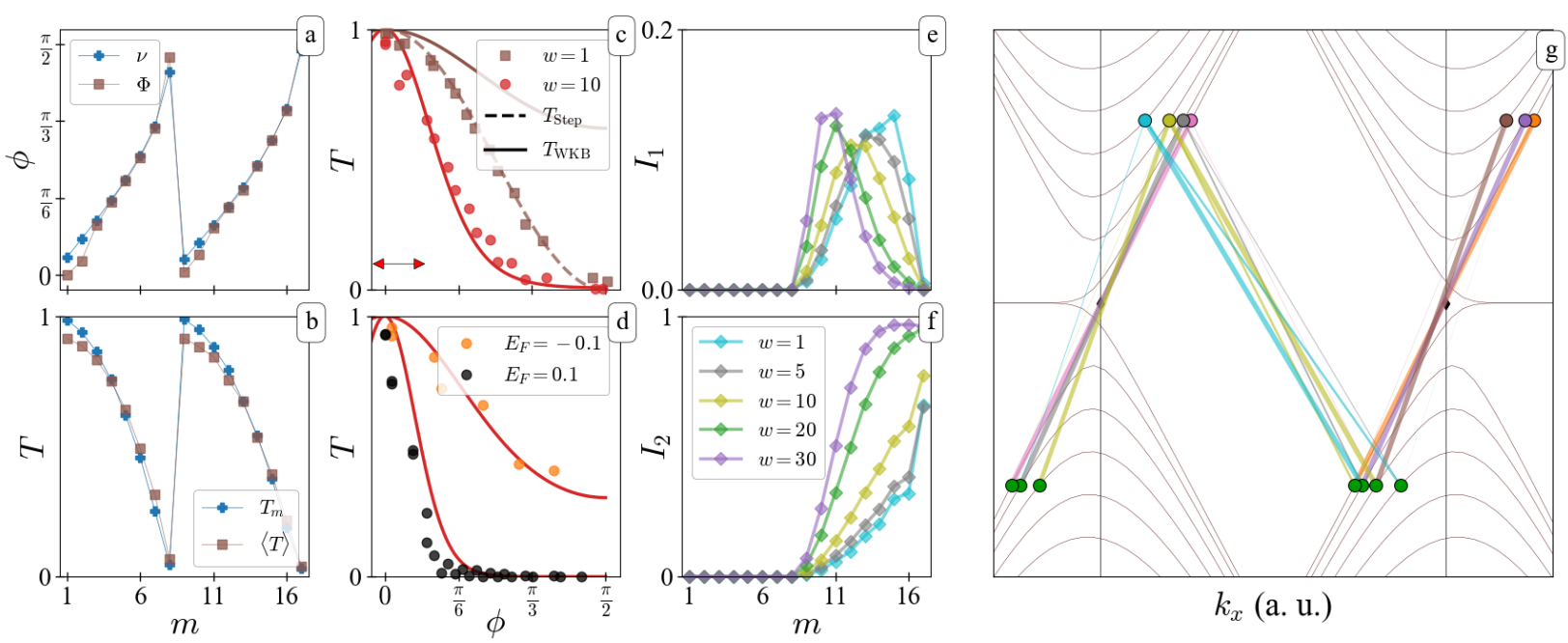

FIG. 3. Klein tunneling and intervalley scattering in device $\mathrm{A}$ for $\sigma=8, W=80, L=12 \sigma, V_{0}=0.4, E_{F}=0, \pm 0.1$ and various $w$. (a) Angle of incidence $\nu$ deduced from the transverse wavefunctions, compared with the ones deduced from the Husimi function, $\Phi$. (b) Transmission probability obtained through the scattering matrix $T_{m}$ eq. (5) versus the integrated one obtained from $Q,\langle T\rangle$ using eq. (11). (a, b) are plotted versus incoming mode $m$ and for $w=1 \mathrm{~nm}$. (c) Theoretical curves on Klein tunneling (lines, eqs. (10), (9)) and transmission probability $T_{m}$ versus $\Phi$ (scatter plots, for two different $w$ values). The red arrow notes the angle uncertainty $\Delta \phi$. (a, b, c) use $E_{F}=0 \mathrm{eV}$. (d) is the same as (c) but for $w=10$ and different Fermi energies instead. (e,f) Measures for intervalley scattering, eq. (15) versus mode number. (g) Sketch (x-axis is not uniform) of where is each mode transmitted, based on the elements of the transmission matrix $\mathcal{T}$. The width of each line is proportionate to the transmission amplitude towards the outgoing channel that the line connects to (normalized to same maximum).

The first quantity, $\alpha$, is used for the normalization to the incoming mode. The quantities $\beta$ and $\gamma$ measure the weights of the transmitted wave that are localized in the same valley as the incoming mode and its complement, respectively. With these quantities we define

$$
I_{1}=\frac{\gamma}{\alpha}, \quad I_{2}=\frac{\gamma}{\beta+\gamma} .
$$

Here $I_{1}$ is the the fraction of the incoming wave that is transmitted through the $\mathrm{p}-\mathrm{n}$ junctions and has undergone intervalley scattering. $I_{2}$ is the fraction of the transmitted wave that has undergone intervalley scattering, i.e. a transmitted wave with $I_{2}=0$ or $I_{2}=1$ is completely valley polarized. We show both measures of intervalley scattering in Fig. 3e,f plotted versus the mode number for various junction widths. (Qualitatively the results remain unchanged when we use only $K_{2}\left({ }^{\prime}\right)$ instead of summing over equivalent valleys).

The most striking feature of Fig. 3e,f is that intervalley scattering happens only for the second half of the modes. Recall that modes with $1 \leq m \leq\lfloor M / 2\rfloor$ come from $K^{\prime}$ while the higher modes come from the $K$ valley which has an additional incoming band (see Fig. 1a or Fig. 3g). The perplexing result of Fig. 3e can be qualitatively explained based on this extra mode and the unitarity of the scattering matrix $S^{32}$ (i.e. current conservation). To aid the following argument, in Fig. $3 \mathrm{~g}$ we show a sketch of where is each incoming mode transmitted. The lines connecting incoming and outgoing modes have widths directly proportional to the transmission amplitude $\left|\mathcal{T}_{i m}\right|^{2}$.
After transmission, each mode "tries" to scatter into a the same valley at negative energy to conserve the valley pseudospin (green dots in Fig. 3g). Likewise should the reflected part scatter into modes in the same valley at the same energy level but with negative group velocity. Modes 1 to $\lfloor M / 2\rfloor$ have no problem achieving this, as within their valley the outgoing channels are more than the incoming ones and thus available channels always exist. This is not the case however for modes $\lfloor M / 2\rfloor+1$ to $M$, since the number of outgoing channels within the same valley is one less, both for transmission and reflection. As the mode number increases the outgoing channels are filled and the higher modes have to move some of their weight to other channels (as a specific outgoing channel cannot be filled with more than total transmission of 1 , see Ref. ${ }^{32}$ ). The only remaining channels that can accommodate these modes exist in the $K^{\prime}$ valley (right valley of Fig. 1a) which leads to intervalley scattering. This qualitative argument cannot certainly explain the exact values of intervalley scattering, but gives a basic idea of the phenomenon.

Looking at $I_{1}$ we see a complicated dependence on the junction width $w$. For modes with small angle of incidence $I_{1}$ increases as $w$ increases, however the contrary happens for modes with large angle of incidence. $I_{1}$ always tends to 0 as $m \rightarrow M$ since the highest mode has almost 0 transmission. Another interesting observation is that as mode number (and thus angle of incidence) is increased, the modes that do get intervalley scattered transfer most of their weight into the other valley. This 
can be seen from Fig. 3f where $I_{2}$ comes close to 1 .

\section{Asymmetric Device}

In this section we study transport through the asymmetric device B (see Fig. 1c) in which the incoming modes are scattered both from the boundary ("scattering edge", highlighted in green) and the p-n junction, and which is neither axially nor centrally symmetric. There are two main questions we want to address. First, to what extend can we use the existing expressions describing Klein tunneling to understand the transmission properties of such a device? These expressions are derived for plane waves, which have infinite spatial extend and are characterized by a single angle $\phi_{\text {in }}$, either semiclassically or by wave matching. Due to the boundary induced scattering the wavefunction that incides on the $\mathrm{p}-\mathrm{n}$ junction in device $\mathrm{B}$ cannot be well approximated by a single plane wave. Can we use the Husimi technique to connect the transmission through the device to Klein tunneling?

Second, we want to understand how the type of the scattering boundary (green color in Fig. 1c) affects intervalley scattering. There is strong theoretical evidence that the armchair termination is in some way unique, while a random termination behaves like zigzag ${ }^{29,43,44}$. In addition, in the theoretical treatment of graphene nanoribbons $\mathrm{in}^{28}$, the authors showed that the armchair termination mixes valleys while the zigzag keeps them separated.

These arguments indicate that intervalley scattering should be enhanced by an edge with armchair termination. However, they are only qualitative. Mason et al. have shown $i^{21}$ that a (also Husimi-based) qualitative measure of intervalley scattering is generally enhanced at armchair boundaries. Here we want to quantify of these effect by using the Husimi function, similarly as in II B and we will show that intervalley scattering is indeed enhanced drastically at armchair edges.

Let us stress that in device B the lead modes and thus the angles $\nu$ are not of much use. This is in part because the waves are deflected by the titled boundary of device B, but also because the right part of device B has a different width than the incoming lead (and thus does not accommodate the same $k_{y}$ ). On the other side, $Q(\phi)$ is just as valid here as it was in sec. II A. It also becomes clear from Fig. 4a that many of the scattering waves inside $L_{2}$ cannot be approximated using a single angle, which means that one needs the entire distribution.

\section{Tunneling}

We find that we can apply the Klein tunneling formulas "locally" even in small devices and when the incoming waves are not single plane waves. We show this numerically using the integrated transmission formula, eq. (11) with $Q$ measured at location $x=L_{1}+L_{2} / 2$ (which is $3 \sigma$ before the p-n junction). However, now we can't compare $\langle T\rangle$ with $T_{m}$ directly, because $T_{m}$ also accounts for the back-scattering from the boundary inside $L_{1}$. To compensate for that, we compute the transmission of eq. (5) once without any p-n junction at all. We call this quantity $T_{0}$. We now have to compare $\langle T\rangle \cdot T_{0}$ with $T_{m}$, which we do in Fig. 4c-f for various orientations of the boundary.

We see that the integrated transmission matches the transmission obtained through the $\mathrm{p}-\mathrm{n}$ junction (using the scattering matrix) very well. This good agreement means that the Klein tunneling formula still locally describes the tunneling properties at the p-n junction, even when the nanodevice is small (e.g. $60 \mathrm{~nm}$ for $\omega=\pi / 4$ ) and the incoming wave is not a simple plane wave. In addition, this also means that the Husimi function accurately decomposed the incoming scattering wave into a representative distribution of angles of incidence.

\section{Intervalley scattering}

We now want to explore the intervalley scattering induced by the scattering edge and not the p-n junction. Therefore we first obtain the scattering wavefunctions $\psi_{m}$ in device B without a p-n junction (i.e. $V_{0}=0, E_{F}=0.2$ $\mathrm{eV}$ ). We measure $Q$ using a slice at $x=L_{1}$ (exactly where the scattering boundary ends) and we compute $I_{2}$ from $Q$ there. The results are shown in Fig. $4 \mathrm{~g}$-i.

An important benefit of using $I_{2}$ (over e.g. measures used $\mathrm{in}^{21}$ ) is that it does not depend on, or demand measuring $Q$ for $\mathbf{r}_{0}$ exactly at the boundaries. This is crucial as the accuracy of the Husimi function dramatically drops at the boundaries, since most lattice sites around a circle of $3 \sigma$ from $\mathbf{r}_{0}$ do not even exist. We have observed in our simulations that this leads to numeric artifacts and should be avoided (this is also clear from all colorplots of $Q$ we show in this paper, see e.g. Fig. 2 or 4 where the value of $Q$ drastically drops for $y \rightarrow 0$ or $y \rightarrow W)$.

There are two interesting observations to be made. First, the intervalley scattering from a lattice termination is fundamentally different from that seen in sec. II B which results from a p-n junction. In the present case both valleys always undergo intervalley scattering.

The second observation is what we expected from existing theory and now quantified using a well-defined measure: armchair lattice terminations induce much more intervalley scattering than any other termination orientation. This can be seen firstly in Fig. $4 \mathrm{~g}$, h where $I_{2}$ has clearly higher values, but most prominently in panel $\mathrm{i}$ where we plot the average intervalley scattering per mode, i.e.

$$
\tilde{I}_{2}=\frac{1}{M} \sum_{m}^{M} I_{2}(m) .
$$

$\tilde{I}_{2}$ has a very sharp peak at $\omega=\pi / 6$, where the boundary 

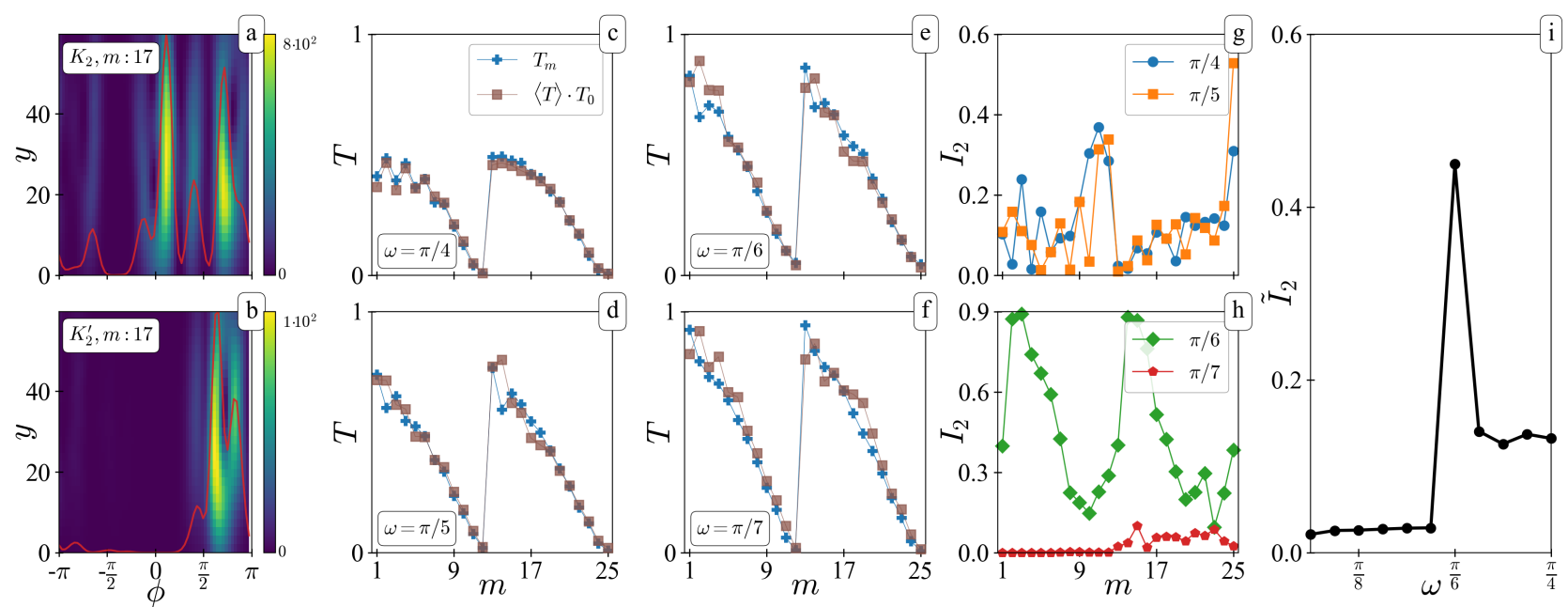

FIG. 4. Tunneling and intervalley scattering in device B, using $\sigma=10, L_{1}=L_{2}=6 \sigma, W_{1}=120, w=1, V_{0}=0.4, E_{F}=0$.

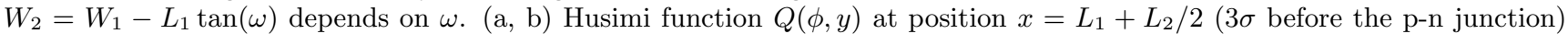
for $\omega=\pi / 4$. For the mode shown, the incoming valley is $K_{2}$ (but a lot of intervalley scattering has already occurred). (c-f) Integrated transmission. ( $\mathrm{g}, \mathrm{h}$ ) Intervalley scattering $I_{2}$ of eq. (15) for various $\omega$ values using $Q$ measured at $x=L_{1}$ (computed without a p-n junction, $V_{0}=0, E_{F}=0.2$ ). (i) Average intervalley scattering per mode $\tilde{I}_{2}$ versus boundary angle $\omega$. A sharp increase is seen when $\omega=\pi / 6$.

termination is exactly armchair.

\section{Trigonal Warping and Klein Tunneling}

Klein tunneling applies to graphene because for small energies the Dirac equation is a valid approximation. In Klein tunneling the important angle is the wavevector angle (with respect to the Dirac points), see eqs. (9), (10). The group velocity angle $\theta$ coincides with $\phi$ for small energies, however as the energy increases and trigonal warping effects begin to be significant, this is not the case anymore and $\theta \neq \phi^{5}$. As there is no theoretical result on the tunneling behavior of graphene for energies beyond the Dirac regime, one is left to wonder: for higher energies is the Klein tunneling picture still relevant? And if yes, are the tunneling properties still dictated by $\phi$ ? This is an interesting question since the physical propagation direction is governed by $\theta$.

We can answer this using the Husimi function. We return to our setup in device A like in sec. II A but significantly increase the energies, setting $V_{0}=5$ and keeping $E_{F}=0$, yielding incoming energy of $E=2.5 \approx 0.9 t$ which shows strong trigonal warping. Once again we compute incoming angles using $\Phi$ as in eq. (8) because incoming $Q$ is well-localized in momentum space, see fig. 5a,b. However, the limits of argmax must be modified. For modes $m \leq\lfloor M / 2\rfloor$ the angle span of eq. (8) is set to $\left[0, \frac{\pi}{3}\right)$ while for the rest of the modes it is set to $\left[0, \frac{2 \pi}{3}\right)$, due to the warping of the energy contour, see below.

In this energy regime the theory of Brey and Fertig 28 breaks down and the transverse wavefunctions are not necessarily sin-functions. One may still attempt to fit sines to them, as shown in Fig. $5 \mathrm{~d}$ and e, however here we find $Q$ to perform better, as shown in panel (d). So even though $k_{x}$ is still known, one cannot straight forwardly compute $k_{y}$ using only the lead modes.

At higher energies the two valleys are very different, since the group velocities of the incoming modes differ fundamentally (see Fig. 5c). For valley $K^{\prime}$ there is a "flat" front, greatly limiting the possible group velocities. The contrary is happening in valley $K$ where the contour with positive group velocity spans many more angles. In addition, in the $K^{\prime}$ case the incoming wavevector angle is limited to $\left|\phi_{\text {in }}\right| \lesssim \pi / 3$ but in $K$ we have $\left|\phi_{\text {in }}\right| \lesssim 2 \pi / 3$, due to the requirement of positive $\mathrm{x}$-component of the group velocity, calculated from eq. (6)

$$
\begin{aligned}
& v_{x}=\frac{-\sqrt{3} \lambda t a}{\sqrt{f(k)+3}}\left(\sin \left(\frac{\sqrt{3} a}{2} k_{x}\right) \cos \left(\frac{3 a}{2} k_{y}\right)+\sin \left(\sqrt{3} k_{x} a\right)\right) \\
& v_{y}=\frac{-3 \lambda a}{\sqrt{f(k)+3}} \cos \left(\frac{\sqrt{3} a}{2} k_{x}\right) \sin \left(\frac{3 a}{2} k_{y}\right) .
\end{aligned}
$$

Here $k_{x}, k_{y}$ are measured with respect to the center of the BZ since the above equations are valid for any energy value.

Klein tunneling assumes equivalence between the two valleys as it depends on the wavevector angle. To see whether some remnant of Klein tunneling exists at higher energies, we have to look for some tunneling property that not only decays exponentially with increasing angle of incidence, but also stays "as similar" as possible between the two valleys. In fig. 5 d,e we compare the trans- 

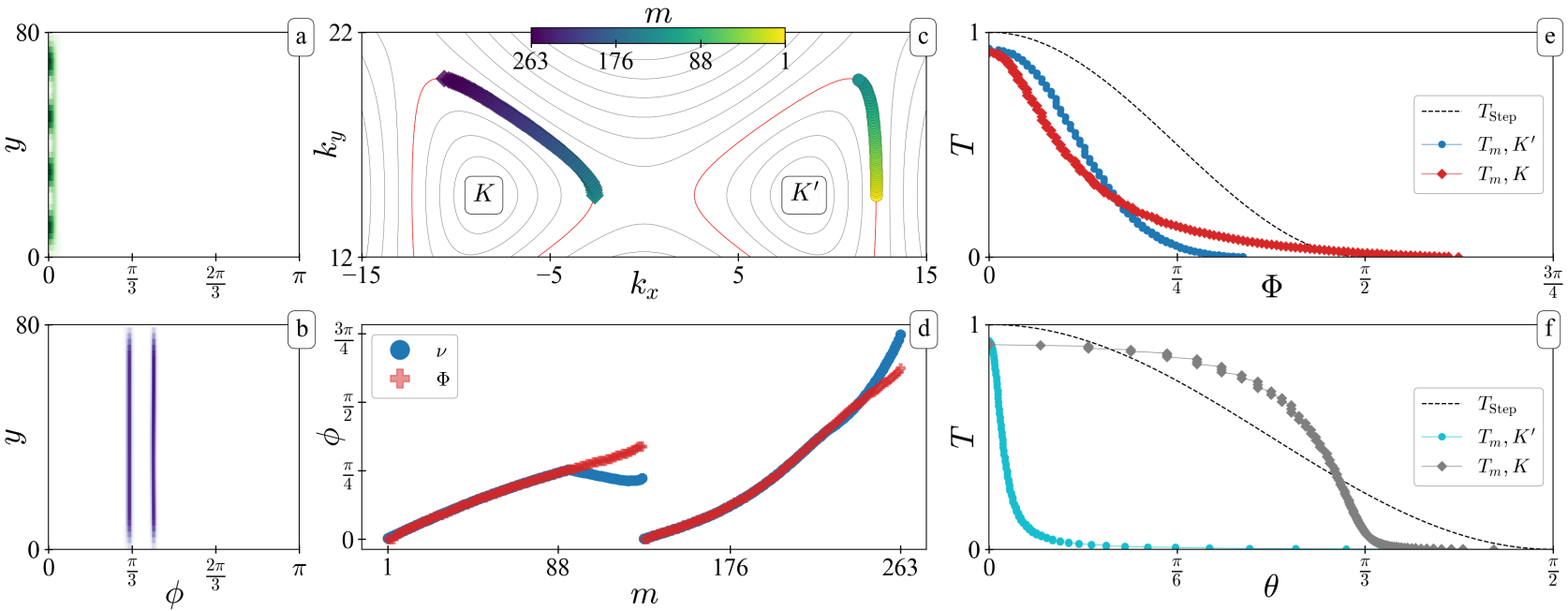

FIG. 5. Transmission and Husimi functions in device A for high energies: $\sigma=4, L=12 \sigma, W=90, V_{0}=5, E_{F}=0, w=1$. (a, b) $Q(\phi, y)$ for the modes shown in (e). (c) Maxima of incoming $Q$ (see sec. IID) on momentum space. Each incoming mode is using a different color, see colorbar. (d) Angles deduced through sine fitting $(\nu)$ versus the ones deduced from $Q$ $(\Phi)$. (e) Transverse wavefunction amplitude $Y$ on sublattice $A$ (arbitrary choice) versus $y$ inside the lead. Small modes can be approximated by sines but higher modes cannot. (f) Mode transmission $T_{m}$ versus wavevector angle (obtained using the Husimi function). (g) Same but versus group velocity angle $\theta$ instead. The dashed line plot of $T_{\text {Step }}$ is only meant as a guide to the eye, the formula is not valid for high energies.

mission probability of each mode $T_{m}$ versus the wavevector angle $\Phi$ and group velocity angle $\theta$.

The result surprised us, since we find a Klein tunnelinglike behaviour in $T_{m}$ versus $\phi$. We were rather expecting $T_{m}$ versus $\theta$ to show similar behaviour at the two valleys, because $\theta$ corresponds to the physical propagation direction. We do not suggest that Klein tunneling straightforwardly applies to higher energies. In Fig. $5 \mathrm{f}$ the characteristic perfect transmission at normal incidence $\left(\phi_{\text {in }}=0\right)$ is lost, nevertheless, it is clear that the tunneling probability as a function of the wavevector angle is quite similar to what would be expected for Klein tunneling.

\section{E. From Dirac points to the center of the Brillouin zone}

Before concluding our paper we want to show some interesting numerical results for the case of high but not symmetric energies. Here we use the setup exactly as in section IID but we set $E_{F}= \pm 1 \mathrm{eV}$. In this case the incoming (outgoing) modes, for plus (minus) sign, do not live in the trigonally warped Dirac points, but instead are located on the almost circular contours around the center of the BZ, see Fig. 6. In this case any Klein tunellinglike behaviour is impossible as there is no Dirac valley in either the incoming or outgoing modes. This of course does not exclude an angle collimation effect in the transmission function, it only excludes Klein tunneling as a potential explanation.

Once again we are interested in $T_{m}$ versus some incom-
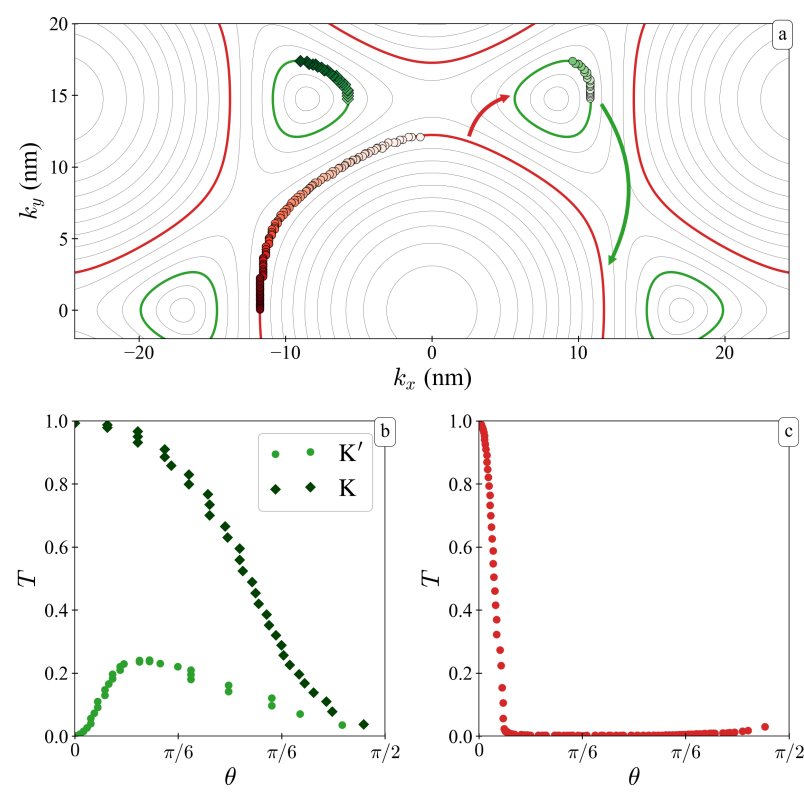

FIG. 6. Tunneling through a pn-junction for high and nonsymmetric energies. (a) Incoming modes, as identified using the Husimi function, for high energy (red, $E_{F}=1$ ) or low (green, $E_{F}=-1$ ) for $V_{0} / 2=5$. Arrows guide the eye for where is each contour transmitted to (of course in the negative energy space). In (b, c) we plot the transmission of modes incoming from the green or red contour respectively, versus their group velocity angle. 
ing angle. However, it does not make sense to measure angles from the nearby Dirac centers. Instead, for all incoming modes we use the Husimi function to identify the wavevectors $k_{x}, k_{y}$ where the mode is localized, from the entire BZ (as in Fig. 2). To be able to compare across the different energy regimes, we then calculate the angle of the group velocity $\theta$ at point $k_{x}, k_{y}$.

In Fig. 6a we plot the points where the Husimi function is localized for each incoming mode. Then, in subplots $b$ and $\mathrm{c}$, we show the transmission versus angle of incidence for the case of low to high (absolute value in) energy (green) and high to low (red). A very surprising result is shown in (b): the transmission for one incoming valley is much higher. Not only that, but for valley $K^{\prime}$ the transmission goes to 0 for $\theta \rightarrow 0$. For the transmission from high to low energy (red) we see a very quick drop of the transmission as $\theta$ increases. What is noteworthy (and quite perplexing) is that the transmission increases again when $\theta$ approaches $\pi / 2$.

\section{CONCLUSION}

In conclusion we have found that the Husimi function can indeed be a very useful tool for studying transport in graphene (but not only graphene) nanostructures. We have for example shown that even in situations where the angle of incidence on a tunnel barrier is not easily discernible we can use the Husimi distribution to evaluate Klein tunneling at this barrier. For higher Fermi energies, we have studied the tunneling behavior in the regime of triangular warped Dirac cones. Specifically we looked at two cases: tunneling between warped cones and tunneling between states near the center of the Brillouin zone and the warped Dirac cones. In the first case, overall the behavior we found is clearly different from Klein tunneling between Dirac cones. The transmission probability as a function of angle of incidence varies when the angle is either measured by the directions of the wave vectors or the group velocities. When measured by the wave vector, however, we observed reminiscences of Klein tunneling. In the second case we found a strong asymmetry between the two valleys. Other interesting results concern intervalley scattering. At a pn-junction in a nanoribbon we found a pronounced valley asymmetry of the intervalley scattering due to the extra mode in the dispersion relation of the nanoribbon. We have also quantified the intervalley scattering at a tilted graphene edge as the function of the tilt angle, confirming the special role played by the armchair boundary configuration.

\section{Appendix A: Angle uncertainty}

For a given value of the parameter $\sigma$, the wavepacket has a known uncertainty in both position and momentum

$$
\sigma:=\Delta x=\frac{1}{2 \Delta k} .
$$

What we are interested about is the uncertainty in the propagation angle. For small energies the propagation angle is the same for the wavevector and the group velocity defined as

$$
\phi=\arctan \left(q_{y} / q_{x}\right)
$$

with $\mathbf{q}=\mathbf{k}-K^{\xi}$. For any nonlinear function, uncertainty propagation is given by

$$
\sigma_{\phi}^{2}=\left|\frac{\partial \phi}{\partial q_{x}} \sigma_{q_{x}}\right|^{2}+\left|\frac{\partial \phi}{\partial q_{y}} \sigma_{q_{y}}\right|^{2} .
$$

Since by definition $\sigma_{q_{x}}=\sigma_{q_{y}}=\Delta q=\Delta k$ we have

$$
\sigma_{\phi}^{2}=\frac{q_{y}^{2} \Delta q^{2}}{\left(q_{x}^{2}+q_{y}^{2}\right)^{2}}+\frac{q_{x}^{2} \Delta q^{2}}{\left(q_{x}^{2}+q_{y}^{2}\right)^{2}}=\Delta q^{2} \frac{q^{2}}{q^{4}}
$$

therefore we see that

$$
\sigma_{\phi}=\frac{\Delta q}{q}
$$

If we want to have a constant $\sigma_{\phi}$ for measurements at different energies, then we will use $\sigma$ such that (assuming also $\Delta x \Delta k=1 / 2)$

$$
\frac{\Delta q}{q}=\frac{1}{2 q \sigma} \Rightarrow \sigma=\frac{1}{2 q}\left(\frac{\Delta q}{q}\right)^{-1} \Rightarrow \sigma=\frac{1}{2 \sigma_{\phi} q} .
$$

${ }^{1}$ K. S. Novoselov, Z. Jiang, Y. Zhang, S. V. Morozov, H. L. Stormer, U. Zeitler, J. C. Maan, G. S. Boebinger, P. Kim, and A. K. Geim, Science 315, 1379 (2007).

${ }^{2}$ E. McCann, K. Kechedzhi, V. I. Fal'ko, H. Suzuura, T. Ando, and B. L. Altshuler, Physical Review Letters 97, 146805 (2006).

${ }^{3}$ M. I. Katsnelson, K. S. Novoselov, and a. K. Geim, Nature physics 2, 15 (2006), arXiv:0604323 [cond-mat].

4 J. Tworzydło, B. Trauzettel, M. Titov, A. Rycerz, and C. W. J. Beenakker, Phys. Rev. Lett. 96, 246802 (2006).

${ }^{5}$ S. Das Sarma, S. Adam, E. H. Hwang, and E. Rossi, Reviews of Modern Physics 83, 407 (2011), arXiv:1003.4731.
${ }^{6}$ K. Husimi, Proceedings of the Physico-Mathematical Society of Japan. 3rd Series 22, 264 (1940).

7 W. P. Schleich, Quantum Optics in Phase Space (2001).

8 H. Moya-Cessa, J. R. Moya-Cessa, and L. R. BerrielValdos, Introduction to quantum optics , 31 (2008).

9 A. L. Virovlyansky, D. V. Makarov, and S. V. Prants, , 30 (2012).

10 S. Nonnenmacher and A. Voros, Journal of Statistical Physics 92, 431 (1998).

11 A. Bcker, in The Mathematical Aspects of Quantum Maps, edited by M. D. Esposti and S. Graffi (Springer Berlin Heidelberg, Berlin, Heidelberg, 2003) pp. 91-144. 
12 A. Bcker, S. Frstberger, and R. Schubert, Physical Review E 70, 036204 (2004).

13 A. Bcker, R. Ketzmerick, and A. G. Monastra, Physical Review Letters 94, 054102 (2005).

${ }^{14}$ F. Toscano, A. Kenfack, A. R. Carvalho, J. M. Rost, and A. M. Ozorio de Almeida, Proceedings of the Royal Society A: Mathematical, Physical and Engineering Sciences 464 1503 (2008).

15 H. Schanz, T. Dittrich, and R. Ketzmerick, Physical Review E 71, 026228 (2005).

${ }^{16}$ K. W. Mahmud, H. Perry, and W. P. Reinhardt, Physical Review A 71, 023615 (2005).

17 M. Hentschel, H. Schomerus, and R. Schubert, EPL (Europhysics Letters) 62, 636 (2003).

18 J. Wiersig and M. Hentschel, Physical Review Letters 100 , 033901 (2008).

19 A. Bcker, R. Ketzmerick, S. Lck, J. Wiersig, and M. Hentschel, Physical Review A 79, 063804 (2009).

20 J. Feist, A. Bcker, R. Ketzmerick, S. Rotter, B. Huckestein, and J. Burgdrfer, Physical Review Letters 97 116804 (2006)

${ }^{21}$ D. J. Mason, M. F. Borunda, and E. J. Heller, Physical Review B - Condensed Matter and Materials Physics 88 1 (2013).

22 D. Mason, M. Borunda, and E. Heller, EPL (Europhysics Letters) 60005, 1 (2013), arXiv:arXiv:1205.0291v3.

23 D. J. Mason, M. F. Borunda, and E. J. Heller, Physical Review B 91, 165405 (2015).

${ }^{24}$ K. Takahashi, Journal of the Physical Society of Japan 55 , 762 (1986)

25 J. E. Harriman, The Journal of Chemical Physics 88, 6399 (1988).

${ }^{26}$ E. J. Heller, The Semiclassical Way to Dynamics and Spectroscopy (Princeton University Press, 2018).

27 For each $\mathbf{r}_{0}$ we use only lattice sites that are within $\left|\mathbf{r}_{j}-\mathbf{r}_{0}\right| \leq 3 \sigma$, to reduce computation time. Notice the complex conjugation $\psi^{*}$ in eqs. (3) and (4), which sometimes is omitted in the literature. While for closed systems with time-reversal symmetry it can be omitted, it is crucial for open systems, which we explore here, and for systems with broken time reversal symmetry. This can be trivially seen by computing the Husimi function of a plane wave $\psi=\exp (i \mathbf{k} \cdot \mathbf{r})$ which is solvable analytically, giving $Q \propto \exp \left(\left(\mathbf{k}_{0}-\mathbf{k}\right)^{2}\right)$. Without the complex conjugation the result would be $\propto \exp \left(\left(\mathbf{k}_{0}+\mathbf{k}\right)^{2}\right)$ which is of course wrong, as $Q$ should have a maximum at $\mathbf{k}$ and not $-\mathbf{k}$.

${ }^{28}$ L. Brey and H. a. Fertig, Physical Review B - Condensed Matter and Materials Physics 73, 2 (2006), arXiv:0603107 [cond-mat].

29 A. R. Akhmerov and C. W. J. Beenakker, Physical Review B 77, 085423 (2008).

${ }^{30}$ C. W. Groth, M. Wimmer, A. R. Akhmerov, and X. Waintal, New Journal of Physics 16, 1 (2014), arXiv:1309.2926.

${ }^{31}$ We note that this is stationary, not a time-dependent description.

32 S. Datta, Electronic Transport in Mesoscopic Systems (Cambridge University press, 1995).

33 a. H. Castro Neto, F. Guinea, N. M. R. Peres, K. S. Novoselov, and a. K. Geim, Reviews of Modern Physics 81, 109 (2009), arXiv:0709.1163.

${ }^{34}$ M. O. Goerbig, Rev. Mod. Phys. 83, 1193 (2011), arXiv:1004.3396.

35 P. E. Allain and J. N. Fuchs, European Physical Journal B 83, 301 (2011), arXiv:1104.5632.
${ }^{36}$ V. V. Cheianov and V. I. Fal'ko, Physical Review B 74 041403 (2006), arXiv:0603624 [cond-mat].

37 A. F. Morpurgo and F. Guinea, Physical Review Letters 97, 1 (2006), arXiv:0603789 [cond-mat].

38 M. Hilke, M. Massicotte, E. Whiteway, and V. Yu, TheScientificWorldJournal 2014, $737296 \quad$ (2014), arXiv:arXiv:1212.5334v1.

39 B. Yan, Q. Han, Z. Jia, J. Niu, T. Cai, D. Yu, and X. Wu, Physical Review B 93, 1 (2016), arXiv:1602.06519.

40 A. Rycerz, J. Tworzydło, and C. W. Beenakker, Nature Physics 3, 172 (2007), arXiv:0608533 [cond-mat].

41 D. Gunlycke and C. T. White, Physical Review Letters 106, 1 (2011), arXiv:1103.4313.

42 K. W. Lee and C. E. Lee, Physical Review B 95, 1 (2017).

43 E. McCann and V. I. Fal ko, Journal of Physics: Condensed Matter 16, 2371 (2004).

44 J. A. Van Ostaay, A. R. Akhmerov, C. W. Beenakker, and M. Wimmer, Physical Review B - Condensed Matter and Materials Physics 84, 1 (2011), arXiv:1109.0884. 


\section{Husimi functions and Klein tun- neling in magnetic fields}

This chapter continues from chapter 6 and studies Klein tunneling using the Husimi function but now with the presence of a perpendicular magnetic field. As it will become clear below, this entails differences, both for using the Husimi function, but also for studying Klein tunneling.

The first big difference is that it seems that Klein tunneling in magnetic fields is not fully understood, in contrast with the absence of magnetic fields, where well-established analytic expressions exist. To see why, first consider the influential publication in Phys. Rev. Lett. by Shytov et al [144, 145] and the subsequent experimental publications in Phys. Rev. Lett. by Stander et al. [146] as well as in Nature Physics by Young et al. [147]. These papers model Klein tunneling using a transmission formula where the transmission probability depends on the value of the magnetic field. Specifically, the angle of perfect transmission is shifted from $\phi=0$ to a value $\phi=\phi_{*}$ that depends on the magnetic field $\left(T \sim \exp \left(-(\sin (\phi)-a)^{2}\right)\right.$ see sec. 7.3 for details). This formula is challenged significantly with the numerical data presented in sec. 7.3 (in fact that numeric data do not agree at all with the formula).

Not only that, but the most recent experimental study of Klein tunneling, which uses almost 8 years of extra experimental progress, changes the picture dramatically. In the Science publication by Chen et al. [43] the authors claim to measure Klein tunneling using a transmission formula that has no dependence whatsoever on the magnetic field! So, which case is true? Does the transmission probability depend on the magnetic field or not? It is certain that more study needs to be done before we reach a conclusive answer, and this chapter contributes by using the semiclassical approach of the Husimi function.

A second difference here is that one cannot straight-forwardly apply the Husimi function for an electron moving in magnetic field, due to energy considerations. One first needs to extend the Husimi function so that it respects well-established physical principles regarding the energy of the electron. In sec. 7.1 this is problem is discussed, as well as solved (to the best of my knowledge for the first time in the literature).

\subsection{Husimi function in magnetic fields}

The $Q$ function is given by eq. (2.6.4), $Q[\psi]\left(\mathbf{r}_{0}, \mathbf{k}_{0} ; \sigma\right)=\frac{1}{\pi}\left|\left\langle\psi \mid \mathcal{W}\left(\mathbf{r}_{0}, \mathbf{k}_{0}, \sigma\right)\right\rangle\right|^{2}$, and relies on the definition of a "wavepacket" (i.e. coherent state), given by

$$
\mathcal{W}\left(\mathbf{r}_{0}, \mathbf{k}_{0} ; \sigma\right) \sim \exp \left(-\frac{\left(\mathbf{r}-\mathbf{r}_{0}\right)^{2}}{4 \sigma^{2}}+i \mathbf{k}_{0} \cdot \mathbf{r}\right) .
$$

(2.6.1 revisited)

How should this expression be modified in the case of particles moving in magnetic fields?

\subsubsection{The naive transformation}

In the presence of magnetic fields, the Hamiltonian of the system is typically transformed under minimal coupling, by setting $\mathbf{k} \rightarrow \mathbf{k}-c_{q} \mathbf{A} / \hbar$ with $c_{q}$ the particle charge (signed quantity) and 
A the vector potential. Mason, Borunda and Heller have suggested in [94] that one should use the same transformation for the wavepacket, $\mathbf{k}_{0} \rightarrow \mathbf{k}_{0}-c_{q} \mathbf{A} / \hbar$.

To check the validity of the suggestion, consider the energy of the wavepacket before and after adding a magnetic field. We do not need to go into the case of graphene, using the Dirac Hamiltonian; for this illustration the standard Schrödinger Hamiltonian is enough. Under a vector potential A the Schrödinger Hamiltonian becomes

$$
H_{S, \mathbf{A}}=-\frac{\hbar^{2}}{2 m} \nabla^{2}+i \frac{c_{q} \hbar}{m} \mathbf{A} \cdot \nabla+\frac{c_{q}^{2}}{2 m} \mathbf{A}^{2}
$$

(using $\mathbf{k}=-i \nabla$ and being in absence of any scalar potentials). In following computations I set $\hbar=m=c_{q}=\sigma=1$ for simplicity. The energy of the wavepacket of eq. (2.6.1) with $H_{S, \mathbf{A}=0}$ is

$$
\left\langle\mathcal{W}\left|H_{S, 0}\right| \mathcal{W}\right\rangle=\iint \mathcal{W}^{*} H_{S, 0} \mathcal{W} d x d y=\frac{\pi}{4}\left(k_{0, x}^{2}+k_{0, y}^{2}+2\right) .
$$

To add the magnetic field, we modify $\mathcal{W}$ according to Mason et. al [94] as

$$
\mathcal{W}_{\text {Mason }}:=\mathcal{W} \exp \left(-i c_{q} \mathbf{A} \cdot \mathbf{r}\right)
$$

We calculate again eq. (7.1.2) using the Landau gauge with $\mathbf{A}_{L}=c_{q} B(-y, 0)$

$$
\left\langle\mathcal{W}_{\text {Mason }}\left|H_{S, \mathbf{A}}\right| \mathcal{W}_{\text {Mason }}\right\rangle=\frac{\pi}{16}\left(5 B^{2}+4\left(2 B y_{0}+k_{0, x}\right)^{2}+4\left(B x_{0}+k_{0, y}\right)^{2}+8\right) \text {. }
$$

This result is surprising, as it shows that the energy of this wavepacket depends on both $x_{0}$ and $y_{0}$ (similar results are obtained with any gauge). The immediate technical problem that this entails is that the wavepackets are initialized with different energy (thus, in different parts of the dispersion relation) for different initial positions. This is almost always not what one wants. But there is a bigger problem here. The expression is enforcing an arbitrary origin to the real space (where are $x_{0}, y_{0}$ measured from?), even though there are no scalar potentials to dictate it! This result is of course unphysical, and very difficult to make sense of, therefore, eq. (7.1.3) cannot be the correct way to proceed.

\subsubsection{Magnetic translation operator: the correct transformation}

The naive transformation of eq. (7.1.3) of the previous section cannot properly create wavepackets in magnetic fields. We have to find a different approach, in order to have the wavepacket manifest at any center $\mathbf{r}_{0}$ while respecting the energy independence from $\mathbf{r}_{0}$. It is very surprising that in the existing literature the Husimi function has never been properly used to study electrons moving in magnetic fields.

Fortunately, a similar problem has been considered before but in other context (tight-binding theory). It was solved in 1964 independently by Brown [148] and Zak [149], where each one invented the magnetic translation operator group. This operator group translates a wavefunction from one location to another one, while in the presence of magnetic fields. We follow the approach of Brown, which states that the translation operator in magnetic fields is expressed as

$$
\hat{T}_{M}(\mathbf{R})=\exp \left(-i \mathbf{R} \cdot\left(\mathbf{p}-c_{q} \mathbf{A}\right) / \hbar\right)=\exp \left(i c_{q} \mathbf{R} \cdot \mathbf{A} / \hbar\right) \hat{T}(\mathbf{R})
$$

where $\hat{T}(\mathbf{R})$ is the known translation operator, shifting a wavefunction from position $\mathbf{r}$ to $\mathbf{r}+\mathbf{R}$. It is important to take note that eq. (7.1.5) is valid only in the case of the symmetric gauge, i.e. $\mathbf{A}_{S}=-\frac{1}{2}(\mathbf{r} \times \mathbf{B})$.

The operator $\hat{T}_{M}\left(\mathbf{r}_{0}\right)$ must first be applied to the wavepacket of eq. (2.6.1) (setting its $\mathbf{r}_{0}=0$ first) to properly make the wavepacket centered at $\mathbf{r}_{0}$. Then, one more transformation must be made. Because the expression of eq. (7.1.5) is valid only in the symmetric gauge, while in 
the simulations presented in this thesis I operate in the Landau gauge, the wavepacket must be further manipulated to transform from the symmetric to the Landau gauge. This transformation is straight-forward: given a wavefunction $\psi$ in some gauge $\mathbf{A}_{S}$, one changes the gauge to $\mathbf{A}_{L}$ by multiplying the wavefunction with $\exp \left(i \frac{c_{q}}{\hbar} \Lambda_{S \rightarrow L}\right)$ with $\Lambda_{S \rightarrow L}$ the gauge transform. Assuming $\mathbf{B}=B \hat{z}$ the transformation from the symmetric to Landau gauge is

$$
\Lambda_{S \rightarrow L}=\left(-\frac{B}{2} x y\right), \quad \mathbf{A}_{L}=\mathbf{A}_{S}+\nabla \Lambda_{S \rightarrow L}
$$

for the Landau gauge oriented in the $x$ direction, $\mathbf{A}_{L}=-B y \hat{x}$.

Sequentially applying all the transformations we arrive at what I will call magnetic Gaussian wavepacket (in the Landau gauge and for 2 dimensional space)

$$
\begin{aligned}
\mathcal{M}_{L}\left(\mathbf{r}_{0}, \mathbf{k}_{0}, B ; \sigma\right) & =\exp \left(i \frac{c_{q}}{\hbar} \Lambda_{S \rightarrow L}\right) \exp \left(i \frac{c_{q}}{\hbar} \mathbf{r}_{0} \cdot \mathbf{A}_{S}\right) \hat{T}\left(\mathbf{r}_{0}\right) \mathcal{W}\left(0, \mathbf{k}_{0} ; \sigma\right) \\
& =N_{\sigma} \exp \left(-\frac{\left(\mathbf{r}-\mathbf{r}_{0}\right)^{2}}{4 \sigma^{2}}+i \mathbf{k}_{0} \cdot\left(\mathbf{r}-\mathbf{r}_{0}\right)\right) \exp \left(-i \frac{c_{q}}{\hbar} \frac{B}{2}\left(x y-x_{0} y+x y_{0}\right)\right)
\end{aligned}
$$

What we see is that the phase factors of eq. (7.1.7) are not trivially related to the gauge, like for example simply $-c_{q} \mathbf{A}_{L} / \hbar$, as was suggested in the preceding section.

Let us now revisit the integral of sec. 7.1.1. The energy of this new magnetic wavepacket in a 2D continuous space with the Schrödinger Hamiltonian yields (again setting $\hbar=m=\sigma=$ $\left.c_{q}=1\right)$

$$
\left\langle H_{S, \mathbf{A}=\mathbf{A}_{L}}\left|\mathcal{M}_{L}\right| H_{S, \mathbf{A}=\mathbf{A}_{L}}\right\rangle=\frac{\pi}{32}\left(B^{2}+8\left(k_{0, x}^{2}+k_{0, y}^{2}+2\right)\right)
$$

which has no dependence on $x_{0}, y_{0}$ as it should. Notice that the energy does depend on the magnetic field, and this is an expected result. It stems from the last term of eq. (7.1.1), which is known as the diamagnetic term. Normally this contribution is small, as it is multiplied by a factor of $c_{q}^{2} / m$.

\subsubsection{Magnetic wavepacket energy in a tight-binding system}

To test the validity of the magnetic wavepacket also in discretized space (i.e. crystalline lattice), I use a trivial graphene rectangle, as shown in Fig. 7.1. What we want to compare is the energy of the wavepacket when its center is moved around the lattice. Physically, this energy should not depend on the location, because the lattice is in the absence of scalar potentials, and $\sigma$ is small enough that finite-size effects should not matter.

Setting up the tight-binding system is identical like in the third paper of this thesis (chapter 6). The only change is that now there is also an magnetic field. The details of how to apply a magnetic field in a tight-binding system are discussed in the appendix at the end of the current chapter. Then different wavepackets are created in the lattice, with eqs. (7.1.3), (7.1.7), as well as the unmodified version eq. (2.6.1), while the Hamiltonian matrix $H$ is obtained straightforwardly from Kwant. $H$ here is just a sparse matrix whose entries give either the hopping amplitudes or the onsite energies. The product $\psi^{*} H \psi$ thus gives the energy of the wavepacket. The table of Fig. 7.1 compares the differences

$$
\left|\psi\left(\mathbf{r}_{j}\right)^{*} H \psi\left(\mathbf{r}_{j}\right)-\psi\left(\mathbf{r}_{i}\right)^{*} H \psi\left(\mathbf{r}_{i}\right)\right|
$$

for $i=1$ and $j=2,3,4$, and the locations $\mathbf{r}$ are highlighted in the figure.

The Hamiltonian matrix is the same across all cases, using the standard Landau gauge (see appendix). In the table only the energy difference across different positions is shown, because no care was taken to choose proper units or to normalize the wavepackets. We first see the expected result, i.e. that the energy of a unmodified wavepacket is not independent of its position. We 


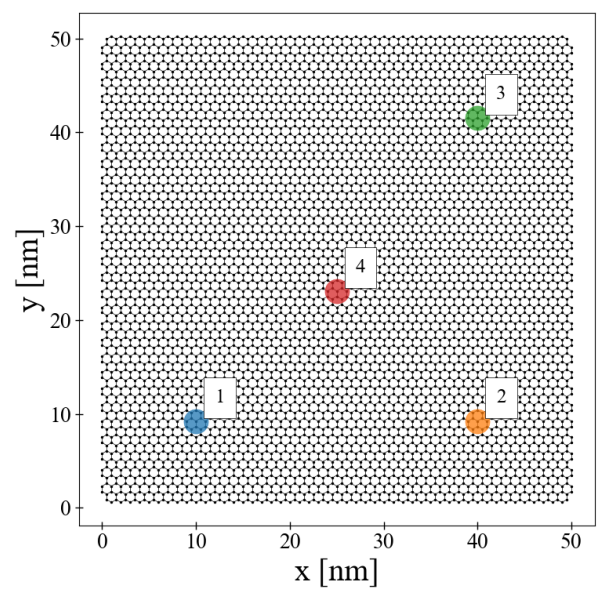

Energy difference between wavepackets

\begin{tabular}{c|ccc}
\hline Positions & Standard & Mason & Magnetic \\
\hline$(2,1)$ & $3.554 \mathrm{E}-15$ & $4.715 \mathrm{E}-01$ & $1.776 \mathrm{E}-15$ \\
$(3,1)$ & $1.011 \mathrm{E}-01$ & $5.724 \mathrm{E}-01$ & $1.776 \mathrm{E}-15$ \\
$(4,1)$ & $5.158 \mathrm{E}-02$ & $3.181 \mathrm{E}-01$ & $2.664 \mathrm{E}-15$ \\
\hline
\end{tabular}

Figure 7.1: Energy difference between wavepackets centered at different positions, see the first column of the table as well as the graphic. For the locations 1, 2 and 3 the wavepacket centers have exactly the same distances from the edges of the sample. The formula used for the wavepacket is noted with the titles "Standard", "Mason" or "Magnetic". Standard uses eq. (2.6.1), Mason uses eq. (7.1.3) while Magnetic uses eq. (7.1.7). Notice that the energy differences in the magnetic case equals the machine precision.

also see that the same holds true for the modification suggested by Mason et al. Finally, we can see that the magnetic wavepacket defined in this chapter does indeed have energy independent of its position (within numerical accuracy).

\subsection{Application to a graphene nanodevice}

This section applies the Husimi function in magnetic fields, by using the magnetic wavepacket defined in the preceding section. This application is done in scattering wavefunctions of the device A, which is described in detail in the third paper of this dissertation, chapter 6 . The difference here is of course that there is an additional external (and constant) magnetic field. Similarly with chapter 6 , the Husimi function $Q$ is calculated at a given energy contour by scanning it at different wavevector angles $\phi$. Notice that the energy value is used here is the incoming energy $E$. It should be said however that this is slightly inaccurate as the energy is increasing a bit by adding a magnetic field. Because the increase is slight, and also because at the moment the discussion here focuses on angle resolution (which would be the same even if the Husimi function was localized at a contour of higher energy) this slight inaccuracy is insignificant.

Example Husimi functions of device A are shown in Fig. 7.2. One can immediately "see" the magnetic field just by looking at $Q(\phi, y)$. In the $B=0$ the expected result is seen, and $Q(y)$ localizes into two maxima, since for $m=2$ by definition the wavefunction has one node (besides the extra two at the device edge). For $B>0$ we first see that the incoming angle drastically changes, including its distribution. In addition $Q(y)$ is no longer spread out throughout the width of the device. Instead it becomes localized at large $y$, which correspond to the upper part of the waveguide, which is exactly what one should expect: the incoming modes in this case can be perceived as edge states (Landau levels) or skipping orbits that hop along the edge of the device. It therefore makes sense for $Q(y)$ to be localized mostly in the top of the device (notice that for positive $B$ we have counterclockwise rotation). Before the pn-junction and by summing over all valleys $Q$ is still an even function of $\phi$. This comes from the the pre-existing translational symmetry over $x$, which still exists even though there is an applied magnetic field.

The Husimi function is also very useful for obtaining a "particle" (i.e. localized wavepacket) to represent the scattered wavefunction. To do this let us will focus on the region after the pn 
Device A: $E_{F}=0, L=106, V_{0}=0.4, W=80, w=10$
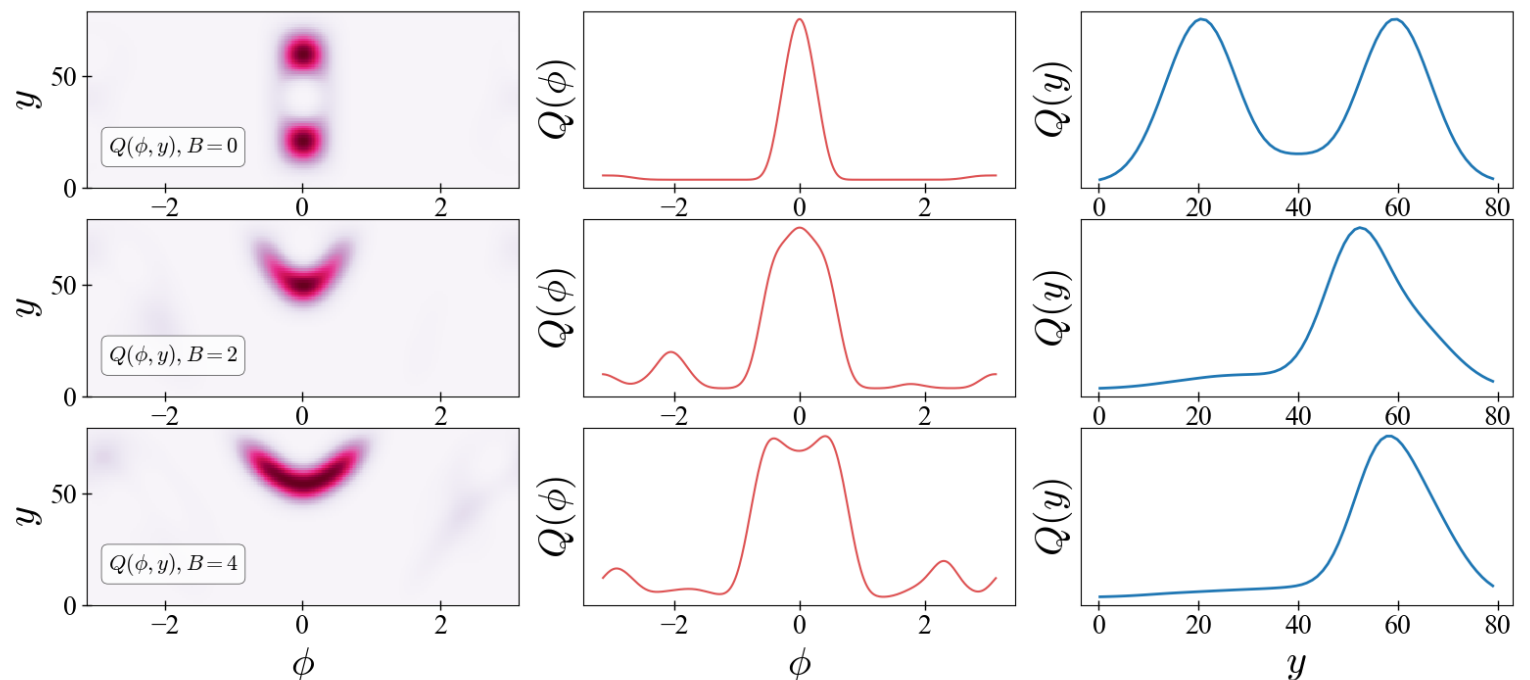

Figure 7.2: Husimi functions of the second incoming mode of device A for various magnetic fields. First column is the Husimi function, while second and third columns are the marginal distributions of $\phi$ and $y$ respectively. Here $Q$ is measured at a distance $3 \sigma$ before the pn-junction, with $\sigma=8$.

junction, as shown in Fig. 7.3, to avoid the interference. There $Q$ is calculated in a given $x_{0}$ slice, as described in chapter 6 and using the magnetic wavepacket of sec. 7.1. The maximum of $Q$ is then used to obtain an initial position $y_{0}$ and angle $\phi_{0}$. The initial conditions $\left(x_{0}, y_{0}, \phi_{0}\right)$ are then further used to a particle in a standard rectangular billiard (with same size and shape as the device) and applied magnetic field $-B$ (because in the hole regime, which is after the pn-junction, the felt field effectively changes sign), using DynamicalBilliards.jl, see chapter 4.

It is astonishing how well the particle trajectory fits on top of the wavefunction, establishing not only the validity of $Q$ but also its accuracy in the case of applied magnetic fields. Notice that this accuracy does not depend on the specific $x$ location that I measure $Q$ at; this was not hand picked, and to prove this I supplement my thesis with a video file that shows the plot of Fig. 7.3 as an animation scanned over several $x$ slices.

\subsection{Klein tunneling in a pn-junction with magnetic field}

In Ref. [145] and the follow-up work the transmission probability to tunnel through a pn-junction is modified with a magnetic field. In its most general form it can be written as [144, 145, 147, 146] (see e.g. equation (10) of Ref. [144])

$$
T(\phi)=\exp \left(-\gamma\left(\sin \phi-B / B^{*}\right)^{2}\right)
$$

with $\gamma$ a parameter that typically depends on the pn junction functional form as well as its parameters (e.g. the width of the junction) and also the incoming energy, and $B^{*}$ a field normalization that depends on the same (or similar) parameters. Details of these parameters do not matter, as in this section only qualitative comparisons will be made with eq. (7.3.1) (but as an example, for the setup of device A with a junction width of $10 \mathrm{~nm}$ one has $B^{*} \approx 40$ Tesla).

What this formula states is that as the strength of the applied field is increased there is still an angle $\phi_{*}=\arcsin \left(B / B_{*}\right)$ where the transmission remains perfect, in the spirit of Klein tunneling. This angle $\phi_{*}$ non-zero and is increased as $B$ is increased (up to a critical value). In addition, there is still a collimation effect around $\phi_{*}$. Conceptual issues arise in this approach. One might attempt to interpret eq. (7.3.1) by claiming that the term $B / B_{*}$ comes from the 


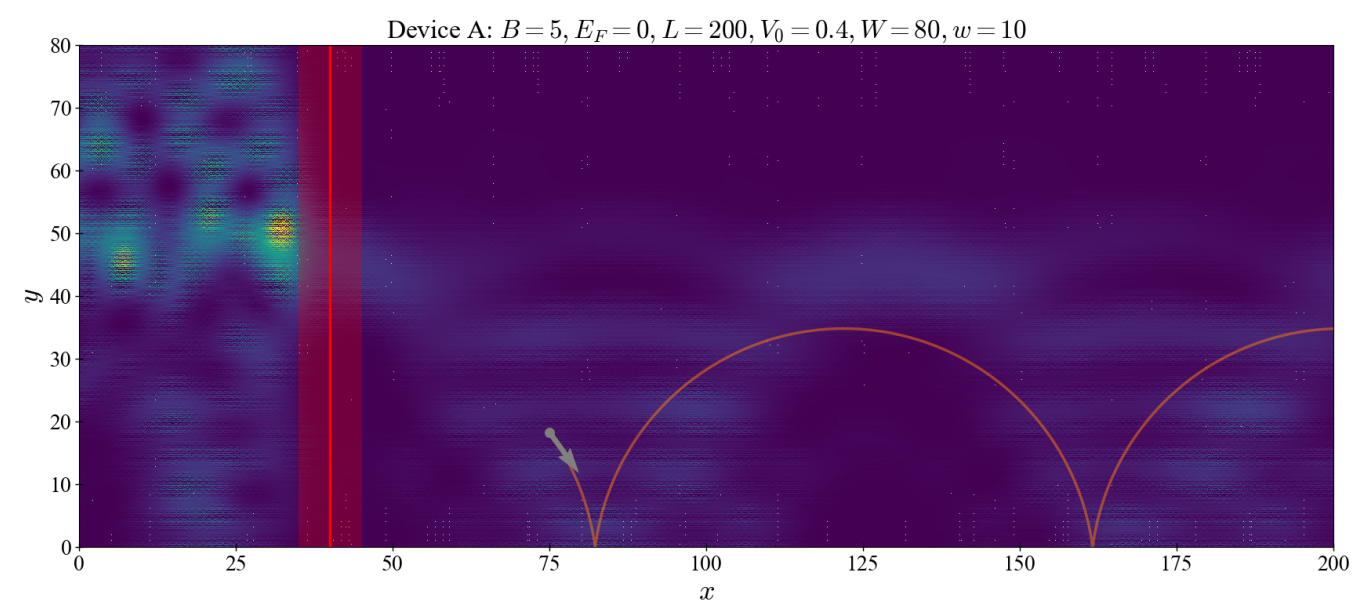

Figure 7.3: A scattering wavefunction inside device A. The red rectangle denotes the pn-junction, while the colormap denotes the wavefunction amplitude (yellow means highest) in arbitrary units. On top of the wavefunction a particle is plotted which is simulated with DynamicalBilliards.jl, see chapter 4. The initial conditions for the particle, noted by a gray dot and arrow, are obtained through the Husimi function at a specific $x$ slice. The orbit of the particle is plotted in orange color.

deflection of the electron ray from its starting angle until reaching the junction. This is false, however, since in no point in Refs. $[144,145,147,146]$ this is discussed and in addition in eq. (7.3.1) the distance from injection point to junction is completely absent as a parameter.

Eq. (7.3.1) is also in direct opposition with the latest experimental paper of Chen et al. [43] where the transmission probability does not depend on the magnetic field. The following two subsections present two different numeric facts that I believe also do not align with eq. (7.3.1). The first does not depend in any way on the Husimi function while the second utilizes the magnetic wavepackets of sec. 7.1.

\subsubsection{Transmission versus mode number}

In this subsection, the transmission of each mode $T_{m}$ versus the mode number $m$ is presented. Similarly with the previous sections, device A is simulated, with an applied perpendicular magnetic field of value $B$ (in Tesla). Various measurements of $T_{m}$ vs. $m$ for different magnetic field values are shown in Fig. 7.4.

There is one thing that deserves particular focus in this figure: as the magnetic field is increased, there is no longer any mode near perfect transmission. This appears to be in contradiction with eq. (7.3.1), according to which some mode should have perfect (or at least near perfect) transmission. One might argue that it could be that none of the modes has an angle that is in the perfect sweet spot of $\phi_{*}$. Even though this may seem valid, we already know from ch. 6 that increasing the mode number increases the incoming angle of incidence and thus we have a decent scan of the possible incoming angles that would allow us to find at least one mode with incoming angle near $\phi_{*}$. In addition, looking at high magnetic fields, e.g. $B=5-6 \mathrm{~T}$ we see that most modes almost the same transmission value: not only isn't there a mode with perfect transmission, but also all modes have significantly non-zero transmission, which once 


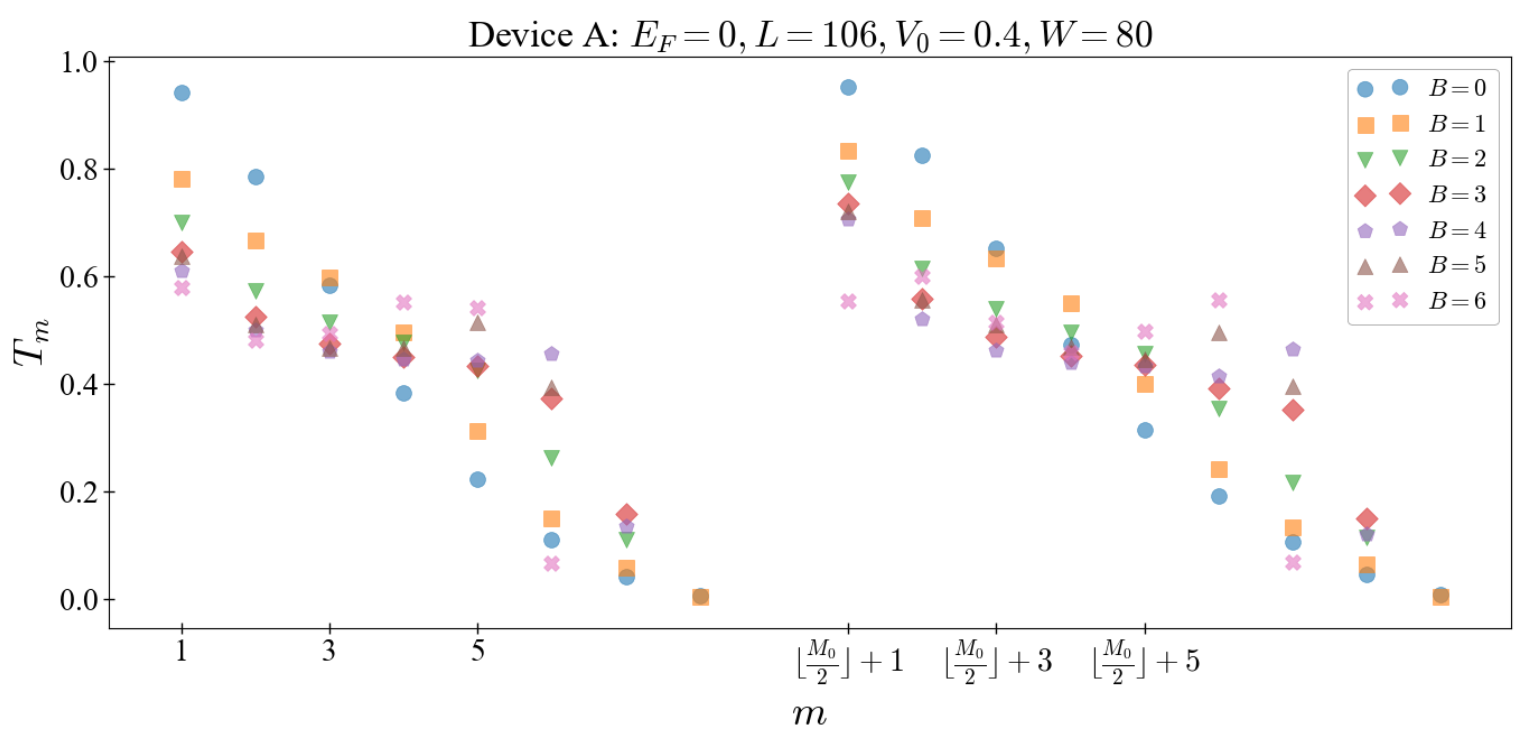

Figure 7.4: Mode transmission $T_{m}$ versus mode number $m$ for different magnetic fields in device A. Because as the magnetic field strength is increased some available open channels may close, the modes are plotted based on the two Dirac valleys discussed in the third paper of chapter 6 (all modes start from 1 to $M \div 2$ and then again from $M \div 2+1$ to their end). The magnetic field is stated in Tesla.

again does not agree with eq. (7.3.1) and its collimation effect.

\subsubsection{Transmission versus incoming angle}

Here the Husimi function is used to get a representation of an "incoming angle" for each scattering wavefunction in device A with magnetic field. This process is identical with the process followed in chapter 6 , keeping in mind the energy caveat discussed in sec. 7.2. Summing $Q$ over all valleys, integrating to obtain $Q(\phi)$ and then using the $\operatorname{argmax}$ of $Q(\phi)$ gives an estimate of an "incoming angle", $\Phi_{m}$, which is preserved all the way up to the pn-junction, due to the translational symmetry over $x$.

In Fig. 7.5 presents the results, which show some interesting behavior. As the magnetic field strength is increased a "thermalization" process seems to occur. All incoming modes tend to have equal transmission value, even if the incoming angle is clearly different. This fact does not agree with eq. (7.3.1) which suggests an exponential decrease of $T$ shifted to some different origin other than $\phi=0$.

\section{Appendix: Peierls Substitution}

To apply a magnetic field in a tight binding system one modifies the hopping elements $t_{m n}$ according to [8]

$$
t_{m n} \rightarrow t_{m n} e^{i \phi_{m n}} ; \quad \phi_{m n}=\frac{c_{q}}{\hbar} \int_{\mathbf{R}_{n}}^{\mathbf{R}_{m}} \mathbf{A} \cdot d \mathbf{r}
$$

with $c_{q}$ the charge of the particle. The integral is from $n$ to $m$ because the hopping element $t_{m n}$ corresponds to going from $n$ to $m$ (so the second index is the source and the first index is the target). In the case of uniform magnetic fields the expression simplifies to

$$
\phi_{\mathbf{m n}}=\frac{c_{q}}{\hbar} \mathbf{A}\left(\frac{\mathbf{r}_{\mathbf{m}}+\mathbf{r}_{\mathbf{n}}}{2}\right) \cdot\left(\mathbf{r}_{\mathbf{m}}-\mathbf{r}_{\mathbf{n}}\right) .
$$

Notice that in the last equation the vector potential's value is evaluated at the mid-point of the two lattice sites. 

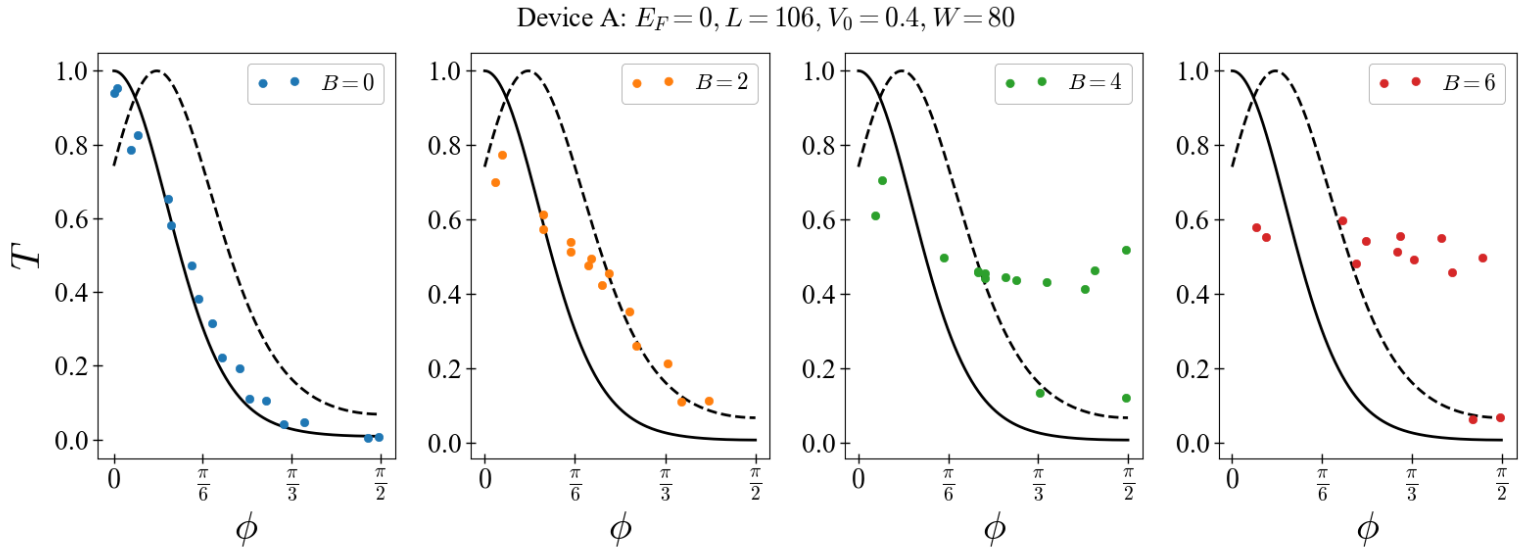

Figure 7.5: Transmission of each incoming mode versus angle of incidence for device $A$ in various magnetic fields. The scatter points are $T_{m} v s . \Phi_{m}$, the angle deduced using the Husimi function. The solid black curves are the transmission probability for no magnetic field, eq. (2.3.26) for the parameters of the device. The dashed curved is eq. (7.3.1) for $\gamma=\pi k_{F} w / 2$ (i.e. same prefactor as the non-magnetic case) and $B / B^{*}=0.25$ (arbitrary choice for qualitative comparison).

The common case of a 2D material with a perpendicular magnetic field is the constant one, $\mathbf{B}=B \hat{z}$. Since it must hold that $\mathbf{B}=\nabla \times \mathbf{A}$, one has the possibility to choose one of the many available gauges for the vector potential. A good approach is to choose a gauge that is independent of the invariant direction of a lead. For example, the leads of the system are along the direction of the $\hat{x}$ axis, then we can choose $\mathbf{A}=-B y \hat{x}$ which gives the expected $\mathbf{B}$. In this case, the integral gives

$$
\phi_{m n}=-\frac{c_{q}}{\hbar} B \frac{y_{n}+y_{m}}{2}\left(x_{m}-x_{n}\right)
$$

for the case where the two sites do not share the same $y$ coordinate, such as the case of nearest neighbors in graphene. Notice that the coordinates are in real-space, not lattice space. Equivalently for the case of translational invariance along $\hat{y}$, one would use $\mathbf{A}=B x \hat{y}$ and obtain

$$
\phi_{m n}=\frac{c_{q}}{\hbar} B \frac{x_{n}+x_{m}}{2}\left(y_{m}-y_{n}\right) .
$$




\section{Summary \& Outlook}

\subsection{Summary of results}

In my thesis I studied electron dynamics in nanodevices and billiards. Of course, billiards themselves are often used as model systems for real devices. My work revolved around connecting transport properties and quantum effects with the phase space of the electron dynamics. On one hand, this was done directly by studying classical dynamics using the theory of dynamical systems and billiards. On the other hard, I simulated quantum systems and then studied them through the semiclassical lens of the Husimi function.

The first project of my dissertation, chapter 3, studied the electron movement in antidot superlattices by modeling the system using the single electron picture and classical (Hamiltonian) mechanics. The main result of this work was showing that the resistance of the antidot superlattice can be connected with the chaotic phase space volume of the system's dynamics. We used our findings to explain exactly the mechanism behind classical features of the magnetoresistance, like the commensurability peaks. In doing so, we also resolved a long-standing controversy about which part of the dynamics leads to the resistance peaks. What happens in the end is that the mere existence of periodic orbits in the phase space reduce the mean collision time of the chaotic electrons, thus leading to peaks in the magnetoresistance. During this project I also developed and published a high quality software for simulating dynamical billiards, showcased in chapter 4. This software was used and extended by other members of the scientific community as well [141].

Explaining the mechanism behind the commensurability peaks involved an analytic proof of the connection between phase space volumes and characteristic timescales in the antidot superlattice. This proof used an extremely general and simple theorem called Kac's lemma. This lemma is a proportionality relation that holds true for all measure-preserving maps (which all of Hamiltonian mechanics are), and states that mean recurrence times in the phase space are proportional to phase space volumes. The second project of my thesis, chapter 5 , applied this general lemma to study billiards, and more specifically their Lyapunov exponent, in detail. The project led to several results. Firstly, we provided an analytic expression for the Lyapunov exponent of the periodic Sinai billiard. Secondly, we showed that in very diverse billiards, the Lyapunov exponent has a leading contribution inverse with the chaotic phase space volume of the billiard. We also provided strong arguments on why this should happen for all billiards, not just the ones considered in the paper. Thirdly, we formulated a conceptual model that allows one to estimate the Lyapunov exponent and connect it with the parameters of the billiard.

In the second half of my thesis I studied quantum systems, but even so once again the goal was to connect aspects of the system with the phase space. This was achieved by using the Husimi function, a tool that transforms a wavefunction into a phase space distribution. I used this tool to study in detail quantum phenomena specific to graphene, by simulating simple, but paradigmatic, graphene nanodevices. Chapter 6 starts by establishing the Husimi function as an accurate tool to study transport in nanodevices, and even in relatively small tightbinding systems. It then proceeds to display several findings, some surprisingly missing from the literature even though graphene is such a well studied material. For example, in our paper 
we showed that a pn-junction has significant asymmetry in its intervalley scattering process, effectively scattering only one of the two valleys. We explain this based on the form of the dispersion relation of a graphene nanoribbon. To properly study intervalley scattering, we also provided an intuitive and quantitative measure for intervalley scattering, that can be applied generically in other materials besides graphene. Another important result was a quantitative assessment of intervalley scattering from graphene edges, showing that armchair edges are the strongest intervalley scatterers present in graphene (which was suggested several times in the literature). Coming back to Klein tunneling, we showed that it is possible to use the Husimi function as a probability distribution to uncover the transmission probabilities of each incoming mode (assuming knowledge of Klein tunneling), and we further used this to confirm that Klein tunneling takes place even in much more complicated geometries. Lastly, we did an analysis on the nature of Klein tunneling for higher energies, which to the best of my knowledge has never been done before in the literature. Besides these graphene-specific results, I believe that this manuscript provides a strong case of why the Husimi function should be more generally adopted by the condensed matter community and become a standard tool of the field.

In the last project of my thesis, chapter 7, I continued the approach of using the Husimi functions but for propagation in magnetic fields. To be able to apply the Husimi function in this context, I first had to extend it to magnetic fields, so that energy considerations are respected. Numeric analysis confirmed the validity of my extension and also showed that it is possible to obtain "particles" (localized wavepackets) in nanodevices using the Husmi function. Then, Klein tunneling was examined in detail, and was compared with standing theories in the literature. Several numeric findings were presented, some also using the newly extended Husimi function, that seem to contradict the standing theory. These numeric results show that Klein tunneling in magnetic fields (in real, finitely-sized nanodevices) is not yet perfectly understood and more analysis is necessary.

\subsection{Outlook}

In this final section of my dissertation it is fitting to discuss the impact and potential application of the work and results that compose the thesis. The first project about the mechanisms of antidot superlattices can be interpreted as a case study. The main implications, that could have broad impact in other scenarios, can be formulated as follows. Transport timescales in nanodevices seem to be of crucial importance, and it is possible to connect them with macroscopic observables. This was shown to be true in detail for the antidot superlattices, but another example system would be the average transit time through a quantum dot and its connection to the conductivity. In addition, a working assumption is that dynamical features with timescales larger than the mean free time will be inaccessible by experiments. For the case of the antidot superlattices this seems to be confirmed by experiments, as in the measurements one cannot see neither commensurability peaks (chapter 3) nor the negative Hall effect (sec. 3.1) for mean free times smaller than $\kappa$, the mean collision time. Therefore experimentalists should be aware of the timescales of the dynamics that they are trying to measure before they perform their measurements, as they first need to compare the mean free time with the appropriate timescale. Besides this, since we also showed that it is possible to connect a characteristic timescale in an antidot superlattice with the corresponding phase space volume. Due to the very general ingredients leading to such connection (essentially volume conservation) one could see this being possible in may other Hamiltonian systems, relevant for other experiments or theoretical studies not considered here. Notice that this also has further impact: sometimes phase space restrictions dictate exactly how the phase space should change with respect to a change in an external parameter. In our case this could directly explain why the chaotic phase space volume is decreased when the magnetic field is changed, and such an explanation could be carried out in different scenarios as well. 
The second project showed that the leading contribution to the Lyapunov exponent in billiards is inversely proportional with the chaotic phase space volume. This finding by itself is of broad interest from the theoretical perspective, since very little was known about the Lyapunov exponent of billiards before we completed our work. But besides this theoretical impact, there is also a practical one. In our work we formulated a conceptual model that allows one to estimate the Lyapunov exponent and connect it with the parameters of the billiard. This is relevant for all the fields that use billiards to model real world systems. When one wants to study real physical systems that can be approximated by billiards, the role of the Lyapunov exponent is indirect. For example, it sets a timescale which governs correlation decays (and thus conductivities in experimental systems) or echo signals. To understand, and not just numerically reproduce, the parameter dependence of such experimental signals (for example the magnetic field dependence of the conductivity) it can be extremely valuable to know at least approximately the connection between the Lyapunov exponent and the parameters of the system.

One of the goals of chapter 6 was to prove that the Husimi function is a tool that brings benefits to the study of nanodevices from a semiclassical perspective. Because in this chapter I presented several findings that have either not been discussed at all in the literature, or have been discussed only qualitatively, I believe a strong case is being made for wider adoption of the Husimi function. In addition, some of the numeric findings presented definitely deserve further study, for example the connection between the group velocity and the wavevector angle with the transmission probability at higher energies. (To the best of my knowledge) neither theory nor experiments have been performed in a sufficient amount in this regime but the numeric results of section II.D and II.E of chapter 6 seem to be interesting, if not immediately from an application perspective, definitely from a theory perspective. Besides being a tool to measure quantities, using the marginal distributions obtained from the Husimi one can even calculate the transmission probability through e.g. a pn-junction, using theoretical formulas. This is yet one more reason to use the Husimi function in condensed matter.

Chapter 7, which discusses the Husimi function and Klein tunneling in magnetic fields, provides both new tools as well as open questions. The extension of $Q$ to magnetic fields provides a new tool for studying transport from the semiclassical perspective in the presence of magnetic fields. This new tool can be used to study both the open questions of chapter 7 as well as questions in other context. It also remains to be seen what new insights can the magnetic wavepackets of chapter 7 provide for other scenarios, besides Klein tunneling. For example, one case to study is magnetic focusing, which is an effect that has been studied extensively from different perspectives. Since the effect relies heavily on the concept of the cyclotron radius, semiclassical approaches are naturally fitting to analyze it from new perspectives. This will also be yet one more case study to strengthen adoption of the Husimi function by the condensed matter community.

The big open question of chapter 7 is what happens with Klein tunneling in magnetic fields. The numerical results of the chapter show that the effect is not well understood yet, since the results do not align with the existing theoretical predictions of the effect (and the same can be said for recent experiments). Therefore, more steps have to be taken in understanding it better. One approach, already partly implemented in this thesis, is to use the Husimi function in magnetic fields. Other approaches are also possible, for example wavepacket dynamics, localized electron beams in larger systems (as in [150]) or ray-splitting billiards. It is important to use these tools to understand not only why eq. (7.3.1) does not work in the context studied in this thesis, but also to find out the correct answer and explain the physics of Klein tunneling in magnetic fields. 


\section{Acknowledgments}

First and foremost I want to thank everyone I had the unique fortune of being guided by. Besides having the obvious invaluable input for my thesis, my two supervisors in this endeavor, Ragnar Fleischmann and Theo Geisel, have sharpened and polished my scientific skills and certainly made me a much better person than when I arrived in Göttingen. The remaining two members of the the thesis Advisory Committee, Michael Wilczek and Stephan Herminghaus, also gave me a tremendous amount of support, both scientific and emotional, helping me go through the infamous "hard phases of a PhD". Ulrich Parlitz, another person I was blessed to meet, gave me invaluable support during the later stages of my career, e.g. when preparing for Postdoctoral positions. In a similar spirit, I am in debt to my colleagues and friends that read my thesis and helped in greatly in improving it: Gerrit, Martin, Joao and Phillip. It should also go without saying, that I owe a big thank you to Jonathan Eroms and Andreas Sandner, who performed the experiments on antidot superlattices that initialized my work, had numerous fruitful discussions with me about the physics of graphene, as well as shared with me the new devices that kickstarted the Husimi function project.

I also want to thank the people I worked with in many fascinating scientific projects, some included in this thesis, while the others unfortunately not. Lukas Hupe was an excellent Bachelor student I had the pleasure of supervising. He did with me and Ragnar Fleischmann the project about the Lyapunov exponents of billiards that composed ch. 5. In addition Lukas had major contributions to the software DynamicalBilliards.jl. Jonas Isensee is yet another excellent Bachelor student that did his thesis with me, co-supervised with Ulrich Parlitz. With Jonas I had the pleasure to work on timeseries prediction. Last in this list of amazing collaborators are Annika Ziereis, Thorsten Albrecht, York Hagmayer and Viola Priesemann with whom I worked with in a project conceptualized by Theo Geisel. It was a tremendous pleasure working with them on the project that made me apply to Max Planck Institute for Dynamics and Self-Organization in the first place: analysis and manipulation of music timeseries. I am grateful to have worked with such a diverse group of people, not only due to the interesting projects that they offered, but also because all of them having a different expertise gave me unique tools for the rest of my career.

Lastly I wish to thank friends and family. All the students of the Max Planck Research Groups in the institute and more specifically JD, Jason, Mirna, Philipp, Joao, Gerrit and Joao. My friends from my University days, Bill, Dora, Tasos, Thodo and George. My absolutely amazing band, the Max Funk Institute: Darrin, Lucas, Alfred, Tillman, Chahira, Marek and Martijn. My family back in Greece, composed of too many members to name, yet all of them care for me wholeheartedly. And finally my mother, Angeliki, to whom I owe most of the things I have achieved. 


\section{Bibliography}

[1] Wikipedia contributors, "Information age - Wikipedia, the free encyclopedia," 2019. [Online; accessed 13-July-2019].

[2] A. Narayanan, J. Bonneau, E. Felten, A. Miller, and S. Goldfeder, Bitcoin and Cryptocurrency Technologies: A Comprehensive Introduction. Princeton University Press, 2016.

[3] J. Liang, L. Li, and D. Zeng, "Evolutionary dynamics of cryptocurrency transaction networks: An empirical study," PLOS ONE, vol. 13, p. e0202202, Aug. 2018.

[4] R. B. Alley, K. A. Emanuel, and F. Zhang, "Advances in weather prediction," Science, vol. 363, pp. 342-344, Jan. 2019.

[5] T. Mauritsen, J. Bader, T. Becker, J. Behrens, M. Bittner, R. Brokopf, V. Brovkin, M. Claussen, T. Crueger, M. Esch, I. Fast, S. Fiedler, D. Fläschner, V. Gayler, M. Giorgetta, D. S. Goll, H. Haak, S. Hagemann, C. Hedemann, C. Hohenegger, T. Ilyina, T. Jahns, D. J. de-la Cuesta, J. Jungclaus, T. Kleinen, S. Kloster, D. Kracher, S. Kinne, D. Kleberg, G. Lasslop, L. Kornblueh, J. Marotzke, D. Matei, K. Meraner, U. Mikolajewicz, K. Modali, B. Möbis, W. A. Müller, J. E. M. S. Nabel, C. C. W. Nam, D. Notz, S.-S. Nyawira, H. Paulsen, K. Peters, R. Pincus, H. Pohlmann, J. Pongratz, M. Popp, T. J. Raddatz, S. Rast, R. Redler, C. H. Reick, T. Rohrschneider, V. Schemann, H. Schmidt, R. Schnur, U. Schulzweida, K. D. Six, L. Stein, I. Stemmler, B. Stevens, J.-S. Storch, F. Tian, A. Voigt, P. Vrese, K.-H. Wieners, S. Wilkenskjeld, A. Winkler, and E. Roeckner, "Developments in the MPI-m earth system model version 1.2 (MPI-ESM1.2) and its response to increasing CO 2," Journal of Advances in Modeling Earth Systems, vol. 11, pp. 998-1038, Apr. 2019.

[6] A. D. King, J. Carrasquilla, J. Raymond, I. Ozfidan, E. Andriyash, A. Berkley, M. Reis, T. Lanting, R. Harris, F. Altomare, K. Boothby, P. I. Bunyk, C. Enderud, A. Fréchette, E. Hoskinson, N. Ladizinsky, T. Oh, G. Poulin-Lamarre, C. Rich, Y. Sato, A. Y. Smirnov, L. J. Swenson, M. H. Volkmann, J. Whittaker, J. Yao, E. Ladizinsky, M. W. Johnson, J. Hilton, and M. H. Amin, "Observation of topological phenomena in a programmable lattice of 1, 800 qubits," Nature, vol. 560, pp. 456-460, Aug. 2018.

[7] I. Ross, "The invention of the transistor," Proceedings of the IEEE, vol. 86, no. 1, pp. 7-28, 1998.

[8] S. Datta, Electronic Transport in Mesoscopic Systems. Cambridge University press, 1995.

[9] J. L. C. Daams, J. R. Rodgers, and P. Villars, "Typical interatomic distances: metals and alloys," in International Tables for Crystallography, pp. 774-777, International Union of Crystallography, Oct. 2006.

[10] S. J. M. . A. Ledwith, Chemistry of the Semiconductor Industry. Springer, 1989. 
[11] B. K. Teo and X. H. Sun, "Silicon-based low-dimensional nanomaterials and nanodevices," Chemical Reviews, vol. 107, pp. 1454-1532, May 2007.

[12] K. S. Novoselov, A. K. Geim, S. V. Morozov, D. Jiang, Y. Zhang, S. V. Dubonos, I. V. Grigorieva, and A. A. Firsov, "Electric field effect in atomically thin carbon films," Science (New York, N.Y.), vol. 306, no. 5696, pp. 666-669, 2004.

[13] K. S. Novoselov, A. K. Geim, S. V. Morozov, D. Jiang, M. I. Katsnelson, I. V. Grigorieva, S. V. Dubonos, and A. A. Firsov, "Two-dimensional gas of massless Dirac fermions in graphene," Nature, vol. 438, no. 7065, pp. 197-200, 2005.

[14] Y. Zhang, Y. W. Tan, H. L. Stormer, and P. Kim, "Experimental observation of the quantum Hall effect and Berry's phase in graphene," Nature, vol. 438, no. 7065, pp. 201-4, 2005.

[15] A. Geim and K. Novoselov, "The rise of graphene.," Nat. Mater., pp. 183-191, 2007.

[16] P. A. M. Dirac, "The quantum theory of the electron," Proceedings of the Royal Society A: Mathematical, Physical and Engineering Sciences, vol. 117, pp. 610-624, Feb. 1928.

[17] F. V. Kusmartsev, W. M. Wu, M. P. Pierpoint, and K. C. Yung, "Application of graphene within optoelectronic devices and transistors," 2014.

[18] A. A. Balandin, S. Ghosh, W. Bao, I. Calizo, D. Teweldebrhan, F. Miao, and C. N. Lau, "Superior thermal conductivity of single-layer graphene," Nano Letters, vol. 8, pp. 902-907, Mar. 2008.

[19] C. Lee, X. Wei, J. W. Kysar, and J. Hone, "Measurement of the elastic properties and intrinsic strength of monolayer graphene," Science, vol. 321, pp. 385-388, July 2008.

[20] a. H. Castro Neto, F. Guinea, N. M. R. Peres, K. S. Novoselov, and a. K. Geim, "The electronic properties of graphene," Reviews of Modern Physics, vol. 81, no. 1, pp. 109-162, 2009 .

[21] Wikipedia contributors, "Graphene - Wikipedia, the free encyclopedia," 2019. [Online; accessed 13-July-2019].

[22] Y. Li, H. Yuan, A. von dem Bussche, M. Creighton, R. H. Hurt, A. B. Kane, and H. Gao, "Graphene microsheets enter cells through spontaneous membrane penetration at edge asperities and corner sites," Proceedings of the National Academy of Sciences, vol. 110, pp. 12295-12300, July 2013.

[23] University of Manchester, "Graphene - applications," 2019. [Online; accessed 13-July-2019].

[24] Ron Mertens and Roni Peleg, "Graphene applications: what is graphene used for?," 2019. [Online; accessed 13-July-2019].

[25] W. Choi, I. Lahiri, R. Seelaboyina, and Y. S. Kang, "Synthesis of graphene and its applications: A review," Critical Reviews in Solid State and Materials Sciences, vol. 35, pp. 52-71, Feb. 2010.

[26] Wikipedia contributors, "Ballistic conduction - Wikipedia, the free encyclopedia," 2019. [Online; accessed 13-July-2019].

[27] N. W. Ashcroft and N. D. Mermin, Solid State Physics. Holt-Saunders, 1976.

[28] R. O. Jones, "Density functional theory: Its origins, rise to prominence, and future," Rev. Mod. Phys., vol. 87, pp. 897-923, Aug 2015. 
[29] C. W. Groth, M. Wimmer, A. R. Akhmerov, and X. Waintal, "Kwant: A software package for quantum transport," New Journal of Physics, vol. 16, pp. 1-19, 2014.

[30] E. N. Lorenz, "Deterministic Nonperiodic Flow," 1963.

[31] E. Benincà, B. Ballantine, S. P. Ellner, and J. Huisman, "Species fluctuations sustained by a cyclic succession at the edge of chaos," Proceedings of the National Academy of Sciences, vol. 112, no. 20, pp. 6389-6394, 2015.

[32] P. Bittihn, S. Berg, U. Parlitz, and S. Luther, "Emergent dynamics of spatio-temporal chaos in a heterogeneous excitable medium," Chaos: An Interdisciplinary Journal of Nonlinear Science, vol. 27, p. 093931, Sept. 2017.

[33] D. Gonze, K. Z. Coyte, L. Lahti, and K. Faust, "Microbial communities as dynamical systems," Current Opinion in Microbiology, vol. 44, pp. 41-49, Aug. 2018.

[34] Y. Jung, E. Barkai, and R. J. Silbey, "Lineshape theory and photon counting statistics for blinking quantum dots: a lévy walk process," Chemical Physics, vol. 284, pp. 181-194, Nov. 2002.

[35] D. A. Kessler and E. Barkai, "Infinite covariant density for diffusion in logarithmic potentials and optical lattices," Phys. Rev. Lett., vol. 105, p. 120602, Sep 2010.

[36] R. Fleischmann, T. Geisel, and R. Ketzmerick, "Magnetoresistance due to chaos and nonlinear resonances in lateral surface superlattices," Physical Review Letters, vol. 68, no. 9, pp. 1367-1370, 1992.

[37] L. A. Bunimovich and L. V. Vela-Arevalo, "Some new surprises in chaos," Chaos, vol. 25, p. 097614, June 2015.

[38] G. Datseris, T. Geisel, and R. Fleischmann, "Robustness of ballistic transport in antidot superlattices," New Journal of Physics, vol. 21, p. 043051, Apr. 2019.

[39] D. Weiss, M. L. Roukes, A. Menschig, P. Grambow, K. von Klitzing, and G. Weimann, "Electron pinball and commensurate orbits in a periodic array of scatterers," Phys. Rev. Lett., vol. 66, pp. 2790-2793, May 1991.

[40] N. I. Chernov, G. L. Eyink, J. L. Lebowitz, and Y. G. Sinai, "Steady-state electrical conduction in the periodic Lorentz gas," Commun.Math. Phys., vol. 154, pp. 569-601, June 1993.

[41] R. Yagi, R. Sakakibara, R. Ebisuoka, J. Onishi, K. Watanabe, T. Taniguchi, and Y. Iye, "Ballistic transport in graphene antidot lattices," Physical Review B, 2015.

[42] H. Maier, J. Ziegler, R. Fischer, D. Kozlov, Z. D. Kvon, N. Mikhailov, S. A. Dvoretsky, and D. Weiss, "Ballistic geometric resistance resonances in a single surface of a topological insulator," Nature Communications, vol. 8, no. 1, pp. 1-6, 2017.

[43] S. Chen, Z. Han, M. M. Elahi, K. M. M. Habib, L. Wang, B. Wen, Y. Gao, T. Taniguchi, K. Watanabe, J. Hone, A. W. Ghosh, and C. R. Dean, "Electron optics with p-n junctions in ballistic graphene," Science, vol. 353, no. 6307, 2016.

[44] J. P. Bird, ed., Electron Transport in Quantum Dots. Springer US, 2003.

[45] C. Beenakker, "Andreev billiards," in Quantum Dots: a Doorway to Nanoscale Physics, pp. 131-174, Springer Berlin Heidelberg, mar 2005. 
[46] L. A. Ponomarenko, F. Schedin, M. I. Katsnelson, R. Yang, E. W. Hill, K. S. Novoselov, and A. K. Geim, "Chaotic dirac billiard in graphene quantum dots," Science, vol. 320, pp. 356-358, apr 2008.

[47] H.-J. Stöckmann and J. Stein, "“Quantum” chaos in billiards studied by microwave absorption," Phys. Rev. Lett., vol. 64, pp. 2215-2218, May 1990.

[48] A. H. Barnett and T. Betcke, "Quantum mushroom billiards," Chaos Interdiscip. J. Nonlinear Sci., vol. 17, p. 043125, Dec. 2007.

[49] A. D. Stone, "Nonlinear dynamics: Chaotic billiard lasers," Nature, vol. 465, pp. 696-697, June 2010.

[50] C. Gmachl, F. Capasso, E. E. Narimanov, J. U. Nöckel, A. D. Stone, J. Faist, D. L. Sivco, and A. Y. Cho, "High-Power Directional Emission from Microlasers with Chaotic Resonators," Science, vol. 280, pp. 1556-1564, June 1998.

[51] F. Miao, S. Wijeratne, Y. Zhang, U. C. Coskun, W. Bao, and C. N. Lau, "Phase-coherent transport in graphene quantum billiards," Science, vol. 317, pp. 1530-1533, Sept. 2007.

[52] P. Yu, Z.-Y. Li, H.-Y. Xu, L. Huang, B. Dietz, C. Grebogi, and Y.-C. Lai, "Gaussian orthogonal ensemble statistics in graphene billiards with the shape of classically integrable billiards," Phys. Rev. E, vol. 94, p. 062214, Dec 2016.

[53] M. Deryabin and L. Pustyl'nikov, "Generalized Relativistic Billiards," Regular and Chaotic Dynamics, vol. 8, no. 3, p. 283, 2003.

[54] A. M. Lyapunov, "The general problem of the stability of motion," International Journal of Control, vol. 55, no. 3, pp. 531-534, 1992.

[55] G. Benettin, L. Galgani, A. Giorgilli, and J.-M. Strelcyn, "Lyapunov characteristic exponents for smooth dynamical systems and for hamiltonian systems. a method for computing all of them. part 2: Numerical application," Meccanica, vol. 15, pp. 21-30, Mar. 1980.

[56] K. Geist, U. Parlitz, and W. Lauterborn, "Comparison of Different Methods for Computing Lyapunov Exponents," Progress of Theoretical Physics, vol. 83, no. 5, pp. 875-893, 1990.

[57] U. Parlitz, "Estimating lyapunov exponents from time series," in Chaos Detection and Predictability, pp. 1-34, Springer Berlin Heidelberg, 2016.

[58] S. H. Strogatz, Nonlinear Dynamics and Chaos: With Applications to Physics, Biology, Chemistry and Engineering. Westview Press, 2000.

[59] B. R. Hunt and E. Ott, "Defining chaos," Chaos, vol. 25, no. 9, 2015.

[60] M. Tabor, Chaos and Integrability in Nonlinear Dynamics. Wiley, 1989.

[61] H. G. Schuster and W. Just, Deterministic Chaos. Wiley, Jan. 2005.

[62] A. Sandner, T. Preis, C. Schell, P. Giudici, K. Watanabe, T. Taniguchi, D. Weiss, and J. Eroms, "Ballistic Transport in Graphene Antidot Lattices," Nano Letters, vol. 15, pp. 8402-8406, dec 2015.

[63] S. Bauch, a. Błedowski, L. Sirko, P. Koch, and R. Blümel, "Signature of non-Newtonian orbits in ray-splitting cavities," Physical Review E, vol. 57, no. 1, pp. 304-315, 1998.

[64] R. Schäfer, U. Kuhl, M. Barth, and H. J. Stöckmann, "Spectra and wavefunctions in a ray-splitting Sinai microwave billiard and their semiclassical interpretation," Foundations of Physics, vol. 31, no. 3, pp. 475-487, 2001. 
[65] R. Blümel, P. M. Koch, and L. Sirko, "Ray-Splitting Billiards," Foundations of Physics, vol. 31, no. 2, pp. 269-281, 2001.

[66] R. Landauer, "Spatial variation of currents and fields due to localized scatterers in metallic conduction," IBM Journal of Research and Development, vol. 1, pp. 223-231, July 1957.

[67] M. Razavy, Quantum Theory of Tunneling. World Scientific, Jan. 2003.

[68] D. J. Griffiths and D. F. Schroeter, Introduction to Quantum Mechanics. Cambridge University Press, Aug. 2018.

[69] P. E. Allain and J. N. Fuchs, "Klein tunneling in graphene: Optics with massless electrons," European Physical Journal B, vol. 83, no. 3, pp. 301-317, 2011.

[70] V. V. Cheianov and V. I. Fal'ko, "Selective transmission of Dirac electrons and ballistic magnetoresistance of p-n junctions in graphene," Physical Review B, vol. 74, p. 041403, jul 2006.

[71] M. I. Katsnelson, K. S. Novoselov, and a. K. Geim, "Chiral tunneling and the Klein paradox in graphene," Nature physics, vol. 2, no. September, p. 15, 2006.

[72] O. Klein, "Die Reflexion von Elektronen an einem Potentialsprung nach der relativistischen Dynamik von Dirac," Zeitschrift für Physik, vol. 53, pp. 157-165, mar 1929.

[73] T. Curtright, D. Fairlie, and C. Zachos, A concise treatise on quantum mechanics in phase space. 2013.

[74] N. Bohr, "Uber die serienspektra der elemente," Zeitschrift fur Physik, vol. 2, pp. 423-469, Oct. 1920.

[75] P. Ehrenfest, "Bemerkung uber die angenaherte gultigkeit der klassischen mechanik innerhalb der quantenmechanik," Zeitschrift fur Physik, vol. 45, pp. 455-457, July 1927.

[76] E. Wigner, "On the Quantum Correction For Thermodynamic Equilibrium," Physical Review, vol. 40, pp. 749-759, jun 1932.

[77] K. Husimi, "Some Formal Properties of the Density Matrix," Proceedings of the PhysicoMathematical Society of Japan. 3rd Series, vol. 22, no. 4, pp. 264-314, 1940.

[78] K. Takahashi, "Wigner and husimi functions in quantum mechanics," Journal of the Physical Society of Japan, vol. 55, pp. 762-779, mar 1986.

[79] W. P. Schleich, Quantum Optics in Phase Space. Wiley, Feb. 2001.

[80] H. Moya-Cessa, J. R. Moya-Cessa, and L. R. Berriel-Valdos, "Introduction to quantum optics: quasiprobability distribution functions," Introduction to quantum optics, no. 1, p. $31,2008$.

[81] A. L. Virovlyansky, D. V. Makarov, and S. V. Prants, "Ray and wave chaos in underwater acoustic waveguides," p. 30, 2012.

[82] S. Nonnenmacher and A. Voros, "Chaotic Eigenfunctions in Phase Space," Journal of Statistical Physics, vol. 92, pp. 431-518, Aug. 1998.

[83] A. Bäcker, "Numerical Aspects of Eigenvalue and Eigenfunction Computations for Chaotic Quantum Systems," in The Mathematical Aspects of Quantum Maps (M. D. Esposti and S. Graffi, eds.), (Berlin, Heidelberg), pp. 91-144, Springer Berlin Heidelberg, 2003. 
[84] A. Bäcker, S. Fürstberger, and R. Schubert, "Poincar \'e Husimi representation of eigenstates in quantum billiards," Physical Review E, vol. 70, p. 036204, Sept. 2004.

[85] A. Bäcker, R. Ketzmerick, and A. G. Monastra, "Flooding of Chaotic Eigenstates into Regular Phase Space Islands," Physical Review Letters, vol. 94, p. 054102, Feb. 2005.

[86] F. Toscano, A. Kenfack, A. R. Carvalho, J. M. Rost, and A. M. Ozorio de Almeida, "Husimi-Wigner representation of chaotic eigenstates," Proceedings of the Royal Society A: Mathematical, Physical and Engineering Sciences, vol. 464, pp. 1503-1524, June 2008.

[87] H. Schanz, T. Dittrich, and R. Ketzmerick, "Directed chaotic transport in Hamiltonian ratchets," Physical Review E, vol. 71, p. 026228, Feb. 2005.

[88] M. Hentschel, H. Schomerus, and R. Schubert, "Husimi functions at dielectric interfaces: Inside-outside duality for optical systems and beyond," EPL (Europhysics Letters), vol. 62, p. 636, June 2003.

[89] J. Wiersig and M. Hentschel, "Combining Directional Light Output and Ultralow Loss in Deformed Microdisks," Physical Review Letters, vol. 100, p. 033901, Jan. 2008.

[90] A. Bäcker, R. Ketzmerick, S. Löck, J. Wiersig, and M. Hentschel, "Quality factors and dynamical tunneling in annular microcavities," Physical Review A, vol. 79, p. 063804, June 2009.

[91] K. W. Mahmud, H. Perry, and W. P. Reinhardt, "Quantum phase-space picture of BoseEinstein condensates in a double well," Physical Review A, vol. 71, p. 023615, Feb. 2005.

[92] D. J. Mason, M. F. Borunda, and E. J. Heller, "Semiclassical deconstruction of quantum states in graphene," Physical Review B - Condensed Matter and Materials Physics, vol. 88, no. 16, pp. 1-9, 2013.

[93] D. Mason, M. Borunda, and E. Heller, "Quantum flux and reverse engineering of quantum wave functions," EPL (Europhysics Letters), vol. 60005, no. 1, pp. 1-5, 2013.

[94] D. J. Mason, M. F. Borunda, and E. J. Heller, "Revealing the flux: Using processed Husimi maps to visualize dynamics of bound systems and mesoscopic transport," Physical Review $B$, vol. 91, no. 16, p. 165405, 2015.

[95] K. v. Klitzing, G. Dorda, and M. Pepper, "New Method for High-Accuracy Determination of the Fine-Structure Constant Based on Quantized Hall Resistance," Physical Review Letters, vol. 45, pp. 494-497, aug 1980.

[96] K. S. Novoselov, Z. Jiang, Y. Zhang, S. V. Morozov, H. L. Stormer, U. Zeitler, J. C. Maan, G. S. Boebinger, P. Kim, and A. K. Geim, "Room-Temperature Quantum Hall Effect in Graphene," Science, vol. 315, pp. 1379-1379, mar 2007.

[97] D. Weiss, M. L. Roukes, a. Menschig, P. Grambow, K. Von Klitzing, and G. Weimann, "Electron pinball and commensurate orbits in a periodic array of scatterers," Physical Review Letters, vol. 66, no. 21, pp. 2790-2793, 1991.

[98] K. Ensslin and P. M. Petroff, "Magnetotransport through an antidot lattice in GaAsAlxGa1-xAs heterostructures," Phys. Rev. B, vol. 41, pp. 12307-12310, June 1990.

[99] A. Lorke, J. P. Kotthaus, and K. Ploog, "Magnetotransport in two-dimensional lateral superlattices," Phys. Rev. B, vol. 44, no. 7, pp. 3447-3450, 1991. 
[100] Z. L. Xiao, C. Y. Han, U. Welp, H. H. Wang, V. K. Vlasko-Vlasov, W. K. Kwok, D. J. Miller, J. M. Hiller, R. E. Cook, G. A. Willing, and G. W. Crabtree, "Nickel antidot arrays on anodic alumina substrates," Applied Physics Letters, vol. 81, pp. 2869-2871, oct 2002.

[101] R. Wördenweber, P. Dymashevski, and V. R. Misko, "Guidance of vortices and the vortex ratchet effect in high-tc superconducting thin films obtained by arrangement of antidots," Physical Review B, vol. 69, p. 184504, may 2004.

[102] S. Neusser, B. Botters, and D. Grundler, "Localization, confinement, and field-controlled propagation of spin waves in ni80fe20 antidot lattices," Physical Review B, vol. 78, p. 054406, aug 2008.

[103] R. Fleischmann, T. Geisel, C. Holzknecht, and R. Ketzmerick, "Nonlinear dynamics of composite fermions in nanostructures," Europhysics Letters (EPL), vol. 36, p. 167, 1996.

[104] D. Weiss, G. Lütjereng, and K. Richter, "Chaotic electron motion in macroscopic and mesoscopic antidot lattices," Chaos, Solitons \& Fractals, vol. 8, no. 7-8, pp. 1337-1357, 1997.

[105] J. a. Fürst, J. G. Pedersen, C. Flindt, N. A. Mortensen, M. Brandbyge, T. G. Pedersen, and A.-P. Jauho, "Electronic properties of graphene antidot lattices," New Journal of Physics, vol. 11, 2009.

[106] R. Schuster, G. Ernst, K. Ensslin, M. Entin, M. Holland, G. B??hm, and W. Klein, "Experimental characterization of electron trajectories in antidot lattices," Physical Review B, vol. 50, no. 11, pp. 8090-8093, 1994.

[107] H. Silberbauer and U. Rossler, "Quantum study of magnetotransport in antidot superlattices," Phys. Rev. B, vol. 50, no. 16, 1994.

[108] S. Ishizaka and T. Ando, "Detailed analysis of the commensurability peak in antidot arrays with various periods," Physical Review B, vol. 55, no. 24, pp. 16331-16338, 1997.

[109] T. Ando and S. Uryu, "Chaotic transport in antidot lattices," Journal of Electronic Materials, vol. 29, no. 5, pp. 557-564, 2000.

[110] Y. G. Sinai, "Dynamical systems with elastic reflections," Russ. Math. Surv., vol. 25, no. 2, p. 137,1970 .

[111] M. Fließer, G. J. O. Schmidt, and H. Spohn, "Magnetotransport of the Sinai billiard," Phys. Rev. E, vol. 53, pp. 5690-5697, June 1996.

[112] R. Kubo, "Statistical-Mechanical Theory of Irreversible Processes. I.," J. Phys. Soc. Jpn., vol. 12 , no. 6 , pp. 570-586, 1957.

[113] George Datseris, "Master thesis, National and Kapodistrian University of Athens," 2016. [The thesis is unavailable online, but can be shared on request].

[114] M. Kac, "On the notion of recurrence in discrete stochastic processes," Bull. Amer. Math. Soc, vol. 20, pp. 376-379, 1947.

[115] R. S. MacKay, "Transport in 3D volume-preserving flows," Journal of Nonlinear Science, vol. 4, no. 1, pp. 329-354, 1994.

[116] J. D. Meiss, "Average exit time for volume-preserving maps," Chaos, vol. 7, no. 1, pp. 139$147,1997$. 
[117] E. G. Altmann and T. Tel, "Poincaré recurrences from the perspective of transient chaos," Phys. Rev. Lett., vol. 100, no. 17, pp. 1-4, 2008.

[118] L. A. Bunimovich and L. V. Vela-Arevalo, "Some new surprises in chaos (billiards)," Chaos, vol. 25, no. 9, 2015.

[119] L. A. Bunimovich, "On ergodic properties of certain billiards," Funct Anal Its Appl, vol. 8, pp. 254-255, July 1974.

[120] L. A. Bunimovich, "On the ergodic properties of nowhere dispersing billiards," Commun.Math. Phys., vol. 65, pp. 295-312, Oct. 1979.

[121] S. Koyanagi, T. Nakano, and T. Kawabe, "Application of Hamiltonian of ray motion to room acoustics," J. Acoust. Soc. Am., vol. 124, pp. 719-722, Aug. 2008.

[122] G. Datseris, T. Geisel, and R. Fleischmann, "Robustness of ballistic transport in antidot superlattices," New Journal of Physics, vol. 21, p. 043051, apr 2019.

[123] G. Datseris, "DynamicalBilliards.jl: An easy-to-use, modular and extendable julia package for dynamical billiard systems in two dimensions.," The Journal of Open Source Software, vol. 2, p. 458, nov 2017 .

[124] L. A. Bunimovich, "Mushrooms and other billiards with divided phase space," Chaos Interdiscip. J. Nonlinear Sci., vol. 11, pp. 802-808, Dec. 2001.

[125] Z. Vörös, T. Tasnádi, J. Cserti, and P. Pollner, "Tunable Lyapunov exponent in inverse magnetic billiards," Phys. Rev. E, vol. 67, June 2003.

[126] C. H. Skokos, Chaos Detection and Predictability, vol. 915 of Lecture Notes in Physics. Berlin, Heidelberg: Springer Berlin Heidelberg, 2016.

[127] M. O. Goerbig, "Electronic Properties of Graphene in a Strong Magnetic Field," Rev. Mod. Phys., vol. 83, no. October, p. 1193, 2011.

[128] S. Das Sarma, S. Adam, E. H. Hwang, and E. Rossi, "Electronic transport in twodimensional graphene," Reviews of Modern Physics, vol. 83, no. 2, pp. 407-470, 2011.

[129] M. I. Katsnelson, Graphene: Carbon in Two Dimensions. Cambridge University Press, 2012.

[130] V. P. Gusynin, S. G. Sharapov, and J. P. Carbotte, "AC conductivity of graphene: from tight-binding model to $2+1$-dimensional quantum electrodynamics," Int. J. Mod. Phys., vol. B21, no. 27, pp. 4611-4658, 2007.

[131] T. Ando, T. Nakanishi, and R. Saito, "Berry's Phase and Absence of Back Scattering in Carbon Nanotubes," Journal of the Physical Society of Japan, vol. 67, no. 8, pp. 2857-2862, 1998.

[132] B. Huard, J. A. Sulpizio, N. Stander, K. Todd, B. Yang, and D. Goldhaber-Gordon, "Transport measurements across a tunable potential barrier in graphene," Physical Review Letters, vol. 98, no. 23, pp. 8-11, 2007.

[133] L. Brey and H. a. Fertig, "Edge states and the quantized Hall effect in graphene," Physical Review B - Condensed Matter and Materials Physics, vol. 73, no. 19, pp. 1-5, 2006.

[134] L. Brey and H. a. Fertig, "Electronic states of graphene nanoribbons studied with the Dirac equation," Physical Review B - Condensed Matter and Materials Physics, vol. 73, no. 23, pp. 2-6, 2006. 
[135] M. Ezawa, "Peculiar width dependence of the electronic properties of carbon nanoribbons," Phys. Rev. B, vol. 73, p. 045432, Jan 2006.

[136] A. Sandner, High-mobility graphene in 2D periodic potentials. PhD thesis, University of Regensburg, 2017.

[137] K. Takahashi, "Wigner and Husimi functions in Quantum Mechanics," Journal of Physical Society of Japan, vol. 55, no. 3, 1985.

[138] R. Fleischmann, T. Geisel, and R. Ketzmerick, "Quenched and negative hall effect in periodic media: application to antidot superlattices," Europhys. Lett., vol. 25, no. January, pp. 219-224, 1994.

[139] N. Chernov, "Entropy, Lyapunov exponents, and mean free path for billiards," J. Stat. Phys., vol. 88, pp. 1-29, July 1997.

[140] J. Bezanson, A. Edelman, S. Karpinski, and V. B. Shah, "Julia: A fresh approach to numerical computing," SIAM Review, vol. 59, no. 1, pp. 65-98, 2017.

[141] D. Tapias, D. P. Sanders, and E. G. Altmann, "Monte carlo sampling in diffusive dynamical systems," Chaos: An Interdisciplinary Journal of Nonlinear Science, vol. 28, p. 053113, May 2018.

[142] J. Solanpää, P. Luukko, and E. Räsänen, "Bill2d — a software package for classical twodimensional hamiltonian systems," Computer Physics Communications, vol. 199, pp. 133138, Feb. 2016.

[143] C. Dellago, H. A. Posch, and W. G. Hoover, "Lyapunov instability in a system of hard disks in equilibrium and nonequilibrium steady states," Phys. Rev. E, vol. 53, pp. 1485-1501, Feb. 1996.

[144] A. V. Shytov, N. Gu, and L. S. Levitov, "Transport in Graphene p-n Junctions in Magnetic Field," arXiv preprint arXiv:0708.3081, no. 2, pp. 1-4, 2007.

[145] A. V. Shytov, M. S. Rudner, and L. S. Levitov, "Klein backscattering and Fabry-P??rot interference in graphene heterojunctions," Physical Review Letters, vol. 101, no. 15, pp. 1013, 2008.

[146] N. Stander, B. Huard, and D. Goldhaber-Gordon, "Evidence for Klein tunneling in graphene p-n junctions," Physical Review Letters, vol. 102, no. 2, pp. 1-4, 2009.

[147] A. F. Young and P. Kim, "Quantum interference and Klein tunneling in graphene heterojunctions," Nature Physics, vol. 5, no. 3, p. 13, 2008.

[148] E. Brown, "Bloch electrons in a uniform magnetic field," Physical Review, vol. 133, no. 4A, 1964.

[149] J. Zak, "Magnetic Translation Group," Physical Review, vol. 134, pp. A1602-A1606, jun 1964.

[150] M. H. Liu, C. Gorini, and K. Richter, "Creating and Steering Highly Directional Electron Beams in Graphene," Physical Review Letters, vol. 118, no. 6, pp. 1-6, 2017. 


\section{George Datseris}

Curriculum Vitae

\section{Academic Background}

10/2019 - Postdoctoral fellow, Max Planck Institute for Dynamics and Self-

12/2019 organization, Department of Living Matter Physics (dir. Ramin Golestanian), Göttingen, Germany.

04/2016 - PhD in physics: Ballistic electron transport in graphene nanodevices

09/2019 and billiards (awareded "summa cum laude"), Max Planck Institute for Dynamics and Self-organization, Department of Nonlinear Dynamics (dir. Theo Geisel), Göttingen, Germany.

PhD supervisors: Prof. Theo Geisel, Dr. Ragnar Fleischmann

10/2015 - Research Assistant, Max Planck Institute for Dynamics and Self-

04/2016 Organization, Department of Nonlinear Dynamics (dir. Theo Geisel).

6-month funded period that constituted my MSc Thesis.

2014 - 2016 Master's Degree in the specialty of "Physics of Materials" (120 ECTS), National and Kapodistrian University of Athens, Faculty of Physics, Athens, Grade 9.28/10.0.

Thesis: Quasi-classical Magneto-transport in Graphene Antidot Super-lattices (supervised by Prof. Theo Geisel \& Prof. Georgios Triberis)

2009 - 2014 Bachelor in Physics (240 ECTS), Majored in Solid State Physics, National and Kapodistrian University of Athens, Faculty of Physics, Athens, Grade 8.06/10.0.

Thesis: Auto- and Cross- Correlations in the Standard Map: Structure and Origin (supervised by Prof. Fotios Diakonos)

\section{Fellowships \& Awards}

2018 DSWeb 2018 Dynamical System Software Competition by SIAM.

First place winner using the software DynamicalSystems.jl as a submission. For more details please see the official statement.

2018 IMPRS Travel Grants.

For travelling to London, U.K. and participating in the conference JuliaCon 2018 as well as travelling to Potsdam, Germany and participating in the conference deRSE19.

2016 - 2019 International Max Planck Research School Scholarship.

This 3-years scholarship covers a full PhD project and was awarded to me on July/2016 for my $\mathrm{PhD}$ project. 


\section{Publications}

2019 Phase space analysis of quantum transport in graphene, G. Datseris \& R. Fleischmann, submitted (https://arxiv.org/abs/1905.06637).

2019 Estimating Lyapunov exponents in billiards, G. Datseris, L. Hupe \& R. Fleischmann, Chaos (doi.org/10.1063/1.5099446).

2019 Does it Swing? Microtiming Deviations and Swing Feeling in Jazz, G. Datseris, A. Zieries, T. Albrecht, V. Priesemann, Y. Hagmayer \& T. Geisel, submitted to Scientific Reports (arxiv.org/abs/1904.03442).

2019 Predicting Spatio-Temporal Time Series Using Dimension Reduced Local States, J. Isensee, G. Datseris \& U. Parlitz, accepted in J. Nonlin. Sci..

2019 Robustness of ballistic transport in antidot superlattices, G. Datseris, T. Geisel \& R. Fleischmann, New J. Phys. (doi.org/10.1088/1367-2630/ab19cc).

2019 MIDI.jl: Simple and intuitive handling of MIDI data, G. Datseris \& J. Hobson, Journal of Open Source Software, 4(35), 1166, https://doi.org/10.21105/joss.01166.

2018 DynamicalSystems.jl: A Julia software library for chaos and nonlinear dynamics, G. Datseris, Journal of Open Source Software, 3(23), 598, https://doi.org/10.21105/joss.00598.

2017 DynamicalBilliards.jl: An easy-to-use, modular and extendable Julia package for Dynamical Billiard systems in two dimensions, G. Datseris, Journal of Open Source Software, 2(19), 458, doi:10.21105/joss.00458.

2015 Effective intermittency and cross correlations in the standard map, G. Datseris, P. Schmelcher \& F. Diakonos, Phys. Rev. E 92, 012914 (2015).

\section{Developed and Published Software}

○ InteractiveChaos.jl - Interactive applications for the exploration of chaos and nonlinear dynamics.

- DynamicalSystems.jl - Award winning software library for exploring nonlinear dynamics and chaos.

○ DynamicalBilliards.jl - An easy-to-use, modular, performant and extendable Julia package for dynamical billiards in two dimensions.

- TimeseriesPrediction.jl - Prediction of timeseries using methods of nonlinear dynamics and timeseries analysis.

- DrWatson - A software package that helps scientists deal with their scientific projects.

○ MusicManipulations.jl - Manipulate music data, humanize, quantize and enhance performances with Julia.

○ MIDI.jl - A Julia library for handling MIDI files.

- MotifSequenceGenerator.jl - Generate random sequences of motifs, under the constrain that the sequence has some given total length.

(8) +49-(0)160-98278727 • 凶 datseris.george@gmail.com

四 datseris.github.io 


\section{Additional Qualifications}

Programming Python, Julia, ATEX, Microsoft Office, Cubase, Mathematica, Adobe Photoshop, Adobe Illustrator, C.

Soft Skills Attended courses on networking, negotiation, conflict management, grant writing, career development.

Projects Attended a 7-weeks course on project planning, integration, management and productivity.

Professional Graduated from Philippos Nakas school of music in association with Berklee

Drummer college of music with diploma grade: "Very Good". During my drumming career I have been leading numerous groups of amateur and professional musicians, obtaining skills in leadership and teamwork. The diploma in modern drumset and theory of jazz music was awarded to me on July 2015.

\section{Invited Talks}

11/2019 Phase space analysis of quantum transport in graphene, Technical University Vienna, invited by Stefan Rotter.

09/2019 Music timeseries analysis: universal structure and its impact on the listening experience, University of Nottingham, invited by Philip Moriarty.

08/2019 Fresh approach to dynamical systems software, $8^{\text {th }}$ Recurrence Plot Symposium - Zhenjiang, China, invited by Norbert Marwan.

07/2019 Software to make your scientific life easier, New trends in biomedical imaging and data analysis (Conference), invited by Ulrich Parlitz.

05/2019 Music timeseries analysis: universal structure and its impact on the listening experience, Max Planck Institute for the Physics of Complex Systems, invited by Holger Kantz.

04/2019 Spatiotemporal Timeseries prediction using locally reconstructed states, Potsdam Institute for Climate Impact Research, invited by Norbert Marwan.

07/2018 Fresh approach to dynamical systems software, TU Munich, invited by Oliver Junge.

04/2018 Nonlinear Resonances and phase-space volume conservation lead to robust ballistic transport in antidot superlattices, Uni. Regensburg, invited by Jonathan Eroms.

\section{Supervised Theses}

2018 Statistical properties of musical time series, L. Jahn, Bachelor thesis co-supervised with T. Geisel.

2018 Observing and predicting complex dynamics using local modelling, J. Isensee, Bachelor thesis co-supervised with U. Parlitz.

2018 Lyapunov exponents vs. phase space restrictions in dynamical billiards, L. Hupe, Bachelor thesis co-supervised with R. Fleischmann.

$$
\begin{gathered}
\text { (8) +49-(0)160-98278727 • } \bullet \text { datseris.george@gmail.com } \\
\text { 四 datseris.github.io }
\end{gathered}
$$




\section{Teaching}

\section{Lectures}

2017 Introduction to the Physics of Complex Systems, University of Göttingen.

Tutoring for the course (total amount of around 48 hours) with lecturers R. Fleischmann, U. Parlit and A. Gholami.

2016 Introduction to the Physics of Complex Systems, University of Göttingen.

Tutoring for the course (total amount of around 48 hours) with lecturers R. Fleischmann, U. Parlit and K. Alim.

04/2014 - Chaos in 1D and 2D Maps, University of Athens.

06/2014 Total of 9 hours lecture for the course called "Non-linear dynamical systems". The units I taught undergraduate students were 1D Chaotic Maps, 2D Hamiltonian Maps, Lyapunov exponents and Invariant measures.

Workshops, Videos, Other

2017-2019 Software video tutorials, Online.

Multiple videos uploaded (or livestreamed) on YouTube explaining the use of software described in the Software section of the CV.

2017 Jumping into Julia, Max Planck Institute for Dynamics and SelfOrganization.

Single day workshop about the programming language Julia and how can one start using it in scientific work.

\section{Event Organizing}

10/2019 Hacktoberfest meetup at the Max Planck Institute for Dynamics and SelfOrganization

2018 - 2019 Göttingen GGNB Debate club.

2018 Bi-annual retreat of the PhD school for the Physics of Biological and Complex Systems.

\section{Languages}

Mothertongue Greek

Expert English

Intermediate Spanish

Proficiency degree from the University of Michigan

B2 National degree 